\title{
Neutron Capture and Transmission Measurements and Resonance Parameter Analysis of Gadolinium
}

G Leinweber, DP Barry, MJ Trbovich, JA Burke, NJ Drindak, HD Knox, RV Ballad, RC Block, Y Danon, LI Severnyak

This report was prepared as an account of work sponsored by the United States Government. Neither the United States, nor the United States Department of Energy, nor any of their employees, nor any of their contractors, subcontractors, or their employees, makes any warranty, express or implied, or assumes any legal liability or responsibility for the accuracy, completeness or usefulness of any information, apparatus, product or process disclosed, or represents that its use would not infringe privately owned rights. 


\title{
Neutron Capture and Transmission Measurements and Resonance Parameter Analysis of Gadolinium
}

\author{
G Leinweber, DP Barry, MJ Trbovich, JA Burke, NJ Drindak, HD Knox, and RV Ballad \\ Lockheed Martin Corporation, P.O. Box 1072, Schenectady, New York 12301-1072 \\ RC Block, Y Danon, LI Severnyak \\ Rensselaer Polytechnic Institute, Department of Mechanical, Aerospace, and Nuclear \\ Engineering, Troy, New York 12180-3590
}

July 2005

\begin{abstract}
Neutron capture and transmission measurements were performed by the timeof-flight technique at the Rensselaer Polytechnic Institute (RPI) linac facility using metallic and liquid Gd samples. The liquid samples were isotopically-enriched in either ${ }^{155} \mathrm{Gd}$ or ${ }^{157} \mathrm{Gd}$. The capture measurements were made at the $25-m$ flight station with a multiplicity-type capture detector, and the transmission measurements were performed at 15- and 25-m flight stations with ${ }^{6} \mathrm{Li}$ glass scintillation detectors. The multilevel R-matrix Bayesian code SAMMY was used to extract resonance parameters.
\end{abstract}

Among the significant findings are the following. The neutron width of the largest resonance in $G d$, at $0.032 \mathrm{eV}$ in ${ }^{157} \mathrm{Gd}$, has been measured to be $(9 \pm 1) \%$ smaller than that given in ENDF/B-VI updated through release 8 . The thermal $(2200 \mathrm{~m} / \mathrm{s})$ capture cross section of ${ }^{157} \mathrm{Gd}$.has been measured to be $11 \%$ smaller than that calculated from $E N D F$. The other major thermal resonance, at $0.025 \mathrm{eV}$ in ${ }^{155} \mathrm{Gd}$, did not display a significant deviation from the thermal capture cross section given by ENDF.

In the epithermal region, 1-300 eV, the analysis provided here represents the most extensive to date. Twenty eight new resonances are proposed and other resonances previously identified in the literature have been revisited. The assignment of resonances within regions of complicated structure incorporated the observations of other researchers, particularly on the six occasions where ENDF resonances are recommended to be removed. The poor match of the ENDF parameters to the current data is significant, and substantial improvement to the understanding of gadolinium cross sections is presented, particularly above $180 \mathrm{eV}$ where the ENDF resolved region for ${ }^{155} \mathrm{Gd}$ ends.

In the epithermal energy range, natural metal samples were measured in capture and transmission. The data were analyzed with the Bayesian code SAMMY. Resonance parameters and resonance integrals have been calculated. The historical record was reviewed during the analysis and judgments have been made regarding the number and location of new epithermal resonances. The data ciearly show that some resonances are needed to supplement those given in ENDF. The isotope and spin of the new resonances could not be determined and their neutron widths were reported as $2 a g \Gamma_{n}$. 


\section{Introduction}

For many years, the accuracy of measured cross sections exceeded that required by the computer codes used for nuclear reactor calculations. With the increased use of Monte Carlo methods utilizing increased computer memory for more detailed models and greater speed to permit more histories, this situation changed. The accuracy of the crosssection data is now the limiting factor in many of these calculations.

The purpose of the present work is to measure the neutron cross sections of gadolinium accurately. Gd has the highest thermal cross section of any natural element. Two large resonances exist slightly above thermal energy. The resonances are in ${ }^{155} \mathrm{Gd}$ and ${ }^{157} \mathrm{Gd}$. The resonances are not resolved despite the use of separated isotopes. Isotopically-enriched ${ }^{155} \mathrm{Gd}$ and ${ }^{157} \mathrm{Gd}$ samples were prepared as liquid solutions with heavy water to produce uniform, thin samples. These thin samples were used in conjunction with elemental, natural metal samples.

To achieve high-accuracy measured data, the KAPL/RPI (Rensselaer Polytechnic Institute) Cross-Section Group has been actively involved in upgrading the experimental equipment used in the measurement program at the RPI linear accelerator (linac) facility. A high-efficiency, 16-segment, multiplicity-type gamma detector has been developed for use in neutron capture measurements. ${ }^{1,2}$ An improved time-of-flight (TOF) analyzer, which is interfaced directly to a data-acquisition computer, has been designed and constructed to achieve high accuracy and handle high count rate measurements. ${ }^{1,3}$ Improved linac targets have been developed to provide enhanced neutron production at energies of interest. ${ }^{4,5,6}$ Special-purpose data reduction software has been written to manipulate the data arrays, and the state-of-the-art SAMMY version M6 Bayesian analysis program ${ }^{7}$ has been obtained from Oak Ridge National Laboratory for neutron resonance parameter determination. The neutron resonance parameters of interest are resonance energy, $E_{0}$, neutron width, $\Gamma_{\mathrm{n}}$, and radiation width, $\Gamma_{\gamma}$.

\section{Historical Review}

A review of the prior measurements of $\mathrm{Gd}$ shows that the resonance parameters for the low energy doublet (at $\approx 0.03 \mathrm{eV}$ ) in ENDF/B-VI ${ }^{8}$ updated through release 8 are nearly identical to those of Moller et al. ${ }^{9}$ The neutron width of the low energy ${ }^{155} \mathrm{Gd}$ resonance and the radiation widths of both low energy resonances come directly from the paper of Moller et al. ${ }^{9}$, while the ENDF value for the neutron width of the low energy ${ }^{157} \mathrm{Gd}$ resonance is within $0.4 \%$ of the value given by Moller et al. ${ }^{9}$

In the region from 1.0 to $300.0 \mathrm{eV}$, most of the resonances occur in ${ }^{155} \mathrm{Gd}$ and ${ }^{157} \mathrm{Gd}$. In these two isotopes, ENDF resonance parameters are based on a few experiments, particularly Mughabghab and Chrien ${ }^{10}$, Simpson ${ }^{11}$, and Fricke et al. ${ }^{12}$ The other high-abundance isotopes, ${ }^{158} \mathrm{Gd}$ and ${ }^{160} \mathrm{Gd}$, have few resonances and their parameters come from Mughabghab and Chrien ${ }^{10}$ and Rahn et al. ${ }^{13}$ The minority isotopes are ${ }^{152} \mathrm{Gd}$ and ${ }^{154} \mathrm{Gd}$. ${ }^{152} \mathrm{Gd}$ has a natural abundance of $0.2 \%$. Its parameters 
come from Anufriev et al. ${ }^{14}$ and Macklin ${ }^{15} .{ }^{154} \mathrm{Gd}$ has a natural abundance of $2.1 \%$. Its resonance parameters come from References 13 and 15 .

Excellent summaries of published resonance parameters are assembled in the website of the National Nuclear Data Center at the Brookhaven National Laboratory (http://www.nndc.bnl.gov). Of particular interest is the CINDA database (http://www.nndc.bnl.gov /exfor/cinda.htm) which provides extensive lists of papers and reports and summaries of resonance parameters for each isotope.

Many other authors contributed observed resonance energies and/or spin assignments for resonances energies above $148 \mathrm{eV}$ including Belyaev et al. ${ }^{16}$, Karshzhavina et al. ${ }^{17,18}$, and Asghar et al. ${ }^{19}$.

\section{Experimental Conditions}

Neutrons are generated via photoneutron reactions produced by the $\approx 60 \mathrm{MeV}$ pulsed electron beam from the RPI linac. The pulse of electrons strikes a water-cooled tantalum target, where electron collisions generate bremsstrahlung (gamma radiation), which in turn produces photoneutrons. The neutrons are moderated and drift down a long evacuated flight tube to the sample and detector. ${ }^{1,3}$

Table I gives some details of these experiments including neutron targets, overlap filters, linac pulse repetition rate, flight path length, and channel widths.

Two different linac targets were employed in the present series of experiments. Epithermal transmission and capture data were measured using the bare bounce target. ${ }^{4}$ Here, the tantalum target is mounted off of the neutron beam axis, and a $2.54-\mathrm{cm}$-thick polyethylene moderator is mounted adjacent to it on the neutron beam axis. Neutrons scattered in the moderator drift down the flight tube to the detector location. Epithermal measurements were made using an in-beam $0.8-\mathrm{cm}$-thick $\mathrm{B}_{4} \mathrm{C}$ overlap filter to remove any low-energy neutrons from a previous pulse.

Transmission and capture measurements in the thermal energy range utilized the enhanced thermal target (ETT). ${ }^{5,6}$ This target was designed to enhance thermal and subthermal neutron flux. The ETT consists of water-cooled tantalum plates with an integral water moderator, a polyethylene moderator on the axis of the neutron beam, and a graphite reflector.

The neutron energy for a detected event is determined using the TOF technique. The time from the linac electron burst, which creates the neutrons, to the time of the detected event defines the flight time of the neutron. Combining this with precise knowledge of the flight path length gives the neutron energy. The TOF analyzer used in these experiments was designed and constructed in-house, and incorporates a $32 \mathrm{MHz}$ crystal-controlled oscillator coupled to a high speed scaler with 22-bit resolution. It 
Table I

Gadolinium Experimental Details

\begin{tabular}{|c|c|c|c|c|c|c|c|c|c|c|}
\hline Experiment & $\begin{array}{l}\text { Overlap } \\
\text { Filter }\end{array}$ & $\begin{array}{l}\text { Neutron- } \\
\text { Producing } \\
\text { Target }\end{array}$ & $\begin{array}{l}\text { Elec- } \\
\text { tron } \\
\text { Pulse } \\
\text { Width } \\
\text { (ns) }\end{array}$ & $\begin{array}{l}\text { Ave. } \\
\text { Beam } \\
\text { Curr. } \\
(\mu \mathrm{A})\end{array}$ & $\begin{array}{l}\text { Beam } \\
\text { En- } \\
\text { ergy } \\
(\mathrm{MeV})\end{array}$ & $\begin{array}{l}\text { Max Channel } \\
\text { Width }(\mu \mathrm{s})\end{array}$ & $\begin{array}{l}\text { Intermediate } \\
\text { Channel Width } \\
(\mu s)\end{array}$ & $\begin{array}{l}\text { Min } \\
\text { Channel } \\
\text { Width }(\mu \mathrm{s})\end{array}$ & $\begin{array}{l}\text { Pulse } \\
\text { Repeti } \\
\text {-tion } \\
\text { Rate } \\
\text { (pulses } \\
\text { /s) } \\
\end{array}$ & $\begin{array}{l}\text { Fl. } \\
\text { Path } \\
\text { L } \\
\text { (m) }\end{array}$ \\
\hline $\begin{array}{l}\text { Epithermal } \\
\text { Trans_a }\end{array}$ & $\begin{array}{l}\text { Boron } \\
\text { Carbide }\end{array}$ & $\begin{array}{l}\text { Bare } \\
\text { Bounce }\end{array}$ & 160 & 36 & 56 & $\begin{array}{l}0.5 @ \\
<27 \mathrm{eV}\end{array}$ & $\begin{array}{l}0.125 @ \\
27-236 \mathrm{eV}\end{array}$ & $\begin{array}{l}0.0625 \\
@>236 \mathrm{eV}\end{array}$ & 250 & 25 \\
\hline $\begin{array}{l}\text { Epithermal_ } \\
\text { Trans_b }\end{array}$ & Cadmium & $\begin{array}{l}\text { Bare } \\
\text { Bounce }\end{array}$ & 153 & 40 & 50 & $\begin{array}{l}0.5 @ \\
<27 \mathrm{eV}\end{array}$ & $\begin{array}{l}0.125 @ \\
27-236 \mathrm{eV}\end{array}$ & $\begin{array}{l}0.0625 \\
\text { @ }>236 \mathrm{eV}\end{array}$ & 250 & 25 \\
\hline $\begin{array}{l}\text { Thermal_a } \\
\text { Trans } \\
\text { Enriched } \\
\text { Liquids }\end{array}$ & None & $\begin{array}{l}\text { Enhanced } \\
\text { Thermal }\end{array}$ & 1000 & 8 & 53 & $\begin{array}{l}128 @ \\
<0.01 \mathrm{eV}\end{array}$ & $\begin{array}{l}32 @ 0.01-0.04 \mathrm{eV} \\
8 @ 0.04-0.5 \mathrm{eV}\end{array}$ & $\begin{array}{l}0.5 @ \\
>0.5 \mathrm{eV}\end{array}$ & 25 & 15 \\
\hline $\begin{array}{l}\text { Thermal_b } \\
\text { Trans } \\
\text { Enriched } \\
\text { Liquids }\end{array}$ & None & $\begin{array}{l}\text { Enhanced } \\
\text { Thermal }\end{array}$ & 3000 & 19 & 60 & $\begin{array}{l}256 @ \\
<0.008 \mathrm{eV}\end{array}$ & $\begin{array}{l}32 @ \\
0.008-0.04 \mathrm{eV} \\
8 @ 0.04-0.19 \mathrm{eV}\end{array}$ & $\begin{array}{l}1.0 @ \\
>0.19 \mathrm{eV}\end{array}$ & 25 & 15 \\
\hline $\begin{array}{l}\text { Thermal_c } \\
\text { Trans Natrl } \\
\text { Metals }\end{array}$ & None & $\begin{array}{l}\text { Enhanced } \\
\text { Thermal }\end{array}$ & 2100 & 8.5 & 50 & $\begin{array}{l}8 @ \\
<0.29 \mathrm{eV}\end{array}$ & $1 @ 0.29-4.8 \mathrm{eV}$ & $\begin{array}{l}0.5 \\
\text { @ }>4.8 \mathrm{eV}\end{array}$ & 25 & 15 \\
\hline $\begin{array}{l}\text { Epithermal } \\
\text { Capture }\end{array}$ & Cadmium & $\begin{array}{l}\text { Bare } \\
\text { Bounce }\end{array}$ & 128 & 45 & 58 & $\begin{array}{l}2 @ 1.5 \mathrm{eV} \\
<1\end{array}$ & $\begin{array}{l}0.5 @ 1.5-27 \mathrm{eV} \\
0.125 @ \\
27-236 \mathrm{eV}\end{array}$ & $\begin{array}{l}0.0625 \\
@>236 \mathrm{eV}\end{array}$ & 250 & 25 \\
\hline $\begin{array}{l}\text { Thermal } \\
\text { Capture }\end{array}$ & None & $\begin{array}{l}\text { Enhanced } \\
\text { Thermal }\end{array}$ & 3280 & 19 & 52 & $\begin{array}{l}2048 @ \\
<0.02 \mathrm{eV}\end{array}$ & $\begin{array}{l}128 @ 0.02- \\
0.06 \mathrm{eV} \\
16 @ 0.06-0.5 \mathrm{eV}\end{array}$ & $\begin{array}{l}1.0 @ \\
>0.5 \mathrm{eV}\end{array}$ & 25 & 25 \\
\hline
\end{tabular}


operates as a single start/multiple stop device, meaning that a single linac burst initiates a countdown cycle, during which any number of detected events causes the analyzer to record an event. The TOF analyzer employs a fixed deadtime of $0.250 \mu$ s to read each event. The overall deadtime of the signal processing electronics has been set at $1.125 \mu \mathrm{s}$ for capture measurements and $0.6 \mu \mathrm{s}$ for transmission measurements. ${ }^{1}$

The entire data-taking process was controlled by a Hewlett-Packard HP-1000 Model A990 computer. During operation of the experiment, data are transferred from the TOF analyzer to the computer memory via Direct Memory Access. The data-taking software is completely menu driven and controls the sample changer, sorts the data into individual spectra, and provides on-line display of the data being accumulated. Descriptions of the TOF analyzer, data-taking computer system, data file structure, and data reduction process are provided in Reference 1.

Table II lists the isotopic content of the gadolinium samples used in the experiments. The purity of metal samples was $99.8 \%$. The only significant contaminant in the metal samples was tantalum with a manufacturer-specified content of less than $0.1 \%$. The liquid samples were prepared by dissolving enriched gadolinium oxide in $\mathrm{D}_{2} \mathrm{NO}_{3}$, and then diluting in $99.80 \%$ pure $\mathrm{D}_{2} \mathrm{O}$. The uncertainties in isotopic enrichment of liquid samples given in Table II were determined by mass spectroscopy. No evidence of contamination was observed in the liquid sample data.

Table III lists the samples' thicknesses and the measurements made with these samples. The uncertainties in sample thickness for metal samples were propagated from multiple measurements of sample weight and diameter. The diameter measurements were the dominate component of the uncertainties.

All metal samples were natural elemental gadolinium sealed inside aluminum sample cans. The thickness of aluminum on each of the front and rear faces of each sample was $0.38 \mathrm{~mm}(15 \mathrm{mils} ; 1 \mathrm{mil}=0.001 \mathrm{in})$. The influence of these sample cans, as well as all background, was measured by including empty sample cans in the capture measurement. Background in transmission measurements is discussed in Section IV.B.

The liquid samples were enclosed in quartz cells. A drawing of the quartz cell is given in Figure 1. The liquid samples were needed to measure the strong thermal region doublet. The thinnest manufacturable metal sample of uniform thickness was $0.025 \mathrm{~mm}$ thick. At this thickness the thermal region doublet is saturated. The liquid samples provided a uniform solution of enriched $\mathrm{Gd}$ in heavy water. The heavy water minimized non-Gd interactions. The sample container was a quartz cell with parallel inner walls. A $\mathrm{D}_{2} \mathrm{O}$ blank in an equivalent quartz container was included in the liquid sample measurements as a background measurement. 
Table II

Liquid Gadolinium Isotopic Enrichment, Atom Percentages

\begin{tabular}{|l|l|l|l|l|l|l|l|l|}
\hline & Samples & ${ }^{152} \mathrm{Gd}$ & ${ }^{154} \mathrm{Gd}$ & ${ }^{155} \mathrm{Gd}$ & ${ }^{156} \mathrm{Gd}$ & ${ }^{157} \mathrm{Gd}$ & ${ }^{158} \mathrm{Gd}$ & ${ }^{160} \mathrm{Gd}$ \\
\hline $\begin{array}{l}{ }^{155} \mathrm{Gd}- \\
\text { enriched }\end{array}$ & LX-1, LX-2, & 0.0108 & 0.9859 & 74.4233 & 17.5674 & 3.7513 & 2.5336 & 0.7278 \\
\hline${ }^{157} \mathrm{Gd}-$ & LX-4, LX-9 & \pm 0.0002 & \pm 0.0074 & \pm 0.0095 & \pm 0.0051 & \pm 0.0023 & \pm 0.0024 & \pm 0.0020 \\
enriched & LX-7, LX-6, & 0.00510 & 0.07530 & 1.35147 & 7.3627 & 69.6623 & 19.4431 & 2.1000 \\
& LX-11 & \pm 0.00004 & \pm 0.00025 & \pm 0.00070 & \pm 0.0017 & \pm 0.0081 & \pm 0.0067 & \pm 0.0015 \\
\hline Elemental Metals & & 0.2 & 2.2 & 14.8 & 20.5 & 15.7 & 24.8 & 21.9 \\
\hline
\end{tabular}


Table III Elemental Metals and Liquid Isotopic Samples

\begin{tabular}{|c|c|c|c|}
\hline $\begin{array}{l}\text { Metal } \\
\text { Samples }\end{array}$ & $\begin{array}{l}\text { atoms/barn } \\
\text { Gd }\end{array}$ & $\begin{array}{l}\text { Uncertainty } \\
\mathrm{a} / \mathrm{b} \mathrm{Gd}\end{array}$ & Measurements Included \\
\hline $\begin{array}{l}0.025 \mathrm{~mm} \\
(0.001 \mathrm{in})\end{array}$ & $9.119 \mathrm{E}-05$ & $6 \mathrm{E}-08$ & $\begin{array}{l}\text { Thermal and epithermal } \\
\text { transmission } 0.024-10 \mathrm{eV} \text {, } \\
\text { and capture } 1-300 \mathrm{eV}\end{array}$ \\
\hline $\begin{array}{l}0.051 \mathrm{~mm} \\
(0.002 \mathrm{in})\end{array}$ & $1.713 \mathrm{E}-04$ & $1 \mathrm{E}-07$ & $\begin{array}{l}\text { Thermal and epithermal } \\
\text { transmission } 0.055-10 \mathrm{eV}, \\
\text { and capture } 1-300 \mathrm{eV}\end{array}$ \\
\hline $\begin{array}{l}0.127 \mathrm{~mm} \\
(0.005 \mathrm{in})\end{array}$ & $4.127 \mathrm{E}-04$ & $5 \mathrm{E}-07$ & $\begin{array}{l}\text { Thermal and epithermal } \\
\text { transmission } 0.088-300 \mathrm{eV}, \\
\text { and capture } 1-300 \mathrm{eV}\end{array}$ \\
\hline $\begin{array}{l}0.254 \mathrm{~mm} \\
(0.010 \mathrm{in})\end{array}$ & $7.806 \mathrm{E}-04$ & $4 \mathrm{E}-07$ & Epithermal transmission $10-300 \mathrm{eV}$ \\
\hline $\begin{array}{l}0.508 \mathrm{~mm} \\
(0.020 \mathrm{in})\end{array}$ & $1.566 \mathrm{E}-03$ & $5 \mathrm{E}-07$ & Epithermal transmission $10-300 \mathrm{eV}$ \\
\hline $\begin{array}{l}0.889 \mathrm{~mm} \\
(0.035 \mathrm{in})\end{array}$ & $2.886 \mathrm{E}-03$ & $3 \mathrm{E}-06$ & Epithermal transmission $10-300 \mathrm{eV}$ \\
\hline $\begin{array}{l}1.27 \mathrm{~mm} \\
(0.050 \mathrm{in})\end{array}$ & $3.926 \mathrm{E}-03$ & $2 \mathrm{E}-06$ & Epithermal transmission $10-300 \mathrm{eV}$ \\
\hline $\begin{array}{l}2.54 \mathrm{~mm} \\
(0.100 \mathrm{in})\end{array}$ & $8.070 \mathrm{E}-03$ & $2.1 \mathrm{E}-05$ & $\begin{array}{l}\text { Epithermal transmission and capture } \\
10-300 \mathrm{eV}\end{array}$ \\
\hline $\begin{array}{l}5.08 \mathrm{~mm} \\
(0.200 \mathrm{in})\end{array}$ & $1.577 \mathrm{E}-02$ & $3 \mathrm{E}-05$ & Epithermal transmission $10-300 \mathrm{eV}$ \\
\hline $\begin{array}{l}1.02 \mathrm{~cm} \\
(0.400 \mathrm{in}) \\
\end{array}$ & $3.151 \mathrm{E}-02$ & $6 \mathrm{E}-05$ & Epithermal transmission $10-300 \mathrm{eV}$ \\
\hline $\begin{array}{l}\text { Liquid } \\
\text { Samples }\end{array}$ & $\begin{array}{l}\text { atoms/barn } \\
\text { Gd }\end{array}$ & $\begin{array}{l}\text { Uncertainty } \\
\mathrm{a} / \mathrm{b} \mathrm{Gd}\end{array}$ & Measurements Included \\
\hline $\mathrm{LX}-1$ & $1.41 \mathrm{E}-04$ & $\leq 2 \%$ see Note 1 & Thermal transmission $0.047-1.0 \mathrm{eV}$ \\
\hline LX-2 & $4.58 \mathrm{E}-05$ & $\leq 2 \%$ see Note 1 & Thermal transmission $0.006-1.0 \mathrm{eV}$ \\
\hline LX-4 & $1.32 \mathrm{E}-05$ & $\leq 2 \%$ see Note 1 & $\begin{array}{l}\text { Thermal transmission } 0.002-1.0 \mathrm{eV} \text {, } \\
\text { and thermal capture } 0.01-1.0 \mathrm{eV}\end{array}$ \\
\hline LX-5 & $1.27 \mathrm{E}-04$ & $\leq 2 \%$ see Note 1 & Thermal transmission $0.081-1.0 \mathrm{eV}$ \\
\hline $\mathrm{LX}-6$ & $4.34 \mathrm{E}-05$ & $\leq 2 \%$ see Note 1 & Thermal transmission $0.046-1.0 \mathrm{eV}$ \\
\hline LX-7 & $1.19 \mathrm{E}-05$ & $\leq 2 \%$ see Note 1 & Thermal transmission $0.002-1.0 \mathrm{eV}$ \\
\hline $\mathrm{LX}-9$ & $4.39 \mathrm{E}-06$ & $\leq 2 \%$ see Note 2 & Thermal capture $0.01-1.0 \mathrm{eV}$ \\
\hline $\mathrm{LX}-10$ & $3.97 \mathrm{E}-06$ & $\leq 2 \%$ see Note 2 & Thermal capture $0.01-1.0 \mathrm{eV}$ \\
\hline $\mathrm{LX}-11$ & $1.32 \mathrm{E}-06$ & $\leq 2 \%$ see Note 2 & Thermal capture $0.01-1.0 \mathrm{eV}$ \\
\hline
\end{tabular}

Note 1 - In the chemical preparation of the liquid samples, the uncertainty in sample thickness is limited by the two required measurements of volume, each with an estimated uncertainty of $0.2 \%$. The quadrature sum error is $\sqrt{ }\left(2 \mathrm{x}^{2}\right) \approx 0.3 \%$. However, thicknesses determined in this manner have been found to be discrepant by $2 \%$ (see Section VII.A).

Note 2 - These three samples, LX-9, LX-10, and LX-11, were further diluted and therefore required a third measurement of volume with an uncertainty of $0.2 \%$. 


\section{III.A. Capture Detector}

The capture detector is a multiplicity-type scintillation gamma detector containing 16 sections of $\mathrm{NaI}(\mathrm{Tl})$, formed into a 30.5 -cm diameter $\times 30.5$-cm length (12-in $\times 12$-in) right circular cylinder with a $8.9-\mathrm{cm}\left(3.5\right.$-in) through hole along its axis. ${ }^{1,2,3}$ The cylinder is split normal to its axis into two rings, and each ring is divided into 8 equal pie-shaped segments. Each segment is optically isolated in a hermetically sealed aluminum can and is mounted on an RCA 8575 photomultiplier tube. The total volume of $\mathrm{NaI}(\mathrm{Tl})$ is 20 liters. The capture detector used for the present measurements was located at the East beam tube at a flight path 25.564 meters from the bare bounce target for epithermal measurements. This flight path length was determined from measurements of preciselyknown uranium resonance energies. ${ }^{21}$ The capture detector is located $25.44 \mathrm{~m}$ from the ETT for thermal measurements. This flight path length was also determined from uranium data. The efficiency of the capture detector is assumed to be the same for all $\mathrm{Gd}$ isotopes.

All samples were positioned at the center of the detector by a computer-controlled sample changer. The sample changer accommodates up to eight samples, and cycles them into the beam one at a time. The metal samples were $5.08 \mathrm{~cm}$ in diameter and the neutron beam was collimated to $4.76 \mathrm{~cm}$. Neutrons that scatter from the sample are absorbed by a hollow cylindrical liner fabricated of boron carbide ceramic to reduce the number of scattered neutrons reaching the detector. The liner uses boron enriched to 98.4 $\mathrm{w} / \mathrm{o}^{10} \mathrm{~B}$ for maximum neutron absorption. The liquid samples were $1.27-\mathrm{cm}$ in diameter and the neutron beam was collimated to $1.11 \mathrm{~cm}$. The final collimator used for the liquid sample measurements was an annular cylinder of ${ }^{10} \mathrm{~B}$ powder placed inside the detector just a few centimeters from the samples. The detector system discriminates against the $478 \mathrm{keV}$ gamma ray from ${ }^{10} \mathrm{~B}(\mathrm{n}, \alpha)$ reactions. A 15 -cm (6-in)-thick, $7260-\mathrm{kg}(16,000$-lb) lead shield surrounds the detector to reduce the gamma-ray background. Reference 1 contains a description of the detector and its signal processing electronics.

\section{III.B. Transmission Detectors}

Neutron transmission measurements were conducted at the 15-meter and 25-meter flight stations. The 15 -meter station contains a 7.62-cm (3-in) diameter, 0.3 -cm-thick NE $905^{6} \mathrm{Li}$ glass scintillation detector ( $6.6 \%$ lithium, enriched to $95 \%$ in ${ }^{6} \mathrm{Li}$ ) and is typically used for measurements covering the energy range from $0.001 \mathrm{eV}$ to $20 \mathrm{eV}$. The 25 -meter station contains a $12.70-\mathrm{cm}(5-\mathrm{in})$ diameter, $1.27-\mathrm{cm}$-thick NE $905^{6} \mathrm{Li}$ glass detector and covers the range from $1 \mathrm{eV}$ to $500 \mathrm{eV}$.

Each detector is coupled to a photomultiplier tube, which is in line with the flight path. The transmission detector used for the epithermal measurements was located at the center beam tube at a flight path of 25.585 meters from the bare bounce target. The flight path for thermal transmission measurements was $14.973 \mathrm{~m}$. The thermal transmission flight path length was deduced from measurement of known uranium resonances. ${ }^{21}$ 
Transmission samples along with empty sample holders, which are used to measure the open-beam count rate, are mounted on an 8-position computer-controlled sample changer. The transmission function, which is approximately the ratio of the count rate with a sample in the beam to the count rate with samples removed, varies with neutron energy. Each data run consists of one complete cycle through the samples, with a predetermined number of linac bursts for each sample position. The distribution of bursts per sample position is chosen to minimize the counting statistical error in the measured cross section. ${ }^{22}$

\section{Data Reduction}

\section{IV.A. Capture Data}

A capture experiment typically involves taking several kinds of data. Sample data are taken with up to seven samples mounted on the sample changer. These usually include about four samples of different thickness, a sample to be used for flux normalization, and two or three empty sample holders for background evaluation. Usually 32 TOF spectra are measured for each sample, i.e., 16 spectra of capture data, and 16 spectra of scattering data, resulting in a data file size of approximately 8 megabytes per run. A minimum of $100 \mathrm{keV}$ gamma energy is required in a detector segment for the data to be counted. Data are recorded as capture events if the total energy deposited in all 16 detector segments exceeds $1 \mathrm{MeV}$ for epithermal and liquid measurements or $2 \mathrm{MeV}$ for thermal measurements. These discriminator settings have been found to reduce background from ${ }^{128}$ I decay. Data are recorded as scattering events if the total gamma energy deposited falls between 360 and $600 \mathrm{keV}$. This energy region contains the $478 \mathrm{keV}$ gamma emitted following an $(\mathrm{n} ; \alpha, \gamma)$ reaction in the ${ }^{10} \mathrm{~B}_{4} \mathrm{C}$ annular detector liner.

The linac electron burst widths and the channel widths for the Gd experiments are summarized in Table I. Low-energy channels were grouped into fewer, wider channels to accumulate better statistics per data point. The average accelerator current during each experiment is also given in Table I. Each of these measurements lasted approximately 72 hours. In order to minimize data loss in the event of an equipment malfunction and to average linac beam-intensity fluctuations an individual run duration of about one hour was used. Multiple runs were summed off-line to accumulate the necessary statistical accuracy.

Once accumulated, the time-of-flight data were subjected to a "consistency check" in which data were accepted only when the recorded detector counts and beam monitor counts fell within a range of statistical fluctuations. Any data where malfunctions occurred were eliminated. Next, all data were dead-time corrected, similar data sets were combined into composite data runs, and these were normalized to the neutron beam fluence using fixed monitor detectors on the same or adjacent beam tubes. Background was determined using normalized data measured with an empty sample holder mounted on the sample changer. 
For the thermal measurement of liquid samples, background was determined using a cell containing $\mathrm{D}_{2} \mathrm{O}$. Background spectra were then subtracted from the normalized, summed spectra. Finally, the 16 individual multiplicity spectra were summed into a single multiplicity-independent total spectrum.

Processed capture data are expressed as yield. Yield is defined as the number of neutron captures per neutron incident on the sample. Therefore, in addition to the sample data, another set of data was needed to determine the energy profile of the neutron flux. This was done by mounting a thick ${ }^{10} \mathrm{~B}_{4} \mathrm{C}$ sample in the sample changer and adjusting the total energy threshold to record the $478 \mathrm{keV}$ gamma rays from neutron absorption in ${ }^{10} \mathrm{~B}$. The boron absorption spectrum provides an accurate representation of the energy profile of the linac's neutron beam flux convoluted with the $1 / \mathrm{v}$ boron $(n, \alpha)$ cross section. These flux data give the shape of the neutron beam flux, but not its magnitude. The thermal flux was normalized to the black ( $100 \%$ absorption) region below $0.01 \mathrm{eV}$ in a $0.025-\mathrm{mm}$ (1-mil) natural metal Gd sample. This metal normalizing sample's diameter was $5.08 \mathrm{~cm}$ (2-in), larger than that of the liquid samples. A geometric correction factor of 1.021 was divided into the observed count rate in the normalizer. The factor was determined from a measurement using $1.27-\mathrm{cm}(0.5$-in)-diameter and $5.08-\mathrm{cm}$ (2-in) diameter black cadmium samples The thermal flux shape was also smoothed using a cubic spline interpolation. The epithermal flux was normalized to the black $6.3-\mathrm{eV}$ predominantlycapture resonance in Gd. A small correction (1.8\%) was made for the scattering in the $6.3-\mathrm{eV}$ normalizing resonance. The $2.54-\mathrm{mm}(100-\mathrm{mil})$ sample data were used for this normalization.

The zero time for each experiment was determined by performing a 'gamma flash' measurement. The burst of gamma rays accompanying the neutron burst is detected by the capture detector. The centroid of the gamma-flash peak, less the time for light to travel the length of the flight path, is defined as the zero time of neutron production.

Finally, $Y_{i}$, the capture yield in time-of-flight channel $i$, was calculated by:

$$
Y_{i}=\frac{C_{i}-B_{i}}{K \phi_{i}}
$$

where $C_{i}$ is the dead-time-corrected and monitor-normalized counting rate of the sample measurement, $\mathrm{B}_{\mathrm{i}}$ is the dead-time-corrected and monitor-normalized background counting rate, $\mathrm{K}$ is the product of the flux normalization factor and efficiency, and $\Phi_{\mathrm{i}}$ is the unnormalized neutron flux.

Separate beam-monitor detectors including a ${ }^{6} \mathrm{Li}$ glass scintillation detector and two ${ }^{235} \mathrm{U}$ fission chambers were used to monitor neutron intensity. The capture yield for each sample was produced by dividing its monitor-normalized and background-corrected capture spectrum by the normalized neutron flux spectrum. It was this capture yield and 
its associated statistical uncertainty that provided input to the SAMMY ${ }^{7}$ data analysis code that extracted the neutron resonance parameters.

Four liquid capture samples were used in the analysis of the thermal region. The low energy cutoff for capture data in the thermal region was $0.01 \mathrm{eV}$. Four natural metal samples were used in the epithermal region, $1-300 \mathrm{eV}$.

Flux-to-background ratios for the liquid sample thermal capture experiment are given in Figure 2. The ratio in the region of the two strong thermal resonances in Gd is greater than 10-to-1. Flux-to-background ratios for the natural metal epithermal capture experiment are given in Figure 3. The ratio is greater than 100-to-1 throughout most of the epithermal resonance region.

\section{IV.B. Transmission Data}

The linac electron pulse width, channel widths, and average accelerator current for the Gd experiments are summarized in Table I. Each of these experiments lasted approximately 72 hours.

As with the capture measurements, a transmission measurement consists of many individual runs of about one-hour in duration which are summed off-line to provide the necessary statistical accuracy. Two sample changer positions were used to measure open beam count rate, and these were placed at the beginning and middle of each sample cycle. The time split between samples and open beam was optimized to minimize the error in the cross section. ${ }^{22}$ For the thermal measurement, liquid sample cells containing $\mathrm{D}_{2} \mathrm{O}$ were used as the open beam measurement. In this way the effect of the $\mathrm{D}_{2} \mathrm{O}$ and quartz in the sample and open cells would cancel.

The large amount of data collected in each of the experiments was first run through a statistical consistency check, as discussed earlier, to verify the stability of the linac, the in-beam detector, and the beam monitors. The data were then corrected for dead-time, and runs were normalized and summed.

The time-dependent background was obtained with the one-notch/two-notch method. ${ }^{23}$ This method employs blacked-out notch filters of single and double material thickness, and the resulting background determined from each was extrapolated to a zero effective material thickness. The measured background was then fitted to a smooth analytical function of time-of-flight for each sample, including the open beam measurement. The resulting analytical expression was used for the background correction. Finally, the transmission function was calculated from equation (2).

$$
T_{i}=\frac{\left(C_{i}^{S}-K_{S} B_{i}-B_{S}\right)}{\left(C_{i}^{o}-K_{o} B_{i}-B_{o}\right)}
$$


where

$T_{i}$, the transmission in time-of-flight channel $\mathrm{i}$, $C_{i}^{S}$ and $C_{i}^{o}$ are the dead-time corrected and monitor-normalized counting rates of the sample and open measurements, respectively,

$B_{i}$ is the time-dependent background counting rate,

$B_{S}$ and $B_{O}$ are the steady state background counting rates for sample and open measurements, respectively, and

$K_{S}$ and $K_{O}$ are the normalization factors for the sample and open background measurements.

The first thermal liquid sample measurement's background was normalized to the extrapolated notch in Cd at $0.24 \mathrm{eV}$. Since this point is not at the peak of the cadmium cross section, a later evaluation of the background fit was performed with a normalization in the $0.15-0.17 \mathrm{eV}$ region of the $\mathrm{Cd}$ notch and no significant differences resulted; i.e., results were within quoted uncertainties.

The second thermal liquid sample measurement's background was normalized to the extrapolated notch in $\mathrm{Cd}$ at $0.15 \mathrm{eV}$.

For the thermal metal measurement, a single exponential function was used to interpolate between two known background points: a fixed indium notch at $1.4 \mathrm{eV}$ and the region below $0.01 \mathrm{eV}$, where all metal Gd samples are black. Normalization constants $\mathrm{K}_{\mathrm{S}}$ and $\mathrm{K}_{\mathrm{O}}$ were fixed at 1.0 for the thermal metal measurement. Each sample's background function was calculated individually.

The first epithermal measurement's background was normalized to the extrapolated notch in silver at $5.2 \mathrm{eV}$ for all samples except the 1.02-cm $(400-\mathrm{mil})$ sample, which was normalized at the saturated $20.5 \mathrm{eV}$ resonance in Gd.

The second epithermal measurement's background was normalized to the extrapolated notch in tungsten at $18.8 \mathrm{eV}$.

The epithermal flight path length $(\approx 25.6 \mathrm{~m})$ and zero time were fitted to match the energies from epithermal capture data.

The flight path of the thermal measurements was $14.973 \mathrm{~m}^{24}$ The zero-time for each experiment was determined by performing a 'gamma flash' measurement. The burst of gamma rays accompanying the neutron burst is detected by the ${ }^{6} \mathrm{Li}$ glass detector. The centroid of the gamma-flash peak, less the travel time for light to travel the length of the flight path, is defined as the zero time of neutron production.

Seven liquid transmission data sets were used in the thermal analysis. Three natural metal samples were used in the thermal and epithermal energy ranges up to 10 $\mathrm{eV}$. Eleven additional natural metal transmission data sets were used in the epithermal analysis from $1-300 \mathrm{eV}$. 
Signal-to-background ratios for the two liquid sample thermal experiments are given in Figure 4. The ratio in the region of the two strong thermal resonances in $\mathrm{Gd}$ is greater than 2000-to-1. Signal-to-background ratios for the metal sample thermal experiment are given in Figure 5. The ratio in the region of the two strong thermal resonances in Gd is approximately 1000-to-1. These data were also analyzed in the epithermal resonance region up to $10 \mathrm{eV}$ where the signal-to-background ratio was approximately 200-to-1. Signal-to-background ratios for the two metal sample epithermal experiments are given in Figure 6. The ratios in the epithermal resonance region are rather constant with energy at approximately 45-to-1 for the boron-filtered experiment and approximately 75 -to-1 for the cadmium-filtered experiment.

\section{IV.B.1 Transmission Data- Accounting for Open Beam Leakage}

Three of the liquid transmission samples (LX-1,5, and 6) were black at subthermal energies (See Figure 7). These data appear to have reached a constant value somewhere above zero transmission. If the samples are truly black, the count rate in the sample data (before being divided by the flux to calculate transmission) should be zero. Figure 8 shows that the sample-in counting rates for these samples are not zero in the region $0.0-0.02 \mathrm{eV}$ where transmissions are constant (Figure 7). When the open beam measurement is compared to the shape of these sample-in count rates (Figure 8), there is a remarkable agreement in shape. It is believed that a small physical misalignment of the samples with respect to the beam centerline occurred. When these fractions of open beam are subtracted from the transmission data, each sample's transmission reduces to a constant value of zero in the subthermal region. The fractions of beam "leaking by" these samples were LX-1: $0.15 \%$ of open, LX-5: $1.3 \%$ of open, LX-6: $0.56 \%$ of open. These three fraction-open-beam-corrected data sets were used in the SAMMY analysis. No other samples were adjusted for this problem.

\section{Analysis Methods}

Resonance parameters, neutron width, $\Gamma_{\mathrm{n}}$, radiation width, $\Gamma_{\gamma}$, and resonance energy, $E_{0}$,were extracted from the capture and transmission data sets using the multilevel R-matrix Bayesian code SAMMY version $\mathrm{M}^{7}$. This was a combined transmission and capture analysis, which employed the resolution broadening, self-shielding, multiplescattering, and diluent features of SAMMY .

Resolution broadening refers to the combined effects of the linac electron burst width, the time delay in the moderator, the TOF channel width, and the effect of the detector system. Burst width and channel width are entered as SAMMY input parameters. All other components of the resolution function are handled collectively as an analytical function of amplitude versus time whose integral over time is unity. The function describing the RPI linac experimental configuration is now an explicit option in SAMMY. ${ }^{7}$ 
In capture, the resolution function is modeled as a Gaussian pulse with an exponential tail. The width of the Gaussian is that of the pulse of neutrons measured in the 'gamma flash' experiment. The tail is characterized by an exponential folding width in $\mu \mathrm{s}$, described as variable DELTAE in Reference 7, Section IV.A.2.b. A value of 0.060 $\mu$ s was used for the DELTAE variable in SAMMY for both thermal and epithermal data. $^{25}$

In transmission the resolution function used was the function specifically included in the SAMMY code for the RPI linac facility. The RPI resolution function parameters, as determined by Reference 25 , were used in the present analysis for both thermal and epithermal data.

The self-shielding correction accounts for the attenuation of the incident beam in the sample. The multiple-scattering correction accounts for the increase in observed capture yield due to capture of neutrons scattered from higher energies. Both corrections were applied in SAMMY for all capture analyses. Thick sample data were used only when a resonance had a poor signal-to-background ratio in thinner sample data.

The SAMMY geometry does not allow layers; it must consist of a homogeneous mixture of materials. In SAMMY, the $\mathrm{Gd}$ and $\mathrm{D}_{2} \mathrm{O}$ are modeled, but not the quartz cell. The three-dimensional geometry in $\mathrm{MCNP}^{26}$ allowed a realistic treatment of the neutron scattering and capture in $\mathrm{Gd}, \mathrm{D}_{2} \mathrm{O}$ and quartz cell geometry. Two models were made to simulate the data. The data are the difference between the yields of a quartz cell containing a $\mathrm{Gd}$ and $\mathrm{D}_{2} \mathrm{O}$ mixture and a cell containing only $\mathrm{D}_{2} \mathrm{O}$. A third model was made to mimic the geometrical representation in SAMMY, which is $G d$ and $D_{2} O$ in a homogeneous mixture with no quartz. The ratio of the MCNP-calculated capture yields in the homogeneous slab geometry to those of the data simulation vs energy is an estimate of the fraction of captures due to quartz-to-gadolinium scattering (see Figure 9). It was used as a correction factor on the measured capture yields. A typical distribution of this factor with energy is shown in Figure 9 for sample LX-4. More detail is provided in Appendix A.

For liquid thermal transmission analysis, no diluent, i.e., $\mathrm{D}_{2} \mathrm{O}$, specifications are needed. That is because $\mathrm{D}_{2} \mathrm{O}$-only "blank" cells are used for the open beam measurement and therefore the effect of the diluent cancels experimentally from the transmission, defined as (quartz + sample + diluent) / (quartz + diluent-only open beam). Therefore, the SAMMY model includes only Gd. The presence of $\mathrm{Gd}$ in the samples is so dilute that there is no need to account for $\mathrm{D}_{2} \mathrm{O}$ displaced by the $\mathrm{Gd}$.

Table IV summarizes the Gd evaluations from ENDF/B-VI ${ }^{8}$ updated through release 8. These are the latest Gd evaluations. Whenever the term ENDF is used in this report, refer to Table IV for the specific evaluation used for each isotope. As a starting point, SAMMY utilized Gd parameters from the evaluations listed in Table IV. 
Table IV Summary of Gadolinium ENDF Evaluations

\begin{tabular}{|c|c|}
\hline Gadolinium Isotope & Latest ENDF Evaluation \\
\hline 152 & ENDF6.8 \\
\hline 154 & ENDF6.8 \\
\hline 155 & ENDF6.8 \\
\hline 156 & ENDF6.0 \\
\hline 157 & ENDF6.8 \\
\hline 158 & ENDF6.0 \\
\hline 160 & ENDF6.0 \\
\hline
\end{tabular}

The following assumptions were made for the SAMMY analysis:

- Background was not fitted during the SAMMY analysis which determined the final resonance parameters. Background was only varied in transmission in the $10-80 \mathrm{eV}$ region as a sensitivity study for the purpose of determining uncertainties (see Section VII and Appendix B).

- Resonance parameters of the two bound level resonances of $\mathrm{Gd}\left({ }^{152} \mathrm{Gd}\right.$ and $\left.{ }^{154} \mathrm{Gd}\right)$ were not varied.

- Normalization was not varied for either capture or transmission in the epithermal region.

- Energy regions over which each sample has been fitted are given in Table III. Low energy cutoffs were chosen at a point where transmission falls below $1 \%$. The thermal flux peaks at $\approx 0.08 \mathrm{eV}$ and drops off rapidly with decreasing energy. The combination of low flux and the highly absorbing nature of gadolinium at subthermal energies led to regions of low transmission where the accuracy of the background treatment is important.

- There were concerns about background in both epithermal transmission measurements using metal samples below $80 \mathrm{eV}$, and particularly below $10 \mathrm{eV}$. Therefore, the lowenergy cut-off for these data sets was $10 \mathrm{eV}$.

The potential scattering lengths used in the present analysis for gadolinium are given in the "Initial values" column of Table V. They are taken from ENDF. The potential scattering lengths for each of the gadolinium isotopes except ${ }^{152} \mathrm{Gd}$ were varied with SAMMY to obtain a better fit to the thick $(5.08-\mathrm{mm})$ transmission data. The resulting potential scattering lengths are given in Table $\mathrm{V}$. The initial values listed in the table were used for the resonance parameter analysis.

The radius to be used for penetrabilities and shifts were calculated using equation

$$
\mathrm{a}=23 * \mathrm{AWRI}^{1 / 3}+0.8
$$


Table V - Potential Scattering Lengths for Gadolinium Isotopes

\begin{tabular}{|c|c|c|}
\hline Isotope & $\begin{array}{c}\text { Initial values from ENDF and } \\
\text { SAMMY }(\mathrm{fm})\end{array}$ & $\begin{array}{c}\text { Final Potential Scattering } \\
\text { Lengths }(\mathrm{fm})\end{array}$ \\
\hline${ }^{152} \mathrm{Gd}$ & 8.2 & not varied \\
\hline${ }^{154} \mathrm{Gd}$ & 8.0 & 8.01 \\
\hline${ }^{155} \mathrm{Gd}$ & 8.0 & 8.08 \\
\hline${ }^{156} \mathrm{Gd}$ & 8.1 & 8.25 \\
\hline${ }^{157} \mathrm{Gd}$ & 7.8 & 7.74 \\
\hline${ }^{158} \mathrm{Gd}$ & 6.5 & 6.53 \\
\hline${ }^{160} \mathrm{Gd}$ & 6.8 & 6.85 \\
\hline
\end{tabular}

where,

$a$ is the channel radius, and

AWRI is the atomic weight given in the ENDF file. This atomic weight is based on the mass of the neutron rather than amu.

The potential scattering lengths used for deuterium and oxygen in the liquid samples were $5.20 \mathrm{fm}$ and $5.46 \mathrm{fm}$, respectively. These radii were calculated from potential scattering cross-sections, deuterium $\sigma_{\mathrm{s}}=3.4$ barns, oxygen $\sigma_{\mathrm{s}}=3.75$ barns. $^{27}$ The effective temperature was $293 \mathrm{~K}$, and no external R-function was employed. Distant resonances were represented by including all of the resonances present in the ENDF file. No p-wave resonances were observed in the energy range currently being reported. The calculations determined the resonance energy, $E_{0}$, and the resonance widths, $\Gamma_{n}$ and $\Gamma_{\gamma}$. The present measurements assumed the same spin assignments as ENDF for all resonances analyzed.

The manufacturer-specified tantalum content of the natural metal samples was less than $0.1 \%$. A SAMMY fit of thick samples to the strong $4.28 \mathrm{eV}$ resonance in tantalum yielded an abundance of $0.13 \%$ which was used in all resonance parameter fits. That is, all ENDF resonances for Ta were included in all metal-sample SAMMY calculations with an abundance of $0.13 \%$.

Resonance integrals are defined in equation (4).

$$
R I=\int_{0.5 e V}^{\infty} \sigma_{C}(E) \cdot \frac{d E}{E}
$$

where $R I$ is the infinitely dilute capture resonance integral, in barns, and $\sigma_{C}(E)$ is the neutron capture cross section in barns 
Resonance integrals and thermal cross sections were calculated using the NJOY ${ }^{28}$ program. The resonance integrals were evaluated from $0.5 \mathrm{eV}$ to $20 \mathrm{MeV}$. One calculation was performed using ENDF resonance parameters and one calculation was performed with RPI resonance parameters replacing the ENDF parameters for all resonances below $300 \mathrm{eV}$. Results are presented in Section VI.C.

VI. Results

\section{VI.A Results- Thermal}

Resonance parameters were varied in a covariance-matrix-linked calculation using the SAMMY code. The resulting resonance parameters for the two thermal region resonances are given in Table VI. ENDF/B-VI resonance parameters are nearly identical to those of Moller et al. ${ }^{9}$ The uncertainties given in Reference 9 are reported as ENDF uncertainties in Table VI. The uncertainties given in Table VI for the thermal region were estimated to be on the order of $1 \sigma$ and include the following considerations: internal consistency of the transmission data, reproducibility of transmission results, the uncertainty in capture flux normalization, and the balance of interactions between the overlapping ${ }^{155} \mathrm{Gd}$ and ${ }^{157} \mathrm{Gd}$ thermal resonances. The methods used to estimate the KAPL/RPI uncertainties are discussed in Section VII.A and Appendix B. Since both of these resonances are predominantly capture resonances, both transmission and capture measurements were essentially measuring capture. The neutron width is the resonance parameter that was most sensitive to the data in both capture and transmission. Neutron widths for both resonances were found to be smaller than ENDF, by $9 \%$ in the ${ }^{157} \mathrm{Gd}$ resonance and by $7 \%$ in the smaller ${ }^{155} \mathrm{Gd}$ resonance.

Fourteen samples were included in this calculation: seven liquid transmission samples ( 4 enriched in ${ }^{155} \mathrm{Gd}, 3$ enriched in ${ }^{157} \mathrm{Gd}$ ), three natural metal transmission samples, and four liquid capture samples ( 2 enriched in ${ }^{155} \mathrm{Gd}, 2$ enriched in ${ }^{157} \mathrm{Gd}$ ). No natural metal capture measurements were made. Capture yield data were normalized to transmission by including capture normalization as a variable in the fit. The resulting capture normalization factor was 0.9 times the initial estimate described in Section IV (Data Reduction). Results of the thermal fit are shown in Figure 10 - Figure 16.

Figure 10 shows the $0.051 \mathrm{~mm}(2-\mathrm{mil})$ metal sample in transmission. The fit shows better agreement to the current data than that from ENDF parameters below 0.1 $\mathrm{eV}$. The thermal-region ${ }^{157} \mathrm{Gd}$ resonance is approximately four times stronger than the ${ }^{155} \mathrm{Gd}$ resonance and their relative abundances in natural metal are approximately the same (see Table II). Figure 11 shows the SAMMY fit to a ${ }^{155} \mathrm{Gd}$-enriched liquid sample in transmission, LX-4 (See Table II). Figure 12 shows the SAMMY fit to a ${ }^{157} \mathrm{Gd}$ enriched liquid sample in transmission, LX-7 (See Table II).

Figure 13 shows the SAMMY fit to a "thick" ${ }^{155}$ Gd-enriched liquid sample in transmission, LX-1 (See Table II). The data shown have been corrected for the $0.15 \%$ open beam leakage discussed in Section IV.B.1. Figure 14 shows the SAMMY fit to a 
Table VI

Thermal Results: Resonance Parameters

\begin{tabular}{|c|c|c|c|c|c|}
\hline Dataset & Energy, eV & $\Gamma_{\gamma}, \mathrm{meV}$ & $\Gamma_{\mathrm{n}}, \mathrm{meV}$ & ISOTOPE & $\mathrm{J}$ \\
\hline ENDF- & $0.0268 \pm 0.0002$ & $108 \pm 1$ & $0.104 \pm 0.002$ & 155 & 2 \\
\cline { 2 - 6 } B/VI & $0.0314 \pm 0.0002$ & $106 \pm 1$ & $0.4704 \pm 0.0080$ & 157 & 2 \\
\hline \multirow{2}{*}{ RPI } & $0.025 \pm 0.003$ & $104 \pm 3$ & $0.097 \pm 0.003$ & 155 & 2 \\
\cline { 2 - 7 } & $0.032 \pm 0.003$ & $107 \pm 3$ & $0.428 \pm 0.004$ & 157 & 2 \\
\hline
\end{tabular}

"thick" ${ }^{157}$ Gd-enriched liquid sample in transmission, LX-6 (See Table II). The data shown have been corrected for the $0.56 \%$ open beam leakage discussed in Section IV.B.1. The inability of a single set of resonance parameters to fit all data sets simultaneously is due to internal inconsistencies in the data.

Figure 15 shows the SAMMY fit to a ${ }^{155} \mathrm{Gd}$-enriched liquid sample in capture, LX-4 (See Table II). Notice that the SAMMY fit to these data is actually poorer than that from ENDF parameters. This is a consequence of the internal inconsistencies between data sets. ${ }^{157} \mathrm{Gd}$ neutron width is determined predominantly from natural metal transmission data and ${ }^{157} \mathrm{Gd}$-enriched liquid sample transmission data. Capture normalization, in turn, is determined predominantly by the transmission-derived ${ }^{157} \mathrm{Gd}$ neutron width. The ${ }^{155} \mathrm{Gd}$ neutron width is determined predominantly by ${ }^{155} \mathrm{Gd}$-enriched liquid transmission data, and to a lesser extent, by natural metal transmission data and ${ }^{155} \mathrm{Gd}$-enriched liquid sample capture data. In the present data, transmission data have smaller statistical uncertainties than capture yield data, and thick samples have more influence on derived resonance parameters than thin samples in both capture and transmission. ${ }^{22}$ The fact that a unique set of two neutron widths and one capture normalization factor could not be found that matched all data sets is addressed in the discussion of uncertainties in the next section (Section VII). Figure 16 shows the SAMMY fit to a ${ }^{157}$ Gd-enriched liquid sample in transmission, LX-11 (See Table II). The figure shows that the capture normalization factor $(0.9)$ has been chosen to match the ${ }^{157} \mathrm{Gd}$-enriched data (Figure 16) using the neutron width of the dominant resonance $\left(0.031 \mathrm{eV}\right.$ in $\left.{ }^{157} \mathrm{Gd}\right)$, determined primarily from transmission data.

\section{VI.B Results- Epithermal}

The epithermal region was analyzed in two parts. First the $1-10 \mathrm{eV}$ region was fitted with a combination of thermal transmission and epithermal capture data sets (see Section V). Second, the $10-300 \mathrm{eV}$ region was fitted using data entirely from natural metal samples in capture and transmission. Resonance parameters for the epithermal region are given in Table VII. The uncertainties quoted in Table VII for the epithermal region were estimated to be on the order of $1 \sigma$, are described in Section VII.B and Appendix B, and include the following considerations: Consistency between capture and transmission results, stability of radiation widths, uncertainty in transmission background treatment, and Bayesian statistical errors. There are 28 new resonances introduced that 
Table VII Epithermal Results: Resonance Parameters

\begin{tabular}{|c|c|c|c|c|c|c|c|c|c|c|c|c|}
\hline \multirow[b]{2}{*}{2.0120} & \multirow{2}{*}{\multicolumn{3}{|c|}{$\begin{array}{c}\text { Energy } \\
\text { ENDF } \\
\text { meV } \\
2.008\end{array}$}} & \multirow[b]{2}{*}{128} & \multicolumn{2}{|c|}{$\begin{array}{c}\Gamma_{\mathrm{Y}} \\
\mathrm{meV}\end{array}$} & \multirow[t]{2}{*}{ 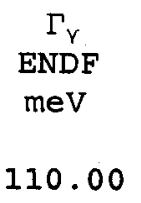 } & \multicolumn{2}{|c|}{$\begin{array}{c}\Gamma_{\mathrm{n}} \\
\text { (2ag } \Gamma_{\mathrm{n}} \text { for } \\
\text { unassigned) } \\
\text { meV }\end{array}$} & \multirow{2}{*}{$\begin{array}{c}\Gamma_{\mathrm{n}} \\
\text { ENDF } \\
\mathrm{meV} \\
0.371\end{array}$} & \multirow[t]{2}{*}{$\begin{array}{l}\text { ISO- } \\
\text { TOPE } \\
\text { ENDF } \\
155\end{array}$} & \multirow{2}{*}{$\begin{array}{l}\text { J } \\
\text { ENDF } \\
1\end{array}$} \\
\hline & & & & & \pm & 1 & & 0.40 & \pm 0.01 & & & \\
\hline 2.5729 & \pm & 0.0003 & 2.568 & 107.1 & \pm & 0.4 & 111.00 & 1.706 & \pm 0.003 & 1.744 & 155 & 2 \\
\hline 2.8287 & \pm & 0.0003 & 2.825 & 109.7 & \pm & 0.9 & 97.00 & 0.377 & \pm 0.004 & 0.345 & 157 & 2 \\
\hline 3.616 & \pm & 0.003 & 3.616 & 130 & & & 130.00 & 0.05 & \pm 0.02 & 0.044 & 155 & 1 \\
\hline 6.3057 & \pm & 0.0002 & 6.300 & 108.8 & \pm & 0.6 & 114.00 & 2.20 & \pm 0.01 & 2.000 & 155 & 2 \\
\hline 7.7477 & \pm & 0.0004 & 7.750 & 109 & \pm & 1 & 124.00 & 1.16 & \pm 0.01 & 1.120 & 155 & 2 \\
\hline 9.991 & \pm & 0.003 & 10.010 & 110 & \pm & 20 & 115.00 & 0.20 & \pm 0.04 & 0.168 & 155 & 2 \\
\hline 11,508 & \pm & 0.001 & 11.530 & 120 & \pm & 40 & 125.00 & 0.78 & \pm 0.08 & 0.600 & 155 & 1 \\
\hline 11.57 & \pm & 0.05 & 11.580 & 90 & \pm & 80 & 88.00 & 0.2 & \pm 0.2 & 0.350 & 154 & $1 / 2$ \\
\hline 11.964 & \pm & 0.008 & 11.990 & 130 & \pm & 20 & 112.00 & 1.12 & \pm 0.04 & 0.880 & 155 & 2 \\
\hline 12.35 & & & 12.350 & 58.6 & & & 58.60 & 4.65 & & 4.650 & 152 & $1 / 2$ \\
\hline 14.476 & \pm & 0.009 & 14.510 & 130 & \pm & 10 & 103.00 & 3.43 & \pm 0.09 & 3.200 & 155 & 1 \\
\hline 16.201 & \pm & 0.005 & 16.240 & 130 & \pm & 30 & 91.00 & 0.44 & \pm 0.03 & 0.400 & 157 & 1 \\
\hline 16.78 & \pm & 0.01 & 16.770 & 112 & \pm & 7 & 80.50 & 13.9 & \pm 0.5 & 12.800 & 157 & 2 \\
\hline 17.729 & \pm & 0.005 & 17.770 & 130 & \pm & 40 & 120.00 & 0.47 & \pm 0.04 & 0.392 & 155 & 2 \\
\hline 19.86 & \pm & 0.01 & 19.920 & 118 & \pm & 6 & 104.00 & 4.5 & \pm 0.1 & 4.560 & 155 & 2 \\
\hline 20.51 & \pm & 0.02 & 20.560 & 106 & \pm & 8 & 88.00 & 13.4 & 0.4 & 11.360 & 157 & 2 \\
\hline 20.97 & \pm & 0.02 & 21.030 & 140 & \pm & 20 & 98.00 & 11.6 & \pm 0.5 & 15.600 & 155 & 2 \\
\hline 21.59 & \pm & 0.02 & 21.650 & 80 & \pm & 40 & 114.00 & 0.34 & \pm 0.08 & 0.376 & 157 & 2 \\
\hline 22.30 & \pm & 0.04 & 22.300 & 100 & \pm & 40 & 96.00 & 7.1 & \pm 0.8 & 6.000 & 158 & $3 / 2$ \\
\hline 22.5 & \pm & 0.2 & 22.330 & 100 & \pm 1 & .00 & 88.00 & 20 & 10 & 11.500 & 154 & $1 / 2$ \\
\hline 23.28 & \pm & 0.03 & 23.330 & 140 & \pm & 30 & 121.00 & 1.3 & 0.3 & 0.813 & 157 & 1 \\
\hline 23.60 & \pm & 0.02 & 23.670 & 140 & \pm & 10 & 120.00 & 2.91 & \pm 0.08 & 3.120 & 155 & 2 \\
\hline 25.35 & \pm & 0.01 & 25.400 & 30 & \pm & 30 & 85.00 & 1.99 & \pm 0.06 & 1.840 & 157 & 2 \\
\hline 27.509 & \pm & 0.002 & 27.570 & 140 & \pm & 20 & 125.00 & 1.31 & \pm 0.04 & 1.120 & 155 & 1 \\
\hline 29.50 & \pm & 0.02 & 29.580 & 113 & \pm & 2 & 108.00 & 4.8 & \pm 0.1 & 4.320 & 155 & 2 \\
\hline 30.05 & \pm & 0.02 & 30.100 & 130 & \pm & 10 & 100.00 & 11.1 & \pm 0.5 & 10.400 & 155 & 2 \\
\hline 31.66 & \pm & 0.01 & 31.720 & 140 & \pm & 20 & 118.00 & 1.24 & \pm 0.07 & 1.120 & 155 & 2 \\
\hline 33.1 & \pm & 0.2 & 33.140 & 110 & \pm & 30 & 109.80 & 1.6 & \pm 0.6 & 1.867 & 155 & 1 \\
\hline 33.14 & \pm & 0.03 & 33.230 & 98 & \pm & 3 & 90.00 & 14 & \pm & 14.600 & 156 & $1 / 2$ \\
\hline 33.4 & \pm & 0.3 & 33.510 & 120 & \pm & 90 & 115.00 & 1 & 4 & 1.600 & 155 & 1 \\
\hline 34.73 & \pm & 0.02 & 34.830 & 131 & \pm & 4 & 152.00 & 6.8 & 0.2 & 6.133 & 155 & 1 \\
\hline 35.39 & \pm & 0.01 & 35.470 & 140 & \pm & 10 & 118.00 & 2.17 & 0.06 & 1.840 & 155 & 2 \\
\hline 36.86 & & & 36.860 & 56 & & & 56.00 & 84 & & 84.000 & 152 & $1 / 2$ \\
\hline 37.066 & \pm & 0.003 & 37.120 & 139 & \pm & 6 & 101.00 & 8.3 & 0.3 & 8.400 & 155 & 1 \\
\hline 38.93 & \pm & 0.01 & 39.000 & 130 & \pm & 60 & 118.00 & 1.25 & \pm 0.07 & 1.040 & 155 & 2 \\
\hline 39.30 & & & 39.300 & 56 & & & 56.00 & 39 & & 39.000 & 152 & $1 / 2$ \\
\hline 40.08 & \pm & 0.01 & 40.170 & 120 & \pm & 40 & 110.00 & 1.6 & 0.2 & 1.307 & 157 & 1 \\
\hline 42.73 & & & 42.730 & 56 & & & 56.00 & 3.06 & & 3.060 & 152 & $1 / 2$ \\
\hline 43.83 & \pm & 0.07 & 43.920 & 140 & \pm & 90 & 136.00 & 18 & \pm & 17.333 & 155 & 1 \\
\hline 44.11 & \pm & 0.04 & 44.220 & 120 & \pm & 70 & 96.00 & 9 & \pm & 8.960 & 157 & 2 \\
\hline 45.98 & \pm & 0.02 & 46.100 & 128 & \pm & 6 & 126.00 & 2.3 & 0.1 & 2.240 & 155 & 2 \\
\hline 46.79 & \pm & 0.02 & 46.870 & 140 & \pm & 30 & 100.00 & 10.2 & 0.4 & 5.360 & 155 & 2 \\
\hline 47.18 & \pm & 0.04 & 47.070 & 89 & \pm & 8 & 88.00 & 2.4 & 0.6 & 3.200 & 154 & $1 / 2$ \\
\hline 47.628 & \pm & 0.006 & 47.730 & 107 & \pm & 10 & 109.80 & 0.39 & \pm 0.03 & 0.653 & 155 & 1 \\
\hline 48.68 & \pm & 0.03 & 48.790 & 118 & $\underline{I}$ & 9 & 90.00 & 26.7 & \pm 0.5 & 24.000 & 157 & 2 \\
\hline 49.63 & & 0.07 & 49.500 & 90 & \pm & 40 & 88.00 & 3 & 1 & 1.800 & 154 & $1 / 2$ \\
\hline 51.25 & \pm & 0.03 & 51.380 & 130 & \pm & 30 & 109.80 & 20.3 & 0.6 & 18.667 & 155 & 1 \\
\hline 52.01 & \pm & 0.03 & 52.130 & 140 & \pm & 20 & 115.00 & 20.9 & 0.8 & 19.467 & 155 & 1 \\
\hline
\end{tabular}


Table VII (continued) Epithermal Results: Resonance Parameters

\begin{tabular}{|c|c|c|c|c|c|c|c|c|c|c|c|c|c|}
\hline \multirow[b]{2}{*}{52.89} & \multicolumn{2}{|c|}{$\begin{array}{c}\text { Inergy } \\
\text { eV }\end{array}$} & \multirow[t]{2}{*}{$\begin{array}{c}\text { Energy } \\
\text { ENDF } \\
\text { meV } \\
53.030\end{array}$} & \multicolumn{3}{|c|}{$\begin{array}{c}\Gamma_{V} \\
\text { meV }\end{array}$} & \multirow[t]{2}{*}{$\begin{array}{c}\Gamma_{Y} \\
\text { ENDF } \\
\text { meV } \\
109.80\end{array}$} & \multicolumn{3}{|c|}{$\begin{array}{c}\Gamma_{\mathrm{n}} \\
\text { (2ag } \Gamma_{\mathrm{n}} \text { for } \\
\text { unassigned) } \\
\text { meV }\end{array}$} & \multirow[t]{2}{*}{$\begin{array}{c}\Gamma_{\mathrm{n}} \\
\text { ENDF } \\
\mathrm{meV} \\
1.360\end{array}$} & \multirow[t]{2}{*}{$\begin{array}{l}\text { ISO- } \\
\text { TOPE } \\
\text { ENDF } \\
155\end{array}$} & \multirow{2}{*}{$\begin{array}{c}\mathrm{J} \\
\text { ENDF } \\
2\end{array}$} \\
\hline & \pm & 0.02 & & 80 & \pm & 30 & & 1.2 & \pm & 0.2 & & & \\
\hline 53.62 & \pm & 0.02 & 53.740 & 140 & \pm & 30 & 92.00 & 8.7 & \pm & 0.2 & 7.680 & 155 & 2 \\
\hline 56.12 & \pm & 0.01 & 56.220 & 120 & \pm & 40 & 120.00 & 2.5 & \pm & 0.1 & 2.160 & 155 & 2 \\
\hline 58.26 & \pm & 0.03 & 58.300 & 140 & \pm & 20 & 101.00 & 32.0 & \pm & 0.6 & 28.000 & 157 & 2 \\
\hline 59.30 & \pm & 0.01 & 59.320 & 140 & \pm & 40 & 129.00 & 6.9 & \pm & 0.4 & 6.640 & 155 & 2 \\
\hline 62.73 & \pm & 0.02 & 62.840 & 150 & \pm & 30 & 90.00 & 8.5 & \pm & 0.5 & 8.000 & 155 & 2 \\
\hline 64.028 & \pm & 0.006 & 64.090 & 110 & \pm & 40 & 109.80 & 0.49 & \pm & 0.05 & 0.256 & 155 & 2 \\
\hline 65.21 & \pm & 0.01 & 65.060 & 100 & \pm & 20 & 57.00 & 32 & \pm & 5 & 24.000 & 154 & $1 / 2$ \\
\hline 66.4 & \pm & 0.5 & 65.200 & 120 & \pm & 10 & 109.80 & 0.5 & \pm & 0.4 & 1.333 & 155 & 1 \\
\hline 66.53 & \pm & 0.01 & 66.560 & 130 & \pm & 60 & 67.00 & 16 & \pm & 2 & 14.667 & 157 & 1 \\
\hline 69.4 & \pm & 0.1 & 69.400 & 100 & & 100 & 109.80 & 12 & \pm & 4 & 6.320 & 155 & 2 \\
\hline 74.34 & & & 74.340 & 50 & & & 50.40 & 60 & & & 60.000 & 152 & $1 / 2$ \\
\hline 76.00 & \pm & 0.03 & 76.120 & 90 & \pm & 50 & 88.00 & 2.0 & \pm & 0.9 & 1.100 & 154 & $1 / 2$ \\
\hline 76.85 & \pm & 0.01 & 77.000 & 110 & \pm & 60 & 109.80 & 3.0 & \pm & 0.3 & 1.600 & 155 & 2 \\
\hline 77.63 & \pm & 0.01 & 77.800 & 110 & \pm & 20 & 109.80 & 0.9 & \pm & 0.1 & 1.200 & 155 & 1 \\
\hline 78.75 & \pm & 0.06 & 78.800 & 110 & \pm & 30 & 109.80 & 8 & \pm & 1 & 4.240 & 155 & 2 \\
\hline 80.04 & \pm & 0.07 & 80.200 & 80 & \pm & 7 & 86.00 & 80 & \pm & 20 & 50.900 & 156 & $1 / 2$ \\
\hline 80 & \pm & 1 & 80.050 & 112 & \pm & 4 & 109.80 & 0 & \pm & 3 & 0.312 & 155 & 2 \\
\hline 80.9 & \pm & 0.3 & 80.900 & 110 & \pm & 30 & 109.80 & 1.44 & \pm & 0.09 & 2.400 & 155 & 1 \\
\hline 81.30 & \pm & 0.04 & 81.480 & 110 & \pm & 40 & 108.00 & 24 & \pm & 2 & 20.000 & 157 & 1 \\
\hline 82.10 & \pm & 0.04 & 82.240 & 100 & \pm & 70 & 99.95 & 7.1 & \pm & 0.6 & 6.160 & 157 & 2 \\
\hline 83.97 & \pm & 0.02 & 84.200 & 120 & \pm & 40 & 109.80 & 10.3 & \pm & 0.1 & 9.200 & 155 & 1 \\
\hline 84.91 & \pm & 0.01 & 85.000 & 110 & \pm & 40 & 109.80 & 2.2 & \pm & 0.3 & 3.067 & 155 & 1 \\
\hline 85.55 & & & 85.550 & 58 & & & 58.60 & 5.11 & & & 5.110 & 152 & $1 / 2$ \\
\hline 87.17 & \pm & 0.03 & 87.210 & 140 & \pm & 10 & 128.00 & 11.1 & \pm & 0.4 & 10.160 & 157 & 2 \\
\hline 90.51 & \pm & 0.02 & 90.500 & 110 & \pm & 90 & 109.80 & 2.5 & \pm & 0.2 & 1.280 & 155 & 2 \\
\hline 92.40 & & & 92.400 & 58 & & & 58.60 & 142 & & & 142.000 & 152 & $1 / 2$ \\
\hline 92.47 & \pm & 0.02 & 92.500 & 110 & \pm & 20 & 109.80 & 2.14 & \pm & 0.06 & 2.160 & 155 & 2 \\
\hline 92.90 & \pm & 0.03 & 92.800 & 110 & \pm & 50 & 109.80 & 3.48 & \pm & 0.07 & 3.120 & 155 & 2 \\
\hline 93.99 & \pm & 0.01 & 94.100 & 110 & \pm & 40 & 109.80 & 0.64 & \pm & 0.09 & 0.544 & 155 & 2 \\
\hline 95.70 & \pm & 0.03 & 95.700 & 110 & \pm & 50 & 109.80 & 7.1 & \pm & 0.4 & 3.840 & 155 & 2 \\
\hline 96.4 & \pm & 0.2 & 96.600 & 110 & \pm & 50 & 109.80 & 3.8 & \pm & 0.7 & 6.267 & 155 & 1 \\
\hline 96.6 & \pm & 0.1 & 96.520 & 100 & \pm & 40 & 110.00 & 22.0 & \pm & 0.4 & 12.160 & 157 & 2 \\
\hline 98.30 & \pm & 0.03 & 98.300 & 150 & \pm & 20 & 109.80 & 11.7 & \pm & 0.4 & 17.333 & 155 & 1 \\
\hline 99.9 & \pm & 0.1 & 100.200 & 110 & \pm & 10 & 109.80 & 2.5 & \pm & 0.2 & 2.133 & 155 & 1 \\
\hline 100.16 & \pm & 0.06 & 100.200 & 100 & \pm & 30 & 94.00 & 43 & \pm & 1 & 46.667 & 157 & 1 \\
\hline 100.72 & \pm & 0.08 & 100.700 & 90 & \pm & 40 & 82.00 & 48 & \pm & 7 & 32.000 & 154 & $3 / 2$ \\
\hline 101.20 & \pm & 0.09 & 101.100 & 120 & \pm & 10 & 105.00 & 1.3 & \pm & 0.2 & 1.000 & 158 & $1 / 2$ \\
\hline 101.42 & \pm & 0.02 & 101.400 & 140 & \pm & 30 & 109.80 & 2.1 & \pm & 0.2 & 2.720 & 155 & 2 \\
\hline 102.03 & \pm & 0.03 & 102.100 & 110 & \pm & 50 & 109.80 & 1.52 & \pm & 0.06 & 1.733 & 155 & 1 \\
\hline 104.36 & \pm & 0.09 & 104.400 & 110 & \pm & 80 & 109.80 & 5 & \pm & 1 & 9.067 & 155 & 1 \\
\hline 104.89 & \pm & 0.08 & 104.950 & 103 & \pm & 2 & 70.00 & 70 & \pm & 40 & 57.333 & 157 & 1 \\
\hline 105.8 & \pm & 0.1 & 105.900 & 140 & \pm & 20 & 109.80 & 6 & \pm & 1 & 6.133 & 155 & 1 \\
\hline 106.05 & \pm & 0.08 & 105.600 & 110 & \pm & 20 & 88.00 & 11 & \pm & 2 & 4.800 & 154 & $1 / 2$ \\
\hline 107.14 & \pm & 0.04 & 107.100 & 110 & \pm & 80 & 109.80 & 9 & \pm & 2 & 6.240 & 155 & 2 \\
\hline 107.46 & \pm & 0.06 & 107.460 & 120 & \pm & 30 & 99.95 & 4 & \pm & 1 & 5.600 & 157 & 2 \\
\hline 109.37 & \pm & 0.02 & 109.600 & 115 & \pm & 2 & 109.80 & 7.3 & \pm & 0.5 & 4.667 & 155 & 1 \\
\hline 110.54 & \pm & 0.07 & 110.460 & 140 & \pm & 50 & 85.00 & 50 & \pm & 20 & 42.400 & 157 & 2 \\
\hline 112.40 & \pm & 0.04 & 112.400 & 90 & \pm & 70 & 84.00 & 9.1 & \pm & 0.2 & 9.040 & 155 & 2 \\
\hline 113.81 & \pm & 0.05 & 113.800 & 130 & \pm & 20 & 67.00 & 20 & \pm & 1 & 15.200 & 155 & 2 \\
\hline 115.37 & \pm & 0.06 & 115.350 & 140 & \pm & 20 & 112.00 & 22.2 & \pm & 0.9 & 19.200 & 157 & 2 \\
\hline
\end{tabular}


Table VII (continued) Epithermal Results: Resonance Parameters

\begin{tabular}{|c|c|c|c|c|c|c|c|c|c|c|c|c|c|}
\hline \multirow[b]{2}{*}{116.56} & \multicolumn{2}{|c|}{$\begin{array}{c}\text { Energy } \\
\text { eV }\end{array}$} & \multirow[t]{2}{*}{$\begin{array}{c}\text { Energy } \\
\text { ENDF } \\
\text { meV } \\
116.500\end{array}$} & \multirow[b]{2}{*}{120} & \multicolumn{2}{|c|}{$\begin{array}{c}\Gamma_{V} \\
m e V\end{array}$} & \multirow[t]{2}{*}{$\begin{array}{c}\Gamma_{\mathrm{Y}} \\
\text { ENDF } \\
\text { meV } \\
116.00\end{array}$} & \multicolumn{3}{|c|}{$\begin{array}{c}\Gamma_{\mathrm{n}} \\
\left(2 \operatorname{agg} \Gamma_{\mathrm{n}} \text { for }\right. \\
\underset{\text { unassigned })}{\text { meV }}\end{array}$} & \multirow[t]{2}{*}{$\begin{array}{c}\Gamma_{\mathrm{n}} \\
\text { ENDF } \\
\text { meV } \\
17.333\end{array}$} & \multirow[t]{2}{*}{$\begin{array}{l}\text { ISO- } \\
\text { TOPE } \\
\text { ENDF } \\
155\end{array}$} & \multirow{2}{*}{$\begin{array}{l}J \\
\text { ENDF } \\
1\end{array}$} \\
\hline & \pm & 0.06 & & & \pm & 80 & & 21 & \pm & 1 & & & \\
\hline 118.66 & \pm & 0.02 & 118.600 & 110 & \pm & 50 & 109.80 & 2.5 & $\overline{ \pm}$ & 0.4 & 2.000 & 155 & 2 \\
\hline 120.83 & \pm & 0.01 & 121.000 & 130 & \pm & 30 & 91.00 & 140 & \pm & 40 & 132.000 & 157 & 2 \\
\hline 123.35 & \pm & 0.05 & 123.400 & 200 & & .00 & 159.00 & 40 & \pm & 6 & 36.000 & 155 & 1 \\
\hline 124.25 & \pm & 0.08 & 124.000 & 110 & \pm & 50 & 85.00 & 150 & \pm & 20 & 124.000 & 154 & $1 / 2$ \\
\hline 124.49 & \pm & 0.03 & 124.400 & 120 & \pm & 20 & 109.80 & 4 & \pm & 1 & 6.640 & 155 & 2 \\
\hline 126.11 & \pm & 0.02 & 126.000 & 110 & \pm & 60 & 109.80 & 14.6 & \pm & 0.4 & 20.533 & 155 & 1 \\
\hline 128.53 & \pm & 0.02 & 128.600 & 110 & \pm & 30 & 109.80 & 1.7 & \pm & 0.2 & 1.120 & 155 & 2 \\
\hline 129.82 & \pm & 0.01 & 129.800 & 110 & \pm & 40 & 109.80 & 3.4 & \pm & 0.3 & 2.560 & 155 & 2 \\
\hline 130.79 & \pm & 0.01 & 130.800 & 150 & \pm & 30 & 109.80 & 22 & \pm & 3 & 48.533 & 155 & 1 \\
\hline 131.37 & \pm & 0.01 & NEW & 130 & \pm & 10 & NEW & 1.27 & \pm & 0.08 & NEW & UNAS & IGNED \\
\hline 133.04 & \pm & 0.01 & 133.000 & 140 & \pm & 20 & 109.80 & 5.3 & \pm & 0.4 & 3.733 & 155 & 1 \\
\hline 133.95 & \pm & 0.01 & 133.800 & 110 & \pm & 30 & 109.80 & 3.4 & \pm & 0.2 & 2.320 & 155 & 2 \\
\hline 135.13 & \pm & 0.02 & 134.700 & 110 & \pm & 60 & 109.80 & 1.9 & \pm & 0.1 & 0.880 & 155 & 2 \\
\hline DISCAF & $\overrightarrow{D E}$ & & 135.100 & & & & 99.95 & & & & 0.880 & 157 & 2 \\
\hline DISCAF & DE & & 137.900 & & & & 99.95 & & & & 78.667 & 157 & 1 \\
\hline 137.99 & \pm & 0.08 & 137.800 & 120 & \pm & 80 & 109.80 & 90 & \pm & 30 & 21.333 & 155 & 1 \\
\hline 138.2 & \pm & 0.2 & 138.700 & 100 & \pm & 10 & 86.00 & 21 & \pm & 9 & 82.667 & 157 & 1 \\
\hline 138.9 & \pm & 0.2 & 139.200 & 94 & \pm & 8 & 91.00 & 40 & \pm & 10 & 124.000 & 154 & $3 / 2$ \\
\hline 139.37 & \pm & 0.05 & 139.300 & 100 & \pm & 70 & 99.95 & 40 & \pm & 10 & 10.000 & 157 & 1 \\
\hline 140.00 & & & 140.000 & 58 & & & 58.60 & 78.8 & & & 78.800 & 152 & $1 / 2$ \\
\hline 140.55 & \pm & 0.05 & 140.400 & 130 & \pm & 10 & 109.80 & 4.9 & \pm & 0.3 & 4.133 & 155 & 1 \\
\hline 141.30 & \pm & 0.01 & 141.400 & 120 & \pm & 10 & 109.80 & 1.69 & \pm & 0.08 & 1.040 & 155 & 2 \\
\hline 143.75 & \pm & 0.01 & 143.610 & 130 & \pm & 30 & 88.00 & 60 & \pm & 10 & 60.000 & 157 & 2 \\
\hline 145.66 & \pm & 0.01 & 145.600 & 150 & \pm & 20 & 109.80 & 6.5 & \pm & 0.3 & 6.160 & 155 & 2 \\
\hline 147.02 & \pm & 0.01 & 146.900 & 130 & \pm & 10 & 109.80 & 5.3 & \pm & 0.2 & 3.760 & 155 & 2 \\
\hline 148.2 & \pm & 0.2 & 148.400 & 120 & \pm & 20 & 88.00 & 46 & \pm & 10 & 38.000 & 154 & $1 / 2$ \\
\hline 148.4 & \pm & 0.3 & 148.200 & 110 & \pm & 10 & 109.80 & 8.6 & \pm & 0.9 & 9.600 & 155 & 2 \\
\hline 148.55 & \pm & 0.05 & 148.310 & 140 & \pm & 30 & 99.95 & 24 & \pm & 1 & 24.000 & 157 & 1 \\
\hline 149.53 & \pm & 0.03 & 149.600 & 110 & \pm & 40 & 109.80 & 36 & \pm & 2 & 33.333 & 155 & 1 \\
\hline 150.37 & \pm & 0.04 & 150.200 & 110 & \pm & 40 & 109.80 & 80 & \pm & 30 & 24.800 & 155 & 2 \\
\hline 150.62 & \pm & 0.03 & 151.200 & 80 & \pm & 30 & 86.00 & 23 & \pm & 7 & 41.700 & 156 & $1 / 2$ \\
\hline 152.27 & \pm & 0.01 & 152.200 & 150 & \pm & 40 & 109.80 & 6.2 & \pm & 0.8 & 8.000 & 155 & 1 \\
\hline 153.80 & \pm & 0.05 & 154.000 & 160 & \pm & 30 & 109.80 & 1.1 & \pm & 0.2 & 1.120 & 155 & 2 \\
\hline 156.4 & \pm & 0.1 & 156.300 & 110 & \pm & 80 & 109.80 & 30 & \pm & 10 & 7.680 & 155 & 2 \\
\hline 156.70 & \pm & 0.02 & 156.430 & 140 & \pm & 50 & 91.00 & 13 & \pm & 5 & 19.760 & 157 & 2 \\
\hline 160.00 & & & 160.000 & 58 & & & 58.60 & 2.83 & & & 2.830 & 152 & $1 / 2$ \\
\hline 160.03 & \pm & 0.07 & 160.100 & 110 & \pm & 50 & 109.80 & 10.3 & \pm & 0.5 & 9.600 & 155 & 2 \\
\hline 161.57 & \pm & 0.08 & 161.600 & 150 & \pm & 20 & 109.80 & 21.6 & \pm & 0.8 & 20.000 & 155 & 2 \\
\hline 164.8 & \pm & 0.2 & 164.500 & 98 & \pm & 7 & 77.00 & 158 & \pm & 2 & 105.000 & 154 & $3 / 2$ \\
\hline 165.00 & \pm & 0.09 & 164.830 & 100 & \pm & 80 & 100.00 & $23^{*}$ & \pm & 6 & 20.560 & 157 & 2 \\
\hline 168.20 & \pm & 0.09 & 168.300 & 123 & \pm & 6 & 109.80 & 31 & \pm & 4 & 30.133 & 155 & 1 \\
\hline 168.60 & \pm & 0.04 & 168.030 & 99. & 95 & & 99.95 & 3.33 & 3 & & 3.333 & 157 & 1 \\
\hline 169.4 & \pm & 0.1 & 169.250 & 90 & \pm & 10 & 99.95 & 3.4 & \pm & 0.2 & 3.280 & 157 & 2 \\
\hline 170.2 & \pm & 0.1 & 170.300 & 80 & \pm & 30 & 109.80 & 8 & \pm & 1 & 8.320 & 155 & 2 \\
\hline 170.4 & \pm & 0.1 & 170.400 & 85 & \pm & 9 & 88.00 & 4.9 & \pm & 0.4 & 5.000 & 154 & $1 / 2$ \\
\hline 171.2 & \pm & 0.2 & 171.250 & 100 & \pm & 10 & 99.95 & 120 & \pm & 40 & 44.000 & 157 & 1 \\
\hline 171.6 & \pm & 0.1 & 171.400 & 110 & \pm & 60 & 109.80 & 18 & \pm & 1 & 9.200 & 155 & 2 \\
\hline 173.5 & \pm & 0.1 & 173.500 & 110 & \pm & 80 & 109.80 & 33 & \pm & 2 & 32.800 & 155 & 2 \\
\hline 173.80 & & & 173.800 & 30 & & & 30.10 & 86 & & & 86.000 & 152 & $1 / 2$ \\
\hline 175.46 & \pm & 0.05 & 175.600 & 110 & \pm & 40 & 109.80 & 4.2 & \pm & 0.6 & 2.080 & 155 & 2 \\
\hline
\end{tabular}


Table VII (continued) Epithermal Results: Resonance Parameters

\begin{tabular}{|c|c|c|c|c|c|c|c|c|c|c|c|c|}
\hline \multirow[b]{2}{*}{177.99} & \multicolumn{2}{|c|}{$\begin{array}{c}\text { Energy } \\
\text { eV }\end{array}$} & \multirow[t]{2}{*}{$\begin{array}{c}\text { Energy } \\
\text { ENDF } \\
\text { meV } \\
178.000\end{array}$} & \multicolumn{2}{|r|}{$\begin{array}{c}\Gamma_{\mathrm{Y}} \\
\mathrm{meV}\end{array}$} & \multirow[t]{2}{*}{ 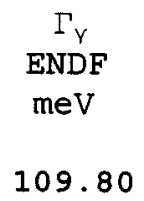 } & \multicolumn{3}{|c|}{$\begin{array}{c}\Gamma_{\mathrm{n}} \\
\left(2 \mathrm{ag} \Gamma_{\mathrm{n}} \text { for }\right. \\
\text { unassigned) } \\
\text { meV }\end{array}$} & \multirow{2}{*}{$\begin{array}{c}\Gamma_{\mathrm{n}} \\
\text { ENDF } \\
\text { meV } \\
9.733\end{array}$} & \multirow{2}{*}{$\begin{array}{l}\text { ISO- } \\
\text { TOPE } \\
\text { ENDF } \\
\\
155\end{array}$} & \multirow{2}{*}{$\begin{array}{l}\mathrm{J} \\
\text { ENDF } \\
1\end{array}$} \\
\hline & \pm & 0.02 & & 130 & \pm 10 & & 13 & \pm & 2 & & & \\
\hline 178.73 & \pm & 0.03 & 178.550 & 140 & \pm 20 & 145.00 & 17.0 & \pm & 1.0 & 16.000 & 157 & 2 \\
\hline 180.34 & \pm & 0.04 & 180.400 & 110 & \pm 40 & 109.80 & 9.7 & \pm & 0.3 & 14.667 & 155 & 1 \\
\hline 183.20 & \pm & 0.05 & NEW & 110 & \pm 40 & NEW & 1.3 & \pm & 0.2 & NEW & \multicolumn{2}{|c|}{ UNASSIGNED } \\
\hline 183.94 & \pm & 0.07 & 183.830 & 100 & \pm 90 & 99.95 & 34 & \pm & 8 & 17.600 & 157 & 2 \\
\hline 185.11 & \pm & 0.04 & NEW & 110 & \pm 60 & NEW & 0.6 & \pm & 0.1 & NEW & \multicolumn{2}{|c|}{ UNASSIGNED } \\
\hline 185.70 & & & 185.700 & 52 & & 52.50 & 84 & & & 84.000 & 152 & $3 / 2$ \\
\hline 187.36 & \pm & 0.07 & NEW & 100 & \pm 100 & NEW & 3.5 & \pm & 0.2 & NEW & \multicolumn{2}{|c|}{ UNASSIGNED } \\
\hline 189.30 & \pm & 0.06 & NEW & 100 & \pm 80 & NEW & 0.3 & \pm & 0.1 & NEW & \multicolumn{2}{|c|}{ UNASSIGNED } \\
\hline 190.9 & \pm & 0.1 & 190.730 & 100 & \pm 90 & 99.95 & 60 & \pm & 60 & 28.000 & 157 & 1 \\
\hline 194.6 & \pm & 0.1 & 194.530 & 110 & \pm 50 & 99.95 & 60 & \pm & 80 & 44.800 & 157 & 2 \\
\hline 198.4 & \pm & 0.2 & 198.100 & 92 & \pm 4 & 86.00 & 200 & \pm & 100 & 270.000 & 156 & $1 / 2$ \\
\hline 199.5 & \pm & 0.2 & 201.600 & 60 & \pm 40 & 88.00 & 80 & \pm & 50 & 11.700 & 154 & $3 / 2$ \\
\hline DISCAF & RE & & 201.600 & & & 86.00 & & & & 17.000 & 156 & $1 / 2$ \\
\hline 201.99 & \pm & 0.01 & 202.100 & 160 & \pm 40 & 86.00 & 50 & \pm & 10 & 266.000 & 156 & $3 / 2$ \\
\hline DISCAF & $\mathrm{DE}$ & & 202.740 & & & 99.95 & & & & 9.600 & 157 & 1 \\
\hline 203.10 & & & $203: 100$ & 58 & & 58.80 & 97 & & & 97.000 & 152 & $1 / 2$ \\
\hline 203.39 & \pm & 0.02 & NEW & 130 & \pm 10 & NEW & 0.98 & \pm & 0.04 & NEW & \multicolumn{2}{|c|}{ UNASSIGNED } \\
\hline 205.75 & \pm & 0.04 & 205.350 & 110 & \pm 10 & 99.95 & 2.0 & \pm & 0.1 & 0.976 & 157 & 2 \\
\hline DISCÁF & $D E$ & & 206.900 & & & 99.95 & & & & 1.360 & 157 & 2 \\
\hline DISCAF & $\mathrm{RE}$ & & 207.700 & & & 58.60 & & & & 5.230 & 152 & $1 / 2$ \\
\hline 207.77 & \pm & 0.04 & 207.810 & 150 & \pm 20 & 114.00 & 110 & \pm & 30 & 108.000 & 157 & 2 \\
\hline 209.1 & \pm & 0.2 & NEW & 120 & \pm 10 & NEW & 1.1 & \pm & 0.2 & NEW & \multicolumn{2}{|c|}{ UNASSIGNED } \\
\hline 210.32 & \pm & 0.01 & NEW & 140 & \pm 20 & NEW & 2.78 & \pm & 0.06 & NEW & \multicolumn{2}{|c|}{ UNASSIGNED } \\
\hline 211.57 & \pm & 0.02 & 211.000 & 99 & \pm 8 & 88.00 & 45 & \pm & 1 & 35.000 & 154 & $3 / 2$ \\
\hline 212.32 & \pm & 0.02 & NEW & 100 & \pm 10 & NEW & 0.64 & \pm & 0.02 & NEW & \multicolumn{2}{|c|}{ UNASSIGNED } \\
\hline 213.68 & \pm & 0.02 & NEW & 102 & \pm 10 & NEW & 1.02 & \pm & 0.03 & NEW & UNAS & 3 IGNED \\
\hline 214.77 & \pm & 0.01 & NEW & 130 & \pm 20 & NEW & 5.5 & \pm & 0.5 & NEW & UNAS & SIGNED \\
\hline 217.23 & \pm & 0.01 & 217.150 & 121 & \pm 9 & 99.95 & 19.9 & \pm & 0.9 & 8.000 & 157 & 1 \\
\hline 218.57 & \pm & 0.02 & NEW & 140 & \pm 10 & NEW & 1.89 & \pm & 0.03 & NEW & UNAS & IGNED \\
\hline 220.24 & \pm & 0.08 & 220.900 & 150 & \pm 20 & 99.95 & 8.3 & \pm & 0.4 & 4.000 & 157 & 1 \\
\hline 222.22 & \pm & 0.03 & 222.000 & 80 & \pm 20 & 120.00 & 50 & \pm & 20 & 50.000 & 160 & $1 / 2$ \\
\hline 223.30 & & & 223.300 & 64 & & 64.20 & 301 & & & 301.000 & 152 & 光 \\
\hline 224.90 & \pm & 0.02 & 224.000 & 100 & \pm 100 & 88.00 & 110 & \pm & 60 & 18.000 & 154 & $3 / 2$ \\
\hline 227.91 & \pm & 0.02 & 228.300 & 100 & \pm 100 & 99.95 & 52 & \pm & 3 & 6.560 & 157 & 2 \\
\hline 229.52 & \pm & 0.02 & NEW & 100 & \pm 70 & NEW & 9.7 & \pm & 0.5 & NEW & UNAS & 3 IGNED \\
\hline 230.86 & \pm & 0.05 & NEW & 100 & \pm 100 & NEW & 3.7 & \pm & 0.2 & NEW & UNAS & I IGNED \\
\hline 231.40 & & & 231.400 & 62 & & 62.00 & 46 & & & 46.000 & 152 & $1 / 2$ \\
\hline 232.85 & \pm & 0.01 & NEW & 100 & \pm 90 & NEW & 2.2 & \pm & 0.2 & NEW & UNA & IGNED \\
\hline 235.9 & \pm & 0.2 & NEW & 70 & \pm 60 & NEW & 1.4 & \pm & 0.2 & NEW & UNA & I IGNED \\
\hline 237.3 & \pm & 0.1 & NEW & 100 & \pm 100 & NEW & 5.8 & \pm & 0.2 & NEW & UNAS & SIGNED \\
\hline 238.00 & & & 238.000 & 100 & & 100.00 & 223.6 & & & 223.600 & 152 & $1 / 2$ \\
\hline 239.56 & \pm & 0.03 & 239.550 & 120 & \pm 20 & 99.95 & 250 & \pm & 40 & 152.000 & 157 & 2 \\
\hline 243.17 & \pm & 0.01 & 242.700 & 90 & \pm 20 & 105.00 & 50 & \pm & 20 & 60.000 & 158 & $1 / 2$ \\
\hline 245.16 & \pm & 0.02 & 244.000 & 98 & \pm 9 & 86.00 & 3.25 & \pm & 0.06 & 3.100 & 156 & $1 / 2$ \\
\hline 246.80 & \pm & 0.01 & 244.600 & 118 & \pm 9 & 99.95 & 19.8 & \pm & 0.5 & 4.400 & 157 & 1 \\
\hline 248.83 & \pm & 0.01 & 246.640 & 120 & \pm 10 & 99.95 & 5.0 & \pm & 0.1 & 9.280 & 157 & 2 \\
\hline 250.51 & \pm & 0.02 & 250.200 & 130 & \pm 10 & 99.95 & 8.2 & \pm & 0.2 & 5.733 & 157 & 1 \\
\hline 252.40 & & & 252.400 & 52 & & 52.40 & 127 & & & 127.000 & 152 & $1 / 2$ \\
\hline 253.25 & \pm & 0.03 & 252.800 & 101 & \pm 9 & 88.00 & 26 & \pm & 1 & 12.000 & 154 & $1 / 2$ \\
\hline 254.87 & \pm & 0.01 & 255.000 & 130 & \pm 10 & 99.95 & 18.6 & \pm & 0.5 & 3.600 & 157 & 1 \\
\hline
\end{tabular}


Table VII (continued) Epithermal Results: Resonance Parameters

\begin{tabular}{|c|c|c|c|c|c|c|c|c|c|c|c|}
\hline \multirow[b]{2}{*}{256.46} & \multicolumn{2}{|c|}{$\begin{array}{c}\text { inergy } \\
\text { ev }\end{array}$} & \multirow[t]{2}{*}{$\begin{array}{c}\text { Energy } \\
\text { ENDF } \\
\text { meV } \\
255.200\end{array}$} & \multicolumn{2}{|c|}{$\begin{array}{c}\Gamma_{Y} \\
m e V\end{array}$} & \multirow[t]{2}{*}{$\begin{array}{l}\Gamma_{Y} \\
\text { ENDF } \\
\text { meV } \\
99.95\end{array}$} & \multicolumn{2}{|c|}{$\begin{array}{c}\Gamma_{n} \\
\left(2 a g \Gamma_{n} \text { for }\right. \\
\text { unassigned) } \\
\text { meV }\end{array}$} & \multirow{2}{*}{$\begin{array}{c}\Gamma_{\mathrm{n}} \\
\text { ENDF } \\
\mathrm{meV} \\
3.733\end{array}$} & \multirow{2}{*}{$\begin{array}{l}\text { ISO- } \\
\text { TOPE } \\
\text { ENDF } \\
\\
157\end{array}$} & \multirow{2}{*}{$\begin{array}{l}\mathrm{J} \\
\text { ENDF } \\
1\end{array}$} \\
\hline & \pm & 0.06 & & 101 & \pm 10 & & 1.46 & \pm 0.09 & & & \\
\hline 258.01 & \pm & 0.01 & 257.500 & 91 & \pm 7 & 88.00 & 40 & $\pm \quad 1$ & 34.000 & 154 & $3 / 2$ \\
\hline 259.25 & \pm & 0.02 & NEW & 102 & \pm 10 & NEW & 1.11 & \pm 0.03 & NEW & UNASS & SIGNED \\
\hline 260.53 & \pm & 0.01 & 260.500 & 120 & \pm 10 & 99.95 & 31 & $\pm \quad 3$ & 21.867 & 157 & 1 \\
\hline 62.56 & \pm & 0.01 & NEW & 104 & \pm 10 & NEW & 0.96 & \pm 0.02 & NEW & UNASS & SIGNED \\
\hline 64.89 & \pm & 0.01 & NEW & 110 & \pm 10 & NEW & 1.30 & \pm 0.03 & NEW & UNASS & SIGNED \\
\hline 266.05 & \pm & 0.01 & 265.610 & 110 & \pm 10 & 99.95 & 7.9 & 0.2 & 6.400 & 157 & 2 \\
\hline 268.47 & \pm & 0.01 & 268.020 & 140 & \pm 20 & 99.95 & 17.0 & 0.9 & 10.480 & 157 & 2 \\
\hline 269.57 & \pm & 0.03 & 269.200 & 120 & \pm 20 & 88.00 & 50 & 10 & 28.000 & 154 & $3 / 2$ \\
\hline 272.36 & \pm & 0.02 & NEW & 100 & \pm 60 & NEW & 1.3 & 0.1 & NEW & UNASS & SIGNED \\
\hline 277.38 & \pm & 0.06 & 277.200 & 100 & \pm 300 & 105.00 & 40 & 60 & 18.000 & 158 & $1 / 2$ \\
\hline 279.40 & \pm & 0.03 & NEW & 98 & \pm 10 & NEW & 0.44 & \pm 0.03 & NEW & UNASS & SIGNED \\
\hline 282.60 & & & 282.600 & 49 & & 49.10 & 145 & & 145.000 & 152 & $1 / 2$ \\
\hline 282.28 & \pm & 0.05 & 281.640 & 110 & \pm 100 & 99.95 & 70 & 30 & 38.400 & 157 & 2 \\
\hline 284.2 & \pm & 1 & NEW & 100 & \pm 30 & NEW & 2.1 & 0.2 & NEW & UNASS & SIGNED \\
\hline 285.24 & \pm & 0.05 & NEW & 150 & \pm 40 & NEW & 2.7 & 0.4 & NEW & UNASS & SIGNED \\
\hline 287.89 & \pm & 0.04 & 287.330 & 100 & \pm 50 & 99.95 & 25 & 3 & 14.240 & 157 & 2 \\
\hline 288.99 & \pm & 0.03 & NEW & 140 & \pm 30 & NEW & 2.3 & 0.3 & NEW & UNASS & SIGNED \\
\hline 91.08 & \pm & 0.03 & 290.770 & 100 & \pm 50 & 99.95 & 51 & 9 & 65.333 & 157 & 1 \\
\hline 292.37 & \pm & 0.07 & NEW & 130 & \pm 30 & NEW & 2.2 & 0.2 & NEW & UNASS & SIGNED \\
\hline 293.40 & & & 293.400 & 71 & & 71.00 & 352 & & 352.000 & 152 & $1 / 2$ \\
\hline 94.16 & \pm & .01 & 293.700 & 130 & \pm 30 & 99.95 & 49 & \pm & 36.800 & 157 & 2 \\
\hline 295.79 & \pm & 0.08 & NEW & .00 & \pm 10 & NEW & 0.5 & 0.2 & NEW & UNASS & SIGNED \\
\hline 298.0 & \pm & 0.1 & NEW & 110 & \pm 10 & NEW & 0.38 & \pm 0.08 & NEW & UNASS & SIGNED \\
\hline
\end{tabular}

were not included in ENDF. Six resonances present in ENDF have been discarded because the literature does not demonstrate their existence nor do the present measurements support their existence. Parameters for several resonances, particularly those from ${ }^{152} \mathrm{Gd}$, were not fitted because the resonances were very weak. In these cases the resonances parameters are assigned ENDF values and are listed in Table VII without any quoted errors.

\section{VI.B.1 Results- Epithermal; The 1-10 eV region}

Results of the SAMMY fit to the $1-10 \mathrm{eV}$ region using thermal transmission and epithermal capture data are plotted in Figure 17 and Figure 18. The scatter of data at $1.4 \mathrm{eV}$ in Figure 17 is due to the fixed indium notch. The resulting resonance parameters are given in Table VII. The same three samples were measured in both transmission and capture, i.e., $0.025 \mathrm{~mm}(1 \mathrm{mil}), 0.051$ $\mathrm{mm}(2 \mathrm{mil})$, and $0.127 \mathrm{~mm}(5 \mathrm{mil})$ natural metal $\mathrm{Gd}$. The radiation width for the weak $3.6 \mathrm{eV}$ resonance in ${ }^{155} \mathrm{Gd}$ was not varied.

\section{VI.B.2 Results- Epithermal; The 10-300 eV region}

Results of the SAMMY fit from 10-300 eV are shown in Figure 19-Figure 54. Fifteen data sets were used in the region above $10 \mathrm{eV}$. There were two separate transmission measurements. 
The first used a $\mathrm{B}_{4} \mathrm{C}$ overlap filter and included samples of the following thicknesses $0.127 \mathrm{~mm}$ ( $5 \mathrm{mil}$ ), $0.254 \mathrm{~mm}$ (10 mil), $0.508 \mathrm{~mm}$ (20 mil), $0.889 \mathrm{~mm}$ (35 mil), and 1.27 $\mathrm{mm}$ (50 mil). The second epithermal transmission measurement used a Cd overlap filter and included samples of the following thicknesses: $0.508 \mathrm{~mm}$ (20 mil), $0.889 \mathrm{~mm}$ (35 $\mathrm{mil}), 1.27 \mathrm{~mm}$ (50 mil), $2.54 \mathrm{~mm}$ (100 mil), $5.08 \mathrm{~mm}$ (200 mil), and $1.02 \mathrm{~cm}$ (400 mil). The capture measurement used a $\mathrm{Cd}$ overlap filter and included samples of the following thicknesses: $0.025 \mathrm{~mm}$ (1 mil), $0.051 \mathrm{~mm}$ ( $2 \mathrm{mil}), 0.127 \mathrm{~mm}(5 \mathrm{mil})$, and $2.54 \mathrm{~mm}$ (100 mil).

The resolved resonance energy region for ${ }^{155} \mathrm{Gd}$ in the ENDF/B-VI evaluation ends at $180 \mathrm{eV}$. As a result, fitting data above $180 \mathrm{eV}$ was performed without initial estimates for resonance locations and widths, a challenging task.

Finally, if a resonance is clearly observed in both transmission and capture it has been added to the database shown in Table VII. In these cases, the isotope and spin are listed as unassigned and their associated neutron widths are given in Table VII as $2 \mathrm{ag} \Gamma_{\mathbf{n}}$ where ' $a$ ' is abundance and ' $g$ ' is the statistical weighting factor, $g=(2 J+1) /[2(2 \mathrm{I}+1)]$, where $I$ is the spin of the target nucleus and $J$ is the total angular momentum of the compound state (also known as the spin state of the resonance) in units of $h / 2 \pi$ where $h$ is Plank's constant. A more detailed discussion of the challenges and resolutions in the epithermal region is presented in subsections VI.B.2.1 - VI.B.2.9.

\section{VI.B.2 Results- Epithermal; The $10-78 \mathrm{eV}$ region}

An overview of the region is given in Figure 19 and Figure 20 for transmission and capture, respectively. The corresponding resonance parameters are given in Table VII. A challenging region to fit occurred at $33 \mathrm{eV}$.

The $33 \mathrm{eV}$ region fit is shown in Figure 21 and Figure 22 for transmission and capture, respectively. There is an energy mismatch between transmission (Figure 21) and capture data (Figure 22) due to independent measurements of flight path and zero time. The offset is $\approx 0.03 \mathrm{eV}$, (see $\Delta \mathrm{E}_{0}$ in Table VI for the $33.14 \mathrm{eV}$ resonance). The offset does not affect the SAMMY fit. The resonances at $33 \mathrm{eV}$ are identified in ENDF as a triplet. The 33.0-33.9 eV region is also identified as a triplet in Reference 29. According to these references, there are two ${ }^{155} \mathrm{Gd}$ resonances and one ${ }^{156} \mathrm{Gd}$ resonance in the triplet. Two authors ${ }^{12,17}$ saw both ${ }^{155} \mathrm{Gd}$ resonances and two authors ${ }^{10,30}$ saw the ${ }^{156} \mathrm{Gd}$ resonance. The triplet is unresolved in the current natural metal data. There is not enough information in the current data to precisely specify the resonance parameters of all three component resonances. Only the overall width of the triplet can be determined accurately. Therefore the uncertainties on the resonance parameters for the two small component resonances, $33.1 \mathrm{eV}$ and $33.4 \mathrm{eV}$ in ${ }^{155} \mathrm{Gd}$, are rather large. 


\section{VI.B.2.2 Results- Epithermal; The 78-120 eV region}

An overview of the region is given in Figure 23 and Figure 24. The corresponding resonance parameters are given in Table VII. Technical challenges in this region occur at 80 and $96 \mathrm{eV}$.

The resonances in the 78-80 eV region are small and their energies were constrained within SAMMY to stay near ENDF energies because the unconstrained calculation was found to move the resonances. That is, SAMMY did not see sufficient evidence that these resonances existed at the ENDF energies. But, at their unconstrainedfit energies, the resonances remained small and insignificant, implying that there is no compelling evidence in these data of their existence elsewhere either. Therefore, uncertainties in resonance parameters in this region are large.

There is a Cd resonance at $89 \mathrm{eV}$ which introduces some scatter in the data (Figure 24).

The 94-98 eV region shown in Figure 25 and Figure 26 was also difficult to fit. In addition to the resonance at $95.7 \mathrm{eV}$, there are two resonances (at least) at $96.5 \mathrm{eV}$. The $96.5 \mathrm{eV}$ region was treated as a doublet, and it fits the transmission data, but not the capture data. Below $95.5 \mathrm{eV}$ the calculation matches the capture data, but not the transmission data.

Many radiation widths, if left unconstrained, would be fit to unlikely large values. These radiation widths were subsequently constrained. And their uncertainties in Table VII reflect the possibility that the unconstrained values may be correct. A discussion of uncertainties in the epithermal region is given in Section VII.B.

\section{VI.B.2.3 Results- Epithermal; The 120-160 eV region}

Resonances in this region are shown in Figure 27 and Figure 28. The initial calculation using ENDF resonance parameters is reasonably close to the current data.

The SAMMY fit in the $120 \mathrm{eV}$ region is a further illustration of fitting difficulties. ENDF gives a single resonance at $120 \mathrm{eV}$. The fit to a single resonance is shown in Figure 29. There appears to be structure at $120.0 \mathrm{eV}$. A small 'shoulder' resonance was added to the fit at $120.0 \mathrm{eV}$, but the fit is still poor (see Figure 30). A two-step procedure was tried where first, the high-energy side of the resonance only was fitted (120.8-122.0 $\mathrm{eV}$ ), then the main resonance was fixed and the 'shoulder' was added. The fit did not improve. The doublet fits were insufficient to justify including an additional resonance in the final set of resonance parameters. The singlet fit shown in Figure 29 represents the final resonance parameters. A level density analysis (Section VI.B.2.9) shows a large probability of a multiplet. 
There are two large peaks in the data at $123 \mathrm{eV}$ and $124 \mathrm{eV}$ shown in Figure 31 and Figure 32. The neutron width fitted to the $123.35 \mathrm{eV}$ resonance is close to the ENDF value. The peak at approximately $124 \mathrm{eV}$ is a doublet whose resonance parameters produce an improved fit to transmission capture data when compared to ENDF.

Initial fits to the 129-132 $\mathrm{eV}$ region were of rather poor quality. A resonance was added at $131 \mathrm{eV}$ and, as a result, the entire 129-132 eV region fits very well, see Figure 31 and Figure 32. The shape of the ENDF curve and the bulge on the high-energy side of the large $130.79 \mathrm{eV}$ resonance demonstrate that a new resonance was needed.

Data in energy range 134-142 eV are shown in Figure 33 and Figure 34. ENDF identifies two resonances near $135 \mathrm{eV}$, one at $134.7 \mathrm{eV}$ in ${ }^{155} \mathrm{Gd}$ and the other at $135.1 \mathrm{eV}$ in ${ }^{157} \mathrm{Gd}$. Curiously, both resonances have exactly the same neutron width in ENDF. And an examination of the literature shows that no author saw both resonances. Fricke et al. ${ }^{12}$ saw a resonance at $134.8 \mathrm{eV}$ in ${ }^{155} \mathrm{Gd}$. But the same reference cites no resonance near $135 \mathrm{eV}$ in ${ }^{157} \mathrm{Gd}$. Also, Mughabghab and Chrien ${ }^{10}$ did not see a ${ }^{157} \mathrm{Gd}$ resonance near $135 \mathrm{eV}$. Therefore, no $135 \mathrm{eV}$ resonance in ${ }^{157} \mathrm{Gd}$ was included in the present fit.

A review of the literature casts some doubt on the uniqueness of the three ${ }^{157} \mathrm{Gd}$ resonances given by ENDF at 137.9, 138.7, and $139.3 \mathrm{eV}$, all with $\mathrm{J}=1$. Fricke et al. ${ }^{12}$ saw only two resonances, one resonance at $137.8 \pm 0.2 \mathrm{eV}$ and one at $139.3 \pm 0.2 \mathrm{eV}$. He made no spin assignments. Mughabghab and Chrien ${ }^{10}$ saw only one resonance, at 138.9 $\pm 1.5 \mathrm{eV}$, with no spin assignment. So, it's quite likely that there are only two resonances in ${ }^{157} \mathrm{Gd}$ in the 137.9-139.3 eV range and the third one given in ENDF is extraneous. Therefore, only two resonances were used in the present fit. The ENDF assignments of $\mathrm{J}=1$ for both resonances were retained in the current fit. Mughabghab ${ }^{29}$ assigned $\mathrm{J}=2$ to the two of three resonances to which he assigns spin.

The ${ }^{152} \mathrm{Gd}$ resonance given in ENDF at $140 \mathrm{eV}$ has not been varied in the current fit. The reference for that resonance is not clear. It was not seen by Macklin ${ }^{15}$ who measured that energy region. Anufriev ${ }^{14}$ saw this resonance at $138 \mathrm{eV}$.

Three resonances at $148 \mathrm{eV}$ are cited by ENDF. Each of the three resonances, at $148.2 \mathrm{eV}$ in ${ }^{155} \mathrm{Gd}$, at $148.4 \mathrm{eV}$ in ${ }^{154} \mathrm{Gd}$, and at $148.31 \mathrm{eV}$ in ${ }^{157} \mathrm{Gd}$ was seen independently by three different authors. All three resonances have been included in the current fit.

\section{VI.B.2.4 Results- Epithermal; The $160-200 \mathrm{eV}$ region}

The fits to this energy region are shown in Figure 35 and Figure 36 . The most interesting areas in this energy range are at $165,168,170,171,173$, and $185-\mathrm{eV}$, and perhaps some interest in the $198-199 \mathrm{eV}$ region. For resonances not discussed in this section, Table VII shows they have been characterized similarly to ENDF. As usual, ${ }^{152} \mathrm{Gd}$ resonance energies and parameters were not varied. 
The first region of interest is $164.5-168.6 \mathrm{eV}$ (Figure 37 and Figure 38). ENDF and previous measurements designate one ${ }^{154} \mathrm{Gd}$ resonance at $164.5 \mathrm{eV}$ and one ${ }^{157} \mathrm{Gd}$ resonance at $164.83 \mathrm{eV}$ The present measurement did not resolve this doublet. Therefore, the shared area between the two resonances could not be definitively determined without separated isotopes.

The next two ENDF resonances occur at 168.03 and $168.3 \mathrm{eV}$. Fricke et al. ${ }^{12}$ saw these two resonances at the same energy. They saw the smaller one in ${ }^{157} \mathrm{Gd}$ and the larger one in ${ }^{155} \mathrm{Gd}$. Karzhavini et al. ${ }^{177}$ saw the ${ }^{155} \mathrm{Gd}$ resonance at lower energy, but only by $0.1 \mathrm{eV}$. Belyaev et al. ${ }^{16}$ saw the ${ }^{157} \mathrm{Gd}$ resonance at lower energy and assigned it $\mathrm{J}=2$, contrary to ENDF. Mughabghab and Chrien ${ }^{10}$ also have the larger resonance in ${ }^{155} \mathrm{Gd}$ at $168.3 \mathrm{eV}$. This doublet is not resolved in the present data. In the SAMMY fit, the widths of the ${ }^{157} \mathrm{Gd}$ resonance were fixed to ENDF values, and only the resonance energy was varied. The spin assignment for the ${ }^{157} \mathrm{Gd}$ resonance was $\mathrm{J}=1$, although the reference for that spin assignment was not found and could not be determined from the present data. The parameters of the ${ }^{155} \mathrm{Gd}$ resonance were varied.

The second region of interest is the $170-174 \mathrm{eV}$ region. As shown in Table VII, there are 6 resonances identified by ENDF in this energy range. Figure 39 and Figure 40 show data and the SAMMY fits in this region. ENDF specifies a doublet at 170.3-170.4 $\mathrm{eV}$ and a second doublet at 171.25-171.4. A literature review confirmed that all four resonances have been distinctly observed in data taken on separated isotopes (Fricke et al. ${ }^{12}$, Mughabghab and Chrien ${ }^{10}$, Karzhavini et al. ${ }^{17,18}$, and Belyaev et al..$^{16}$ ). The SAMMY fit to the current natural metal data resulted in each doublet's resonance energies being separated somewhat. The parameters of the lower $\approx 170.3 \mathrm{eV}$ doublet remained close to ENDF while the widths of the $\approx 171.4 \mathrm{eV}$ doublet are much larger than those of ENDF. Figure 39 and Figure 40 show the increased area (beyond ENDF specifications) of these resonances required to fit the current data. The two remaining resonances in this region occur at $173.5 \mathrm{eV}$ in ${ }^{155} \mathrm{Gd}$ and $173.8 \mathrm{eV}$ in ${ }^{152} \mathrm{Gd}$. These resonances are seen as an unresolved doublet in the current data. The parameters of the ${ }^{152} \mathrm{Gd}$ resonance were fixed to ENDF values, while the ${ }^{155} \mathrm{Gd}$ resonance parameters were fitted.

The third region of interest is $183-188 \mathrm{eV}$. ENDF specifies only two resonances in this region, a rather large resonance at $183.83 \mathrm{eV}$ in ${ }^{157} \mathrm{Gd}$ and a resonance at $185.7 \mathrm{eV}$ in ${ }^{152} \mathrm{Gd}$. Figure 41 and Figure 42 show a broad peak at $183.9 \mathrm{eV}$, a second peak at approximately $185 \mathrm{eV}$ and a third peak at approximately $187.4 \mathrm{eV}$. The $183-184 \mathrm{eV}$ shape could not be fitted with a single resonance. Therefore, a new resonance was proposed at $183.2 \mathrm{eV}$. SAMMY then fitted that doublet, assigning the stronger ${ }^{157} \mathrm{Gd}$ resonance at $183.94 \mathrm{eV}$ and the weaker resonance at $183.2 \mathrm{eV}$.

The $185.7 \mathrm{eV}$ resonance in ${ }^{152} \mathrm{Gd}$ is visible in the ENDF calculations in Figure 41 and Figure 42. The data suggest a resonance which is much larger. Since the abundance of ${ }^{152} \mathrm{Gd}$ in a natural metal sample is so small, $0.1 \%$, and the ENDF resonance energy not aligned with the peak in the data, a new resonance was proposed at $185.1 \mathrm{eV}$. The resulting fit shown in Figure 41 and Figure 42 is quite good in this region. To 
demonstrate the need for this resonance, fits without the additional resonance are shown in Figure 43 and Figure 44.

\section{VI.B.2.5 Results- Epithermal; The 199-222 eV region}

Resonances in this region are shown in Figure 45 and Figure 46. Four resonances from ENDF have been excluded from the present analysis; $201.6 \mathrm{eV}$ in ${ }^{156} \mathrm{Gd}, 202.74 \mathrm{eV}$ in ${ }^{157} \mathrm{Gd}, 206.9 \mathrm{eV}$ in ${ }^{157} \mathrm{Gd}$, and $207.7 \mathrm{eV}$ in ${ }^{152} \mathrm{Gd}$. There are two reasons for these omissions. First, no author has explicitly seen any of these four resonances. Second, inclusion of these resonances does not improve the fit to the data.

There are four resonances listed in ENDF in the $201-203 \mathrm{eV}$ region. The first of these, at $201.6 \mathrm{eV}$ in ${ }^{154} \mathrm{Gd}$ was identified by Reference 13. It has been moved to 199.4 $\mathrm{eV}$ in the present fit. The next two resonances, $201.6 \mathrm{eV}$ in ${ }^{156} \mathrm{Gd}$ and $202.1 \mathrm{eV}$ in ${ }^{156} \mathrm{Gd}$, have never both been observed. Mughabghab and Chrien ${ }^{10}$ and Coceva and Stefanon ${ }^{30}$ each saw a single resonance at 202.1 and $201.8 \mathrm{eV}$, respectively. However, two resonances are listed in Reference 29 and in ENDF. Only one resonance is listed at 202 $\mathrm{eV}$ in ${ }^{156} \mathrm{Gd}$ in the present analysis. Another specific omission of a resonance listed in ENDF occurred at $206.9 \mathrm{eV}$ in ${ }^{157} \mathrm{Gd}$. This resonance is small in ENDF, $\Gamma_{\mathrm{n}}=1.36 \mathrm{meV}$, and cannot be traced to any of the Gd experiments discussed in this report. It does not improve the fit and has been omitted from the present results. ENDF contains a ${ }^{152} \mathrm{Gd}$ resonance at $207.7 \mathrm{eV}$, but a review of the literature failed to reveal the source of this resonance. While, in general, ${ }^{152} \mathrm{Gd}$ resonances were not varied, this particular resonance was omitted from the present analysis. The effect of removing this resonance on the widths of the nearest resonance at $207.8 \mathrm{eV}$ is negligible.

Seven new resonances have been added in the $199-222 \mathrm{eV}$ region. The first, at $203.1 \mathrm{eV}$, is a shoulder on the $202 \mathrm{eV}$ resonance in ${ }^{156} \mathrm{Gd}$ (see Figure 45 and Figure 46). A new resonance has been proposed at $209.1 \mathrm{eV}$. It is a shoulder on the larger $207.77 \mathrm{eV}$ resonance in ${ }^{157} \mathrm{Gd}$. Five more resonances have been assigned at $210.32,212.32,213.68$, 214.77 , and $218.57 \mathrm{eV}$ to account for structure apparent in the data in Figure 45 and Figure 46.

\section{VI.B.2.6 Results- Epithermal; The $220-240 \mathrm{eV}$ region}

Resonances in this region are shown in Figure 47 and Figure 48. New resonances were introduced at $229.52,230.86,232.85,235.9$, and $237.3 \mathrm{eV}$. This is the least number of resonances that resulted in a reasonable fit to the data. As the fits to the data in the figures show, more resonances may be needed. A discussion of the justification of new resonances is given in Section VI.B.2.9. 


\section{VI.B.2.7 Results- Epithermal; The $240-270 \mathrm{eV}$ region}

Resonances in this region are shown in Figure 49 and Figure 50. New resonances were introduced at $259.25,262.56$, and $264.89 \mathrm{eV}$. These resonances are needed to fit the data. The fit to the capture data is particularly good.

\section{VI.B.2.8 Results- Epithermal; The 270-300 eV region}

Resonances in this region are shown in Figure 51 and Figure 52. Several resonances were added to the ENDF database in this region. The first, a resonance at $272.36 \mathrm{eV}$ is obviously needed to fit the data in the figures. Next is a resonance or resonances at $277 \mathrm{eV}$. This energy region has been fitted with one resonance in the present analysis. However, as shown in the figures, the fit could be improved. A second resonance at $277 \mathrm{eV}$ was postulated, and a superior fit was obtained, as shown in Figure 53 and Figure 54. This is an illustration of the situation in the region above approximately $150 \mathrm{eV}$. The fits could be improved with the addition of more resonances. However, in this case, the shape of the data shown in Figure 51 and Figure 52 is not compelling enough to suggest an additional resonance.

The next large resonance or doublet occurs at $282 \mathrm{eV}$. This energy region has been fitted with one resonance in the present analysis. This is a good example of another common occurrence at resonance energies above $150 \mathrm{eV}$. The resonance is strong in both the capture and total cross section measurements. A single resonance appears insufficient to fit the capture peaks in Figure 52 . The singlet and doublet fit at $282 \mathrm{eV}$ is shown in Figure 53 and Figure 54. However, in this case as well, the shape of the data shown in Figure 51 and Figure 52 is not compelling enough to suggest an additional resonance.

At $285 \mathrm{eV}$, a doublet was judged to be needed due to the shape of the data. Therefore, a doublet, both new resonances, is used in the final fit shown in Figure 51 and Figure 52.

One resonance appears in ENDF at $287.33 \mathrm{eV}$. The data show a doublet at 288 and $289 \mathrm{eV}$ (see Figure 51 and Figure 52), and a resonance was added there. One resonance appears in ENDF at $290.77 \mathrm{eV}$. The.data show a doublet at 291 and $292 \mathrm{eV}$ and a resonance was added there. One resonance appears in ENDF at $293.4 \mathrm{eV}$. There is clearly a high energy shoulder on this resonance which is especially visible in the capture data (Figure 52). Therefore, a resonance was added at $295.79 \mathrm{eV}$. Also, at $298 \mathrm{eV}$, a resonance is visible, particularly in capture, and has been added to the database.

\section{VI.B.2.9 Results- Epithermal; Justification for New Resonances}

Figure 55 shows a 'staircase' plot of gadolinium level density including all new resonances added during the present analysis. The plot of observed levels vs energy 
shows a good fit to a straight line which agrees with the statistical model of the nucleus up to about $50 \mathrm{eV}$. All levels are s-wave. Elemental gadolinium is shown because there is no assignment of isotope to the proposed new resonances. Above $50 \mathrm{eV}$ a significant number of levels are missed. Therefore, even with the resonances added in the present analysis, the expectation of constant level density vs energy is not exceeded.

\section{VI.C Results- Resonance Integrals and Thermal Cross Sections}

Thermal cross sections and infinitely dilute capture resonance integrals (RI) have been calculated using ENDF and RPI resonance parameters. The isotopic ENDF evaluations used are listed in Table VIII. These files were processed using NJOY ${ }^{28}$ into pointwise (Energy, cross section) data and isotopic thermal $(2200 \mathrm{~m} / \mathrm{s})$ cross sections were obtained. The original ENDF files were then modified by replacing the original File 2 resonance parameters with those determined in the present work for all resonances below $300 \mathrm{eV}$. All resonances listed in Table VI and Table VII, except those designated "UNASSIGNED" in those tables, were included. For each isotope the number of levels and the energy region affected are shown in Table VIII. The resulting modified-ENDF files were processed using $\mathrm{NJOY}^{28}$ and thermal cross sections and resonance integrals were obtained.

Thermal cross sections from the present measurements are compared to those of ENDF in Table IX and Table X. The units of all cross sections in the tables are barns. The units of abundance are percent. The most significant departure of the present results from ENDF thermal capture cross sections is in ${ }^{157} \mathrm{Gd}$. This $11 \%$ reduction (from 254000 barns for ENDF to 226000 barns for RPI) is consistent with the $\approx 9 \%$ reduction in neutron width for the thermal ${ }^{157} \mathrm{Gd}$ resonance (see Table V). An insignificant reduction in thermal capture cross section is seen in ${ }^{155} \mathrm{Gd}$. This is due to the competing effects of a $7 \%$ reduction in neutron width compensated by a $3.7 \%$ reduction in total width $\left(\Gamma_{\mathrm{n}}+\Gamma_{\gamma}\right)$ and an energy shift toward the thermal energy $(0.0253 \mathrm{eV})$ point. The thermal capture cross section of elemental gadolinium is $\approx 9 \%$ lower than that calculated from ENDF parameters.

A significant reduction of thermal elastic cross section (Table $\mathrm{X}$ ) of the present results from ENDF occurs in ${ }^{157} \mathrm{Gd}$ (from 1010 to 798 barns). Thermal elastic scattering cross sections are proportional to $\Gamma_{\mathrm{n}}{ }^{2}$. So, the reduction in thermal elastic cross section of ${ }^{157} \mathrm{Gd}$ is consistent with the $\approx 9 \%$ reduction in neutron width for the thermal ${ }^{157} \mathrm{Gd}$ resonance (see Table VI). Thermal elastic scattering cross section for ${ }^{157} \mathrm{Gd}$ has a large uncertainty since it is essentially the small difference of two large numbers (total and capture cross sections). ${ }^{156} \mathrm{Gd}$ also exhibits a large deviation from ENDF in its small and statistically uncertain thermal elastic cross section. ${ }^{156} \mathrm{Gd}$ has only 2 resonances below $100 \mathrm{eV}$. The increase in its thermal elastic cross section is due to the substantial increase in the neutron width of the $80 \mathrm{eV}$ resonance (see Table VII). However, the uncertainty on that neutron width (see Table VII) encompasses the majority of the increase. 
Table VIII - Details of the ENDF/B-VI evaluations used in the calculations of thermal cross sections and resonance integrals. The units of abundance are percent. The rightmost column indicates the upper energy of the resolved resonance region in the evaluation.

\begin{tabular}{|c|c|c|c|c|c|}
\hline \multicolumn{2}{|c|}{ ENDF and RPI } & \multicolumn{2}{|c|}{ ENDF/B-VI Gd Evaluations } & \multicolumn{2}{c|}{ RPI } \\
\hline Isotope & abund & Evaluator, Date & $\begin{array}{c}\text { Resolved } \\
\text { Resonance } \\
\text { Region }\end{array}$ & $\begin{array}{c}\# \\
\text { levels }\end{array}$ & $\begin{array}{c}\text { Energy } \\
\text { Range }\end{array}$ \\
\hline Gd152 & 0.20 & $\begin{array}{c}\text { Wright, 1994; Rev. } \\
2001\end{array}$ & $<2660 \mathrm{eV}$ & 0 & -- \\
\hline Gd154 & 2.18 & $\begin{array}{c}\text { Wright, 1994; Rev. } \\
2001\end{array}$ & $<2600 \mathrm{eV}$ & 20 & $-3-270 \mathrm{eV}$ \\
\hline Gd155 & 14.80 & $\begin{array}{c}\text { Chang, } \\
\text { Mughabghab 1999 }\end{array}$ & $<183.3 \mathrm{eV}$ & 92 & $0.02-180 \mathrm{eV}$ \\
\hline Gd156 & 20.47 & Magurno, ENDF5 & $<983.4 \mathrm{eV}$ & 6 & $33-245 \mathrm{eV}$ \\
\hline Gd157 & 15.65 & $\begin{array}{c}\text { Oh, Mughabghab, } \\
\text { 1999 }\end{array}$ & $<306.6 \mathrm{eV}$ & 54 & $0.03-300 \mathrm{eV}$ \\
\hline Gd158 & 24.84 & Magurno, ENDF5 & $<9980.4 \mathrm{eV}$ & 4 & $22-277 \mathrm{eV}$ \\
\hline Gd160 & 21.86 & Magurno, ENDF5 & $<9668.66 \mathrm{eV}$ & 1 & $222 \mathrm{eV}$ \\
\hline
\end{tabular}

Resonance integrals (Table XI) are given for each isotope as well as their contribution to the elemental values. The integrations extend from $0.5 \mathrm{eV}$ to $20 \mathrm{MeV}$. The low energy cutoff is above the thermal region doublet. The elemental resonance integral for $\mathrm{Gd}$ as measured is $2.8 \%(11 \mathrm{~b})$ larger than that of ENDF. The largest fractional increases in isotopic contributions occur in ${ }^{154} \mathrm{Gd}$ and ${ }^{158} \mathrm{Gd} .{ }^{154} \mathrm{Gd}$ and ${ }^{158} \mathrm{Gd}$ have far fewer resonances than ${ }^{155} \mathrm{Gd}$ or ${ }^{157} \mathrm{Gd}$. A $14 \%$ increase in ${ }^{158} \mathrm{Gd}$ resonance integral compared to ENDF was measured. This is dominated by the $22.3 \mathrm{eV}$ resonance whose neutron width changed by approximately the same amount. The resonance integral of ${ }^{154} \mathrm{Gd}$ is $20 \%$ larger than that calculated from ENDF parameters. The ${ }^{154} \mathrm{Gd}$ resonance integral is larger than ENDF due to larger widths for resonances at 22.5, 49.63, 65.21 , and $76.00 \mathrm{eV}$ (see Table VII). ${ }^{155} \mathrm{Gd}$ contributes more than half of the elemental Gd capture resonance integral and its contribution is virtually unchanged when compared to that calculated from ENDF parameters. 
Table IX - Thermal capture cross sections. A comparison of ENDF/B-VI to RPI results. Cross section units are barns. The units of abundance are percent.

\begin{tabular}{|c|c|c|c|c|c|c|c|}
\hline \multicolumn{9}{|c|}{ Thermal Capture Cross Sections } \\
\hline \multirow{2}{*}{ Isotope } & \multirow{2}{*}{ Abund } & \multicolumn{3}{|c|}{ ENDF } & \multicolumn{3}{c|}{ RPI } \\
\cline { 3 - 8 } & & $\begin{array}{l}\text { Thermal } \\
\text { Capture }\end{array}$ & $\begin{array}{l}\text { Contribution } \\
\text { to Elemental }\end{array}$ & Percent & $\begin{array}{l}\text { Thermal } \\
\text { Capture }\end{array}$ & $\begin{array}{l}\text { Contribution } \\
\text { to Elemental }\end{array}$ & Percent \\
\hline Gd152 & 0.200 & 1050. & 2.10 & 0.00430 & 1050. & 2.10 & 0.00430 \\
\hline Gd154 & 2.18 & 85.0 & 1.85 & 0.00379 & 85.8 & 1.87 & 0.00422 \\
\hline Gd155 & 14.80 & 60700. & 8980. & 18.4 & 60200. & 8910. & 20.1 \\
\hline Gd156 & 20.47 & 1.71 & 0.350 & 0.000717 & 1.74 & 0.356 & 0.000804 \\
\hline Gd157 & 15.65 & 254000. & 39800. & 81.6 & 226000. & 35400. & 79.9 \\
\hline Gd158 & 24.84 & 2.01 & 0.499 & 0.00102 & 2.19 & 0.544 & 0.00122 \\
\hline Gd160 & 21.86 & 0.765 & 0.167 & 0.000342 & 0.755 & 0.165 & 0.000372 \\
\hline Gd & -- & & 48800. & 100.0 & & 44300. & 100.0 \\
\hline
\end{tabular}

Table X - Thermal elastic scattering cross sections. A comparison of ENDF/B-VI to RPI results.

Cross section units are barns. The units of abundance are percent.

\begin{tabular}{|c|c|c|c|c|c|c|c|}
\hline \multicolumn{3}{|c|}{ Isotope } & \multirow{2}{*}{ Abund } & \multicolumn{3}{|c|}{ ENDF } & \multicolumn{3}{c|}{ RPI } \\
\cline { 3 - 8 } & & $\begin{array}{c}\text { Thermal } \\
\text { Elastic }\end{array}$ & $\begin{array}{c}\text { Contribution } \\
\text { to Elemental }\end{array}$ & Percent & $\begin{array}{c}\text { Thermal } \\
\text { Elastic }\end{array}$ & $\begin{array}{c}\text { Contribution } \\
\text { to Elemental }\end{array}$ & Percent \\
\hline Gd152 & 0.200 & 23.4 & 0.0468 & 0.0277 & 23.4 & 0.0468 & 0.0342 \\
\hline Gd154 & 2.18 & 7.29 & 0.159 & 0.0941 & 6.69 & 0.146 & 0.107 \\
\hline Gd155 & 14.80 & 60.8 & 8.99 & 5.32 & 59.7 & 8.84 & 6.45 \\
\hline Gd156 & 20.47 & 5.64 & 1.16 & 0.686 & 6.93 & 1.42 & 1.04 \\
\hline Gd157 & 15.65 & 1010. & 157. & 92.9 & 798. & 125. & 91.2 \\
\hline Gd158 & 24.84 & 3.30 & 0.820 & 0.485 & 3.27 & 0.812 & 0.593 \\
\hline Gd160 & 21.86 & 3.63 & 0.795 & 0.470 & 3.63 & 0.794 & 0.580 \\
\hline Gd & -- & & 169. & 100.0 & & 137. & 100.0 \\
\hline
\end{tabular}

Table XI - Infinitely dilute neutron capture resonance integrals. A comparison of ENDF/B-VI to RPI results. Cross section units are barns. The units of abundance are percent.

\begin{tabular}{|c|c|c|c|c|c|c|c|}
\hline \multicolumn{3}{|c|}{ Isotope } & \multirow{2}{*}{ Abund } & \multicolumn{3}{|c|}{ ENDF } & \multicolumn{3}{c|}{ RPI } \\
\cline { 3 - 8 } & & $\begin{array}{c}\text { Capture } \\
\text { RI }\end{array}$ & $\begin{array}{c}\text { Contribution } \\
\text { to Elemental }\end{array}$ & Percent & $\begin{array}{c}\text { Capture } \\
\text { RI }\end{array}$ & $\begin{array}{c}\text { Contribution } \\
\text { to Elemental }\end{array}$ & Percent \\
\hline Gd152 & 0.200 & 476. & 0.952 & 0.243 & 476. & 0.952 & 0.237 \\
\hline Gd154 & 2.18 & 217. & 4.73 & 1.21 & 261. & 5.69 & 1.42 \\
\hline Gd155 & 14.80 & 1540. & 228. & 58.3 & 1570. & 232. & 57.7 \\
\hline Gd156 & 20.47 & 105. & 21.5 & 5.50 & 104. & 21.3 & 5.30 \\
\hline Gd157 & 15.65 & 755. & 118. & 30.2 & 789. & 123. & 30.6 \\
\hline Gd158 & 24.84 & 62.8 & 15.6 & 3.99 & 71.5 & 17.8 & 4.43 \\
\hline Gd160 & 21.86 & 7.89 & 1.72 & 0.440 & 7.66 & 1.68 & 0.418 \\
\hline Gd & -- & & 391. & 100. & & 402. & 100. \\
\hline
\end{tabular}




\section{Discussion- Uncertainties}

In Table VI and Table VII, estimated uncertainties (on the order of $1 \sigma$ ) are given for the present measurements. The scope of the gadolinium measurements, using multiple sample thicknesses, experiment types (transmission or capture), and two energy regimes, is such that the final resonance parameters are derived from an overdetermined system. That is, any subset of the overall data taken would be sufficient to specify the resonance parameters. Several subsets should reproduce the resonance parameters within their uncertainties. Therefore, the variability of the resonance parameters resulting from SAMMY fits to different subsets of the overall data is a method to estimate the uncertainty on the resultant parameters. These variabilities are most likely due to systematic errors.

A systematic error is a bias, rather than a random error, and may be due to features which are common to both capture and transmission measurements. The transmission and capture measurements are independent and complementary methods for determining resonance parameters. Features common to both types of experiments and possible sources of systematic uncertainties include using the same electron accelerator, the same neutron-producing target, the same method for determining flight path length, some of the same Gd samples, and some of the same data acquisition electronics. Other potential sources of error include capture flux normalization and the analytical descriptions of the resolution functions.

Uncertainties generated from SAMMY calculations are Bayesian uncertainties propagated from the counting statistics of the measurements. The purely statistical Bayesian uncertainties generated by SAMMY are given in Appendix B in Table B-2. These errors are generally small and do not reflect differences between data sets. That is, the neutron width from two measurements which differ from each other by $1 \%$, but which each have excellent statistics, would have a very small SAMMY error. But, a more reasonable error of the uncertainty surrounding any central value derived from these two measurements would be $1 \%$. Therefore, the estimated uncertainties quoted in Table VI and Table VII are based upon an envelope of plausible values representing the differences between data sets of equal quality, often different samples from the same measurement. The estimated uncertainties given in Table VI and Table VII represent a realistic estimate of how well we believe the central values. They represent the variability of the fitted results, defined in Section VII.A, below.

The uncertainties on the resonance energies, $E_{0}$, are based on a judgment of the accuracy of flight path lengths and zero-time measurements. They are based on discrepancies between different experiments (capture and transmission, epithermal and thermal) which measure slightly different energies for the same resonances. The maximum precision on resonance energies, $E_{0}$, quoted in Table VII is estimated to be five significant figures. Uncertainties in sample thickness given in Table III are not included in the final uncertainties given in Table VI and Table VII. 


\section{VII.A Discussion- Uncertainties in the Thermal Region}

The uncertainties in sample thickness for liquid samples are larger than those of metals as shown in. The method used to determine the effective thickness of a liquid consists of weighing the quantity of $\mathrm{Gd}_{2} \mathrm{O}_{3}$ necessary for $10 \mathrm{ml}$ of $\mathrm{GdNO}_{3}$ solution in a $10 \mathrm{ml}$ flask. Then a known weight of $\mathrm{DNO}_{3}$ is added to dissolve the $\mathrm{Gd}_{2} \mathrm{O}_{3}$. Next, a known weight of $\mathrm{D}_{2} \mathrm{O}$ is added to bring the volume to $10 \mathrm{ml}$. The concentration of $\mathrm{Gd}_{2} \mathrm{O}_{3}$ is the weight in grams divided by $10 \mathrm{ml}$. The weights are accurate to $0.001 \%$ for a 5 gram sample. The accuracy of this method is limited by the accuracy of the volumetric flask, $0.2 \%$. Each of the samples used is a further dilution of the stock solution, introducing a second $0.2 \%$ error. Subsequent to the gadolinium sample preparation, a second method was developed. It consists of preparing more of each solution than is required, so that part of the batch can be extracted with a precision $(0.02 \%$ of volume) pipette, fired and weighed. Experience has shown that estimates of sample thickness from these two methods can differ by $2 \%$. The second method is preferred since it measures the final product. Application of this second method to the current measurements would require destructively analyzing the liquid samples used in this measurement which was not done. Therefore the uncertainty in sample thickness for the liquid samples is less than or equal to $2 \%$.

Five variations on the thermal fit were performed to define the envelope of plausible resonance parameters. The results of these five methods are given in Appendix B. The final uncertainties, given in Table VI, envelop the range of plausible values for resonance parameters resulting from these five methods.

The first uncertainty determination method was a variation on the combined transmission and capture fit described in Section V. It is called the two-step method. The first step fitted the ${ }^{157} \mathrm{Gd}$ resonance to ${ }^{157} \mathrm{Gd}$-sensitive data with the ${ }^{155} \mathrm{Gd}$ resonance fixed to the ENDF value. The second step fixed the ${ }^{157} \mathrm{Gd}$ resonance parameters to the result from step 1 and varied the ${ }^{155} \mathrm{Gd}$ resonance to ${ }^{155} \mathrm{Gd}$-sensitive data. The detailed results of this approach are given in Appendix B.

The second method quantified uncertainties not associated with the liquid samples and their particular beam collimation system. Metal samples, while black in the thermal region, provide some information in transmission in the wings of the resonance. The ${ }^{155} \mathrm{Gd}$ resonance parameters were fixed to ENDF values during this calculation. The dominant resonance, at $\approx 0.03 \mathrm{eV}$ in ${ }^{157} \mathrm{Gd}$, was varied for this sensitivity. Each metal sample measured in thermal transmission was fitted separately. Also, a covariancematrix-linked serial SAMMY calculation was performed with all three metal thermal transmission samples. The detailed results of these fits are given in Appendix B.

The third method quantified uncertainties not associated with capture flux normalization. This was accomplished by eliminating capture data from the fit. All liquid and metal thermal transmission data was fitted in a covariance-matrix-linked serial SAMMY calculation. The detailed results are given in Appendix B. 
The fourth method isolated and estimated the uncertainty due to capture normalization. A neutron capture flux spectrum was measured. Its magnitude was normalized to the black subthermal region of a metal Gd sample (see section IV.A). A $\approx 10 \%$ discrepancy in neutron width of both resonances existed between these measurements and those of transmission measurements. Both experiments are essentially measuring capture since the contribution from scattering is $<1 \%$. The neutron capture measurement is more complex than the transmission measurement and has more inherent systematic uncertainty. Therefore, capture yield data were renormalized to transmission results for the fit which produced the final resonance parameters. The fourth uncertainty sensitivity study fitted thermal capture (4 samples, 2 enriched in ${ }^{155} \mathrm{Gd}$ and 2 enriched in ${ }^{157} \mathrm{Gd}$ ) only and did not vary from the initial subthermal-capture-data-based normalization. The results for neutron width were predictably $10 \%$ larger than the final KAPL/RPI widths.

The fifth method of uncertainty isolated and estimated the uncertainties associated with reproducing a given transmission result. The ${ }^{155} \mathrm{Gd}$-enriched liquid sample named LX-4 was measured in transmission twice within two weeks under similar experimental conditions. The transmission data from these two experiments are shown in Figure 56. A magnified view of these data in the region of the thermal doublet in $\mathrm{Gd}$ is shown in Figure 57 . The discrepancy of $\approx 2 \%$ between data sets in this region is reflected in the $\approx 3 \%$ error quoted on the ${ }^{155} \mathrm{Gd} 0.025 \mathrm{eV}$ resonance's neutron width (see Table VI).

One of the only differences between the two LX-4 transmission measurements was the pulse width (see Table I). Wider pulses produce more neutrons and therefore higher counting rates. Differences between the results of the two LX-4 thermal transmission experiments may be count-rate related or they may represent our ability to reproduce a thermal transmission result. This issue will be explored further in Section VIII, Conclusions.

Uncertainties in the isotopic composition of liquid samples given in Table II are not included in the final uncertainties given in Table VI.

\section{VII.B Discussion- Uncertainties in the Epithermal Region}

As in the thermal range, Bayesian uncertainties are generally insignificant compared to those discussed in this section. Three additional methods to estimate resonance parameter uncertainties were employed in the epithermal region. The epithermal resonance parameters of $\mathrm{Gd}$ and the estimated uncertainties are given in Table VII. Details of the components of the uncertainties are given in Appendix B.

The first method of determining uncertainty estimates the variability between data sets. Transmission and capture fitted separately should agree within their errors to the combined transmission and capture fit. Therefore, the deviation of a transmission-only result or a capture-only result from the central value was accepted as an estimate of a 
parameter's uncertainty. The central values obtained in the combined transmission and capture fit are weighted in favor of the transmission data since they had better counting statistics. Therefore, transmission-only deviations are small compared to capture-only deviations and are not presented here. The capture-only deviations from the final parameters are given in Appendix B.

Secondly, a difficulty was encountered quantifying radiation widths and their uncertainties. Capture resonances, where $\Gamma_{\gamma} \gg \Gamma_{n}$, are insensitive to $\Gamma_{\gamma}$ in both transmission and capture. Therefore, the SAMMY Bayesian fits are very dependent on initial parameters and initial uncertainties. The default value of input uncertainties for resonance parameters is $10 \%$ and was used in the current analysis. SAMMY fits of radiation widths often result in large values $(>160 \mathrm{meV}$ ) that are nonphysical and which do not improve the fits to the data significantly. Often results for radiation width are arbitrary and simply increase by some percentage $(\approx 20 \%)$ above any reasonable starting point. The second method of estimating uncertainties assigned an uncertainty in radiation width large enough to include the value calculated in an unconstrained SAMMY fit. Also, the degree to which neutron width is affected by the constraint of radiation width has been factored into the neutron width uncertainties quoted in Table VII. This was particularly common in the region above $184 \mathrm{eV}$ where the ENDF-defined resolved resonance region for ${ }^{155} \mathrm{Gd}$ ends and there are overlapping resonances. But this phenomenon was observed as low as $96 \mathrm{eV}$. A further note on runaway radiation widths: experience suggests that radiation width fits to singlet resonances which result in unduly large values may indicate the presence of a multiplet.

The third method was used only in the $10-80 \mathrm{eV}$ region. There were questions about the background treatment of epithermal transmission data that precluded their use below $10 \mathrm{eV}$. Background issues persisted up to about $80 \mathrm{eV}$, see Figure 19. Therefore, uncertainties in this region are set large enough to include the resonance parameters resulting from a SAMMY fit including background variables $(A+B / \sqrt{ } E)$.

In the cases where the above methods yield errors smaller than those propagated from the data by SAMMY, the SAMMY Bayesian error is quoted in Table VII.

The largest of the independently calculated uncertainties described above is quoted as the overall uncertainty for each resonance parameter in Table VII. Many of these errors are rather large, 50-100\%, or more. Most often these are parameters from minor resonances which are not resolvable from another larger resonance or a resonance from a more naturally abundant isotope. The parameters from the ${ }^{154} \mathrm{Gd}$ resonance at $22.5 \mathrm{eV}$ are a good example of large errors in a resonance from a low abundance isotope. This resonance could not be resolved from the $22.3 \mathrm{eV}$ in the more abundant ${ }^{158} \mathrm{Gd}$. The $22.5 \mathrm{eV}$ resonance is a major contributor to the resonance integral of ${ }^{154} \mathrm{Gd}$.

Large uncertainties in resonance parameters also occur when the component resonances of an unresolved doublet are of comparable size. This occurs at $44 \mathrm{eV}$. Here, the distribution of area under the peak between the two resonances is unknown in a measurement of natural metal Gd samples. Therefore, Table VII shows large 
uncertainties on the parameters for each resonance. The total area under the measured curve is well known, but none of the component widths is known with certainty. These are conservative estimates of the uncertainties on each resonance parameter based upon the methods described above. However, the uncertainty in the contribution to resonance integral in this region is much better known than a propagation of individual resonance parameter uncertainties would imply.

\section{Conclusions}

Resonance parameters were extracted from combined capture and transmission data sets using the multi-level R-matrix Bayesian code SAMMY. The analysis included Doppler broadening, resolution broadening and multiple scattering correcting of capture data. Separate resolution functions for transmission and capture were used.

The present measurements assumed the same spin assignments as ENDF for all resonances analyzed. Neutron widths and thermal $(2200 \mathrm{~m} / \mathrm{s})$ capture cross sections of the thermal doublet are smaller than currently published (ENDF) values. The conclusions of these measurements are as follows:

- The neutron width of the $0.032 \mathrm{eV}$ resonance in ${ }^{157} \mathrm{Gd}$ is $9 \%$ smaller than that of ENDF.

The neutron width of the $0.025 \mathrm{eV}$ resonance in ${ }^{155} \mathrm{Gd}$ is $7 \%$ smaller than that of ENDF.

The radiation width of the $0.032 \mathrm{eV}$ resonance in ${ }^{157} \mathrm{Gd}$ is not significantly different from that of ENDF.

- The radiation width of the $0.025 \mathrm{eV}$ resonance in ${ }^{155} \mathrm{Gd}$ is $4 \%$ smaller than that of ENDF.

The thermal $(2200 \mathrm{~m} / \mathrm{s})$ cross section of ${ }^{157} \mathrm{Gd}$ is $11 \%$ smaller than that of ENDF.

- The thermal $(2200 \mathrm{~m} / \mathrm{s})$ cross section of ${ }^{155} \mathrm{Gd}$ is not significantly different from that of ENDF.

In the epithermal region, a great deal of improvement has been made to the $\mathrm{Gd}$ resonance parameter database. In the energy region near $96 \mathrm{eV}$, and particularly above $165 \mathrm{eV}$, significant changes are suggested to ENDF parameters. New resonances have been suggested where comparisons of data to calculations clearly show they are needed

Any future gadolinium measurements must be improved beyond the current methods. As described herein, there were internal inconsistencies between thermal transmission and capture results and within thermal transmission measurements. The magnitude of these inconsistencies was quantified by the uncertainties on the resonance parameters quoted in Table VI. The internal inconsistencies within the data sets can also be seen by the inability of a single set of resonance parameters to simultaneously fit various data sets shown in Figure 10-Figure 16. 
Uncertainty due to experimental methods was introduced due to the use of liquid samples, whose diameters require special collimation of the neutron beam. Also, these samples require an appropriate capture flux normalizer; i.e., a black liquid sample in a cell with the same geometry as the normalized samples. This was not available. Capture data were normalized to a larger diameter metal Gd sample and a geometric correction factor was measured and applied. Furthermore, this metal flux normalizer was not sufficiently black to allow flux normalization at thermal energies. The liquid sample thermal capture flux was normalized at subthermal energies.

The fifth method of uncertainty analysis in Section VII.A discusses discrepancies between similar transmission measurements made on the same liquid sample, LX-4. In this comparison, the uncertainty in sample thickness is eliminated as a variable. One of the few differences in experimental parameters between the two liquid sample thermal transmission experiments is pulse width. Higher count rates can be expected to be associated with use of wider pulses. There may be the introduction of deadtime concerns associated with wide $(>1 \mu \mathrm{s})$ pulses and high count rates. One useful experiment would quantify the response of the thermal transmission detector vs event rate. By observing a pulse height spectrum from the $15-\mathrm{m}{ }^{6} \mathrm{Li}$ glass transmission detector and its associated electronics, one could see if legitimate pulses are being "clipped" and discriminated out under high count rate conditions. Until this effect is characterized, RPI linac cross section measurements are being limited to use a maximum pulse width of $1 \mu$ s.

The present thermal neutron capture experiments exhibited collimation issues and questions of beam leakage past the samples. For the liquid sample thermal capture experiment, an annular ${ }^{10} \mathrm{~B}$ collimator internal to the capture detector was used. Although a trimming collimator was also used, a very large high energy (short time-offlight) count rate was seen by the detector due to boron (n, $\alpha \gamma)$ gamma rays from the internal collimator. This shower of gammas paralyzed the detector for a few $\mathrm{ms}$, but the detector was fully recovered many ms later when thermal neutrons arrived at the detector. The collimation system for neutron capture experiments at the RPI linac has recently been improved. A brass collimator has been installed and tested. The system also includes an annular ${ }^{10} \mathrm{~B}$ collimator inserted into the detector shield but outside of the detector itself.

Any future gadolinium measurement could avoid collimation questions by using standard 5.04-cm (1.985-in) diameter samples which require no special neutron beam collimation. The original purpose of designing small diameter samples was to preserve a very small quantity of isotopically-enriched material. However, the present measurements have shown that very dilute solutions would be effective in thermal capture. Some of the very dilute solutions were of value in thermal transmission. Therefore, it is believed that enough isotopically-enriched material could be recovered from the current samples to make an adequate set of thin, dilute samples in 5.04-cm (1.985-in) diameter quartz cells.

If thicker, separated isotopic samples could be obtained, much progress could be made on the ambiguities in the epithermal region. 
There are also questions about epithermal transmission results below $80 \mathrm{eV}$ introducing uncertainty into the resulting resonance parameters (See Section VII.B). This uncertainty in epithermal transmission background is most pronounced below $10 \mathrm{eV}$, precluding the use of this region of the data. The treatment of background is the subject of ongoing study.

Results in the epithermal region could be improved with the use of separated isotopes. Samples would need to be thicker for this region than the sample produced for use in the present measurement in the thermal region. That is, grams of separated isotopes would be needed for the measurements. 
1. R. E. SLOVACEK, R. C. BLOCK, Y. DANON, C. WERNER, G.-U. YOUK, J. A. BURKE, N. J. DRINDAK, F. FEINER, J. A. HELM, K. W. SEEMANN, "Neutron Cross-Section Measurements at the Rensselaer LINAC," Proc. Topl. Mtg. Advances in Reactor Physics, April 11-15, 1994, Knoxville, Terınessee

2. R. C. BLOCK, P. J. MARANO, N. J. DRINDAK, F. FEINER, K. W. SEEMANN, and R. E. SLOVACEK, "A Multiplicity Detector for Accurate Low-Energy Neutron Capture Measurements," Proc. Int. Conf. Nuclear Data for Science and Technology, May 30June 3,1988, Mito, Japan, p. 383.

3. R. C. BLOCK, Y. DANON, C. J. WERNER, G. YOUK, J. A. BURKE, N. J. DRINDAK, F. FEINER, J. A. HELM, J. C. SAYRES, and K. W. SEEMANN, "Neutron Time-of-Flight Measurements at the Rensselaer LINAC," Proc. Int. Conf. Nuclear Data for Science and Technology, May 9-13, 1994, Gatlinburg, Tennessee, Vol. 1, p. 81, American Nuclear Society (1994).

4. M. E. OVERBERG, B. E. MORETTI, R. E. SLOVACEK, R. C. BLOCK, "Photoneutron Target Development for the RPI Linear Accelerator," Nucl. Instrum. \& Meth. Physics Research A, 438, 253 (1999).

5. Y. DANON, R. E. SLOVACEK, and R. C. BLOCK, "The Enhanced Thermal Neutron Target at the RPI LINAC," Trans. Am. Nucl. Soc., 68, 473 (1993).

6. Y. DANON, R. E. SLOVACEK, and R. C. BLOCK, "Design and Construction of a Thermal Neutron Target for the RPI LINAC," Nucl. Instrum. \& Methods Physics Research A,352, 596 (1995).

7. N. M. LARSON, "Updated Users' Guide for SAMMY: Multilevel R-Matrix Fits to Neutron Data Using Bayes' Equations," ORNL/TM-9179/R5, Lockheed Martin Energy Research Corp., Oak Ridge National Laboratory, (2000).

8. P. F. ROSE and C. L. DUNFORD, "ENDF-102 Data Formats and Procedures for the Evaluated Nuclear Data File ENDF-6," BNL-NCS-44945, Rev. 2, Brookhaven National Laboratory (1997).

9. H. BJERRUM MOLLER, F. J. SHORE, and V. L. SAILOR, "Low-Energy Neutron Resonances in Erbium and Gadolinium," Nucl. Sci. Eng., 8, 183-192 (1960).

10. S.F. MUGHABGHAB and R. E. CHRIEN, "S-Wave Neutron Strength Functions of the Gd Isotopes," Phys. Rev. 180, 1131-1138 (1969).

11. F. B. SIMPSON, "Neutron Resonance Parameters for $\mathrm{Sm}^{147}, \mathrm{Sm}^{149}, \mathrm{Gd}^{155}$, and $\mathrm{Gd}^{157}$," Bull. Am. Phys. Soc., 2, 42(NA7) (1957). 
12. M. P. FRICKE, W. M. LOPEZ, S. J. FRIESENHAHN, A. D. CARLSON, D.

COSTELLO, "Neutron Resonance Parameters and Radiative Capture Cross Section of

Gd from $3 \mathrm{eV}$ to $750 \mathrm{keV}$," Nucl. Phys. A, 146, 337-358 (19.70).

13. F. RAHN, H. S. CAMARDA, G. HACKEN, W. W. HAVENS, JR., H. I. LIOU, and J. RAINWATER, "Neutron resonance spectroscopy: ${ }^{154,158,160}$ Gd," Phys. Rev. C (Nucl. Phys.), 10, 1904 (1974).

14. V. A. ANUFRIEV, S. I. BABICH, S. M. MASYONOV, (C, 87KIEV,2, 225, 8709) NNDC CINDA EXFOR ENTRY \# 40984 (1987).

15. R. L. MACKLIN, "Neutron Capture Resonances of ${ }^{152} \mathrm{Gd}$ and ${ }^{154} \mathrm{Gd}$," Nucl. Sci Eng., 95, 304-310 (1987)

16. F.N.BELYAEV,V.P.BOLOTSKIY,B.V.EFIMOV,G.N.MURADYAN, Jour. Jaderaja Fizika (YF,52,(3),625,9009) Engl transl. = SNP, Soviet Jour. of Nucl. Phys.

17. E. N. KARZHAVINA, NGUEN NGUEN PHONG, A.B. POPOV, Jour. JadernoFizicheskie-Issledovanija (progress reports), (R, YFI-6,135,6811) (1968).

18. E.N.KARZHAVINA, KIM-SEK-SU, A. B. POPOV, Joint Inst. For Nucl Res., Dubna. Reports, (R,JINR-P3-6948,73) (1973).

19. M. ASGHAR, P. ASGHAR, E. SILVER, J. TROCHON, Nucl. Phys. A, 145, 549 (1970).

20. E. M. BAUM, H. D. KNOX, and T. R. MILLER, "Chart of the Nuclides," $16^{\text {th }}$ Edition, KAPL Inc. (2002).

21. D.K. OLSEN, G. DE SAUSSURE, R.B. PEREZ, F.C. DIFILIPPO, R.W. INGLE, and H. WEAVER, "Measurement and Resonance Analysis of Neutron Transmission Through Uranium-238," Nucl. Sci. Eng., 69, 202 (1979).

22. Y. DANON and R. C. BLOCK, Nucl. Instrum. \& Methods Physics Research A, 485. 585 (2002).

23. D. B. SYME, "The Black and White-Filter Method for Background Determination in Neutron Time-of-Flight Spectrometry," Nucl. Instrum. and Methods, 198, 357 (1982).

24. Y. DANON, "Design and Construction of the RPI Enhanced Thermal Neutron Target and Thermal Cross-Section Measurements of Rare Earth Isotopes," PhD Thesis, Rensselaer Polytechnic Institute (1993).

25. D. P. BARRY, "Neodymium Neutron Transmission and Capture Measurements and Development of a New Transmission Detector," PhD Thesis, Rensselaer Polytechnic Institute (2003). 
26. J. F. BREISMEISTER, Ed., "MCNP - A General Monte Carlo N-Particle Transport Code, Version 5," Los Alamos National Laboratory Rep. LA-UR-03-1987, April 24, 2003.

27. V. McLANE, P. F. ROSE, and C. L. DUNFORD, Neutron Cross Sections, Vol. 2, Academic Press, New York (1988).

28. R. E. MacFARLANE and D. W. MUIR, "The NJOY Nuclear Data Processing System Version 91," LA-12740-M, Los Alamos National Laboratory (Oct. 1994).

29. S. F. MUGHABGHAB, Neutron Cross Sections, Vol , Part B, Academic Press, Orlando, Florida (1984).

30. C. COCEVA and M. STEFANON, Nucl. Phys. A, 315, 1 (1979). 

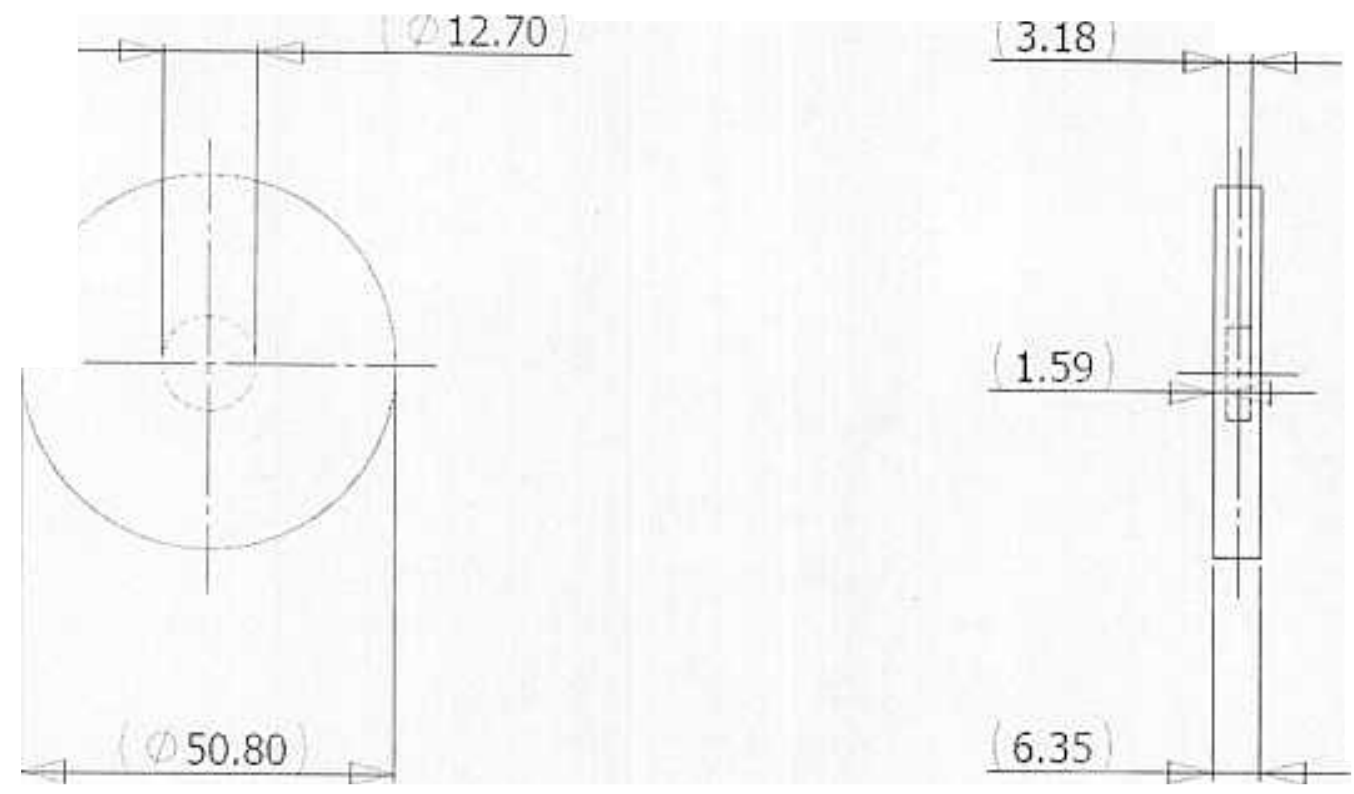

Figure 1- Geometry of the quartz cells used for liquid samples for thermal measurements. Units are mm. 


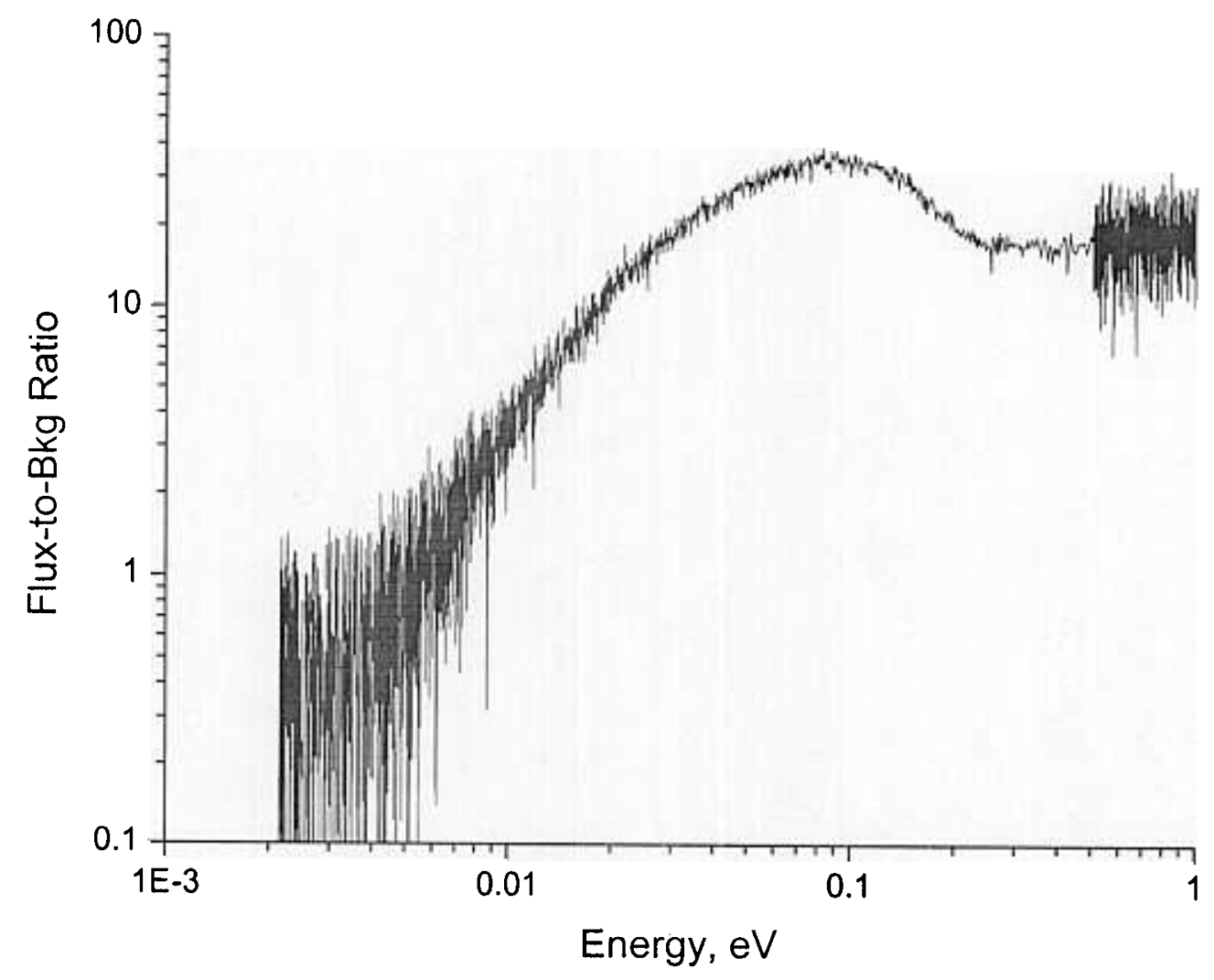

Figure 2- Flux-to-background ratios from the thermal capture experiment. 


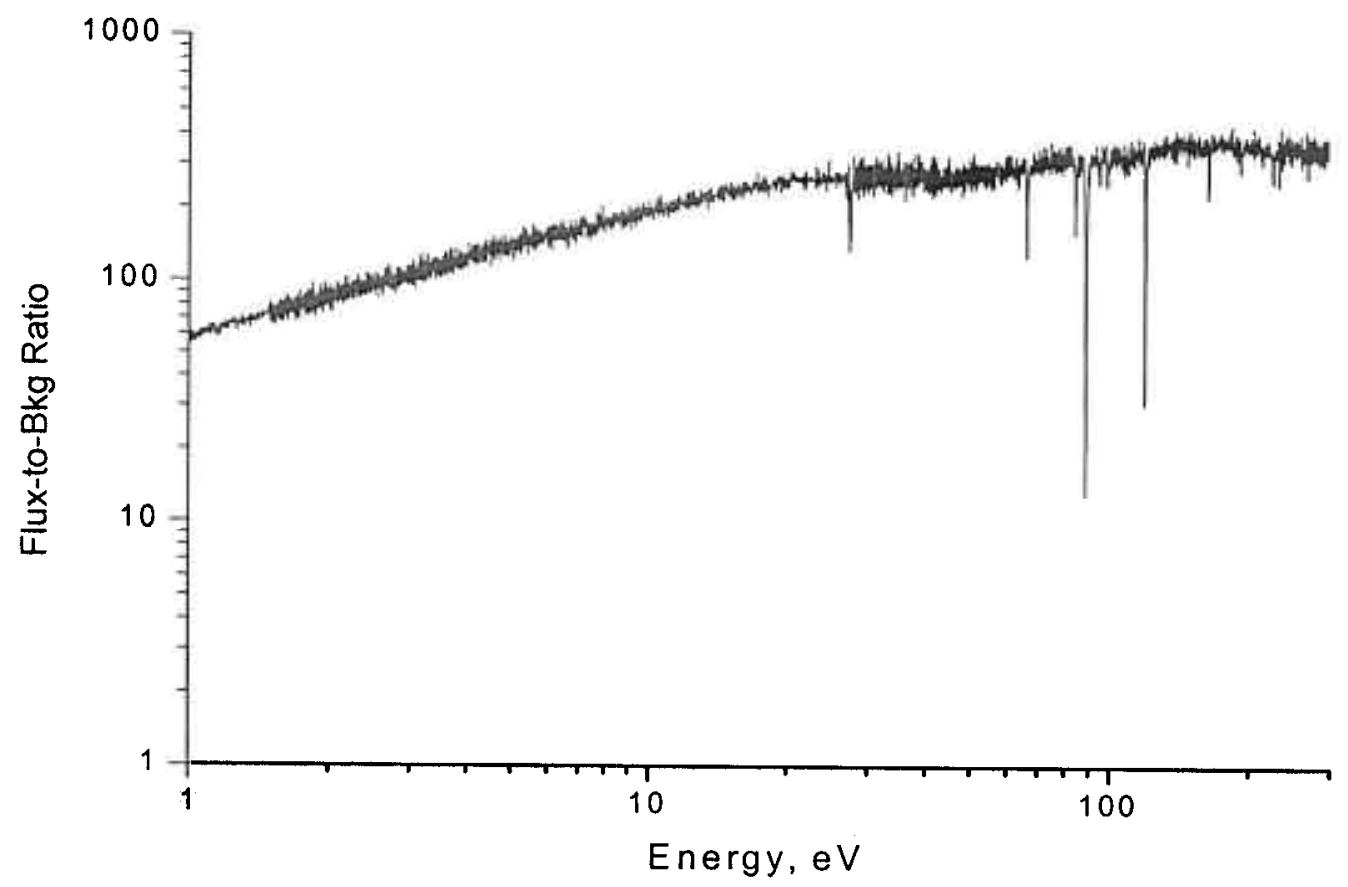

Figure 3- Flux-to-background ratios from the epithermal capture experiment with a Cd filter.

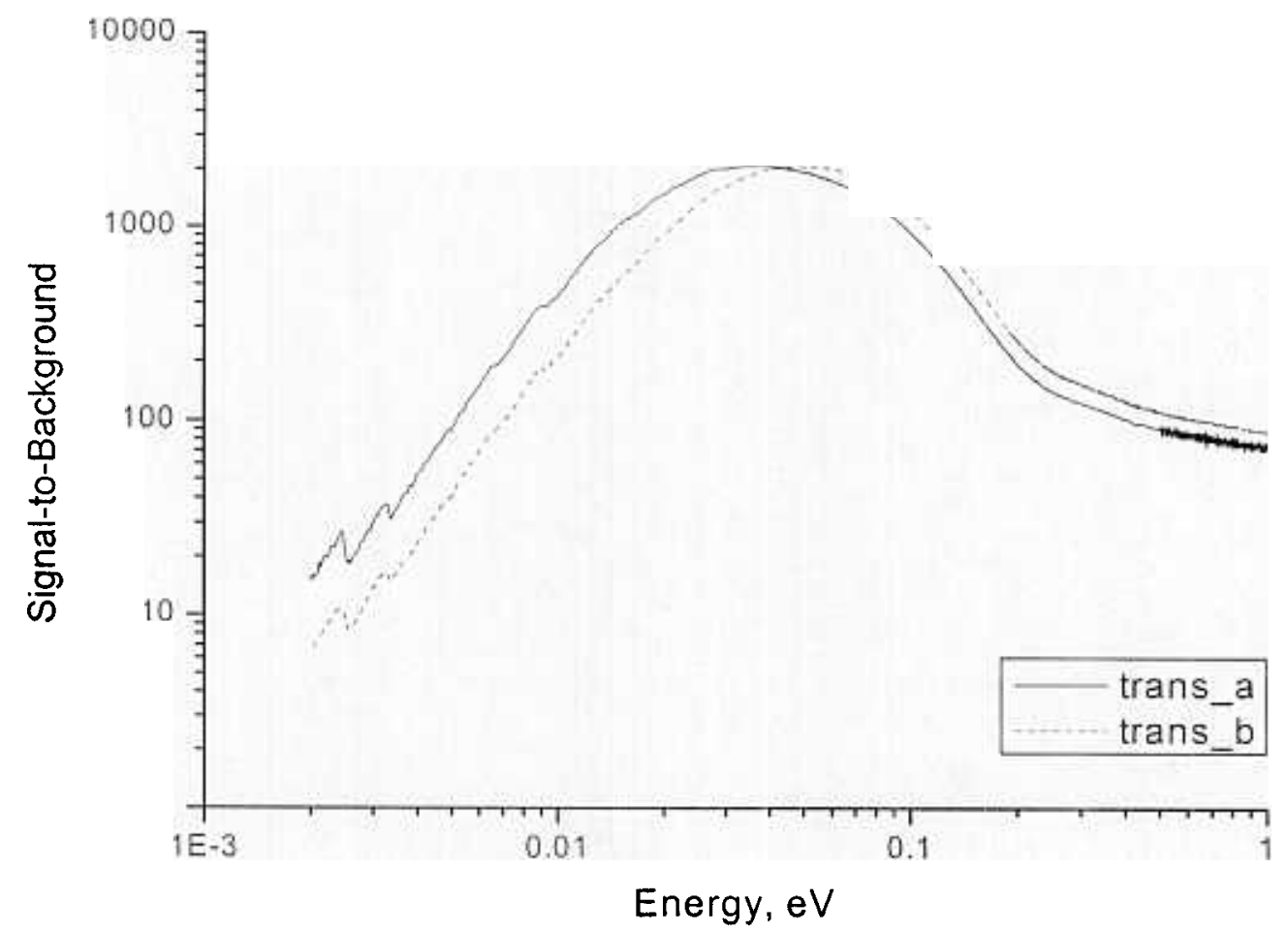

Figure 4- Signal-to-background ratios from the two liquid sample thermal transmission experiments. 


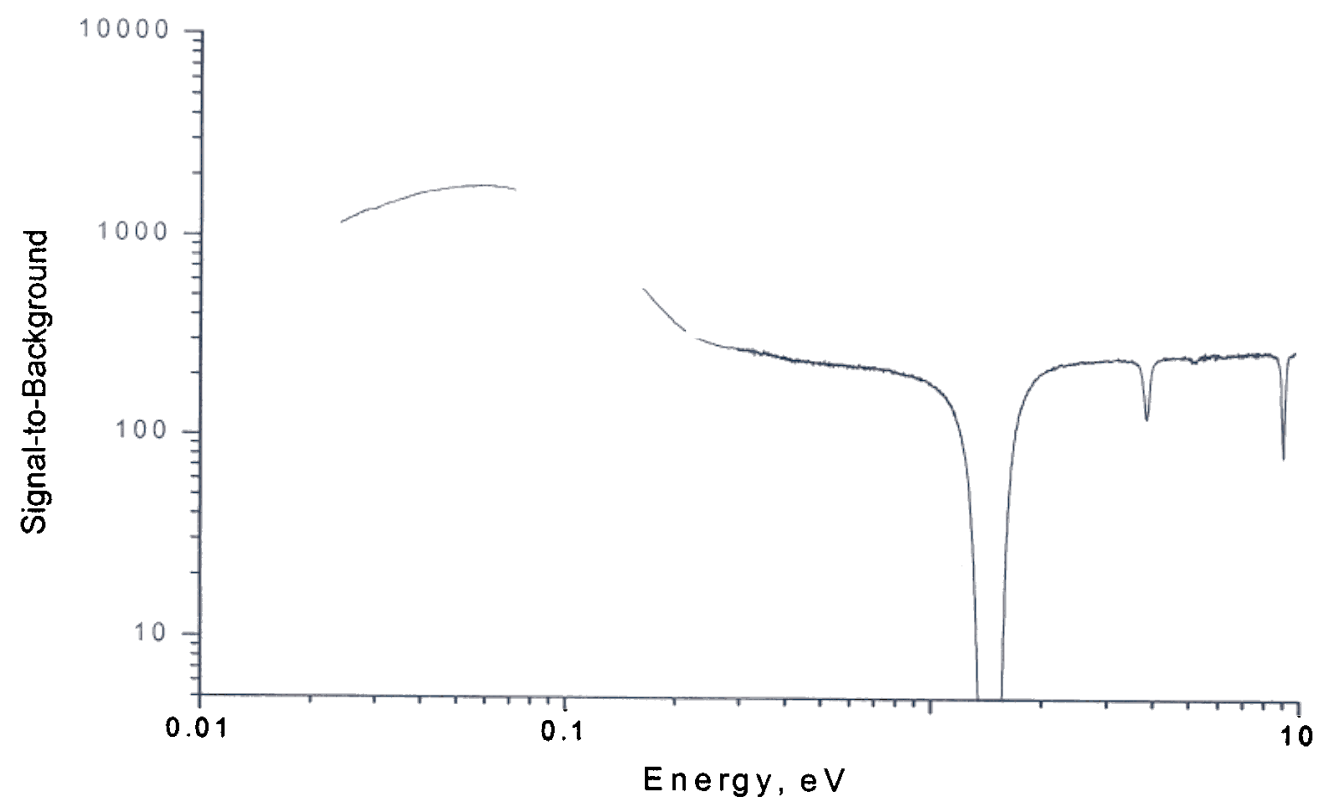

Figure 5- Signal-to-background ratios from the metal sample thermal transmission experiment.

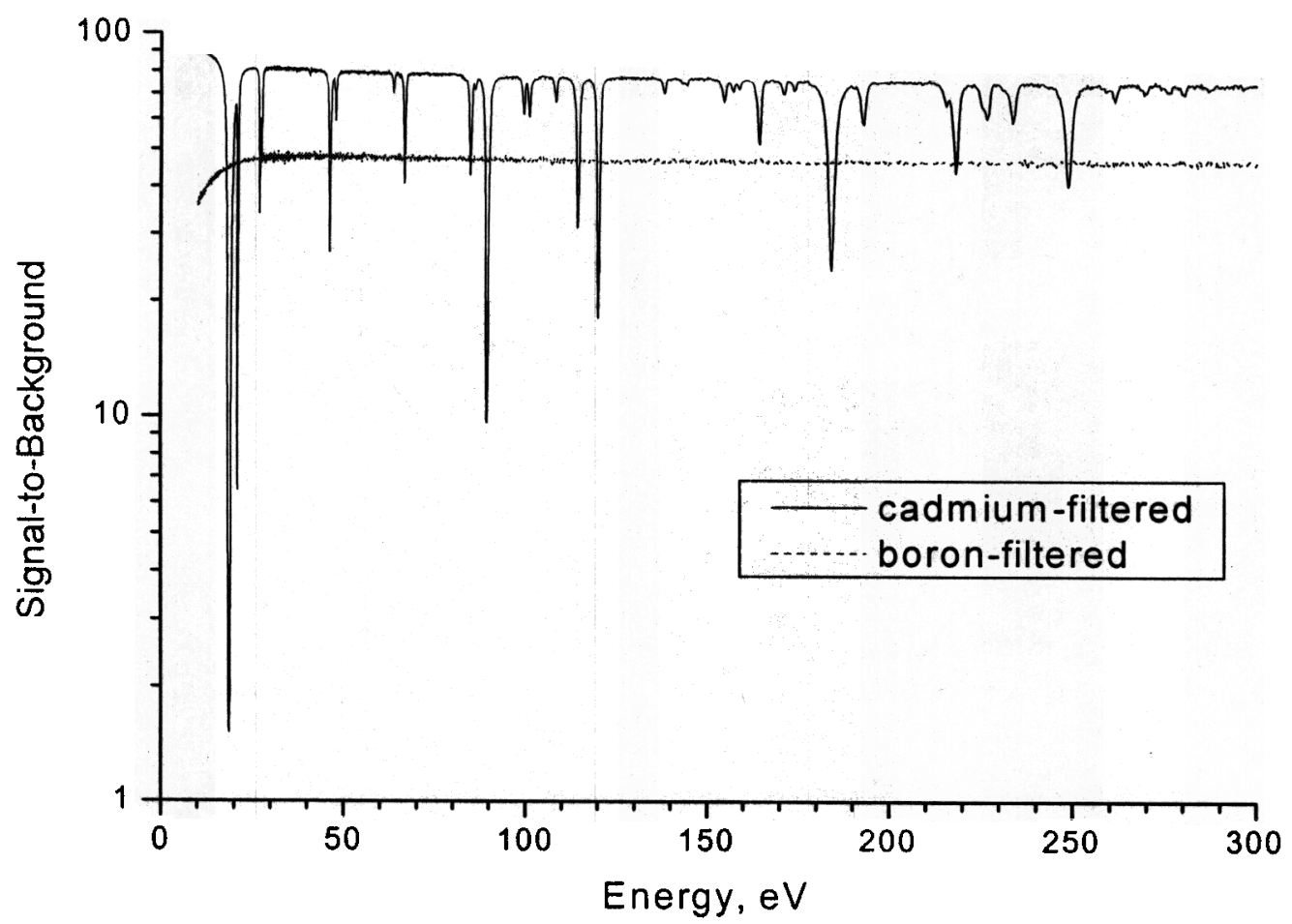

Figure 6- Signal-to-background ratios from the two metal sample epithermal transmission experiments. 


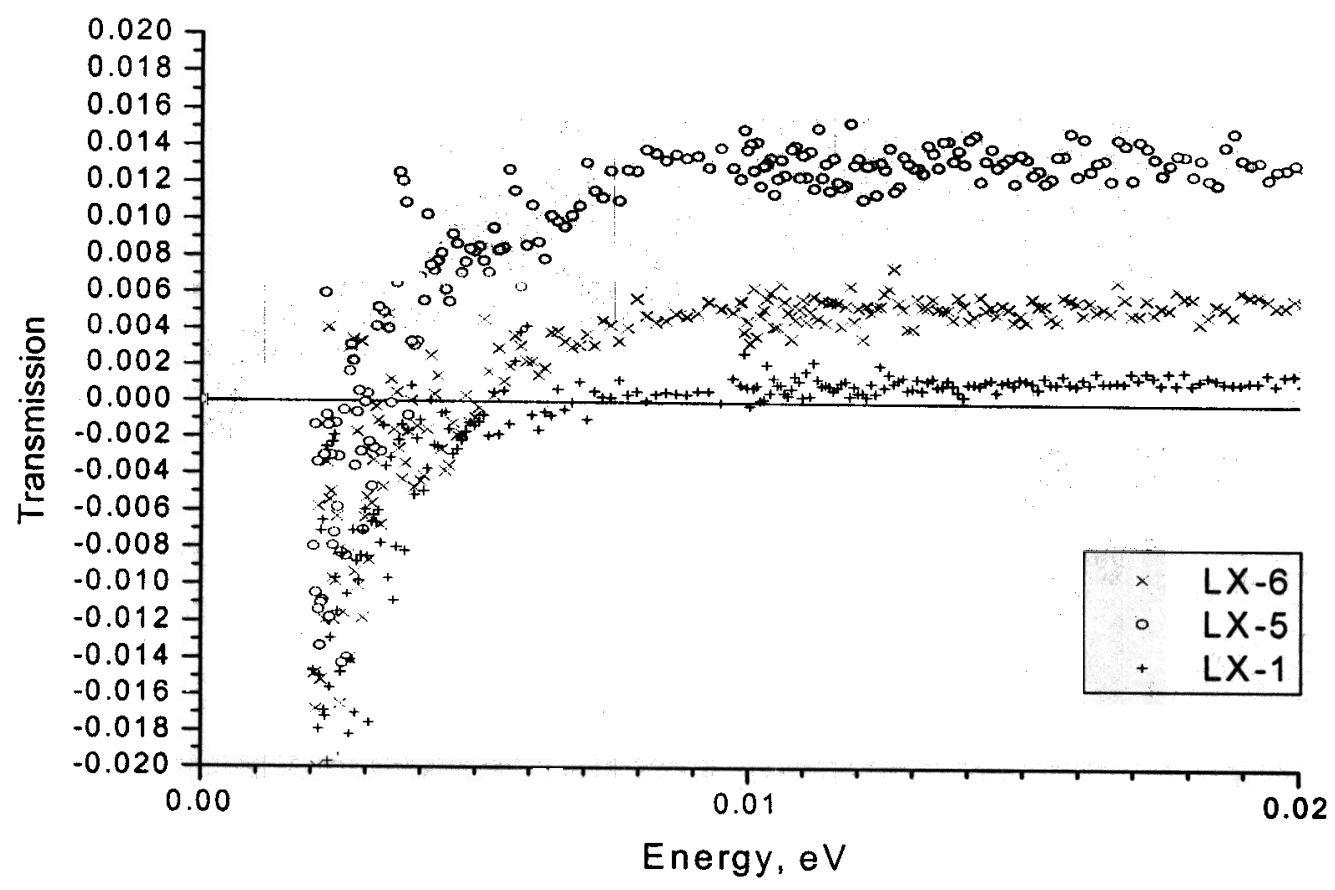

Figure 7- Liquid sample subthermal transmission data from black samples. Transmission is constant indicating saturation, but transmission is not equal to zero indicating a background problem. LX-6 and LX-5 are enriched in ${ }^{157}$ Gd, LX-1 is enriched in ${ }^{155} \mathrm{Gd}$.

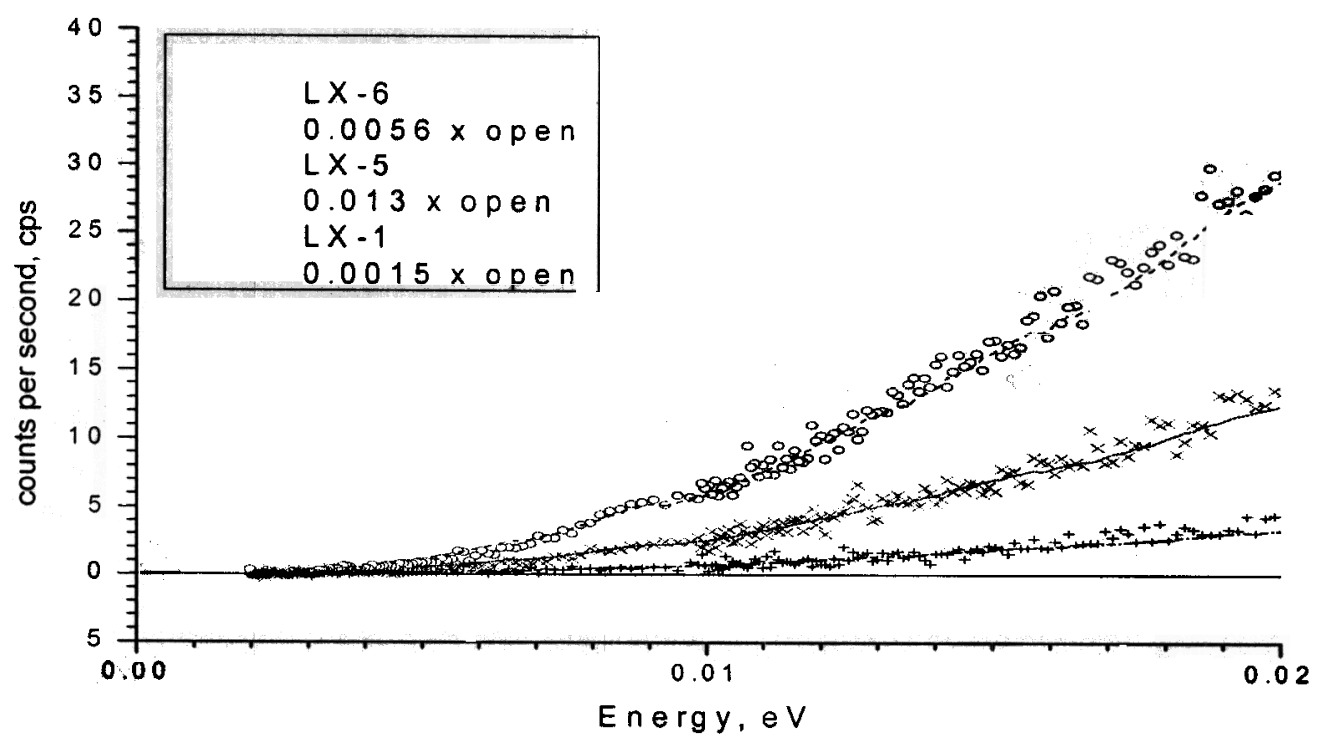

Figure 8- Subthermal count rate data from liquid samples. Transmission from these samples is black, but does not go to zero (see previous figure) indicating a background problem. The shape of the data coincides with the shape of the open beam data suggesting a portion of the open beam leaked by these samples due to physical misalignment. LX-6 and LX-5 are enriched in ${ }^{157}$ Gd, LX-1 is enriched in ${ }^{155} \mathrm{Gd}$. 


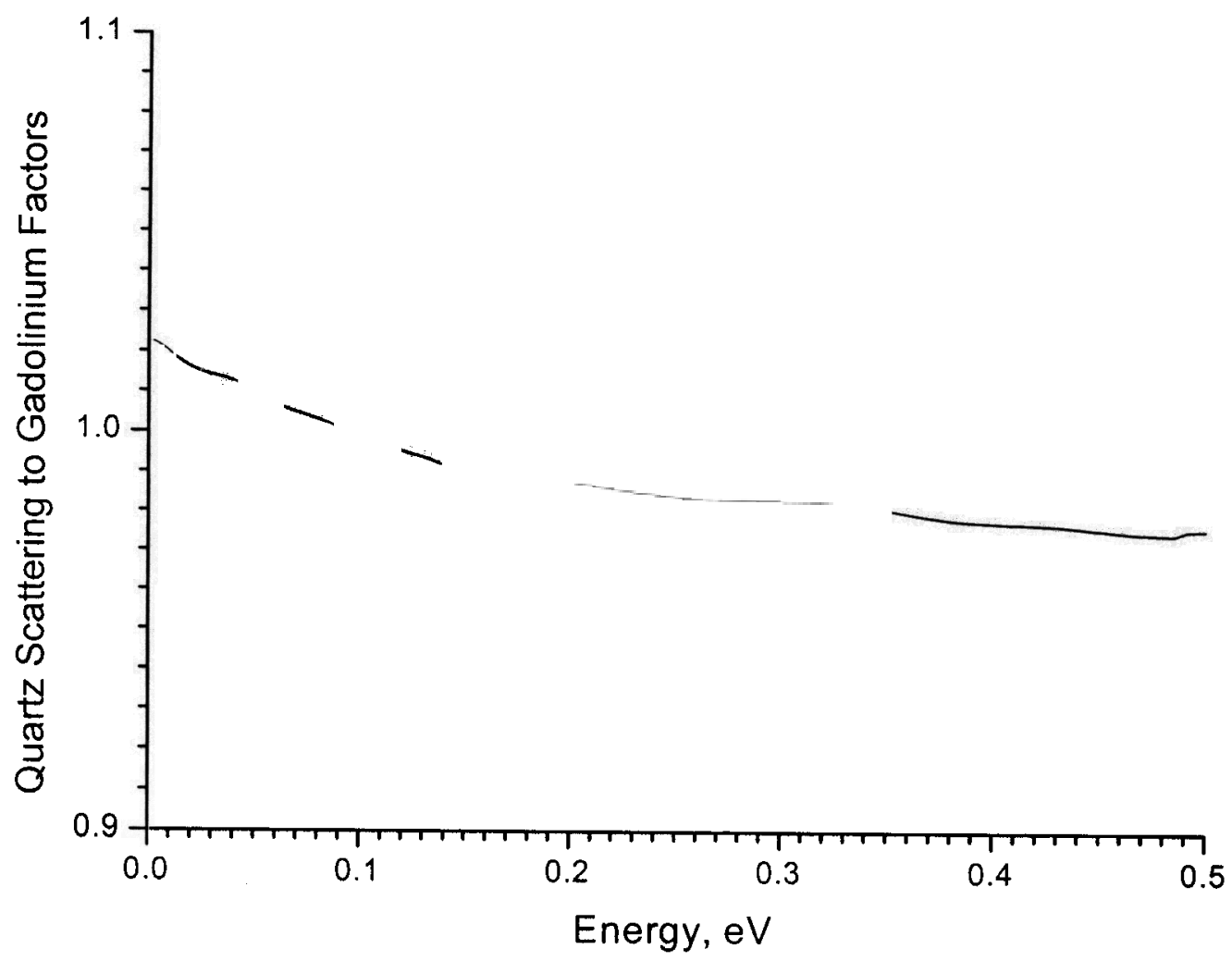

Figure 9- Quartz scattering to gadolinium factors for sample LX-4. Ratio of the capture yield calculated with the SAMMY geometry divided by the yield calculated with the geometry used during the data collection. The factors correct for the limitations of geometric representation in SAMMY. A description is given in Section V, Analysis Methods. More detail is provided in Appendix A. 


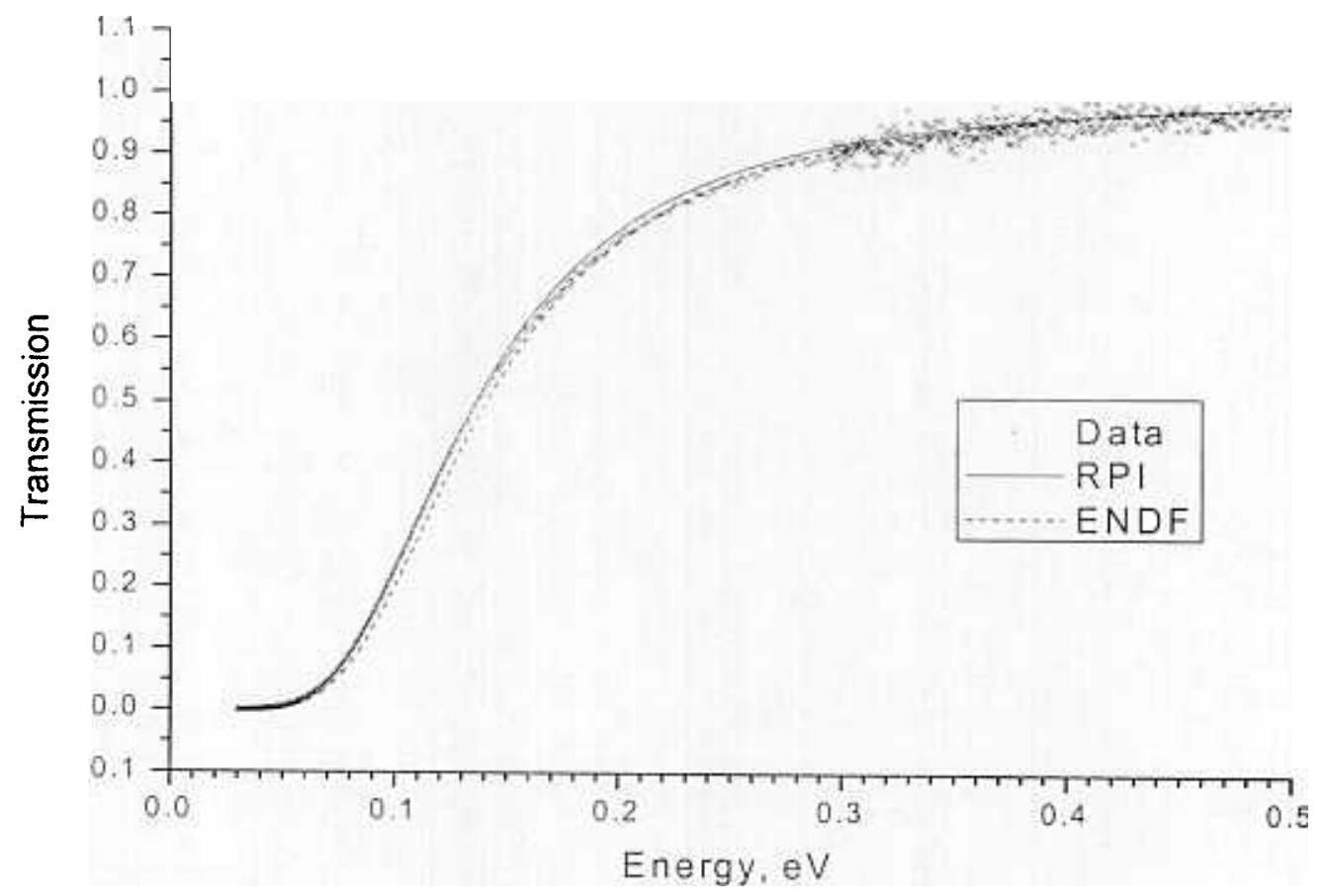

Figure 10 - 0.0051-mm (2-mil) Gd metal thermal transmission data. The RPI fit represents a combined 13-sample SAMMY fit to liquids and metals in transmission and capture.

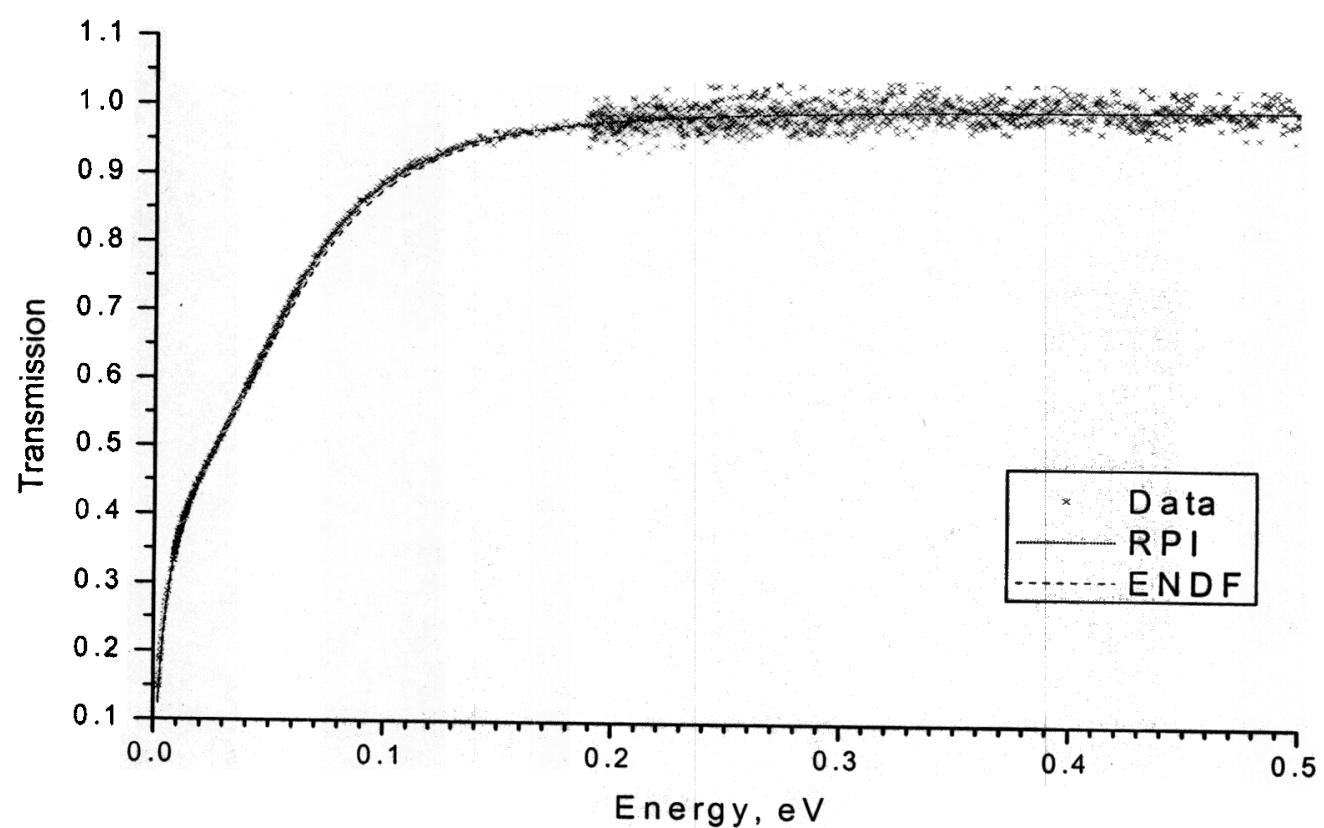

Figure 11 - LX-4 liquid Gd thermal transmission (experiment_b) data. The RPI fit represents a combined 13-sample SAMMY fit to liquids and metals in transmission and capture. The sample is enriched in ${ }^{155} \mathrm{Gd}$ (see Table II andTable III). 


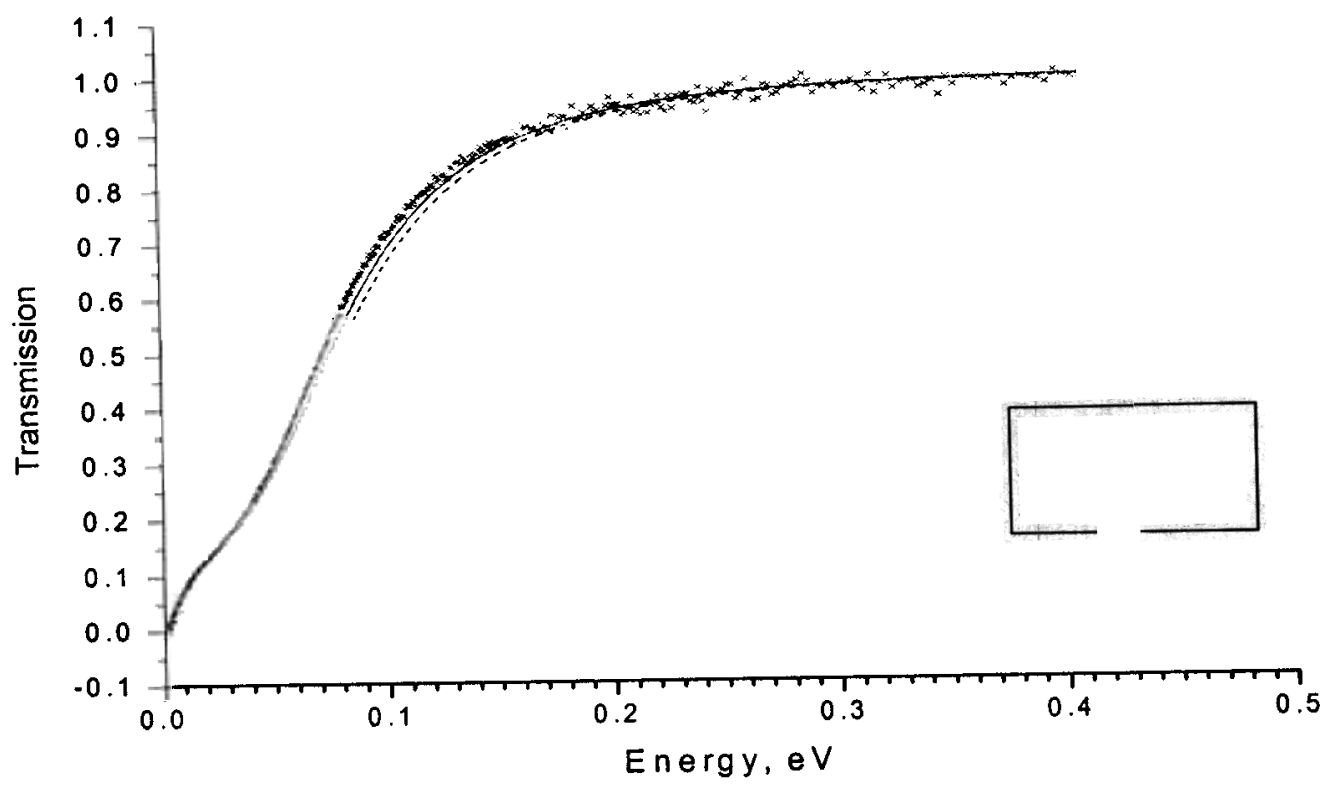

Figure 12-LX-7 liquid Gd thermal transmission (experiment_a) data. The RPI fit represents a combined 13-sample SAMMY fit to liquids and metals in transmission and capture. The sample is enriched in ${ }^{157} \mathrm{Gd}$ (see Table II and Table III).

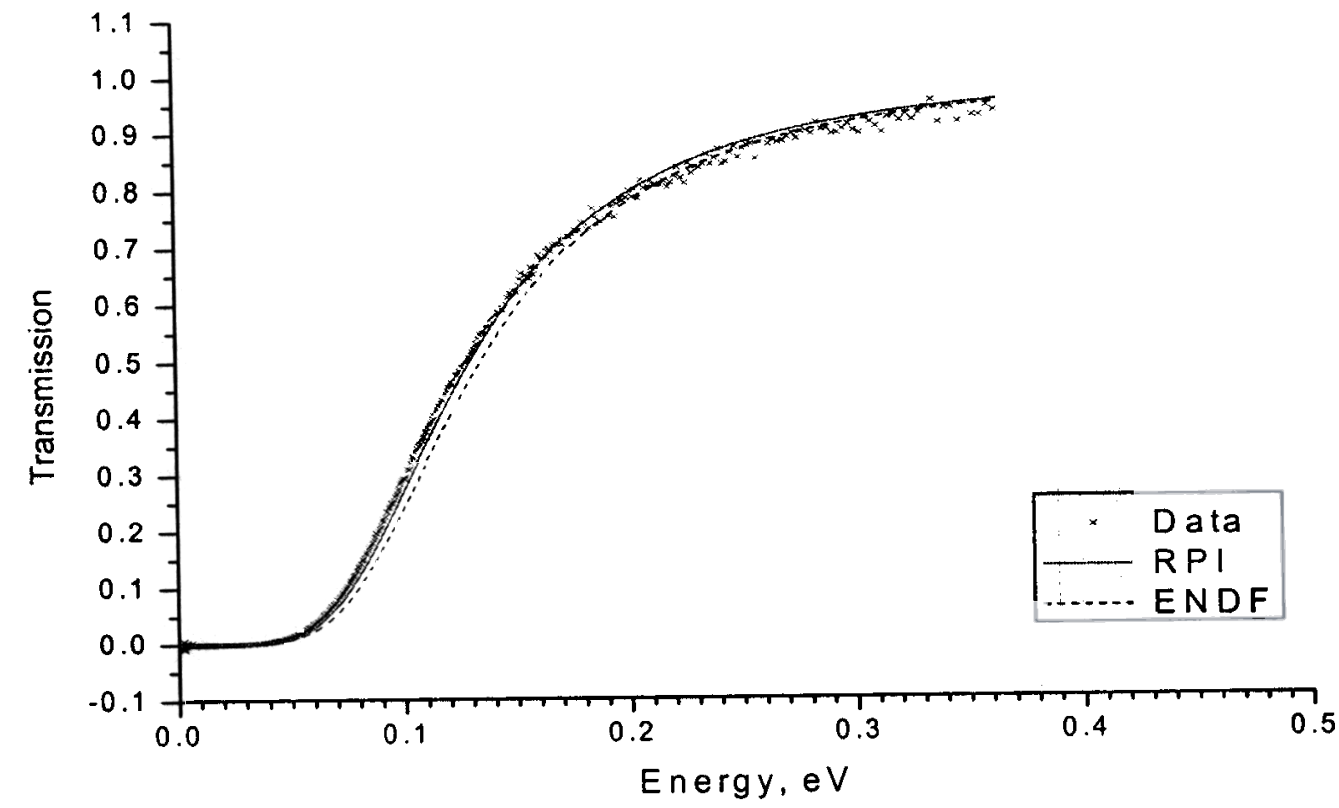

Figure 13- LX-1 liquid Gd thermal transmission (experiment_a) data. The data shown have been corrected for $0.15 \%$ beam leakage past the sample. The RPI fit represents a combined 13 -sample SAMMY fit to liquids and metals in transmission and capture. The sample is enriched in ${ }^{155} \mathrm{Gd}(\mathrm{see}$ Table II and Table III). 


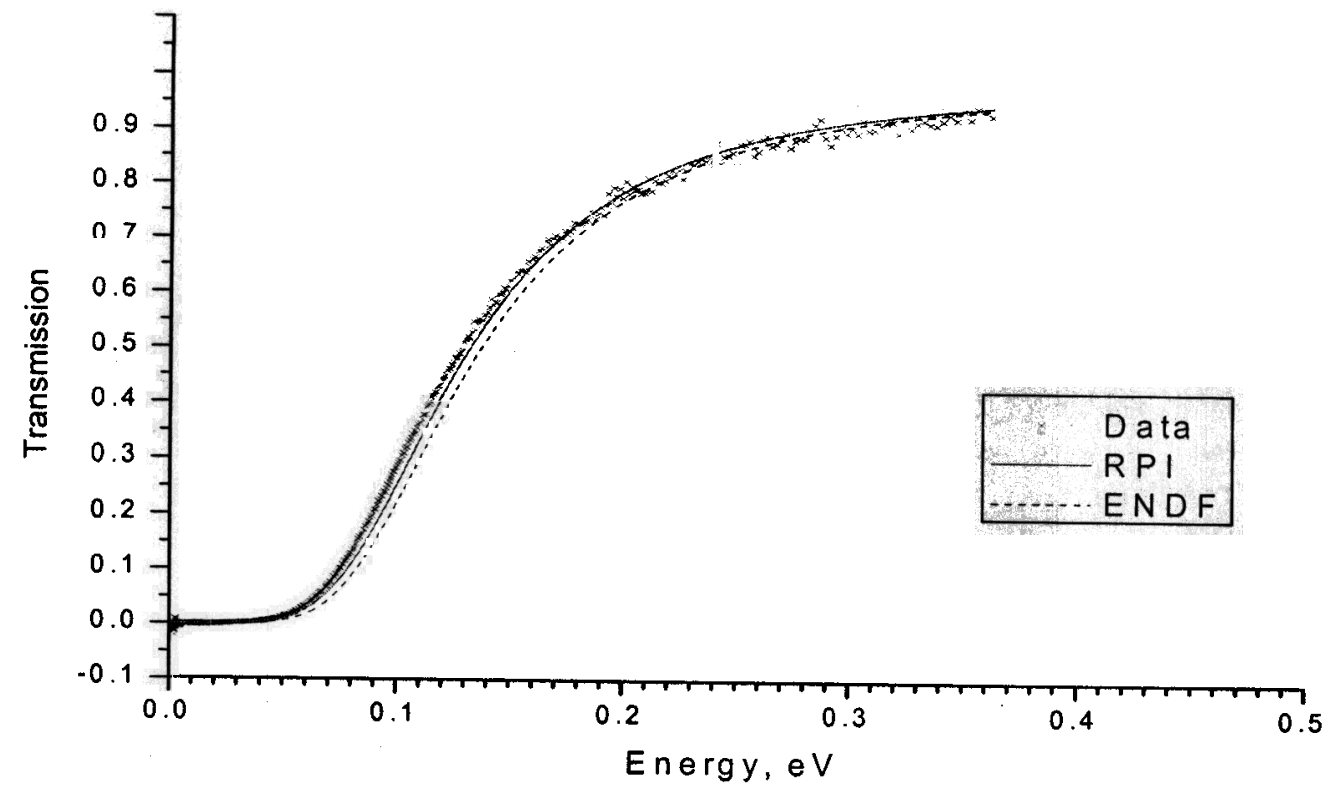

Figure 14- LX-6 liquid Gd thermal transmission (experiment_a) data. The data shown have been corrected for $0.56 \%$ beam leakage past the sample. The RPI fit represents a combined 13-sample SAMMY fit to liquids and metals in transmission and capture. The sample is enriched in ${ }^{157} \mathrm{Gd}$ (see Tables II and III).

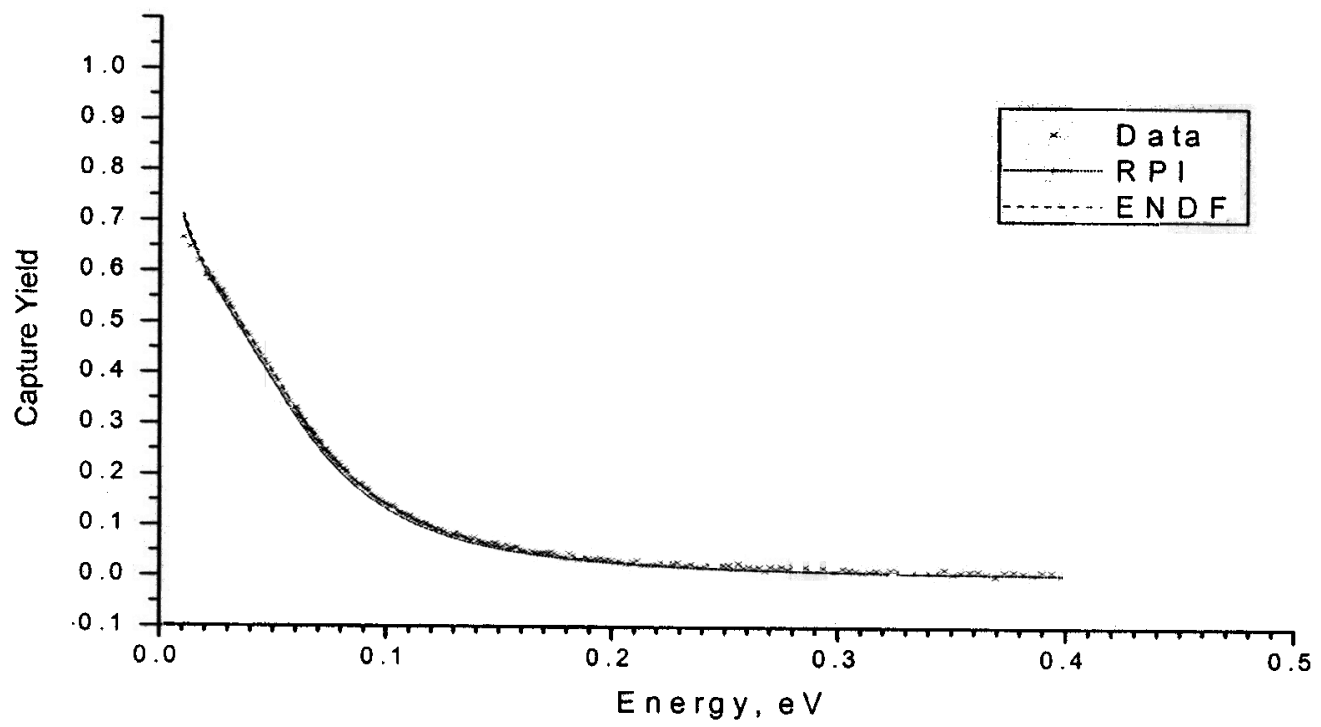

Figure 15- LX-4 liquid Gd thermal capture data. LX-4 is enriched in ${ }^{155} \mathrm{Gd}$. The RPI fit represents a combined 13-sample SAMMY fit to liquids and metals in transmission and capture. Capture normalization is a variable in the fit. The ENDF resonance parameters fit the data better than the RPI parameters, because the combined fit is driven by transmission data and ${ }^{157} \mathrm{Gd}$-absorptiondominated sample data. 


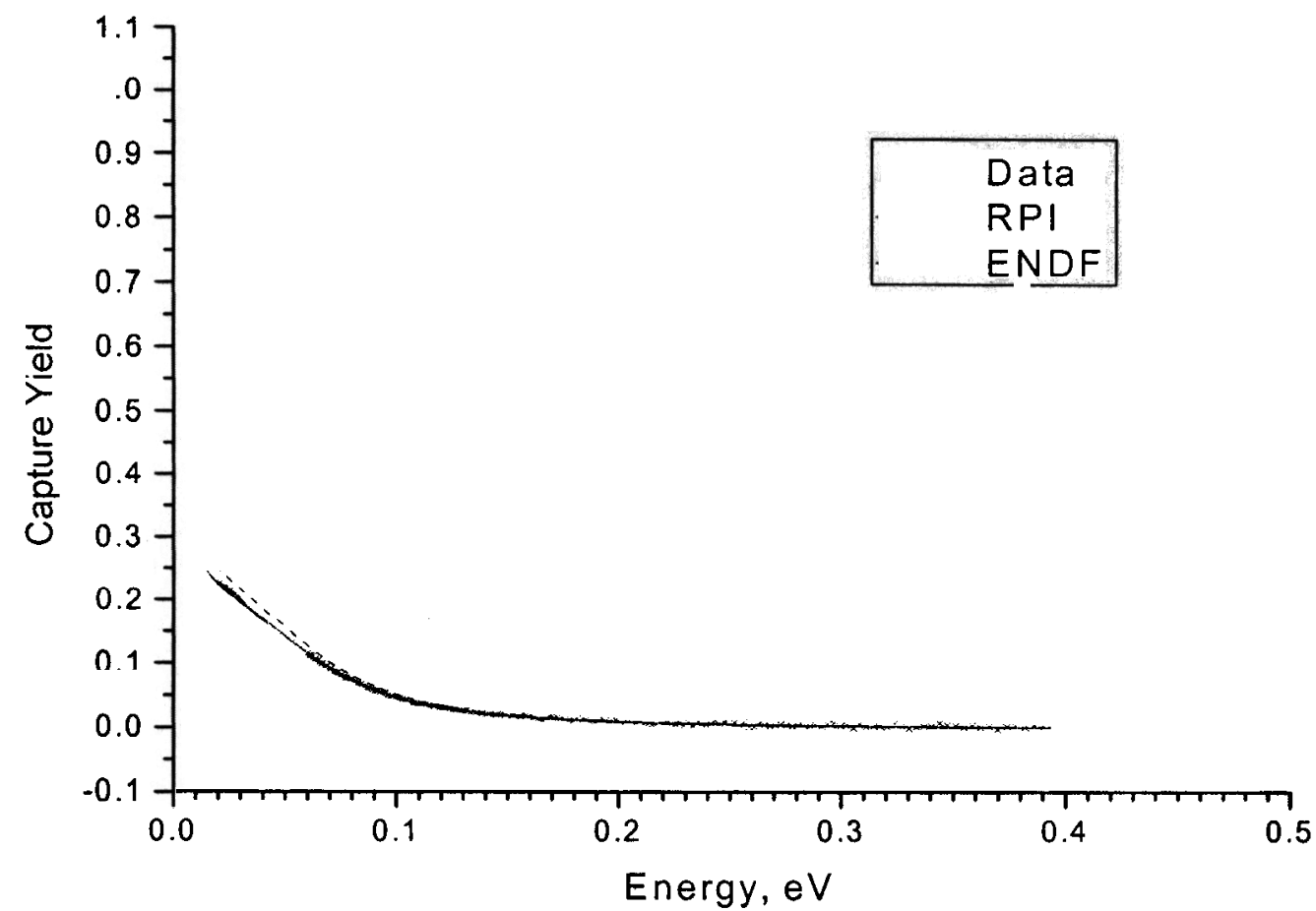

Figure 16- LX-11 liquid Gd thermal capture data. LX-11 is enriched in ${ }^{157}$ Gd. The RPI fit represents a combined 13-sample SAMMY fit to liquids and metals in transmission and capture. Capture normalization is a variable in the fit. 


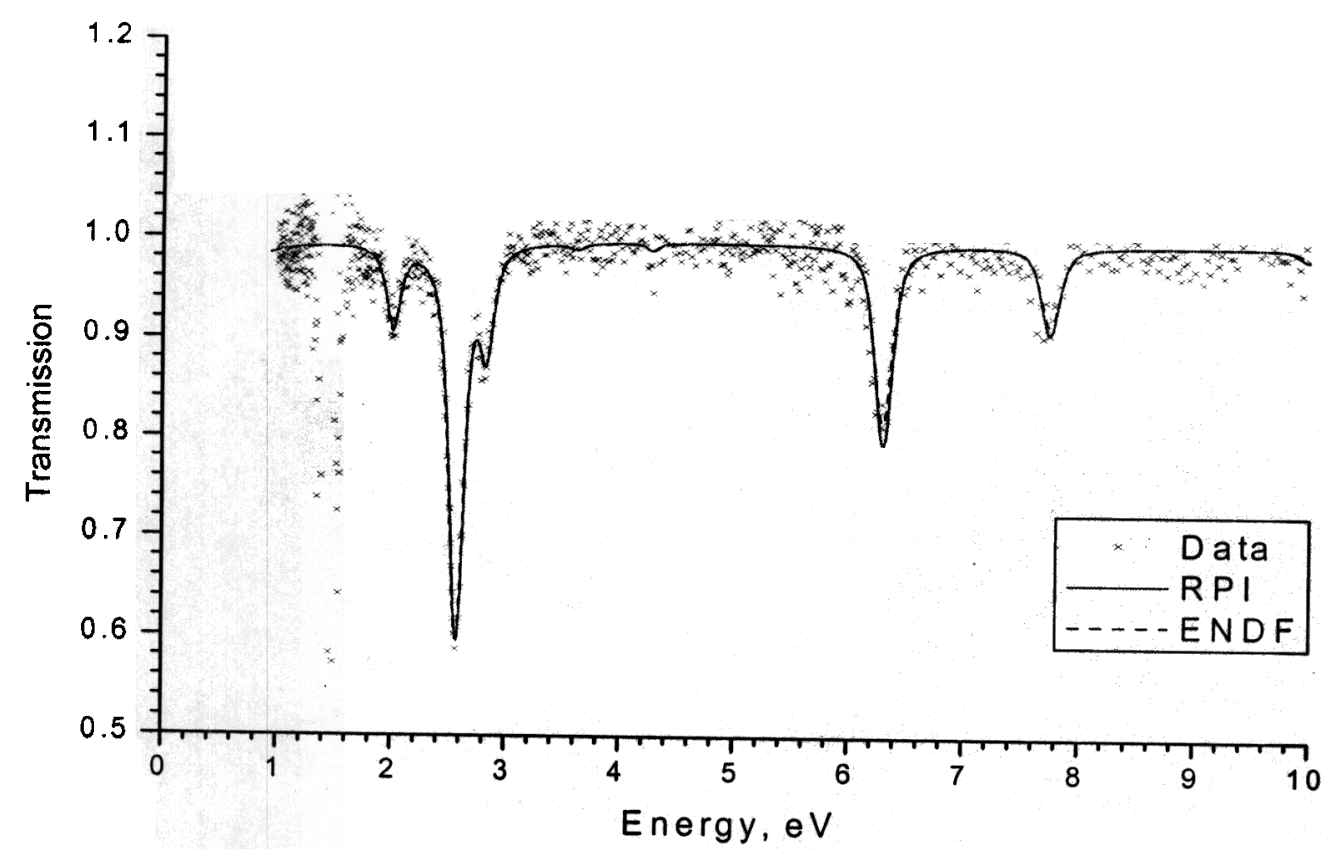

Figure 17-0.127-mm (5-mil) Gd metal thermal transmission data. The RPI fit represents a combined 6-sample SAMMY fit to metal thermal transmission and metal epithermal capture data.

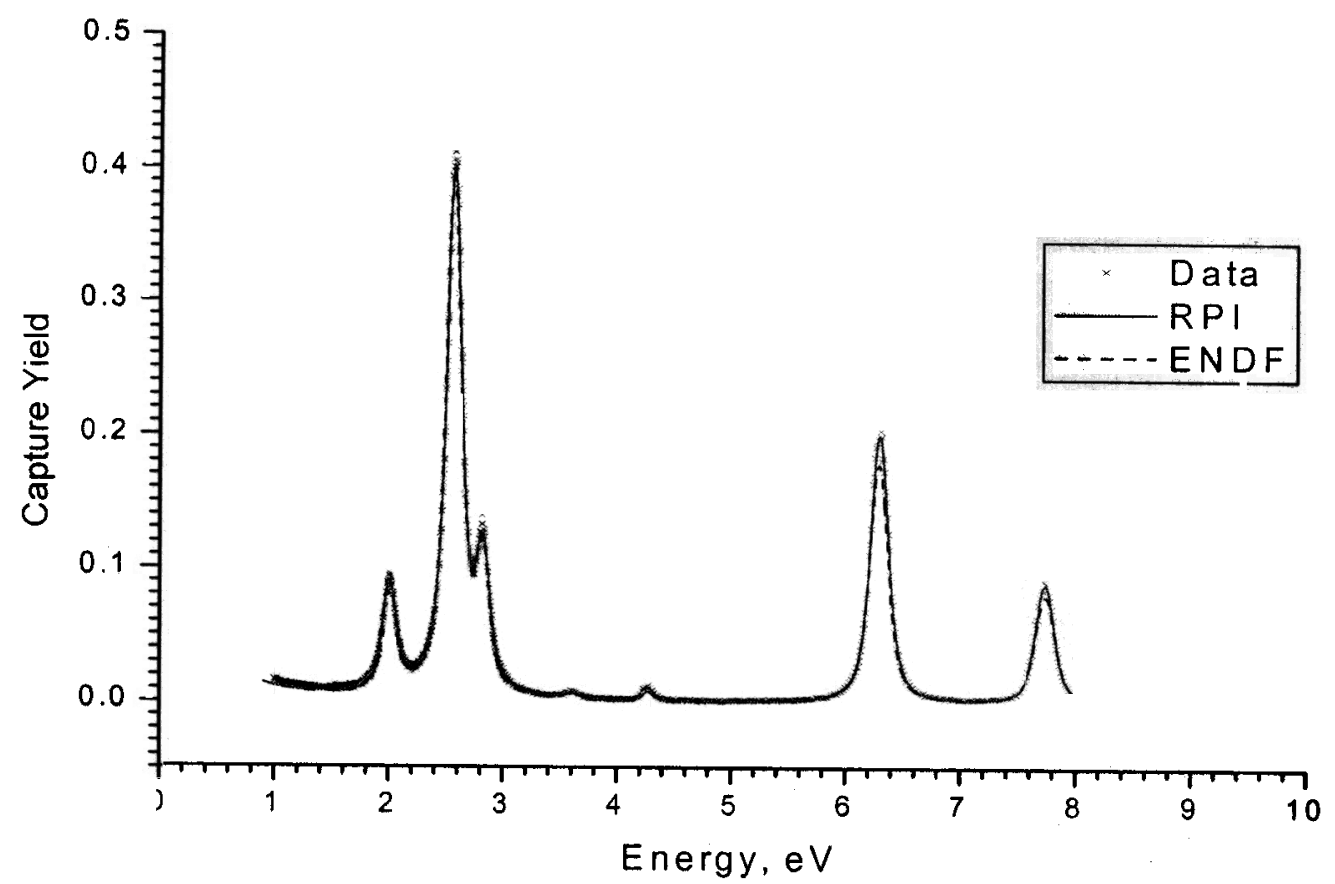

Figure 18- 0.127-mm (5-mil) Gd metal epithermal neutron capture yield data. The RPI fit represents a combined 6-sample SAMMY fit to metal thermal transmission and metal epithermal capture data. 


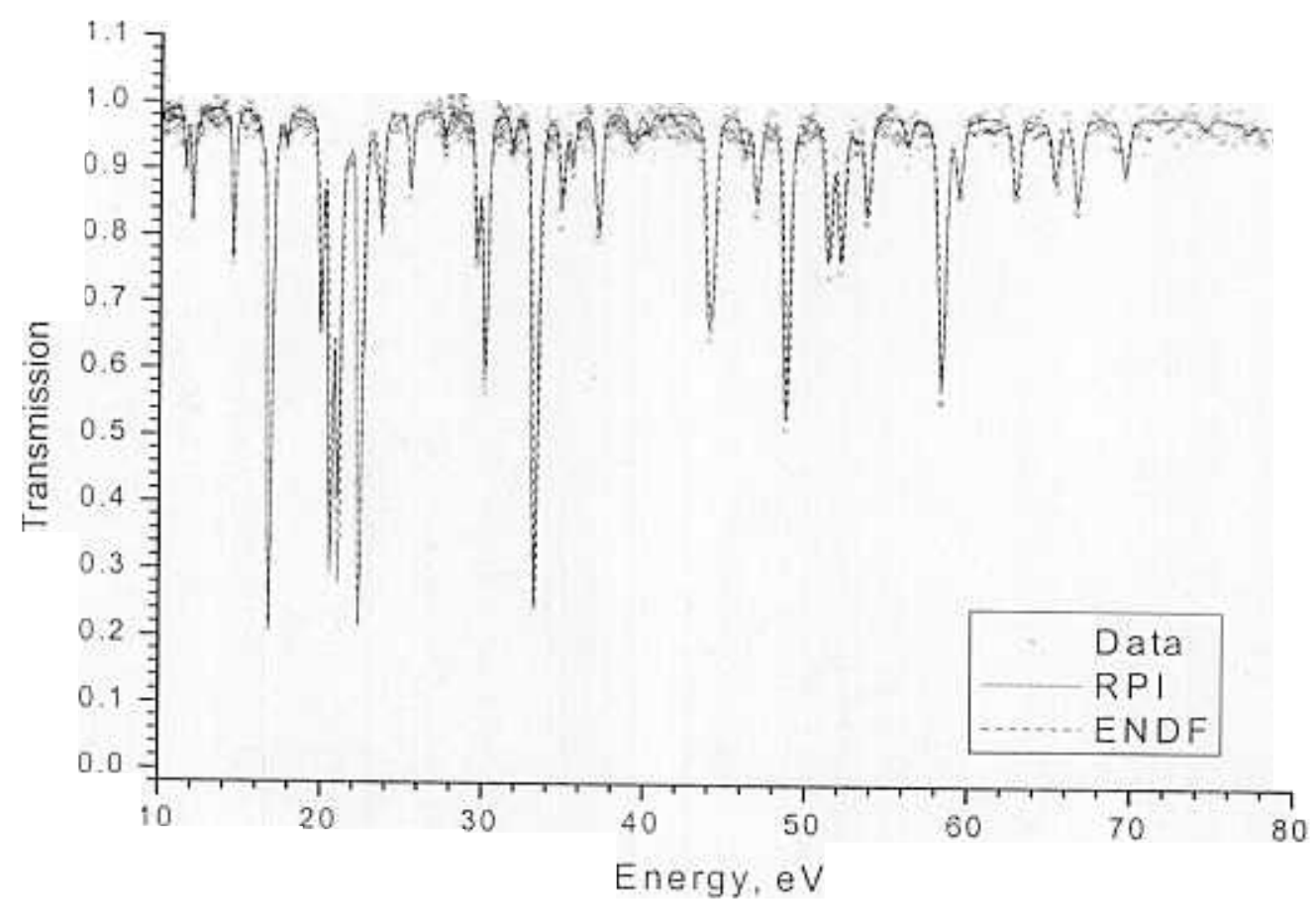

Figure 19-0.508-mm (20-mil) natural metal Gd transmission data in the 10-78 eV region. Data are from the boron-filtered measurement. The RPI fit represents a combined 15-sample SAMMY fit to epithermal metal transmission and capture data.

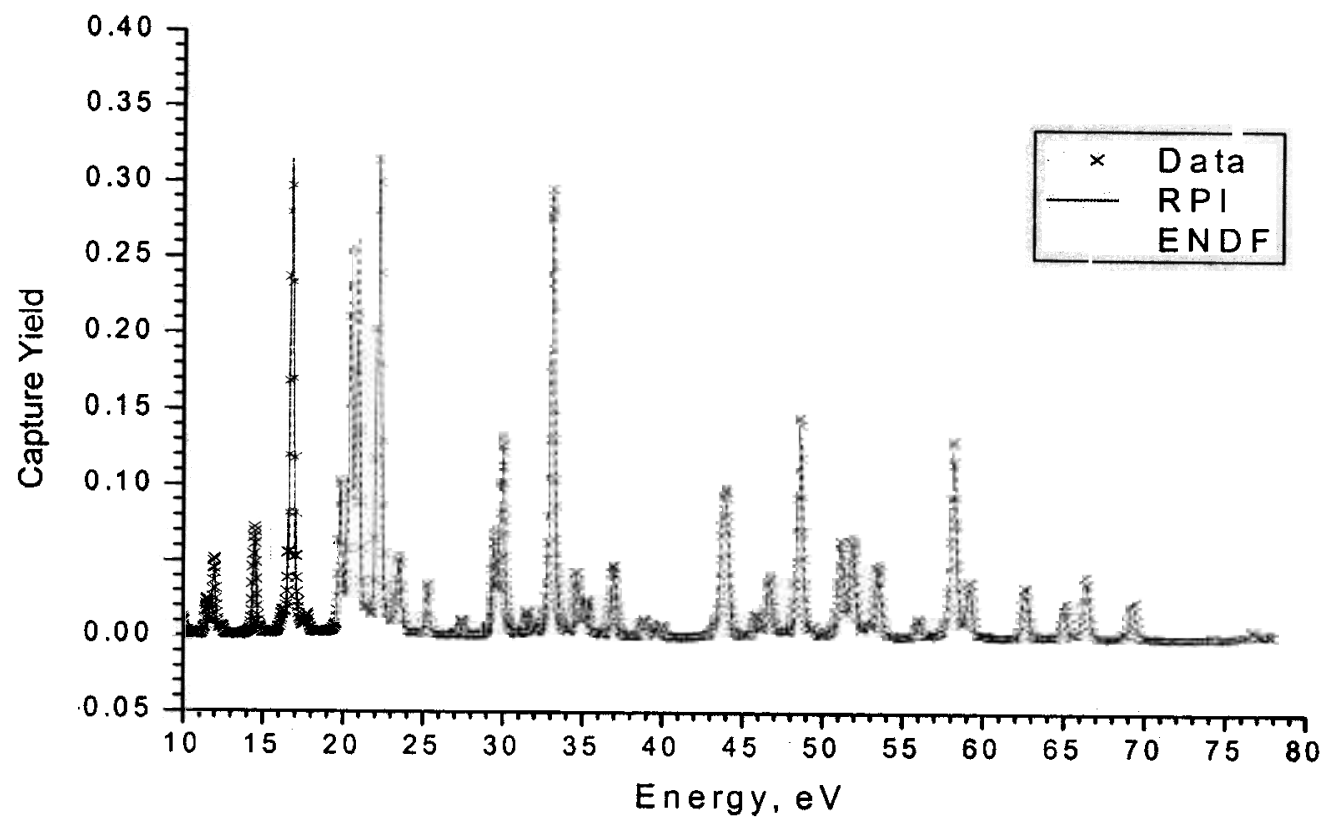

Figure 20- 0.127-mm (5-mil) natural metal Gd neutron capture data in the $10-78 \mathrm{eV}$ region. The RPI fit represents a combined 15-sample SAMMY fit to epithermal metal transmission and capture data. 


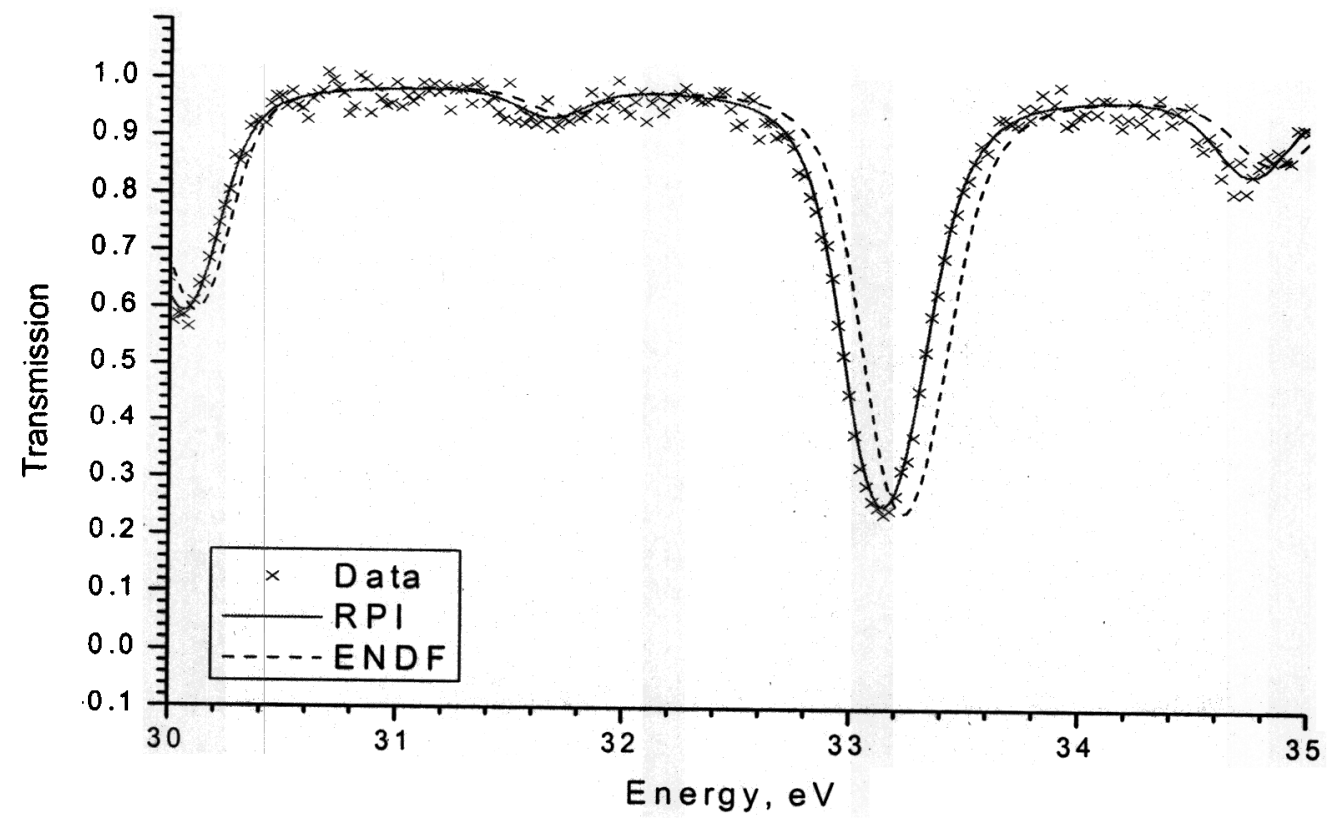

Figure 21- 0.508-mm (20-mil) natural metal Gd transmission data in the 30-35 eV region. Data are from the boron-filtered measurement. The RPI fit represents a combined 15-sample SAMMY fit to epithermal metal transmission and capture data.

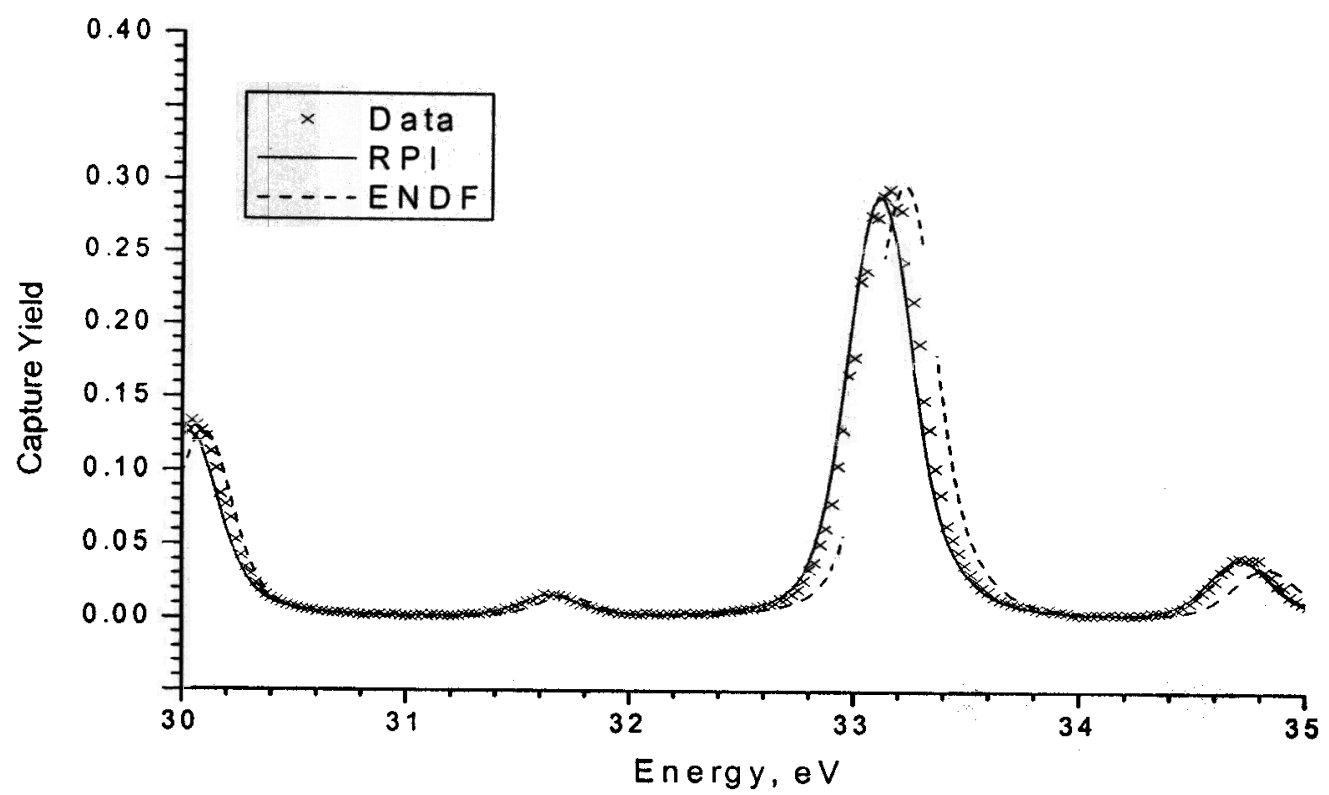

Figure 22 - 0.127-mm (5-mil) natural metal Gd neutron capture data in the 30-35 eV region. The RPI fit represents a combined 15-sample SAMMY fit to epithermal metal transmission and capture data. There is an energy mismatch between transmission (Figure 21) and capture data due to independent measurements of flight path and zero time. The offset is $\approx 0.03 \mathrm{eV}$, (see $\Delta \mathrm{E}_{0}$ in Table VII for the $33.14 \mathrm{eV}$ resonance). The offset does not affect the SAMMY fit. 


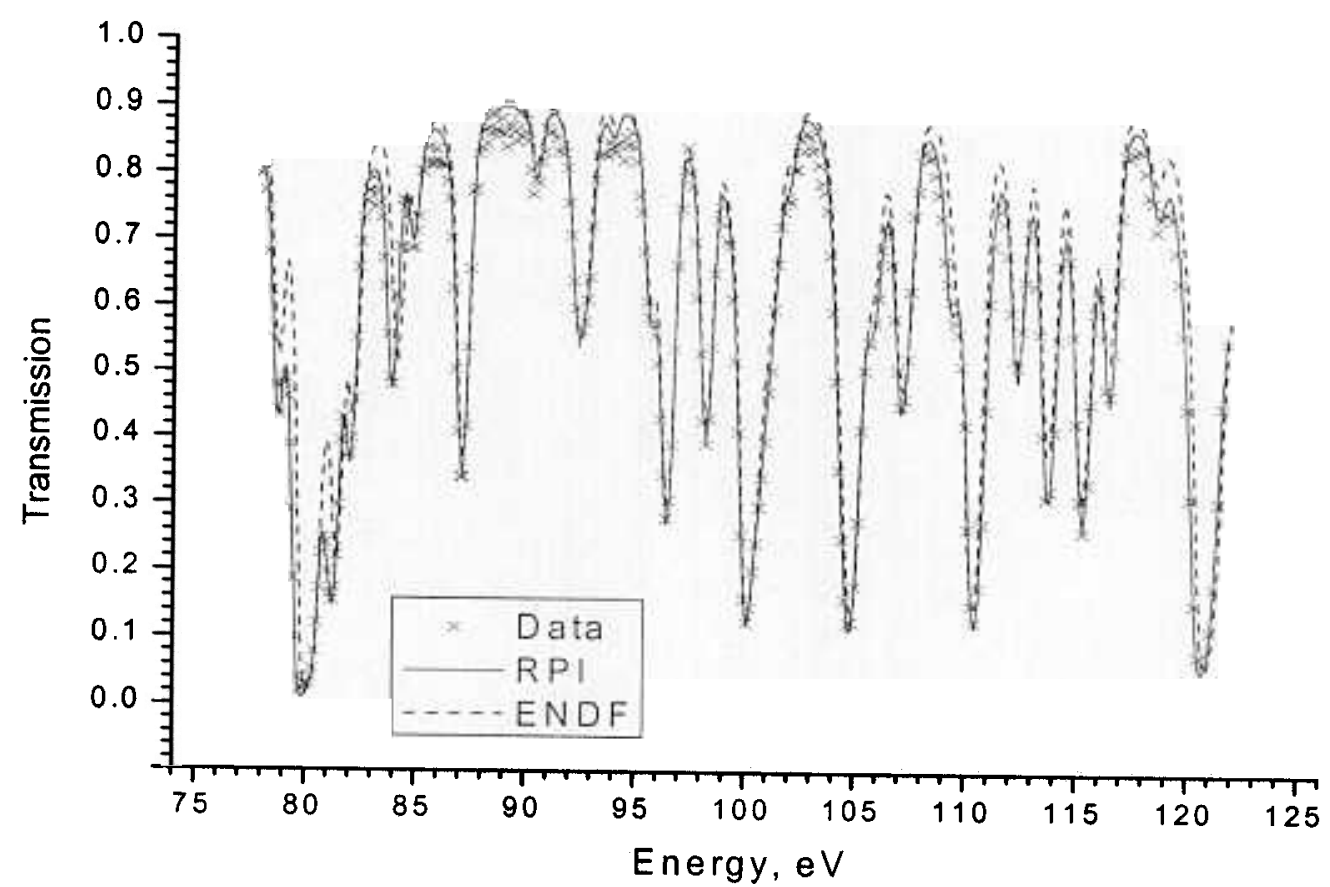

Figure 23- 5.08-mm (200-mil) metal Gd epithermal transmission data. The RPI fit represents a combined 15-sample SAMMY fit to epithermal metal transmission and capture data.

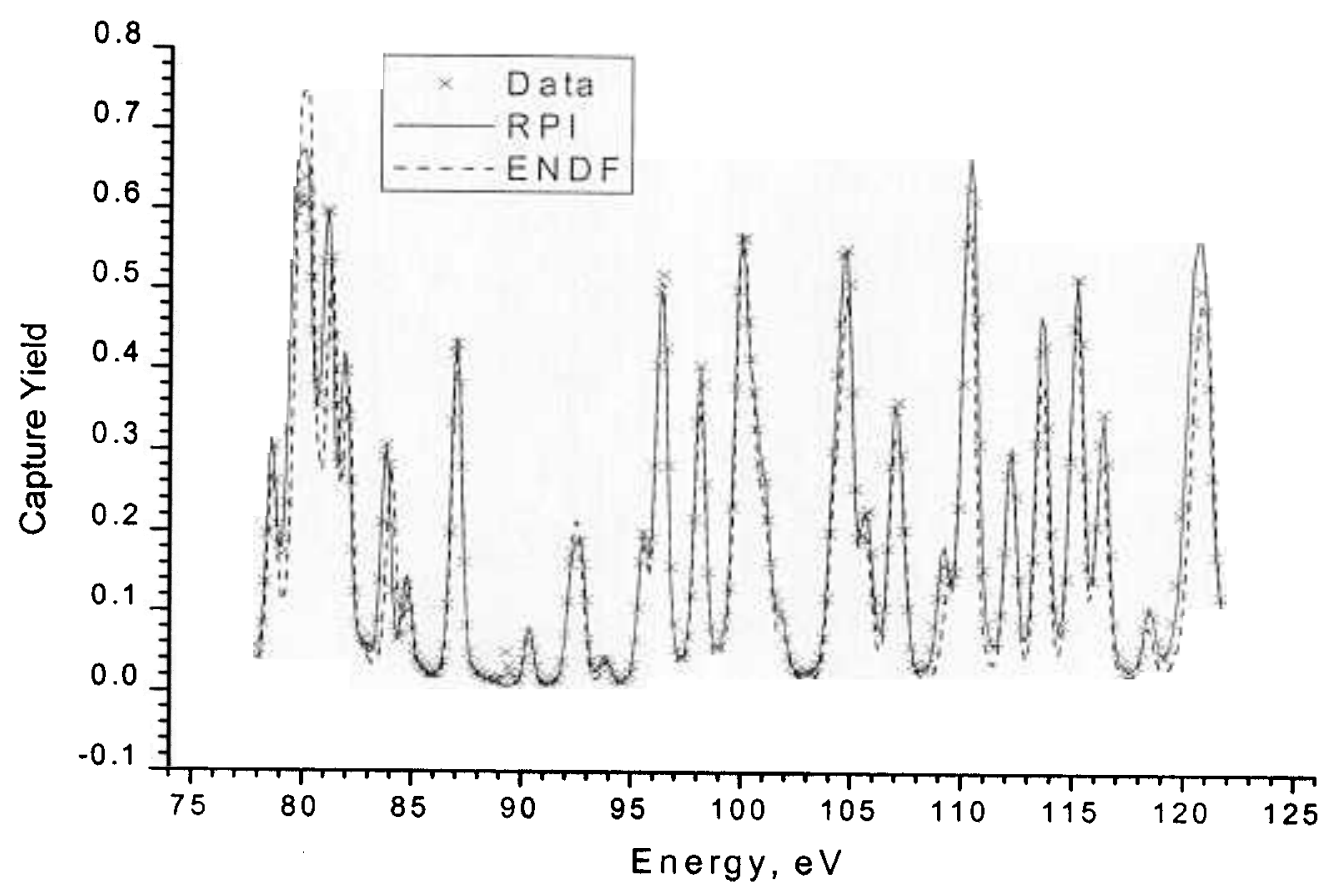

Figure 24- 2.54-mm (100-mil) metal Gd epithermal capture data. The RPI fit represents a combined 15-sample SAMMY fit to epithermal metal transmission and capture data. 


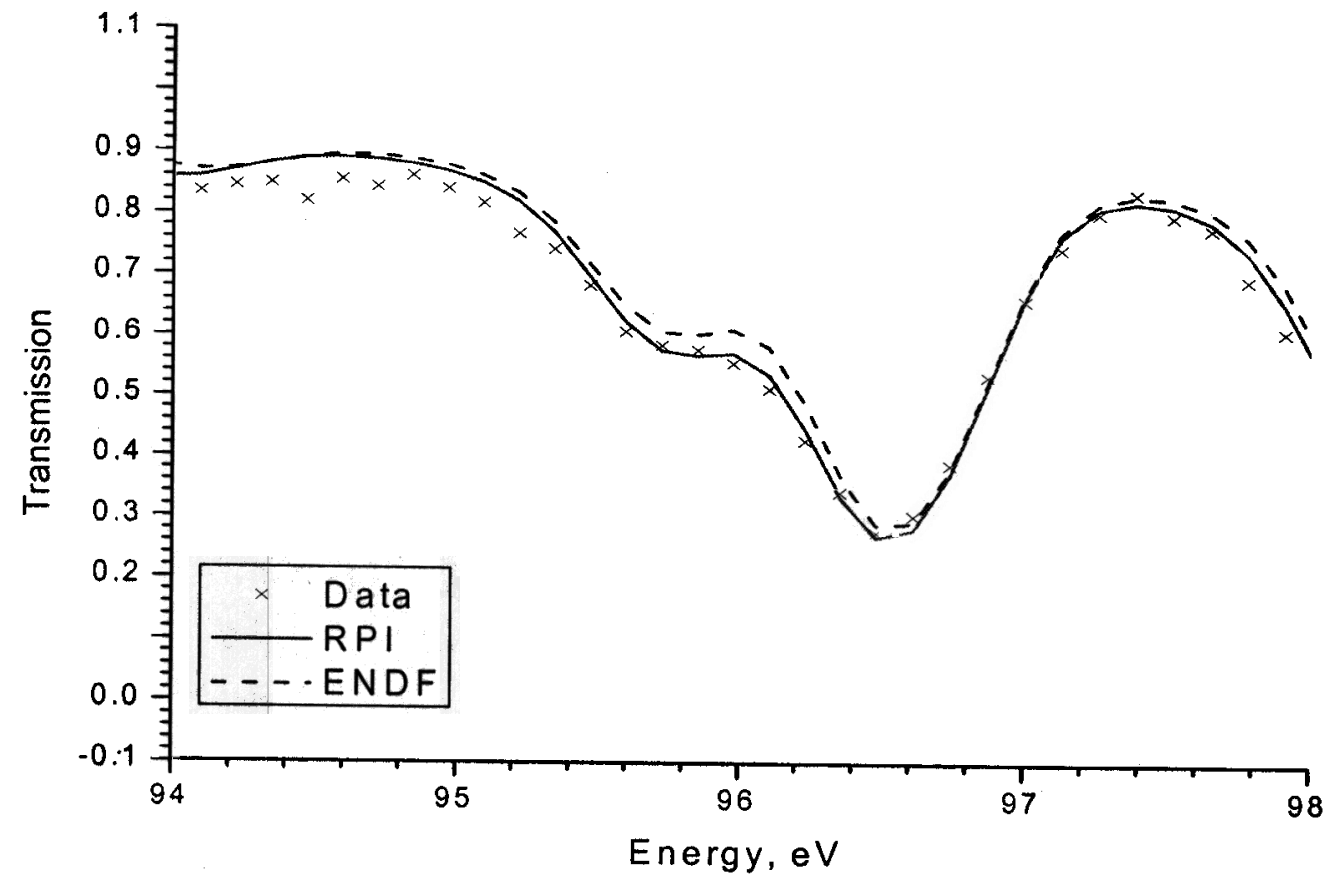

Figure 25- 5.08-mm (200-mil) metal Gd epithermal transmission data in the $96 \mathrm{eV}$ region. The RPI fit represents a combined 15-sample SAMMY fit to epithermal metal transmission and capture data.

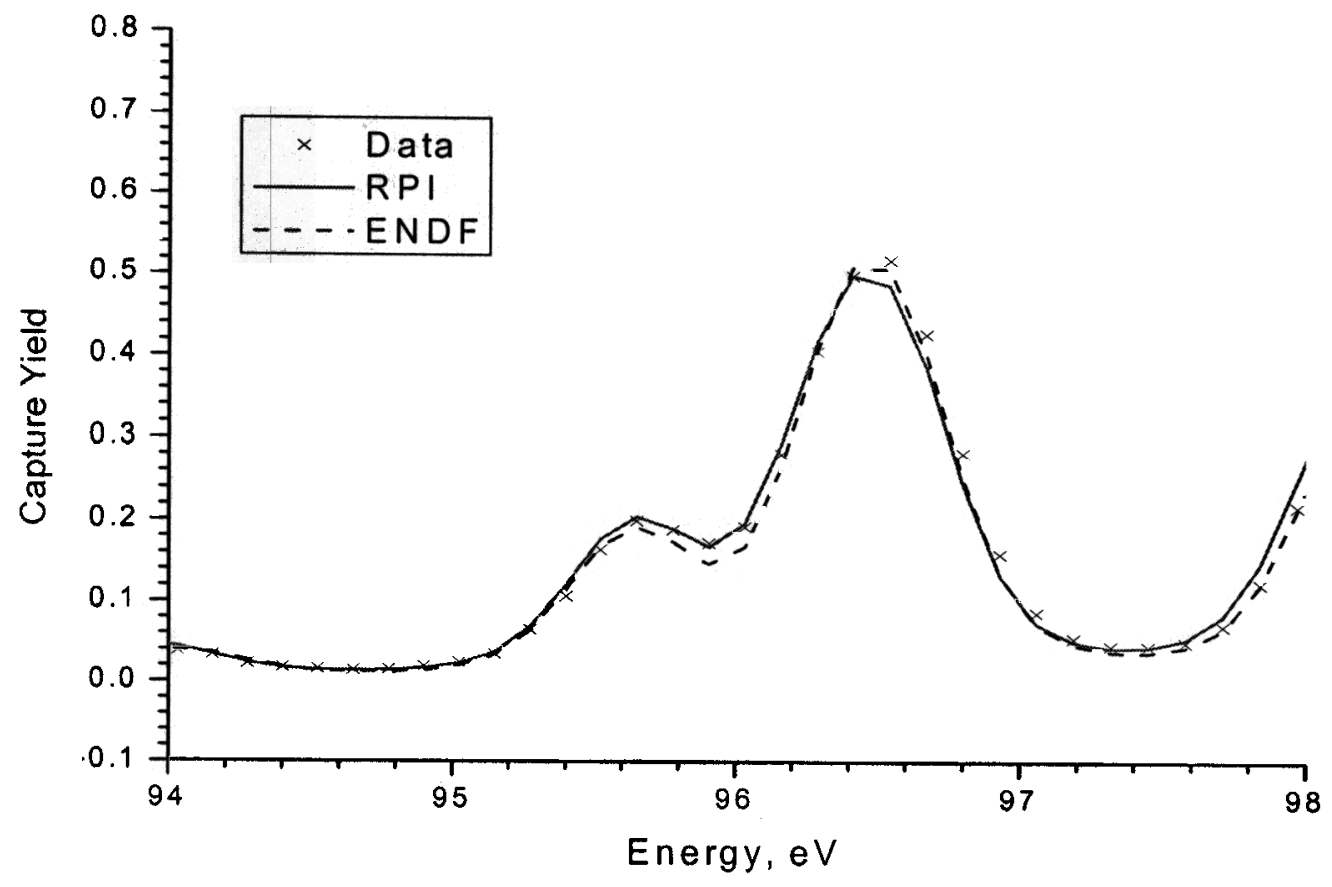

Figure 26- 2.54-mm (100-mil) metal Gd epithermal capture data in the $96 \mathrm{eV}$ region. The RPI fit represents a combined 15-sample SAMMY fit to epithermal metal transmission and capture data. 


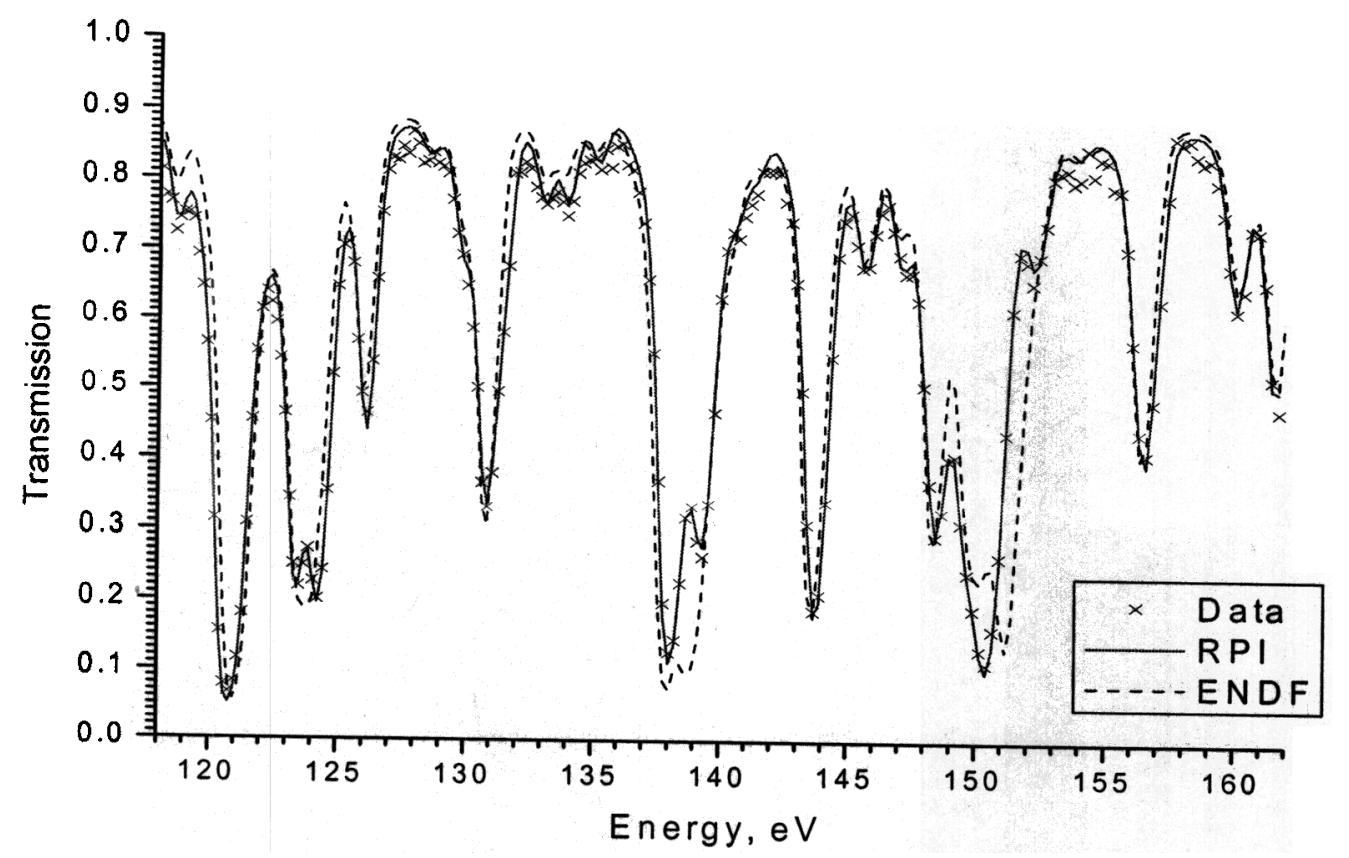

Figure 27 - 5.08-mm $(200-\mathrm{mil})$ metal Gd epithermal transmission data. The RPI fit represents a combined 15-sample SAMMY fit to epithermal metal transmission and capture data.

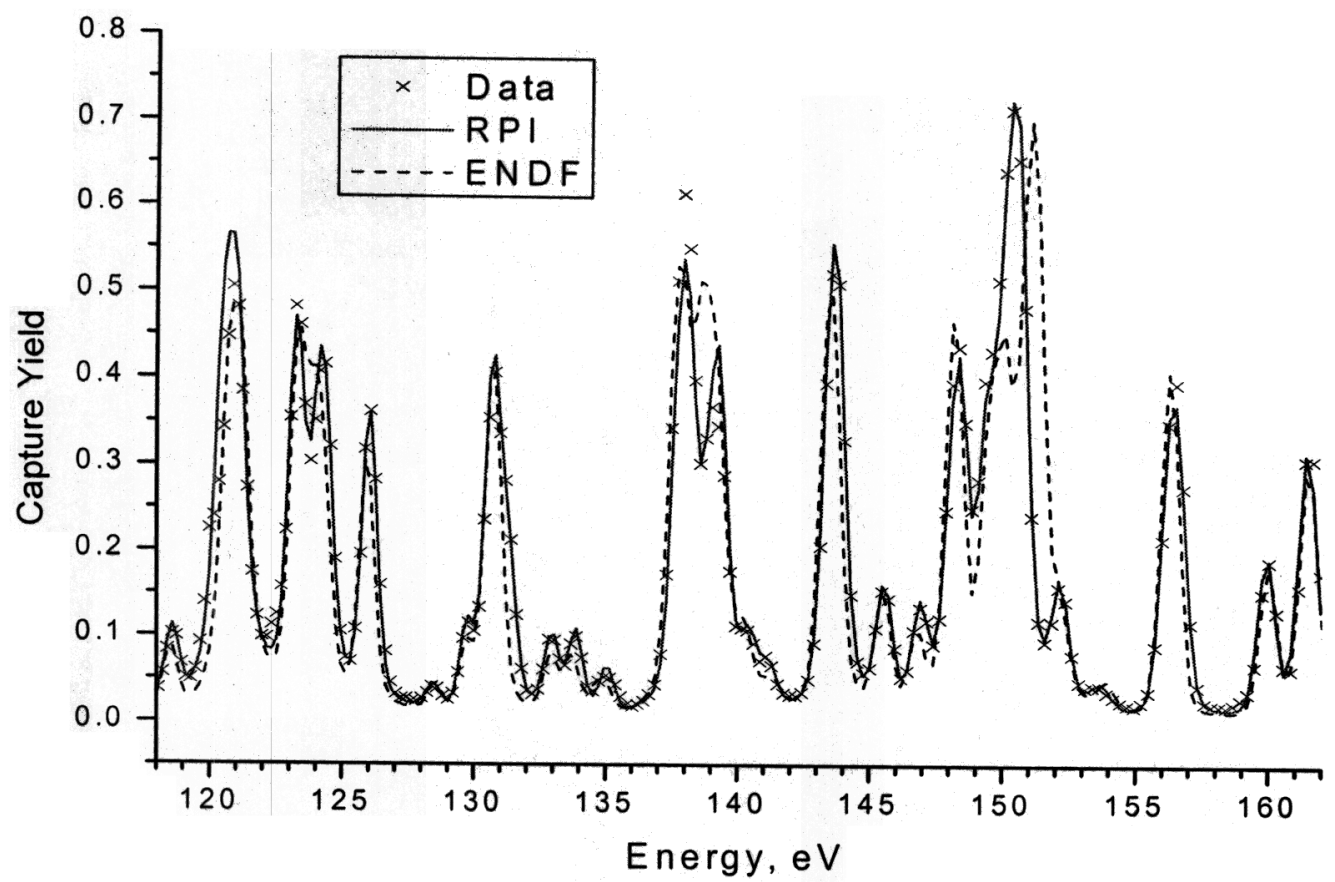

Figure 28 - 2.54-mm (100-mil) metal Gd epithermal capture data. The RPI fit represents a combined 15-sample SAMMY fit to epithermal metal transmission and capture data. 


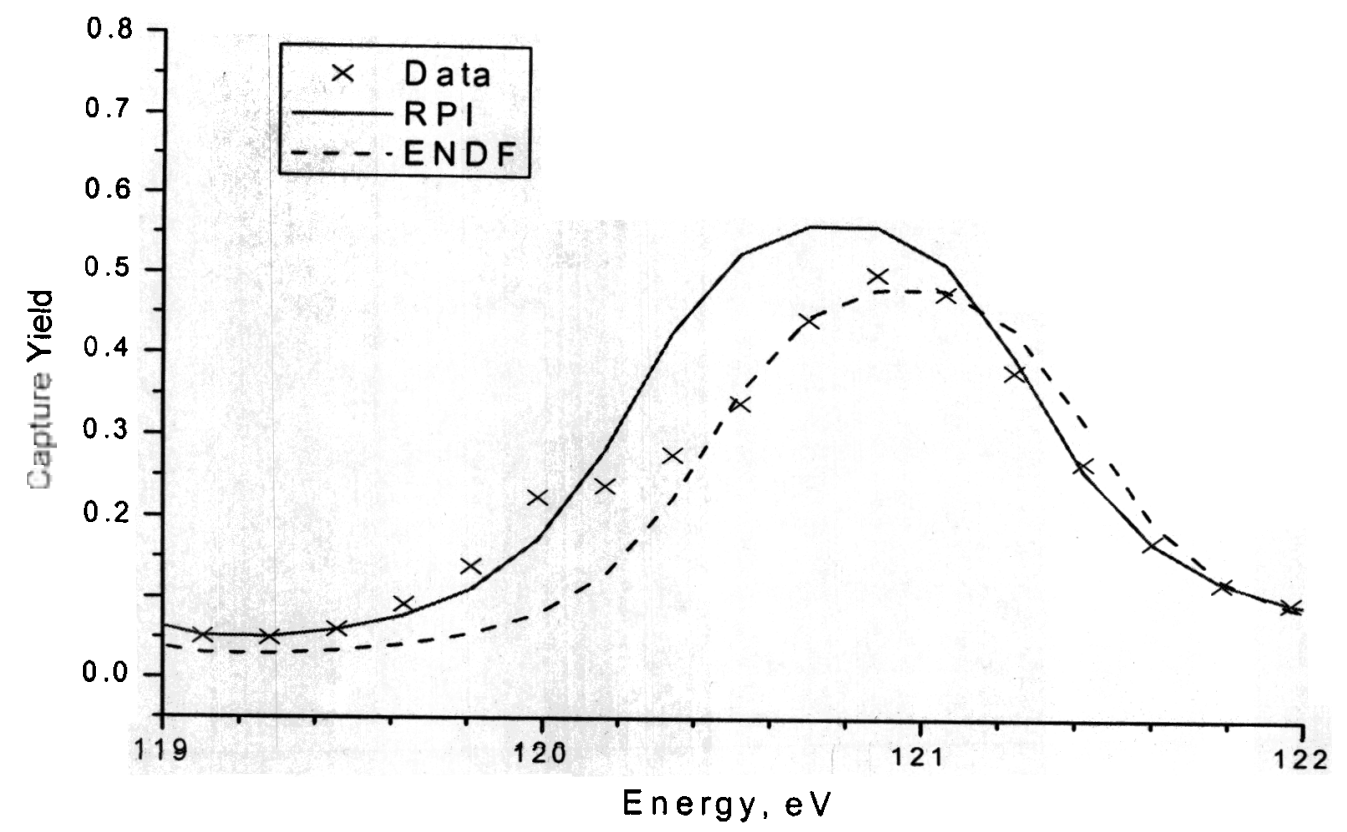

Figure 29 - Final SAMMY fit to $2.54-\mathrm{mm}(100-\mathrm{mil})$ metal capture data showing a single resonance at $120 \mathrm{eV}$. Neither the RPI parameters nor the ENDF parameters fit the data. Attempts to fit the resonance as a doublet were unsuccessful (see Figure 30). The RPI fit represents a combined 15sample SAMMY fit to epithermal metal transmission and capture data.

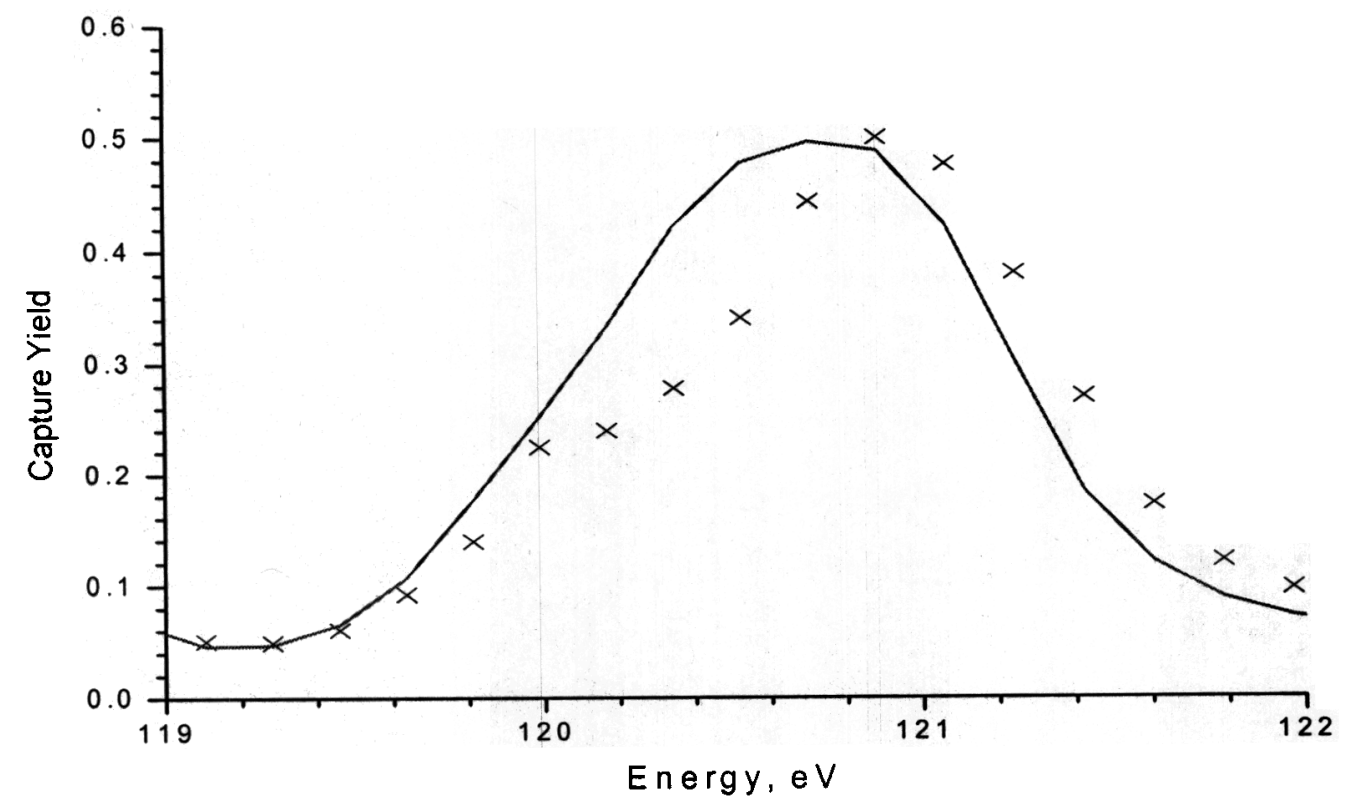

Figure 30 - An attempt to fit the $120 \mathrm{eV}$ region as a doublet. A small 'shoulder' resonance was added at $120.0 \mathrm{eV}$. The fit did not provide a compelling argument to add the 'shoulder' resonance to the final set of resonance parameters. 


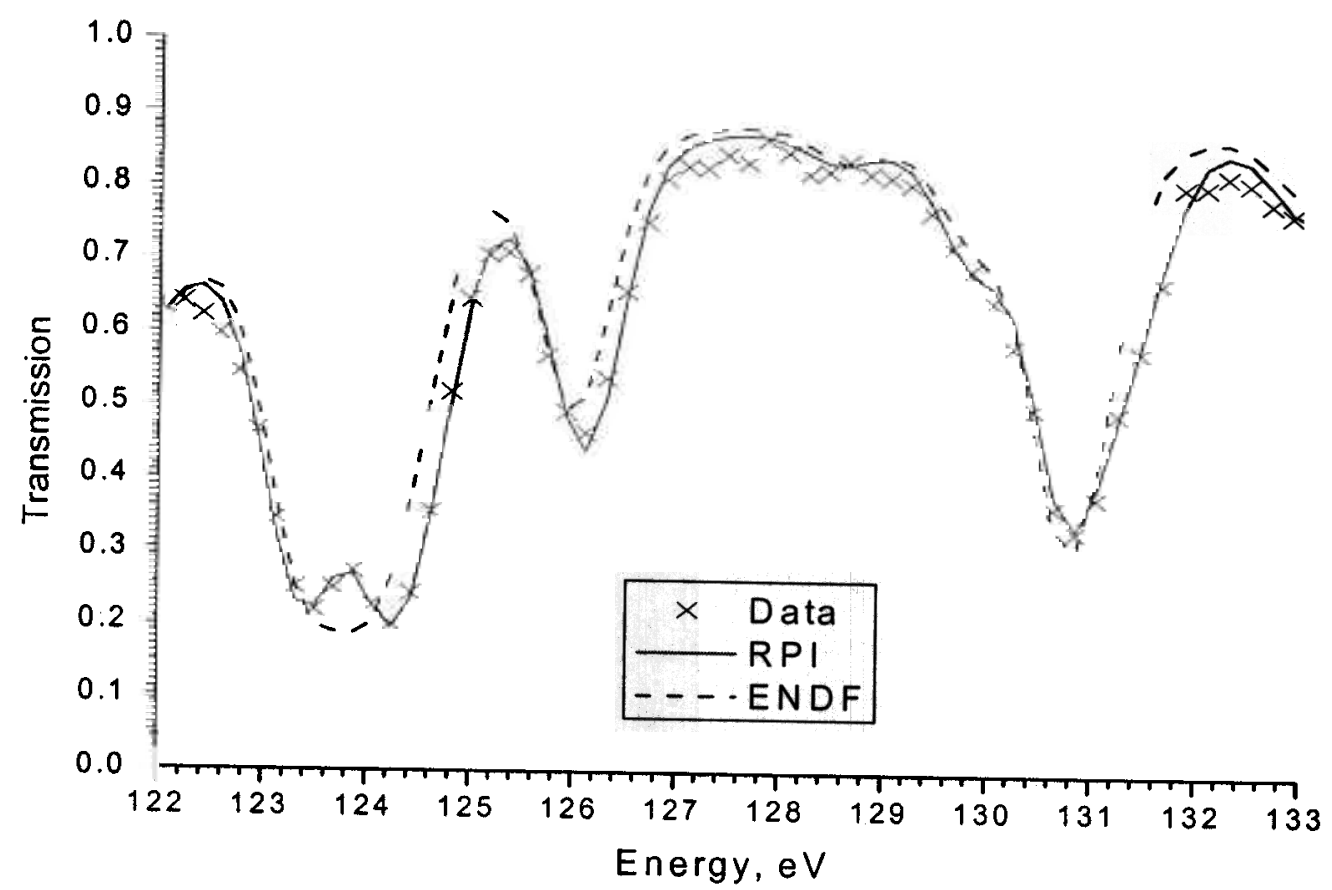

Figure 31 - 5.08-mm (200-mil) metal Gd epithermal transmission data in the $130 \mathrm{eV}$ region. The RPI fit represents a combined 15-sample SAMMY fit to epithermal metal transmission and capture data.

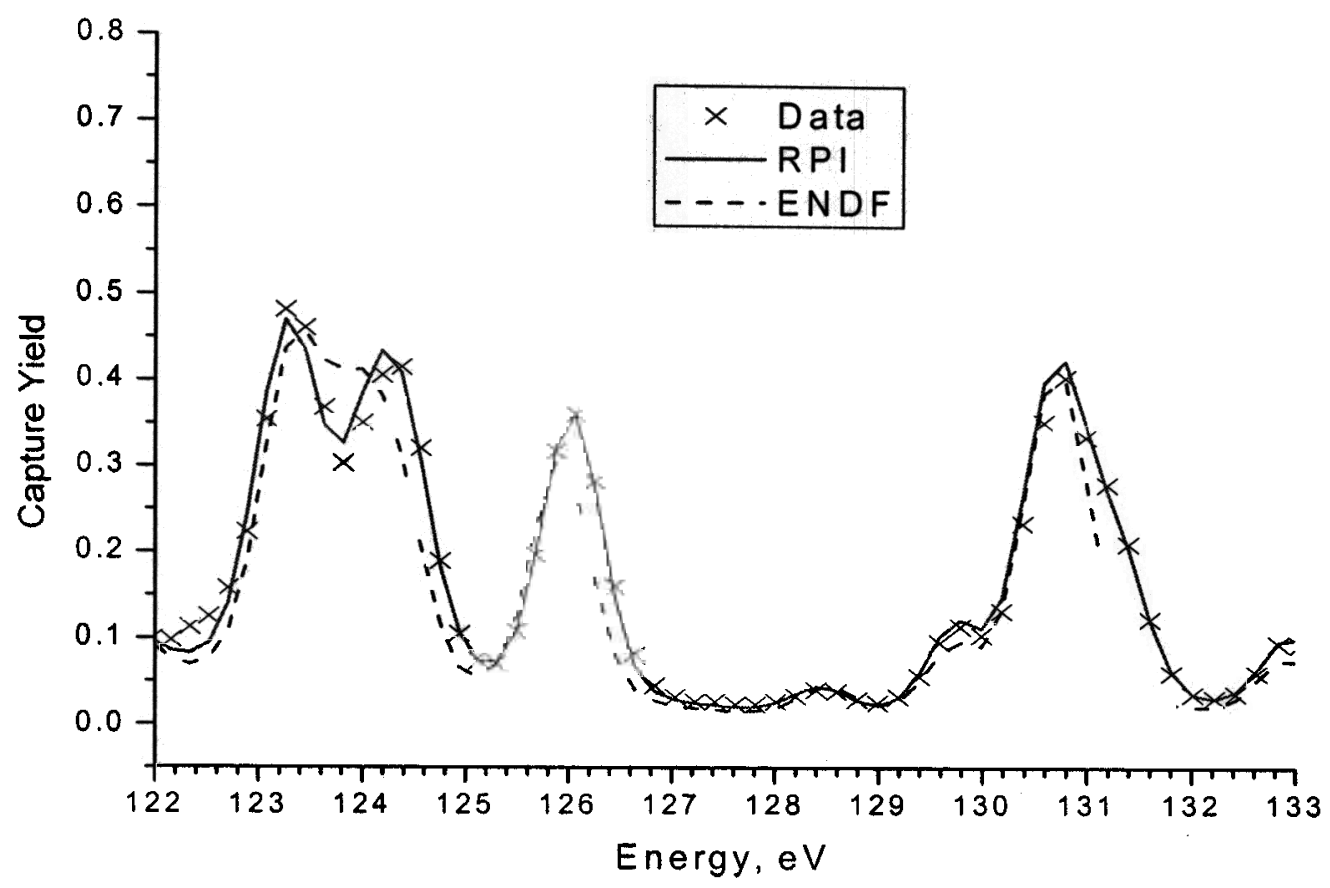

Figure 32 - 2.54-mm (100-mil) metal Gd epithermal capture data in the $130 \mathrm{eV}$ region. The RPI fit represents a combined 15-sample SAMMY fit to epithermal metal transmission and capture data. 


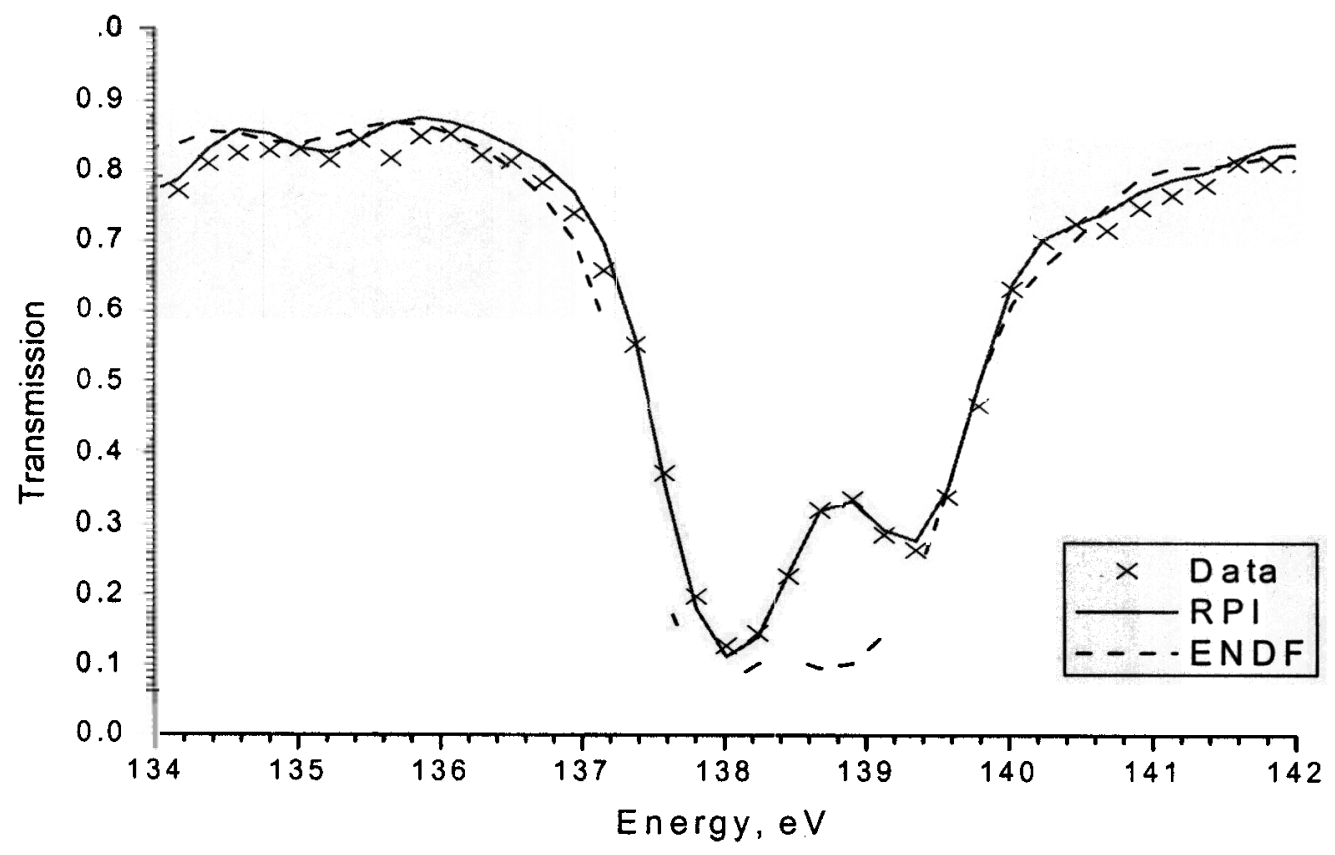

Figure 33 - 5.08-mm (200-mil) metal Gd epithermal transmission data in the $134-142 \mathrm{eV}$ region. The RPI fit represents a combined 15-sample SAMMY fit to epithermal metal transmission and capture data.

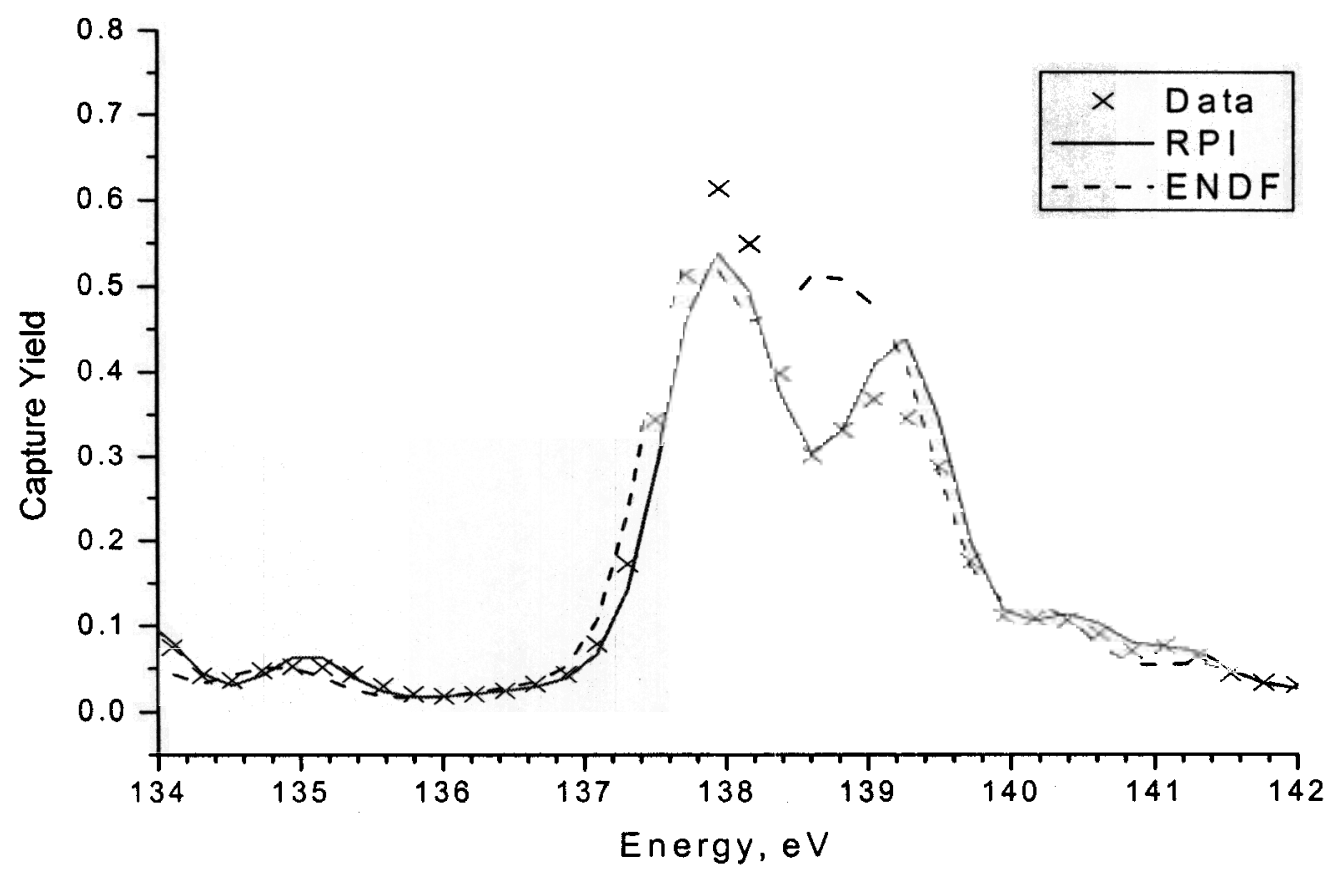

Figure 34 - 2.54-mm (100-mil) metal Gd epithermal capture data in the 134-142 eV region. The RPI fit represents a combined 15-sample SAMMY fit to epithermal metal transmission and capture data. 


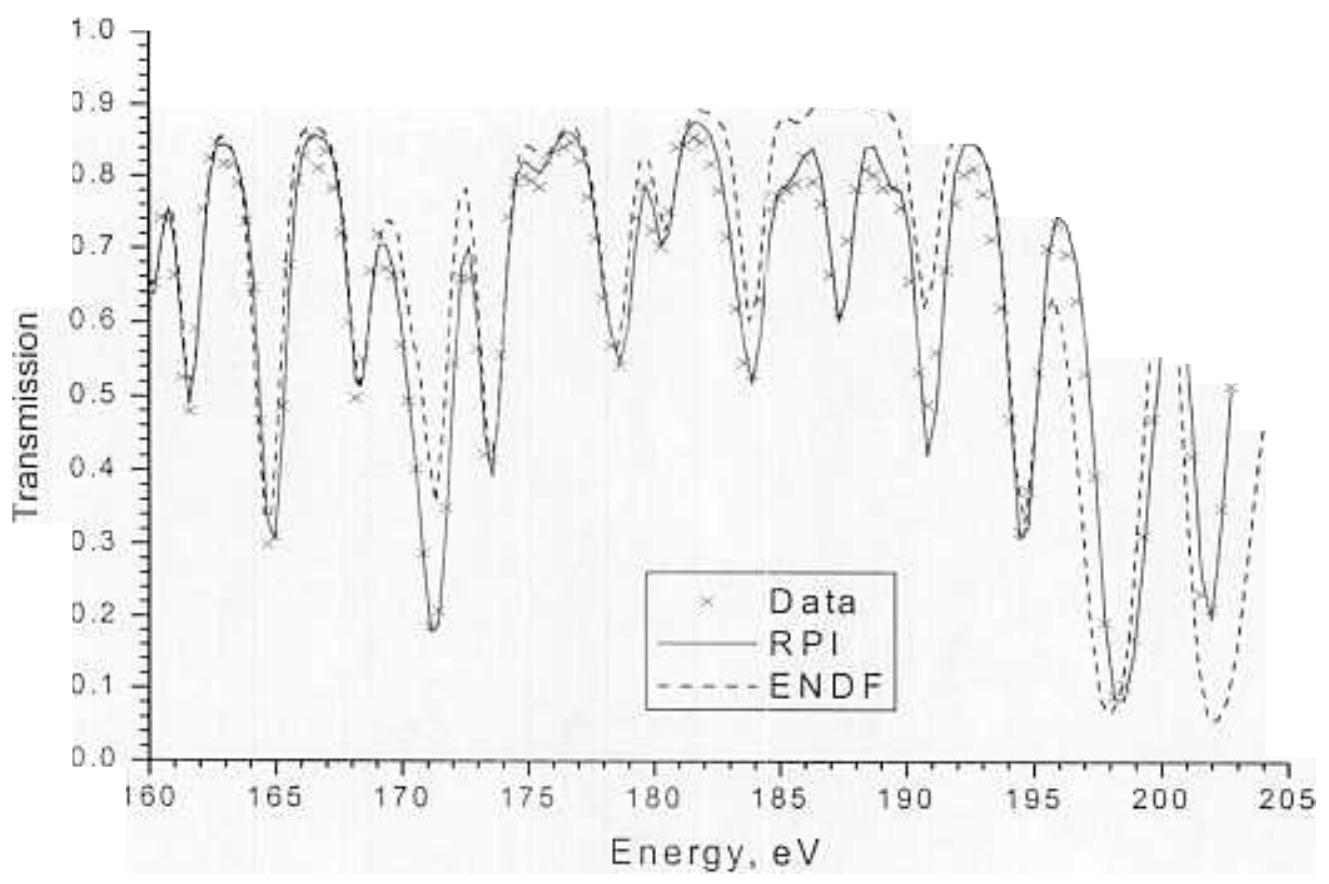

Figure 35 - 5.08-mm (200-mil) metal Gd epithermal transmission data. The RPI fit represents a combined 15-sample SAMMY fit to epithermal metal transmission and capture data.

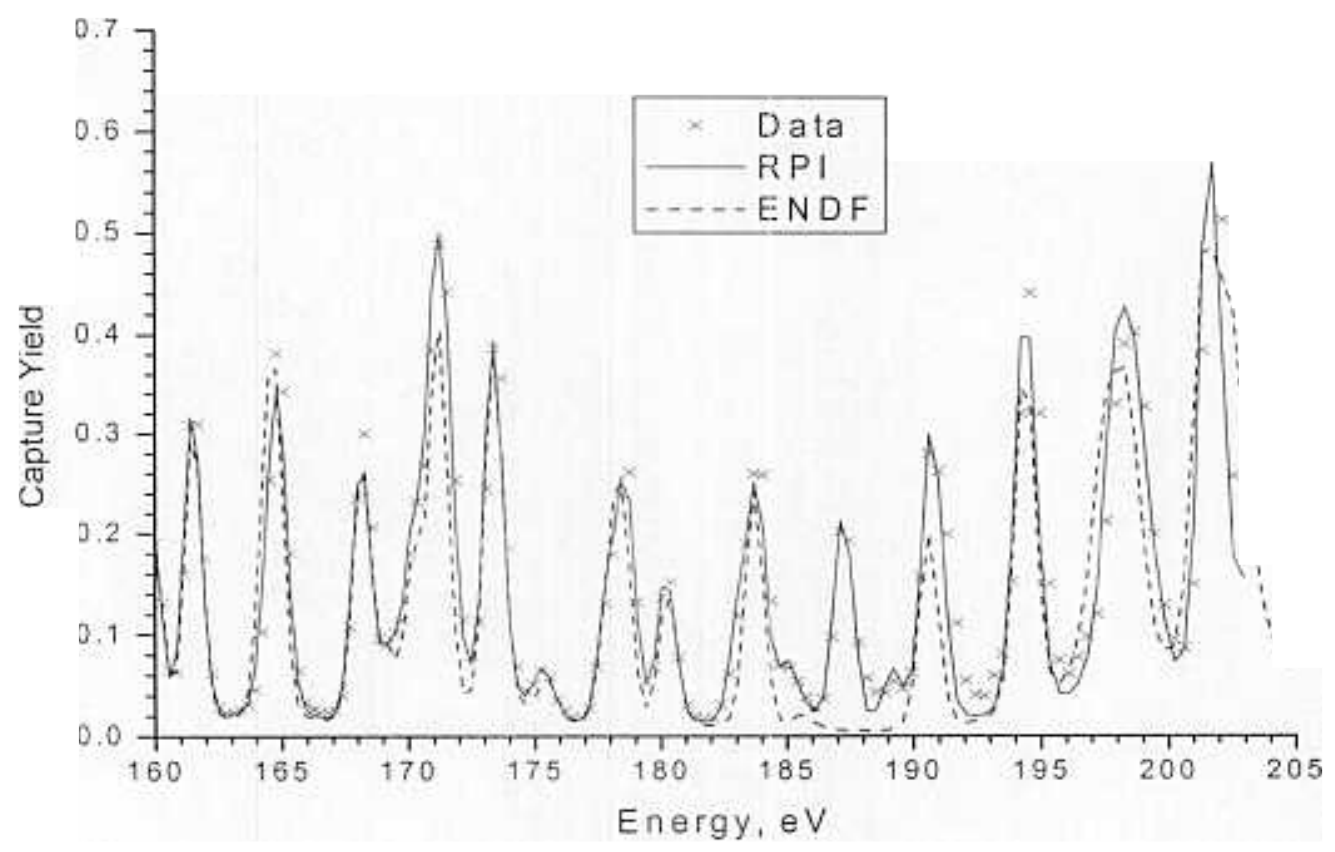

Figure 36 - 2.54-mm (100-mil) metal Gd epithermal capture data. The RPI fit represents a combined 15-sample SAMMY fit to epithermal metal transmission and capture data. 


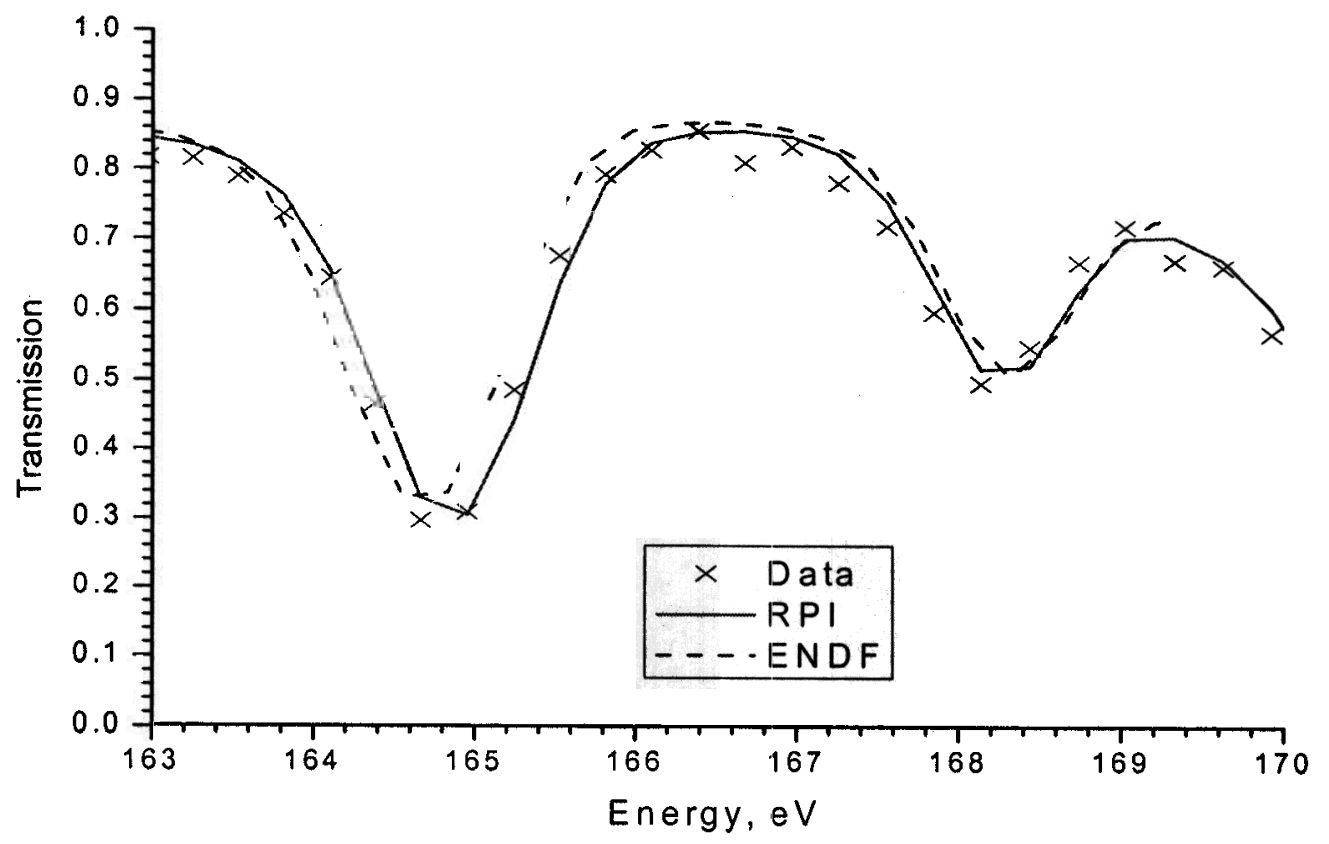

Figure 37 - 5.08-mm (200-mil) metal Gd epithermal transmission data in the $166 \mathrm{eV}$ region. The RPI fit represents a combined 15-sample SAMMY fit to epithermal metal transmission and capture data.

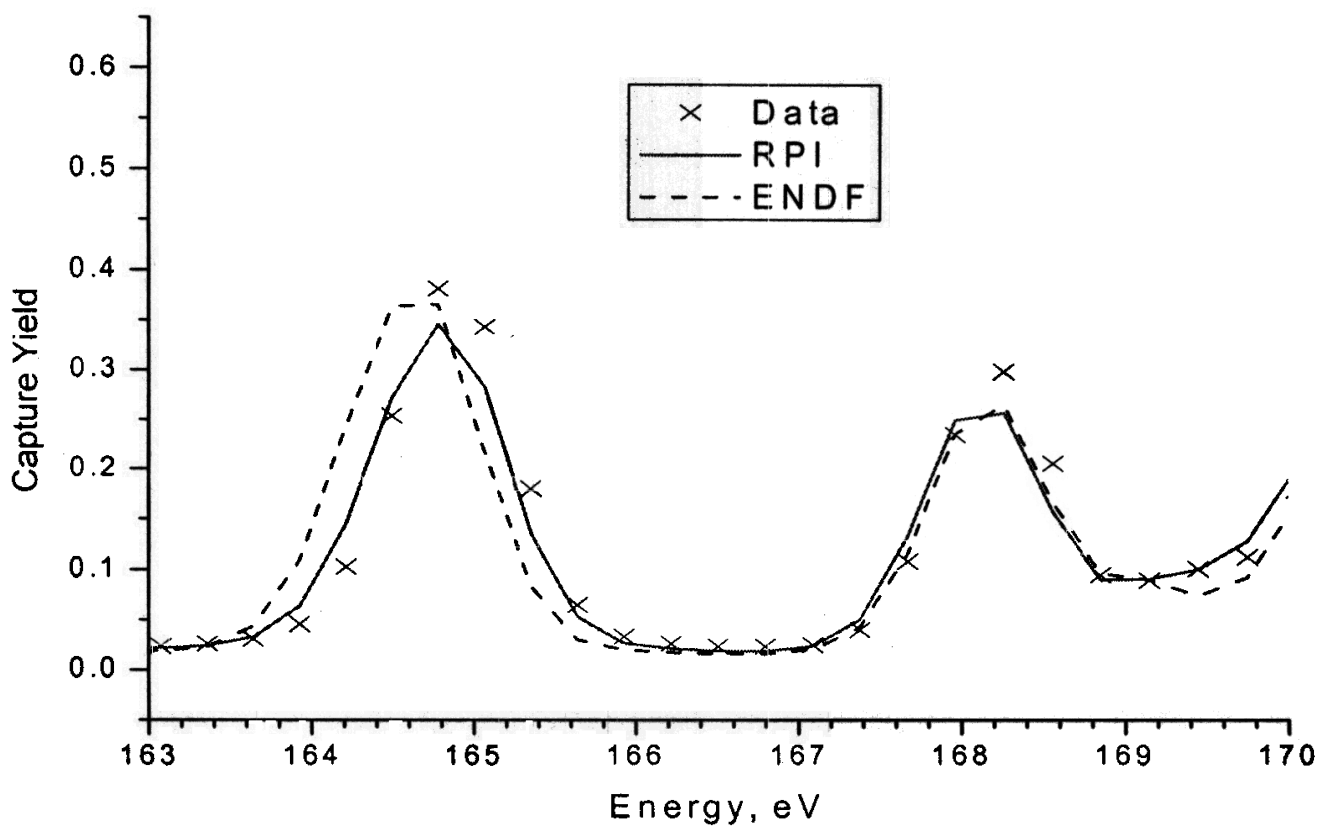

Figure 38 - 2.54-mm (100-mil) metal Gd epithermal capture data in the $166 \mathrm{eV}$ region. The RPI fit represents a combined 15-sample SAMMY fit to epithermal metal transmission and capture data. 


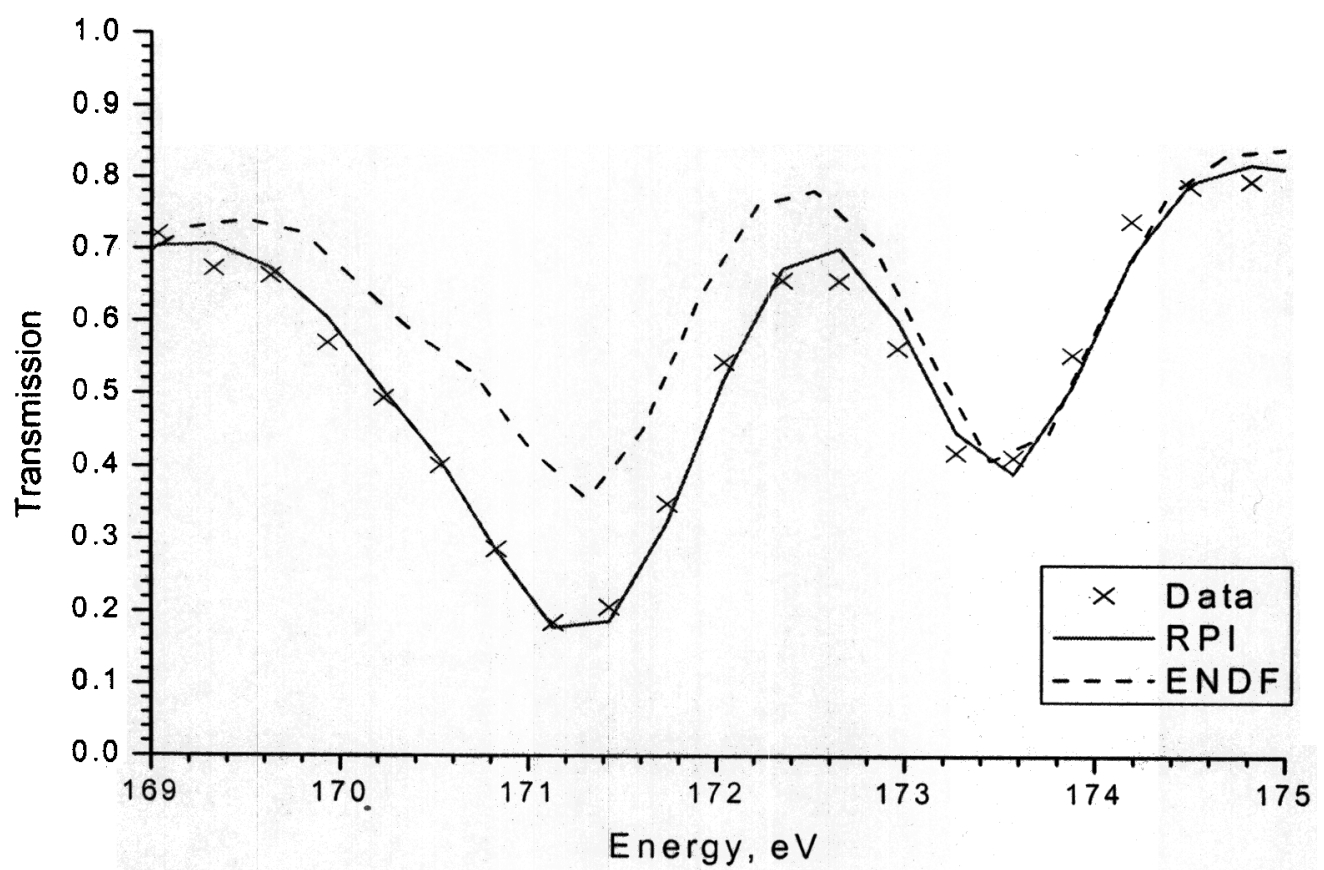

Figure 39 - 5.08-mm (200-mil) metal Gd epithermal transmission data in the $172 \mathrm{eV}$ region. The RPI fit represents a combined 15-sample SAMMY fit to epithermal metal transmission and capture data.

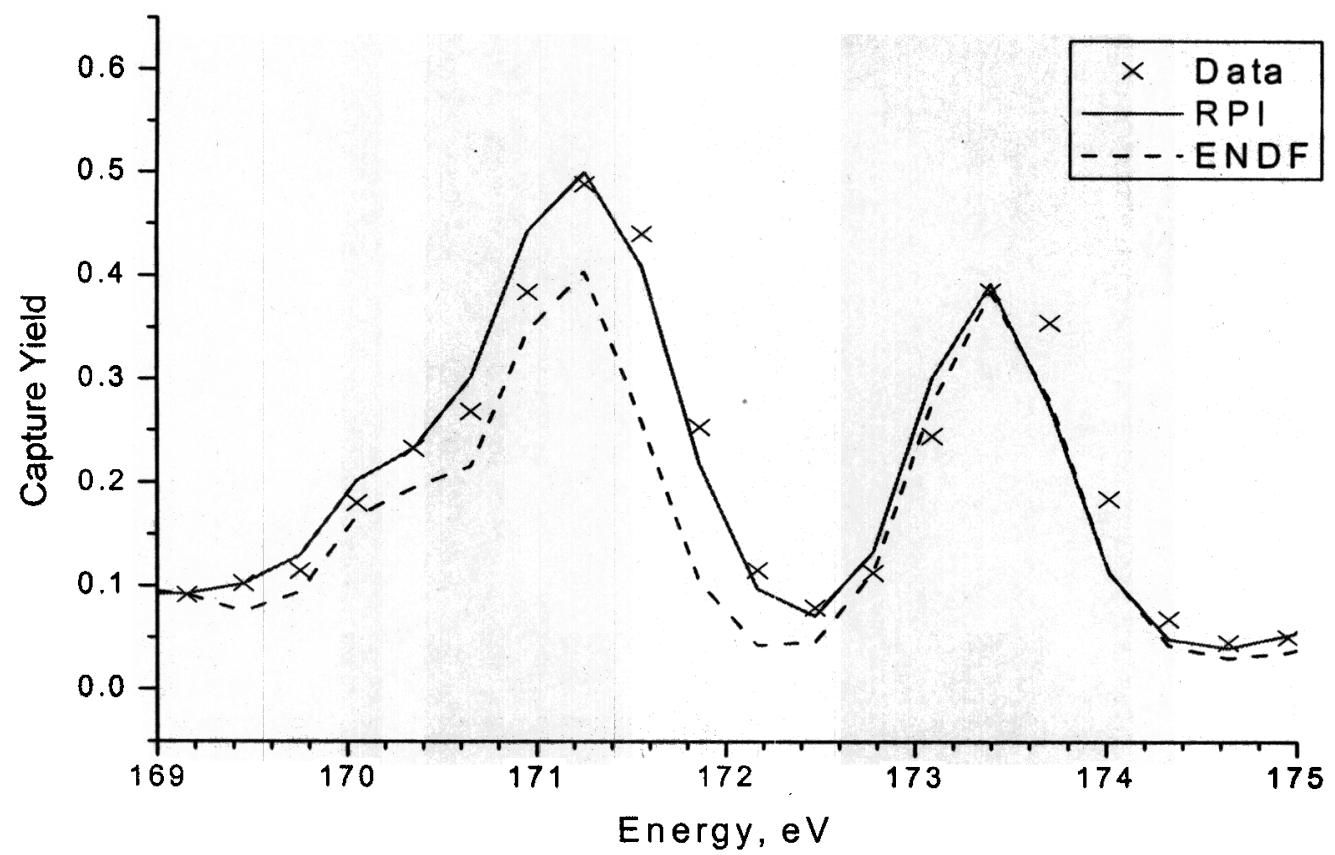

Figure 40 - 2.54-mm (100-mil) metal Gd epithermal capture data in the $172 \mathrm{eV}$ region. The RPI fit represents a combined 15-sample SAMMY fit to epithermal metal transmission and capture data. 


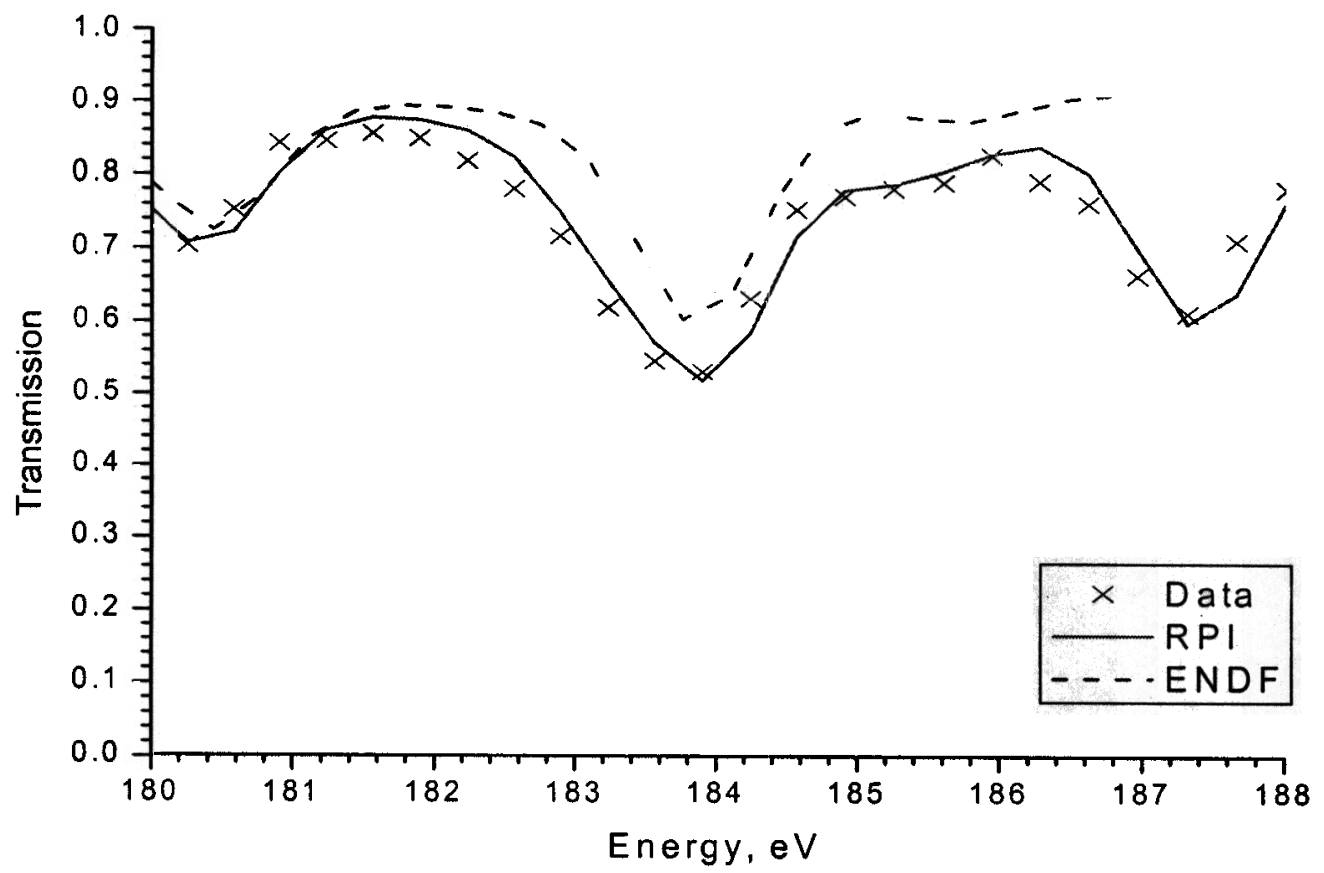

Figure 41 - 5.08-mm (200-mil) metal Gd epithermal transmission data in the $184 \mathrm{eV}$ region. The RPI fit represents a combined 15-sample SAMMY fit to epithermal metal transmission and capture data.

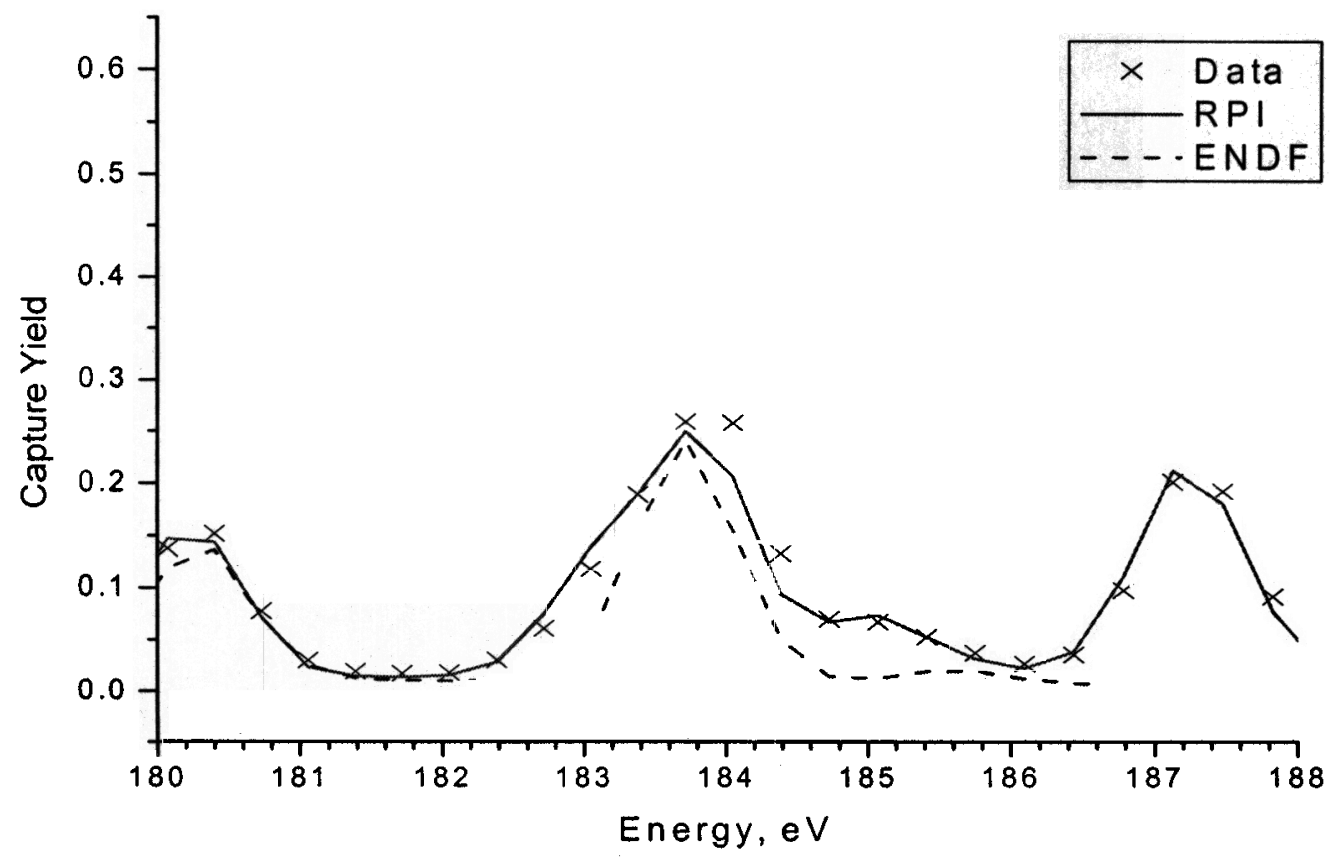

Figure 42 - 2.54-mm (100-mil) metal Gd epithermal capture data in the $184 \mathrm{eV}$ region. The RPI fit represents a combined 15-sample SAMMY fit to epithermal metal transmission and capture data. 


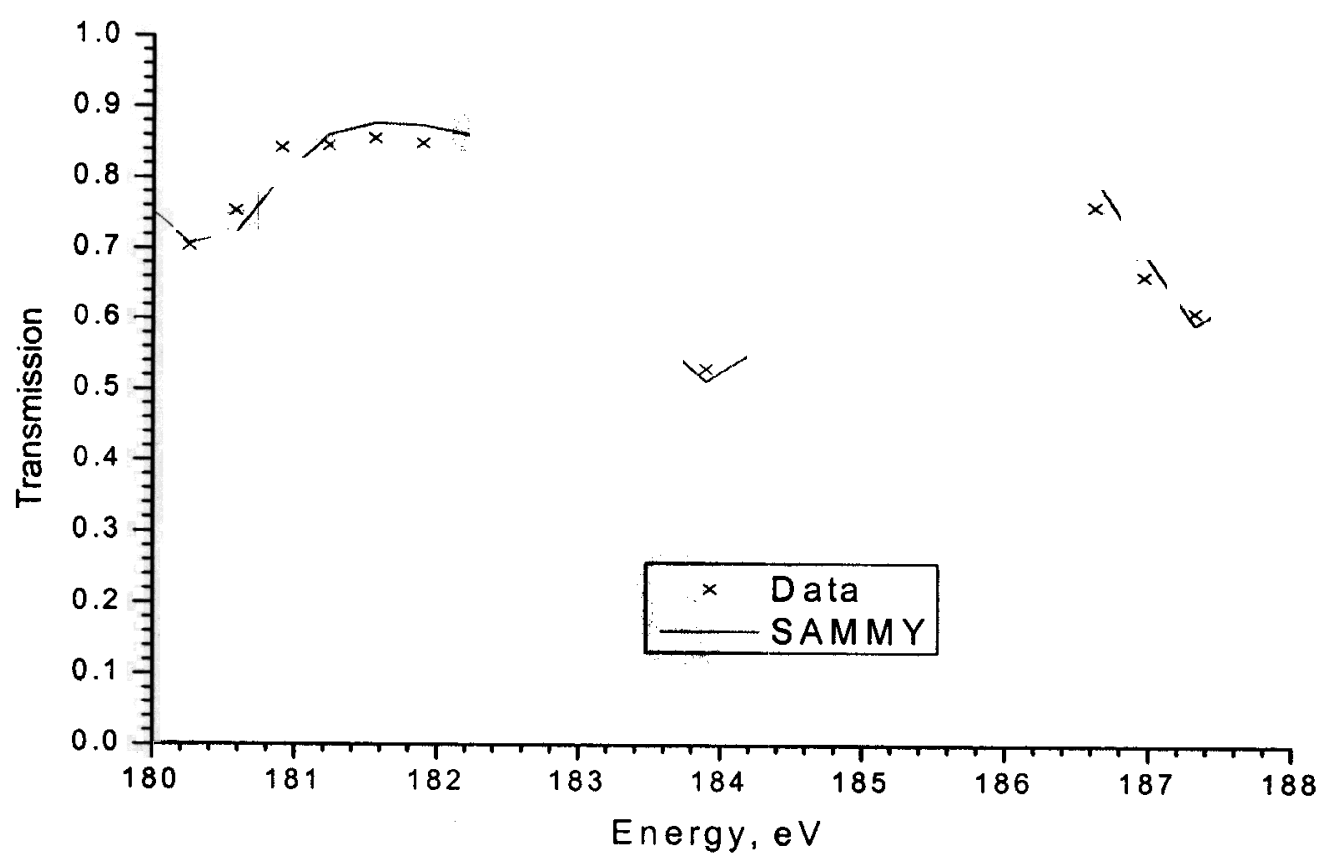

Figure 43 - 5.08-mm (200-mil) metal Gd epithermal transmission data in the $184 \mathrm{eV}$ region. The SAMMY fit has no additional resonance at $185.1 \mathrm{eV}$. Comparison to Figure 41 shows that one is needed.

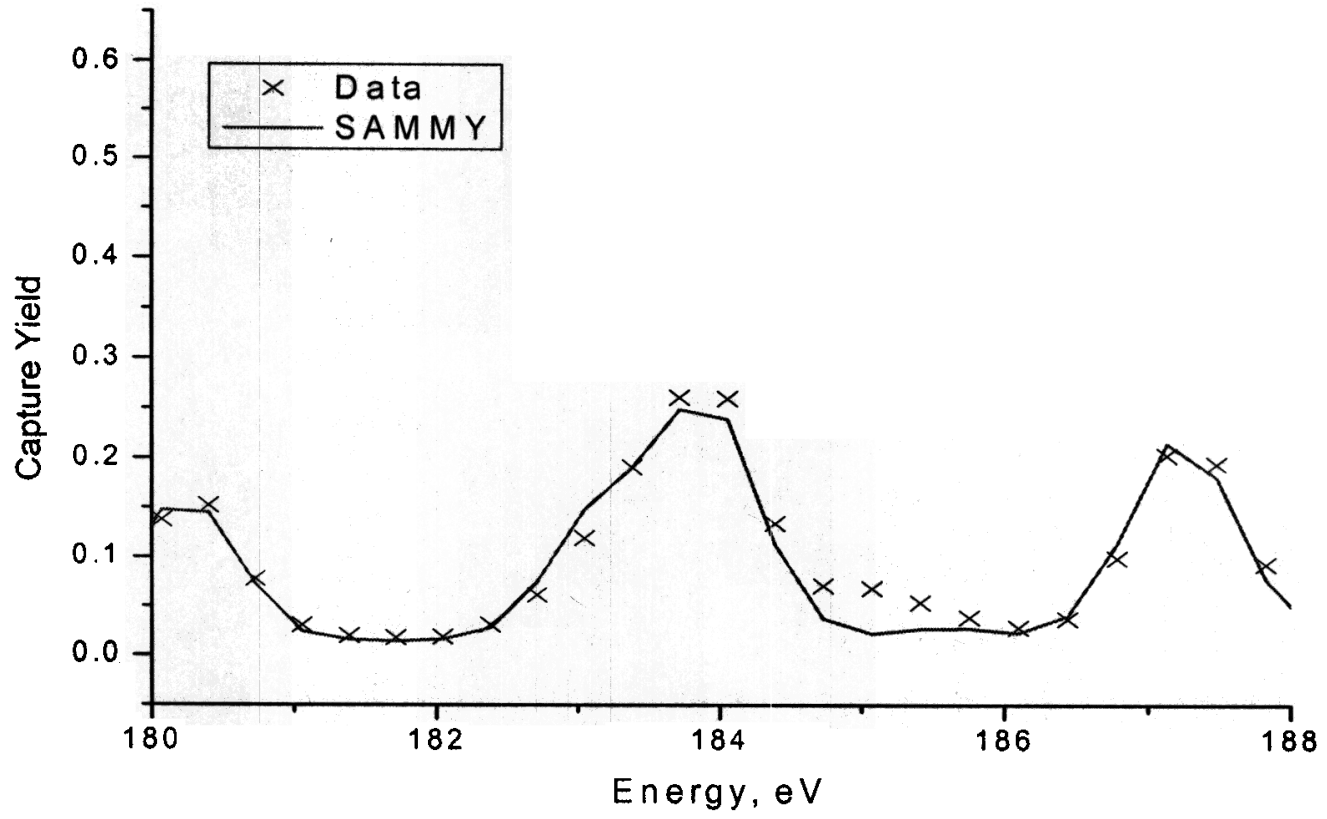

Figure $44-2.54-\mathrm{mm}$ (100-mil) metal Gd epithermal capture data in the $184 \mathrm{eV}$ region. The SAMMY fit has no additional resonance at $185.1 \mathrm{eV}$. Comparison to Figure 42 shows that one is needed. 


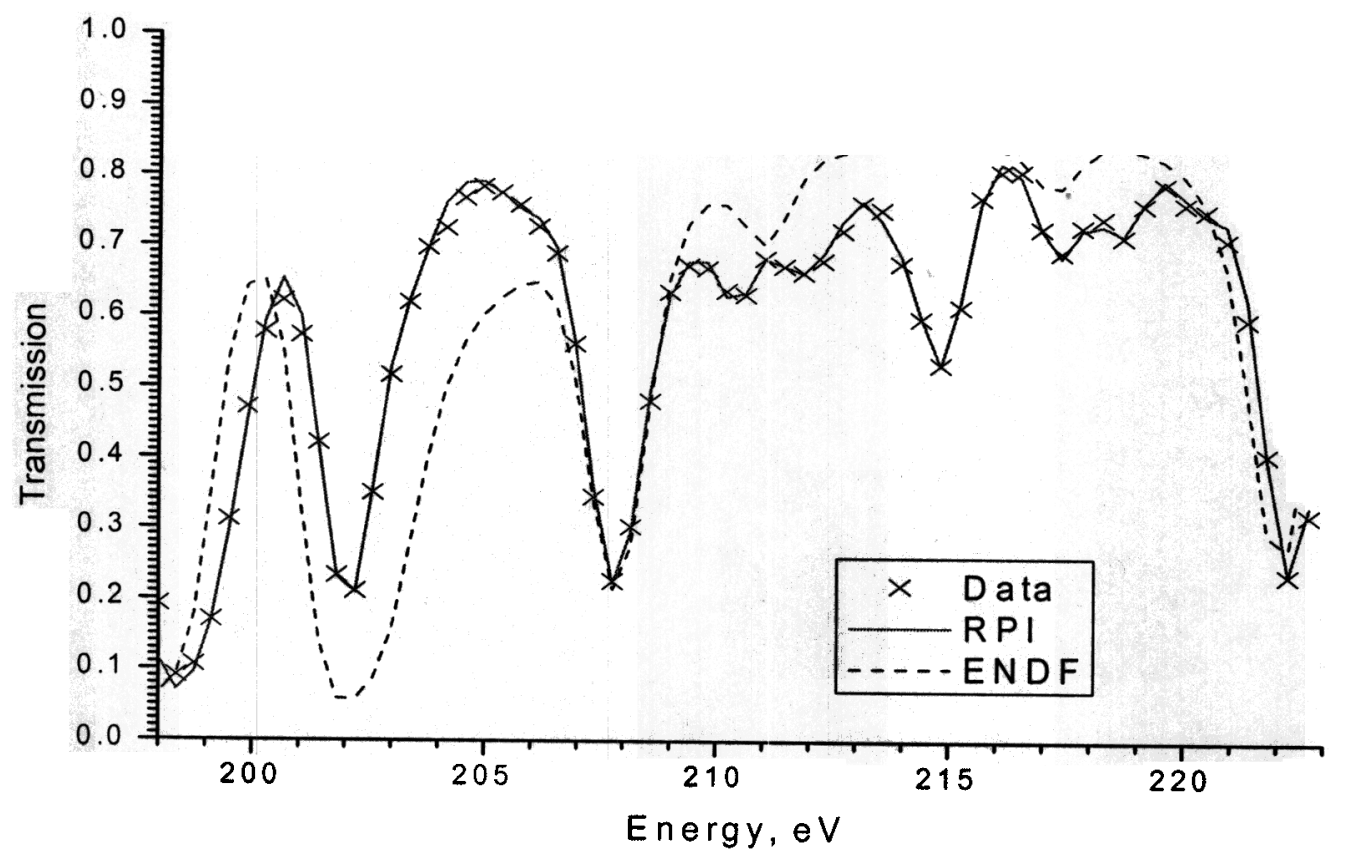

Figure 45 - 5.08-mm (200-mil) metal Gd epithermal transmission data in the $200-220 \mathrm{eV}$ region. The RPI fit represents a combined 15-sample SAMMY fit to epithermal metal transmission and capture data.

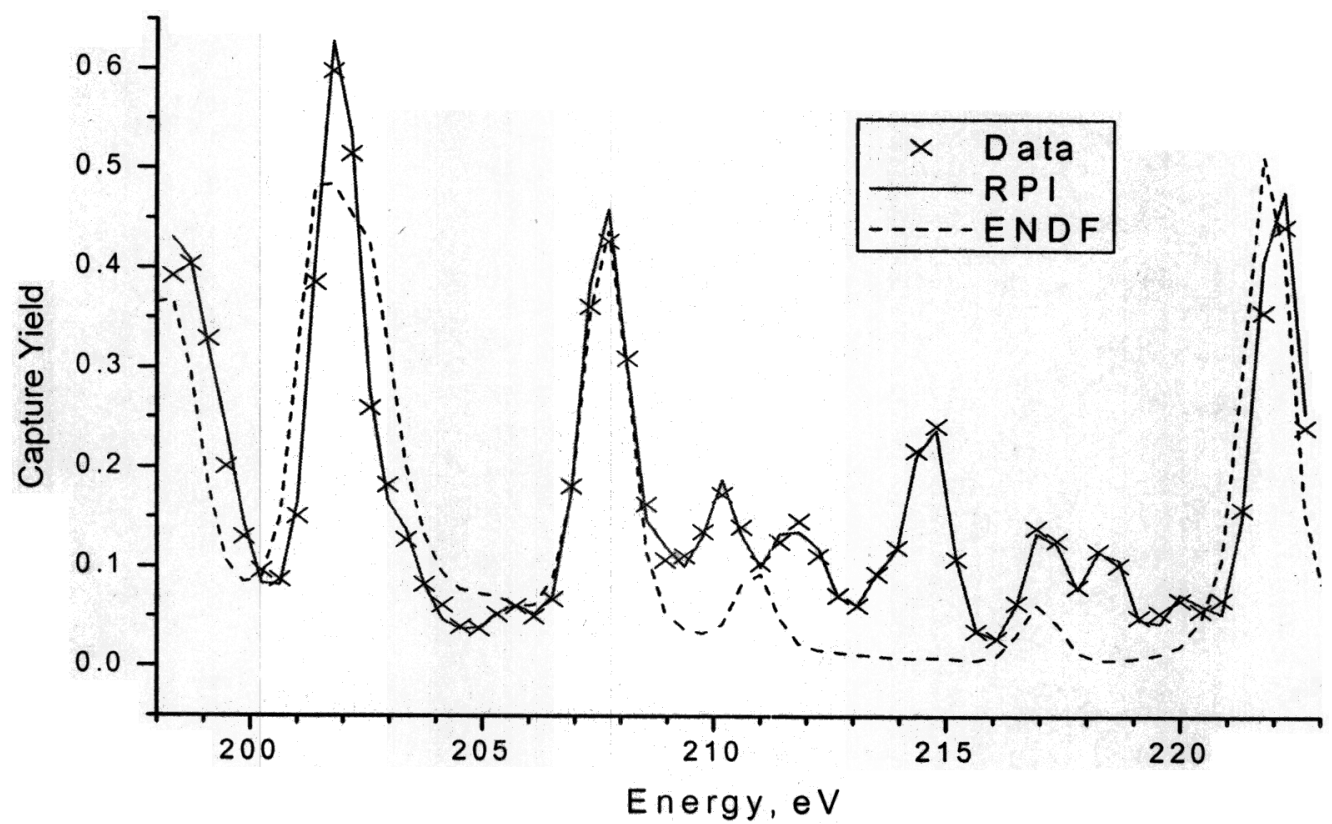

Figure 46 - 2.54-mm (100-mil) metal Gd epithermal capture data in the 200-220 eV region. The RPI fit represents a combined 15-sample SAMMY fit to epithermal metal transmission and capture data. 


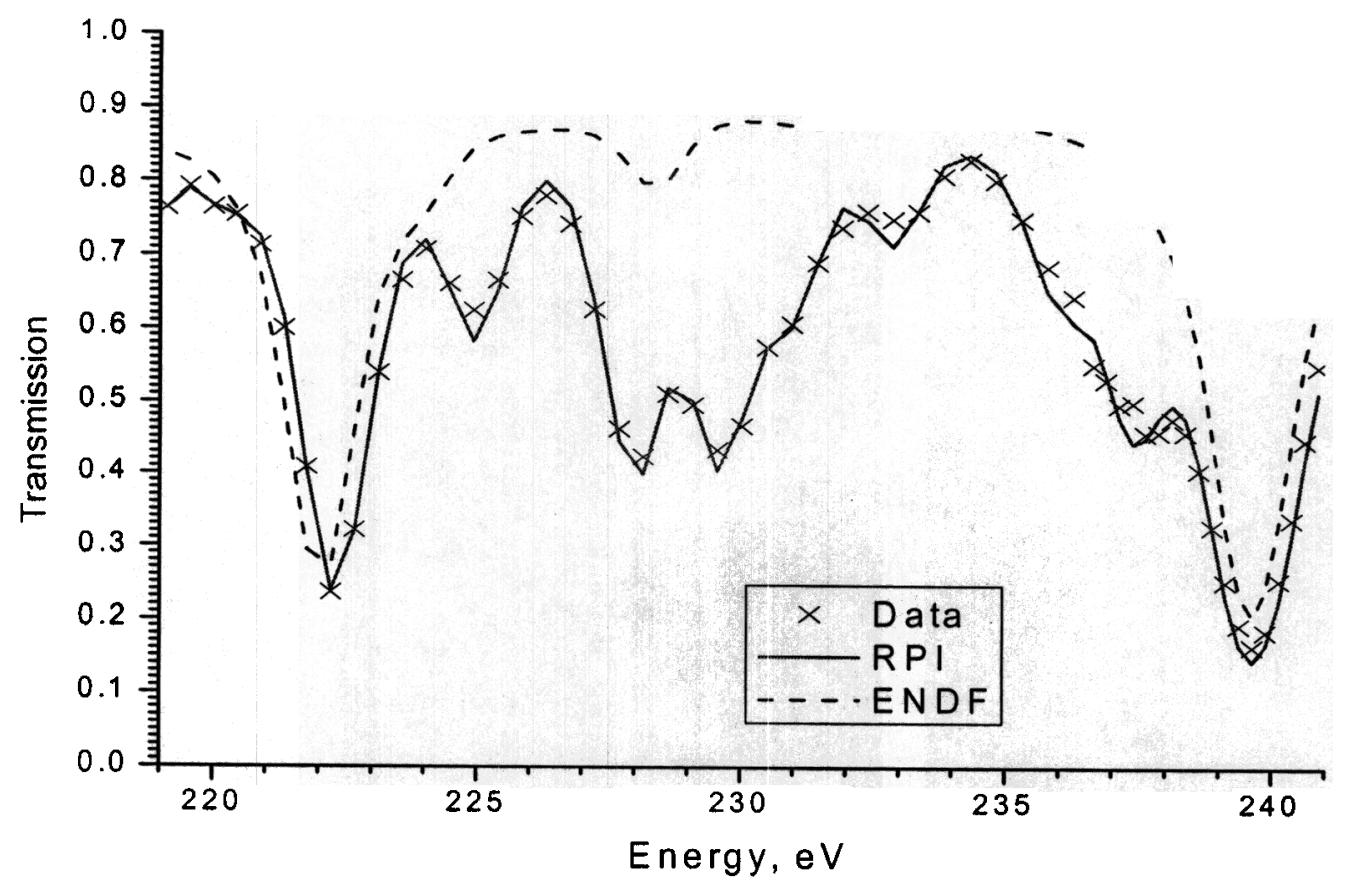

Figure 47 - 5.08-mm (200-mil) metal Gd epithermal transmission data in the $220-240 \mathrm{eV}$ region. The RPI fit represents a combined 15-sample SAMMY fit to epithermal metal transmission and capture data.

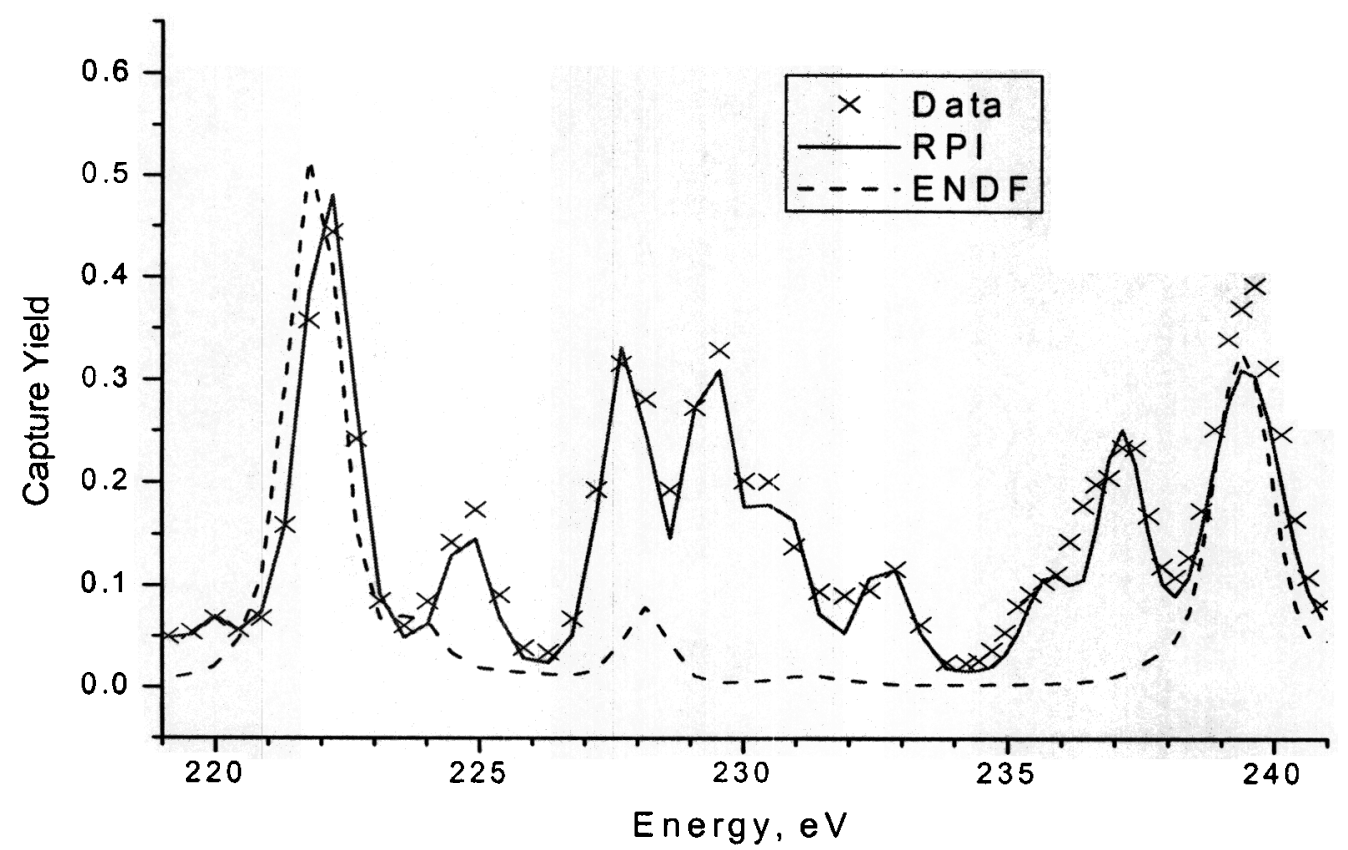

Figure 48 - 2.54-mm (100-mil) metal Gd epithermal capture data in the 220-240 eV region. The RPI fit represents a combined 15-sample SAMMY fit to epithermal metal transmission and capture data. 


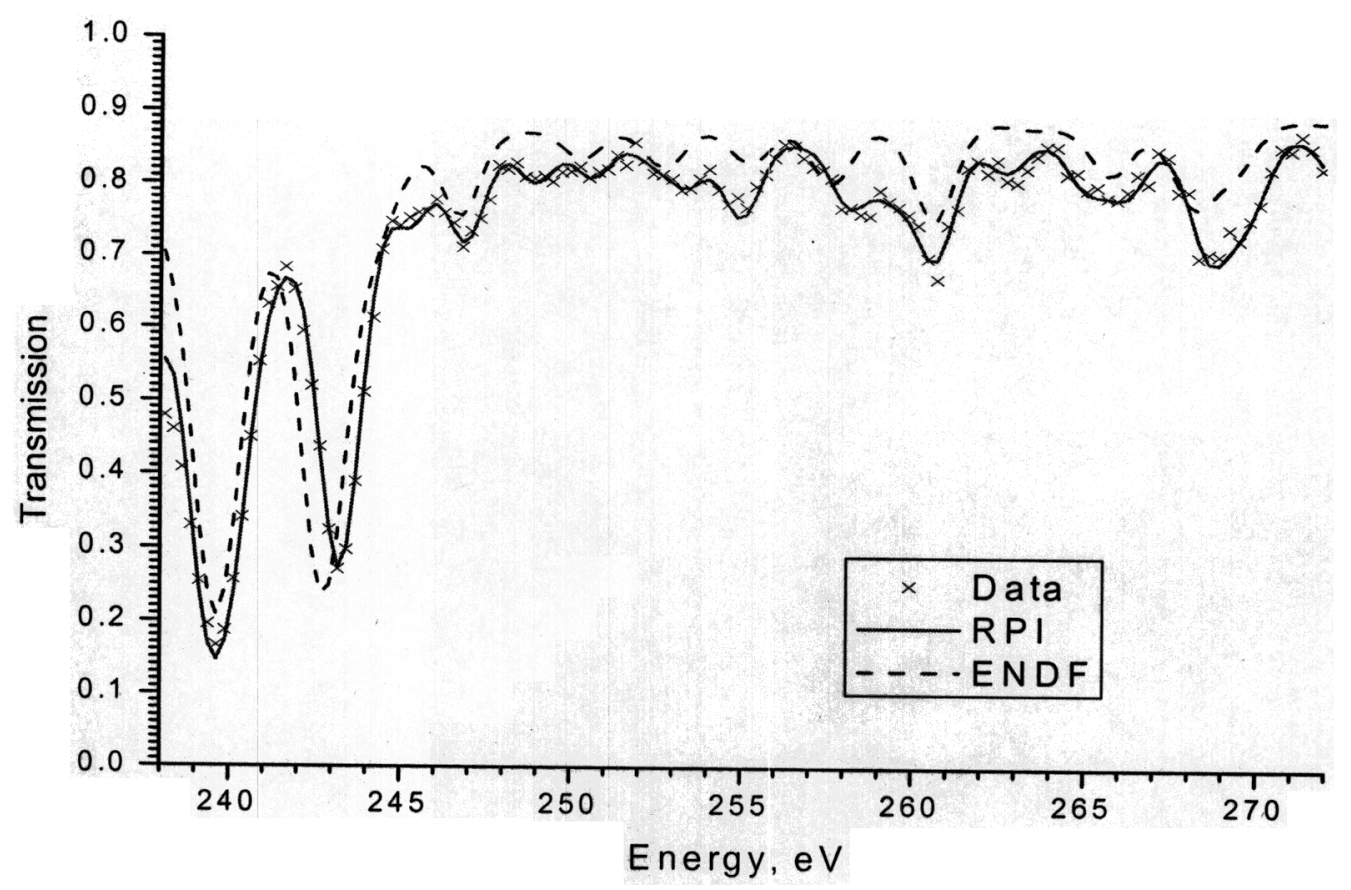

Figure 49 - 5.08-mm (200-mil) metal Gd epithermal transmission data in the 240-270 eV region. The RPI fit represents a combined 15-sample SAMMY fit to epithermal metal transmission and capture data.

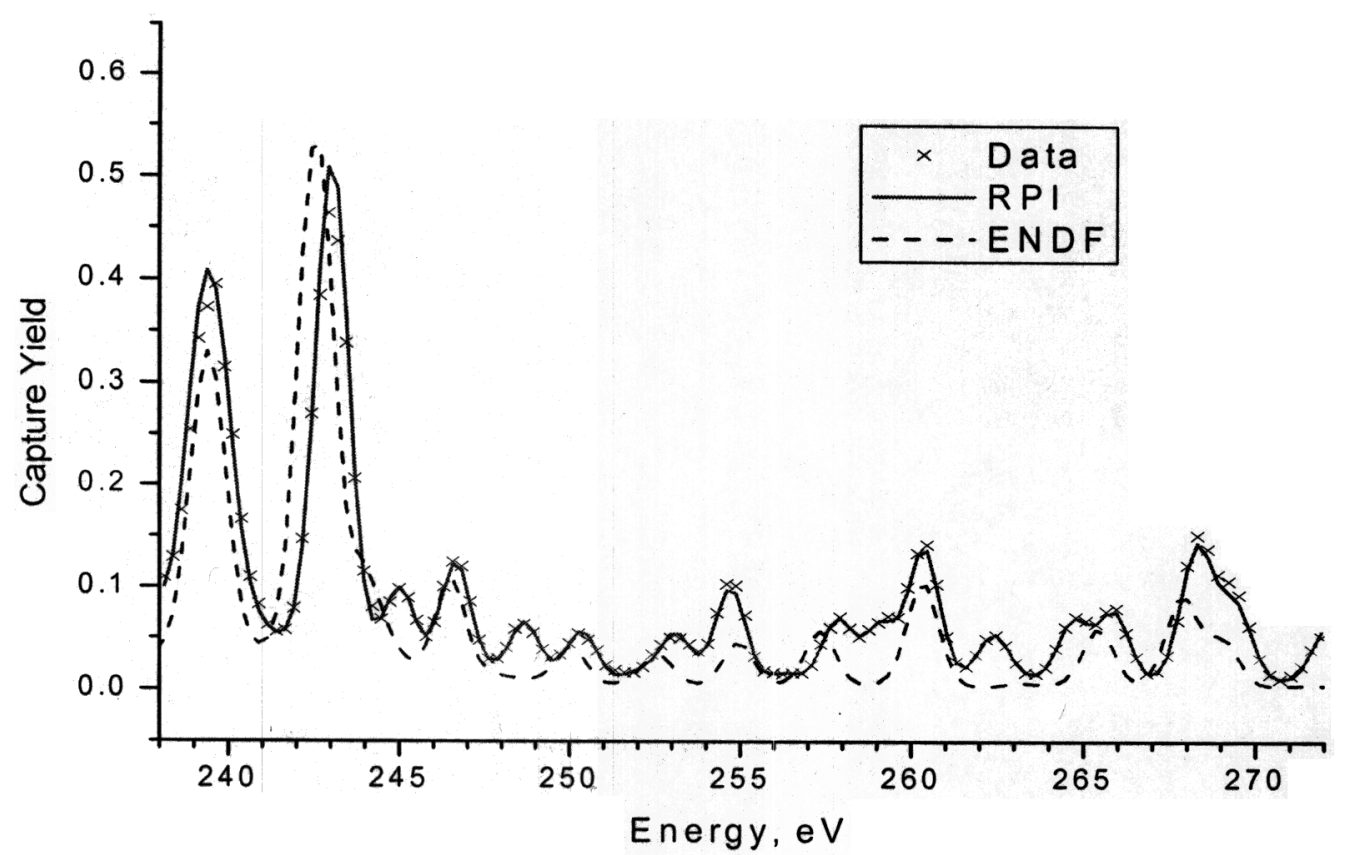

Figure 50 - 2.54-mm (100-mil) metal Gd epithermal capture data in the 240-270 eV region. The RPI fit represents a combined 15-sample SAMMY fit to epithermal metal transmission and capture data. 


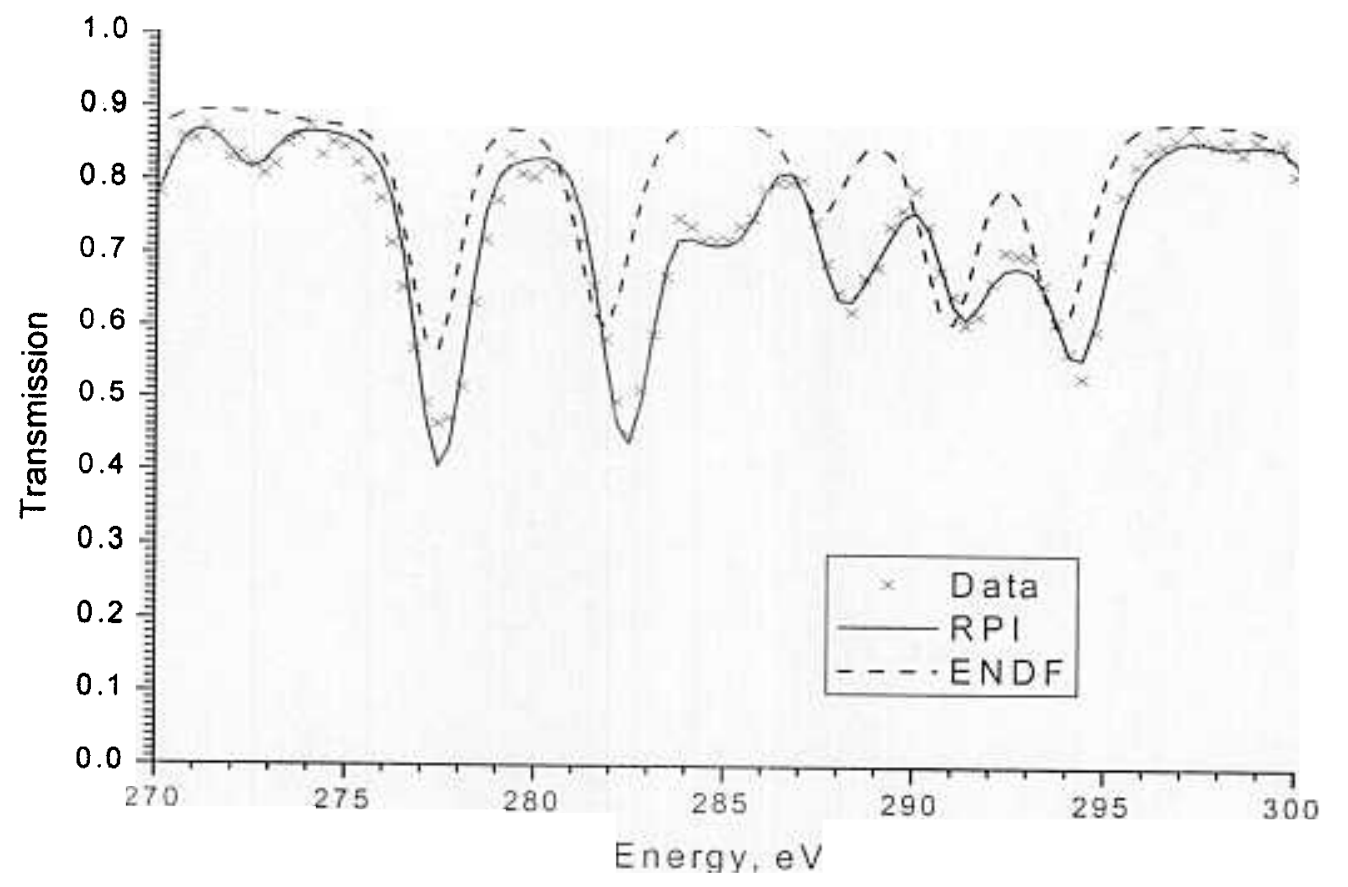

Figure $51-5.08-\mathrm{mm}(200-\mathrm{mil})$ metal Gd epithermal transmission data in the $270-300 \mathrm{eV}$ region. The RPI fit represents a combined 15-sample SAMMY fit to epithermal metal transmission and capture data.

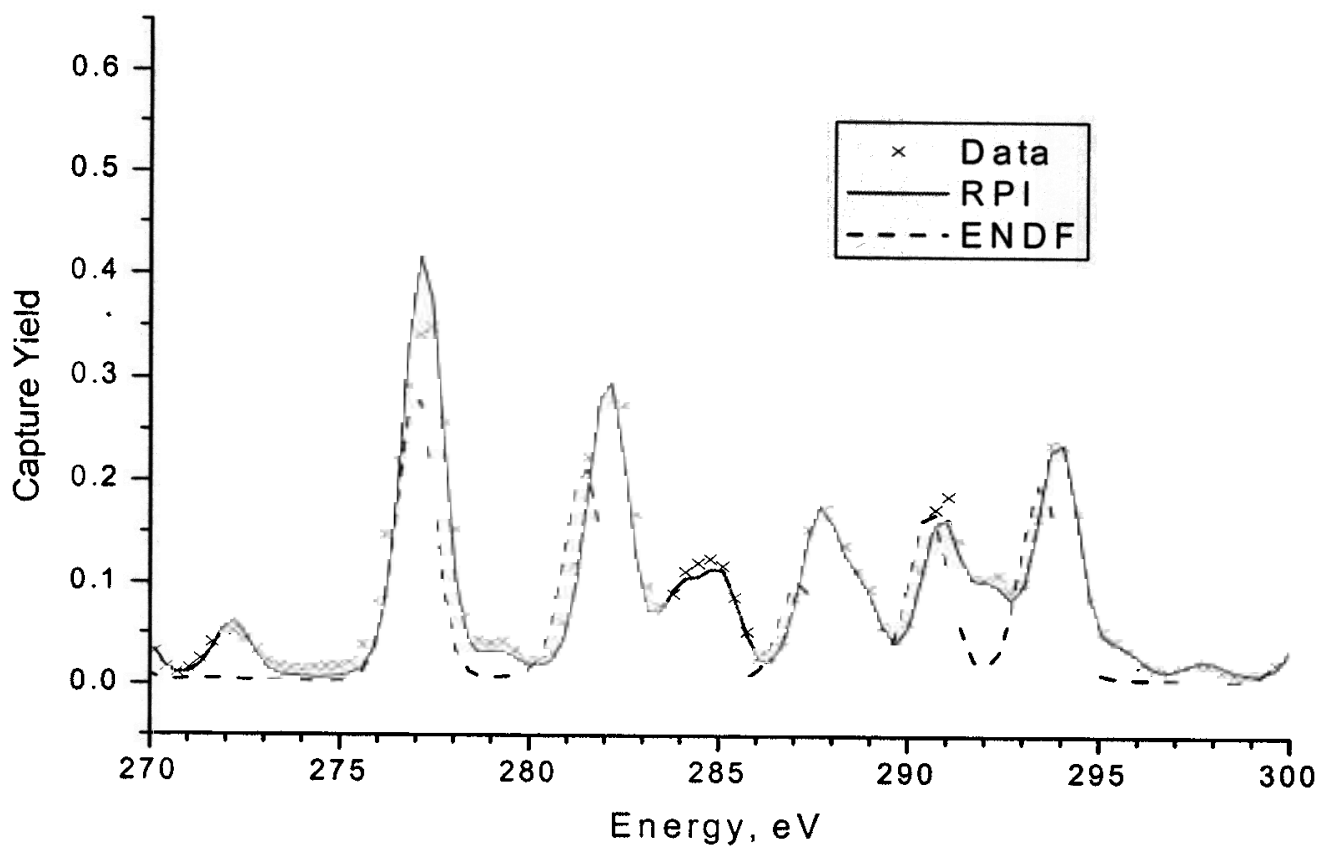

Figure 52 - 2.54-mm (100-mil) metal Gd epithermal capture data in the 270-300 eV region. The RPI fit represents a combined 15-sample SAMMY fit to epithermal metal transmission and capture data. 


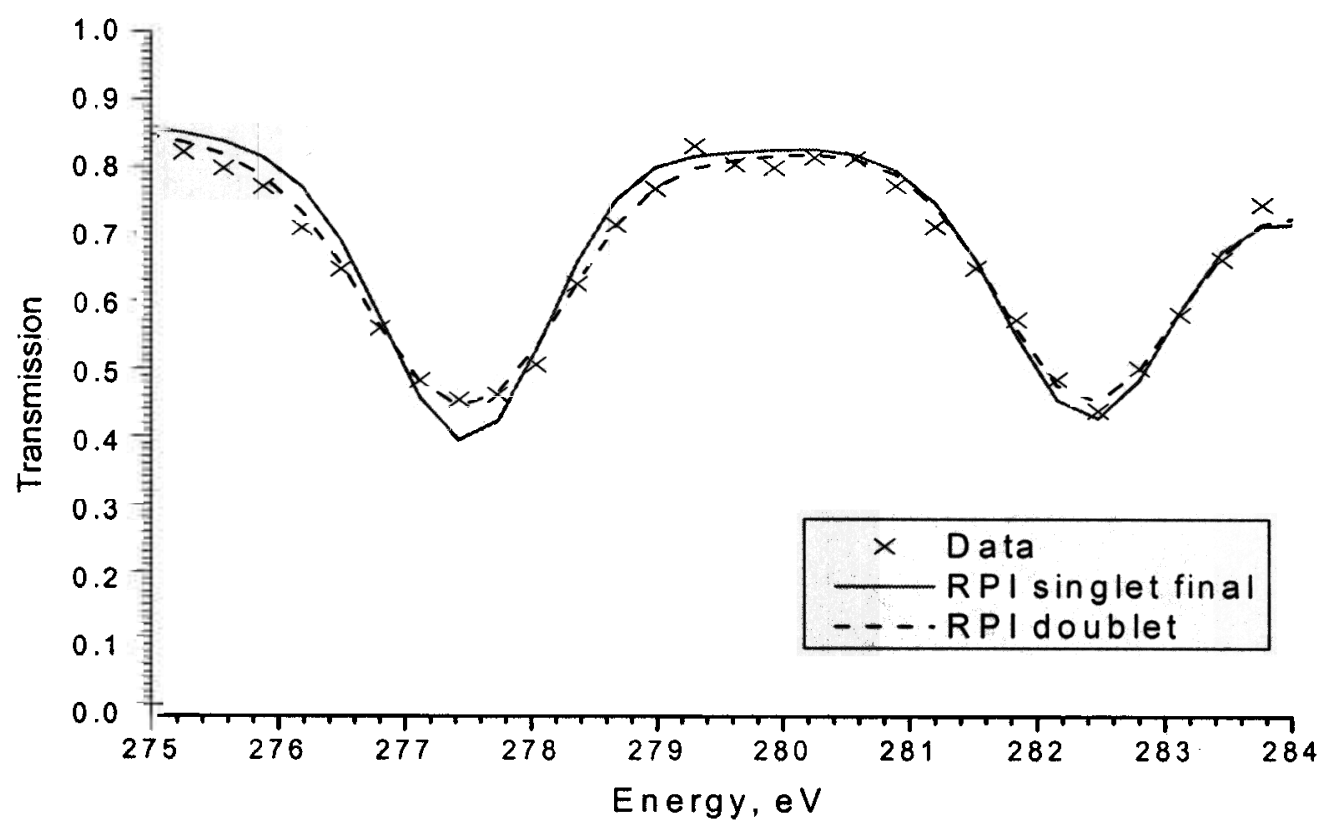

Figure 53 - 5.08-mm (200-mil) metal Gd epithermal transmission data in the $275-284 \mathrm{eV}$ region. The RPI fits represent combined 15-sample SAMMY fits to epithermal metal transmission and capture data. The "doublet" curve includes doublets at 277 and $282 \mathrm{eV}$. The "singlet" curve includes single resonances at 277 and $282 \mathrm{eV}$.

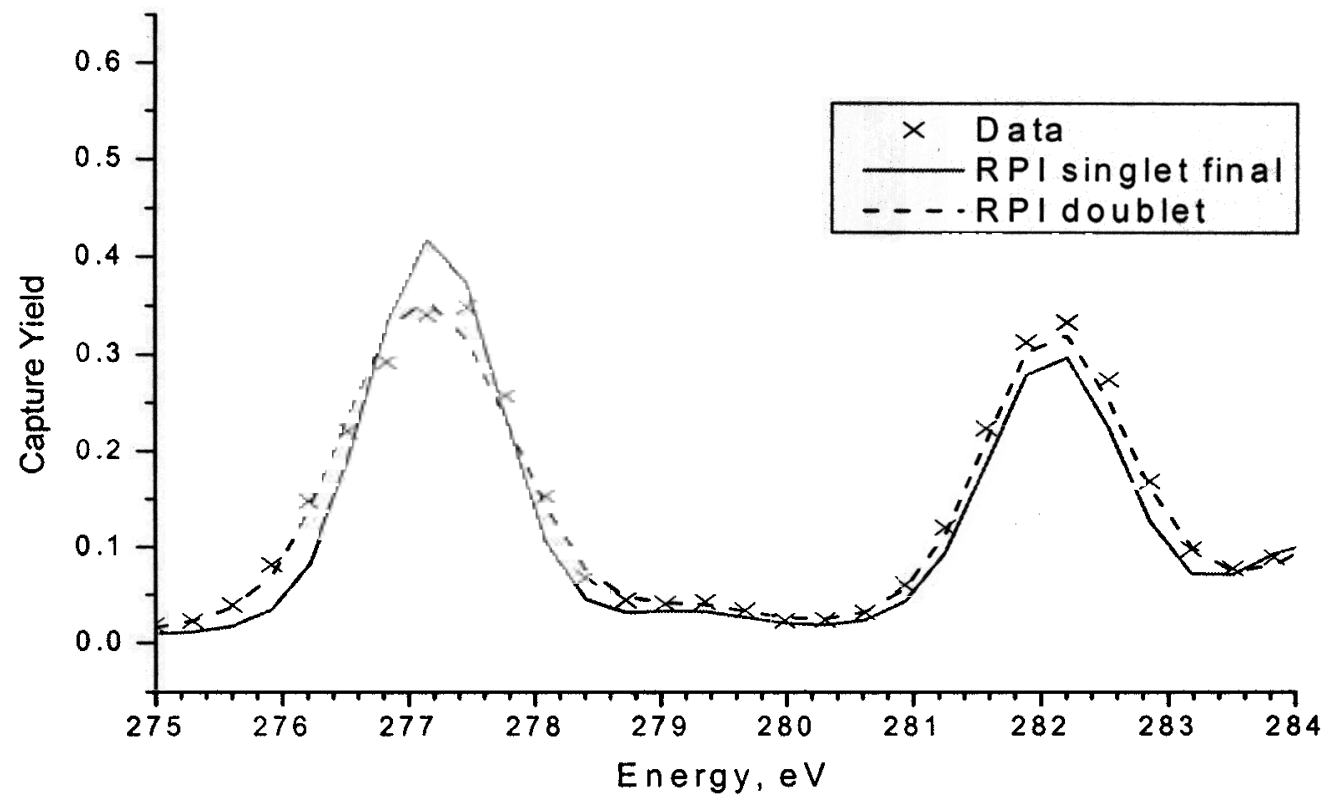

Figure 54 - 2.54-mm (100-mil) metal Gd epithermal capture data in the $275-284 \mathrm{eV}$ region. The RPI fits represent combined 15-sample SAMMY fits to epithermal metal transmission and capture data. The "doublet" curve includes doublets at 277 and $282 \mathrm{eV}$. The "singlet" curve includes single resonances at 277 and $282 \mathrm{eV}$. 


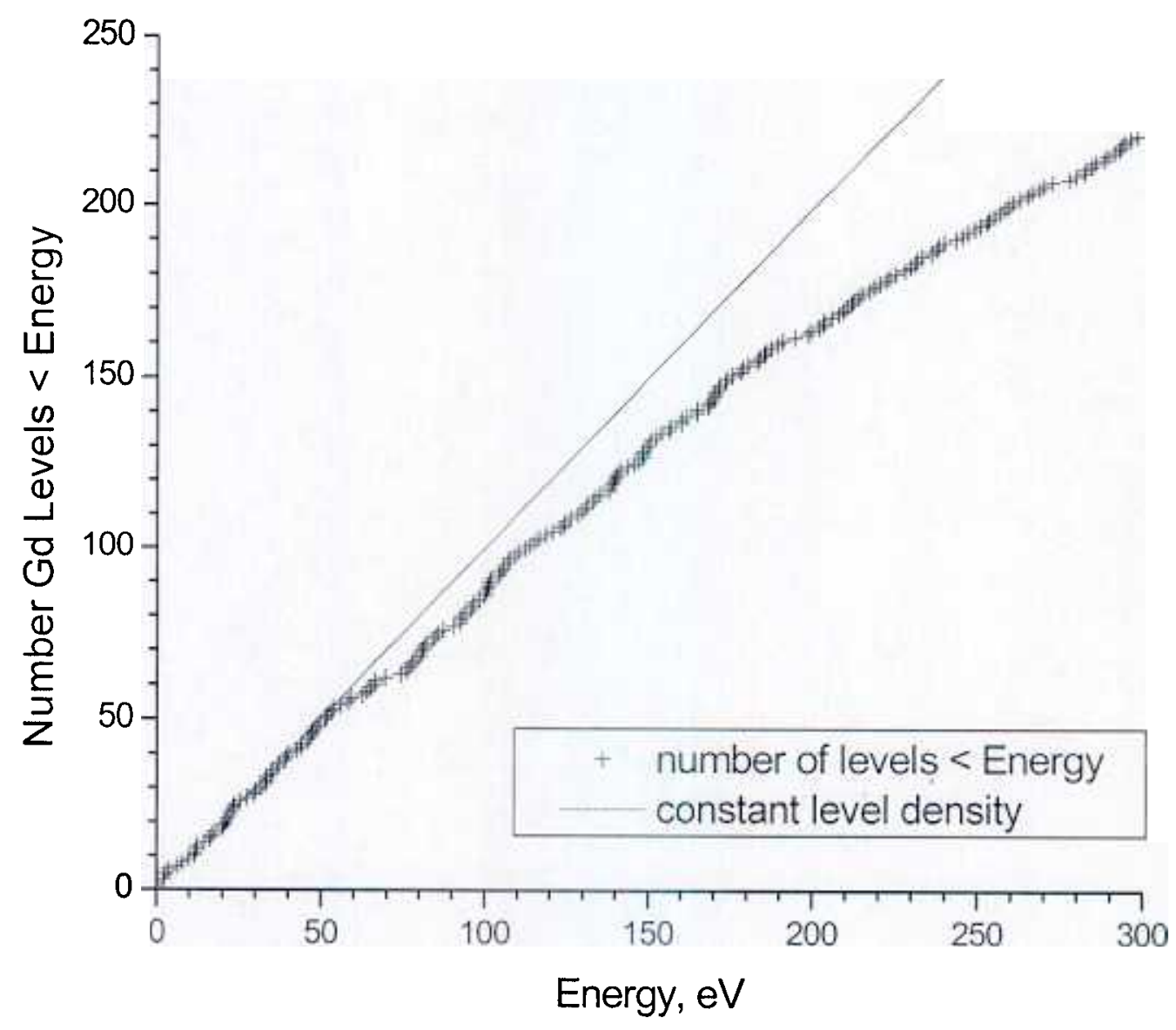

Figure 55 - 'Staircase' plot of elemental gadolinium observed levels vs energy shows a good fit to a straight line up to about $50 \mathrm{eV}$. Above $50 \mathrm{eV}$, even with the resonances added in the present analysis, the expectation of constant level density vs energy is not exceeded. 


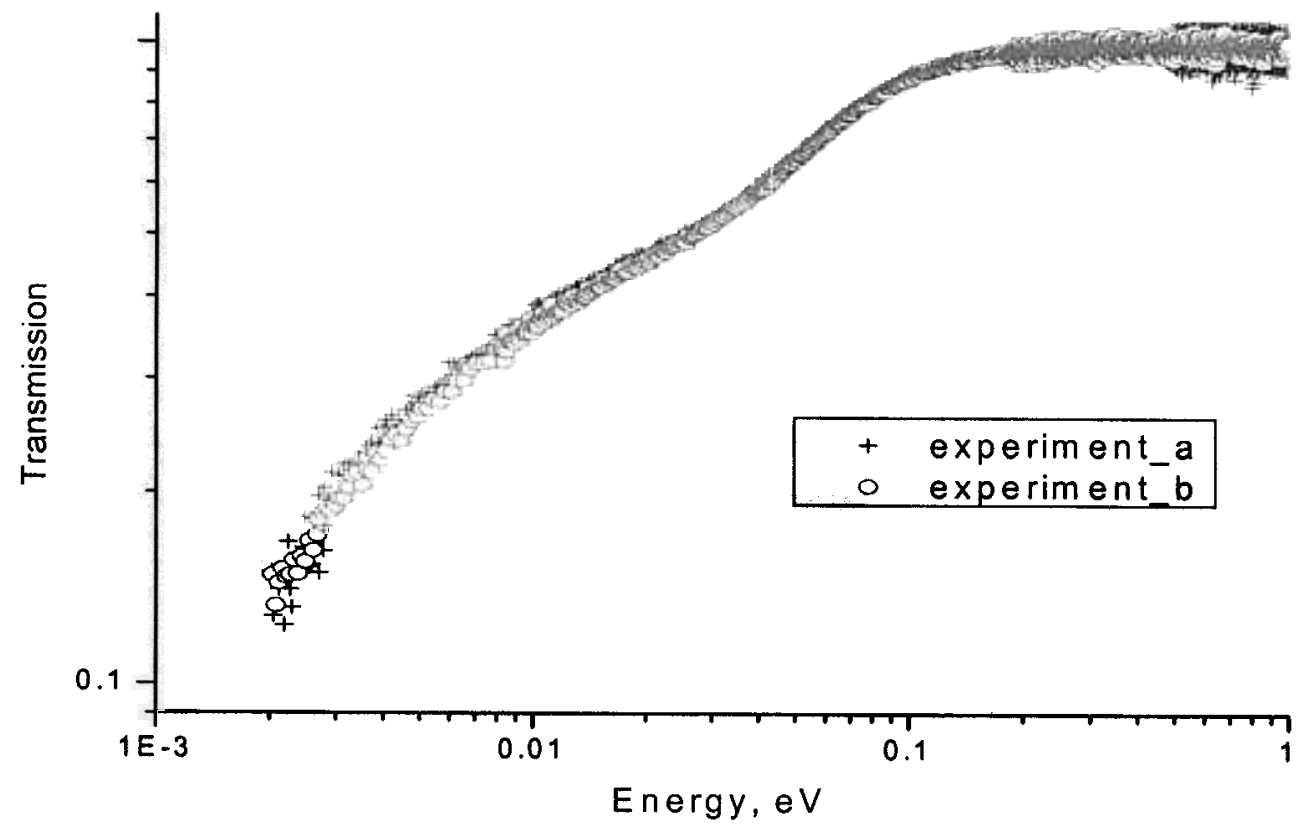

Figure 56 - Transmission data from a common sample (LX-4) in both liquid sample thermal measurements. Experimental conditions were similar. The degree of reproducibility of the measurement is an estimate of the associated systematic errors.

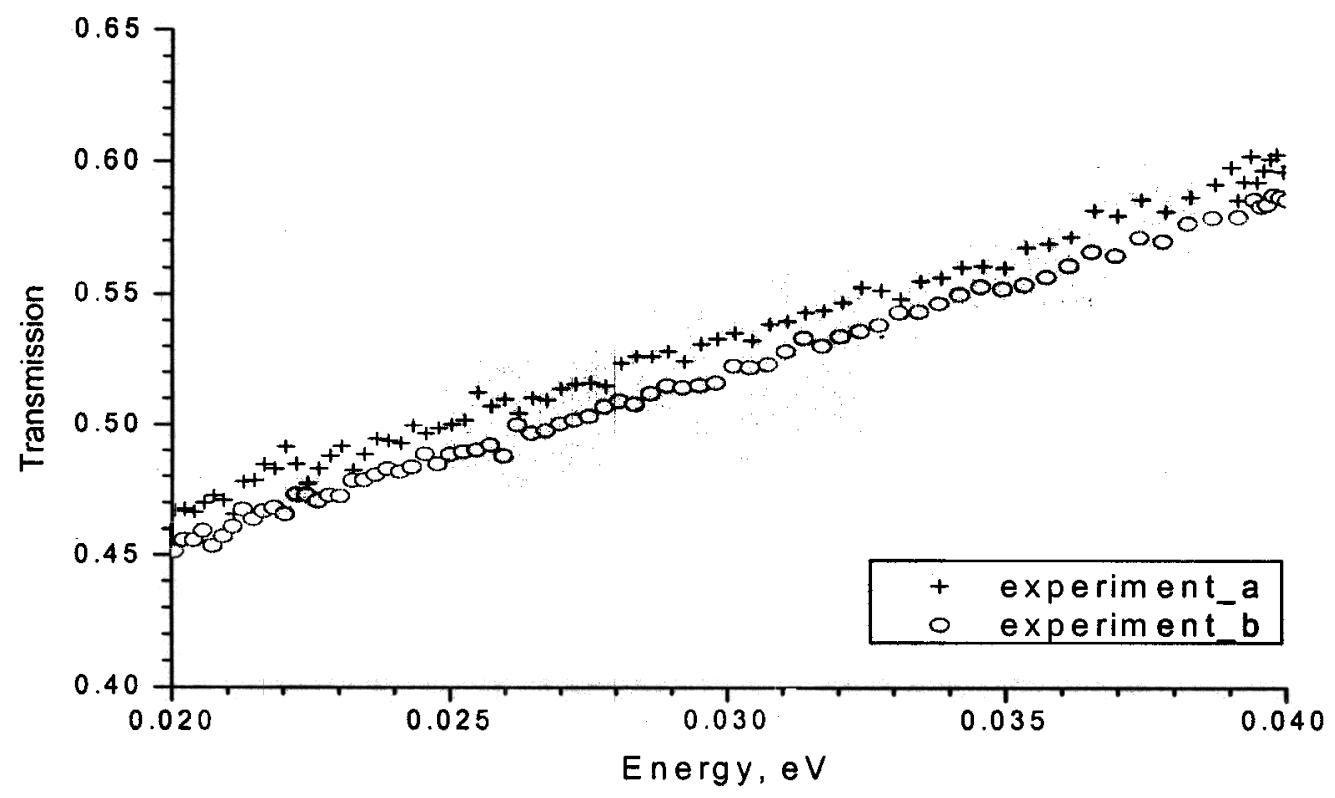

Figure 57 - Transmission data from a common sample (LX-4) in both liquid sample thermal measurements. This is a magnified view of the same data shown in Figure 56 in the region of the thermal doublet in Gd. The degree of reproducibility of the measurement is an estimate of the associated systematic errors. 


\section{Appendix A. Details of MCNP Calculations Used to Correct for Geometry/Scattering Effects}

Initial calculations using SAMMY didn't agree with experimental data. SAMMY has a limited geometry where only a single cell of a single composition can be specified. In our case a homogeneous mixture of $\mathrm{Gd}$ and $\mathrm{D}_{2} \mathrm{O}$ is contained in a quartz ring (ID=1/2", $\mathrm{OD}=2$ ", front and back plates are $1 / 16$ " thick). For this geometry to be processed in SAMMY, the quartz has to be either homogeneously "mixed" in with Gd and $\mathrm{D}_{2} \mathrm{O}$, or left out entirely. The latter approach was chosen. Background was estimated by measuring the detector response of a $\mathrm{D}_{2} \mathrm{O}$-only cell.

The experimental data were processed by subtracting $\mathrm{D}_{2} 0$-only-cell data from the $\mathrm{Gd}$ plus $\mathrm{D}_{2} 0$ cell data. Correction factors, $k$, were calculated with $\mathrm{MCNP}^{1}$ (see Figure A-1) to adjust the processed data to the SAMMY geometry.

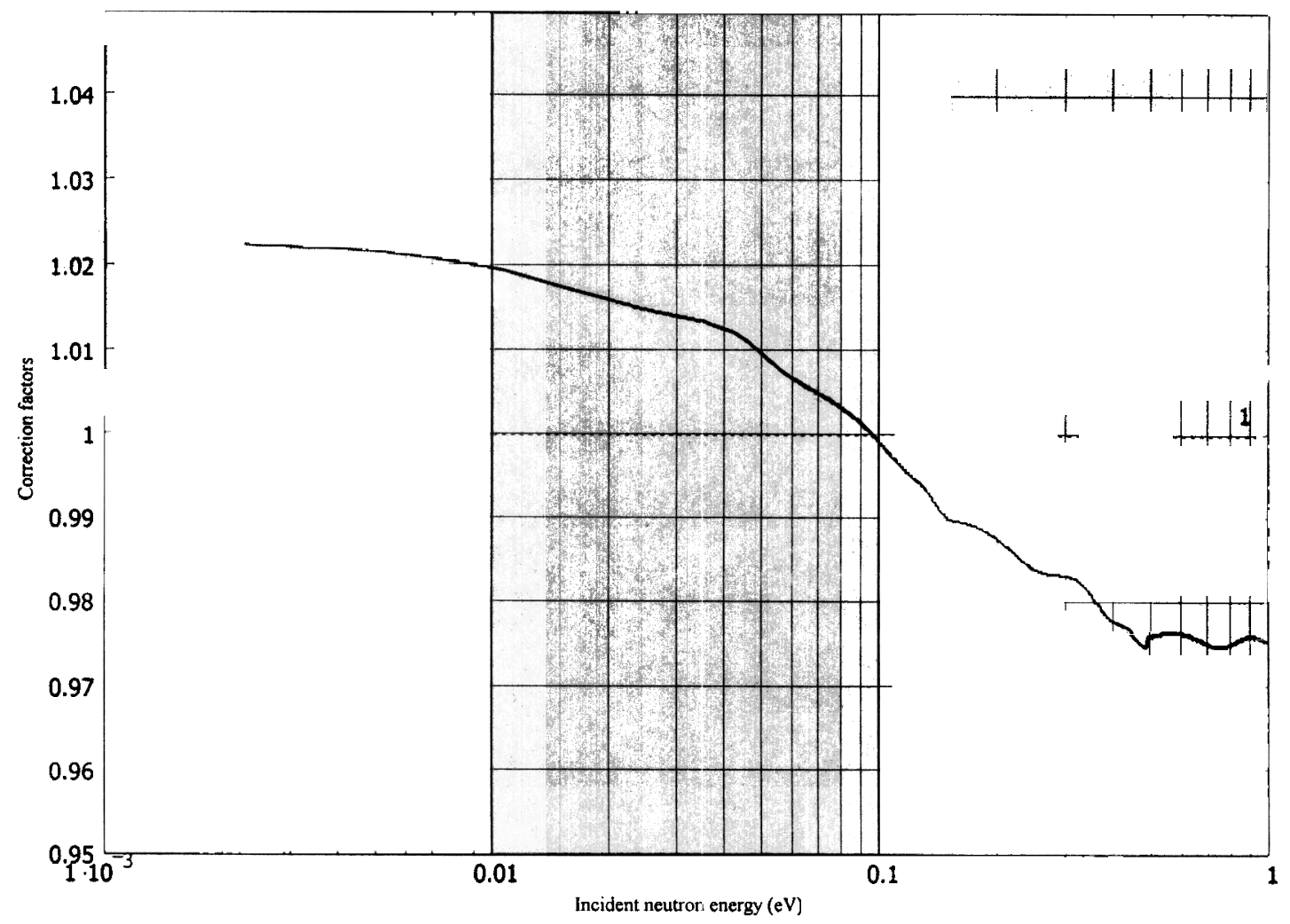

Figure A-1: Correction factors for sample LX-4.

\footnotetext{
${ }^{1}$ MCNP5 ${ }^{\mathrm{TM}}$ - A General Monte Carlo N-Particle Transport Code, Version 5. The Regents of the University of California, Los Alamos National Laboratory
} 
These factors account for the fact that neutrons can be scattered by quartz and subsequently captured in Gd. These factors were calculated using three MCNP models:

1. A full representation of the cell: a homogeneous mix of $\mathrm{Gd}$ and $\mathrm{D}_{2} 0$ enclosed in quartz housing (see Figure A-3: MCNP deck (Full cell).)

2. The $\mathrm{Gd}$ and $\mathrm{D}_{2} \mathrm{O}$ mix, retaining its geometry, but not surrounded by quartz (see Figure A-4: MCNP deck (No quartz).)

3. The original cell filled with $\mathrm{D}_{2} \mathrm{O}$ only (see Figure A-5: MCNP deck (No Gd).)

Model \#1 is the experimental setup as measured. Model \#3 represents the background due to quartz and $\mathrm{D}_{2} \mathrm{O}$. Model $\# 2$ is the SAMMY geometry.

The resultant data were processed with Mathcad ${ }^{2}$ to generate the following plot of capture yields as a function of energy (Figure A-2).

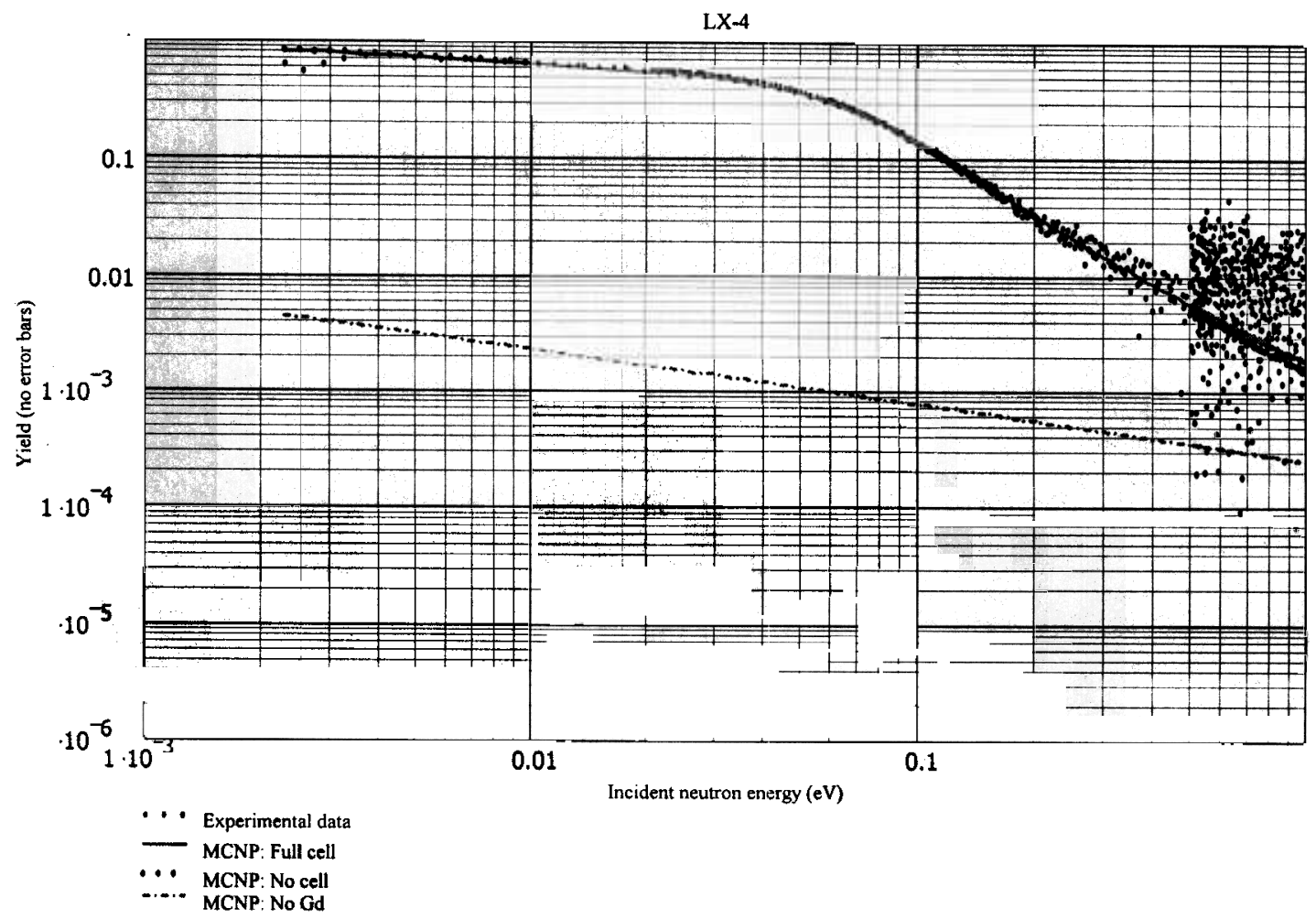

Figure A-2: Capture yields in different media.

Note that as the energy goes up (Figure A-2) the capture yield in the quartz cell with $\mathrm{D}_{2} \mathrm{O}$ only becomes more significant.

The correction factors $k$, equation (A-1) are ratios of the capture rates in the mix without any quartz container (Model \#2) to the difference between a full model of the cell and the quartz cell filled with heavy water only (Model \#1 - Model \#3).

${ }^{2}$ Mathcad®11 - Mathsoft ${ }^{\mathrm{TM}}$, Cambridge, MA 


$$
k=\frac{\left(G d+D_{2} O\right)_{N o-\text { quartz }}}{\left(G d+D_{2} O\right)_{Q u a r t z}-\left(D_{2} O\right)_{Q u a r t z}}
$$

As is seen from Figure A-1 the values of $k$ are on the order of $2 \%$ in the region of interest. They are above unity for neutron energies below $0.1 \mathrm{eV}$, and go below unity in the region $0.1 \mathrm{eV}<\mathrm{E}_{\mathrm{n}}<1 \mathrm{eV}$. data.

These calculated correction factors were subsequently applied to the experimental 


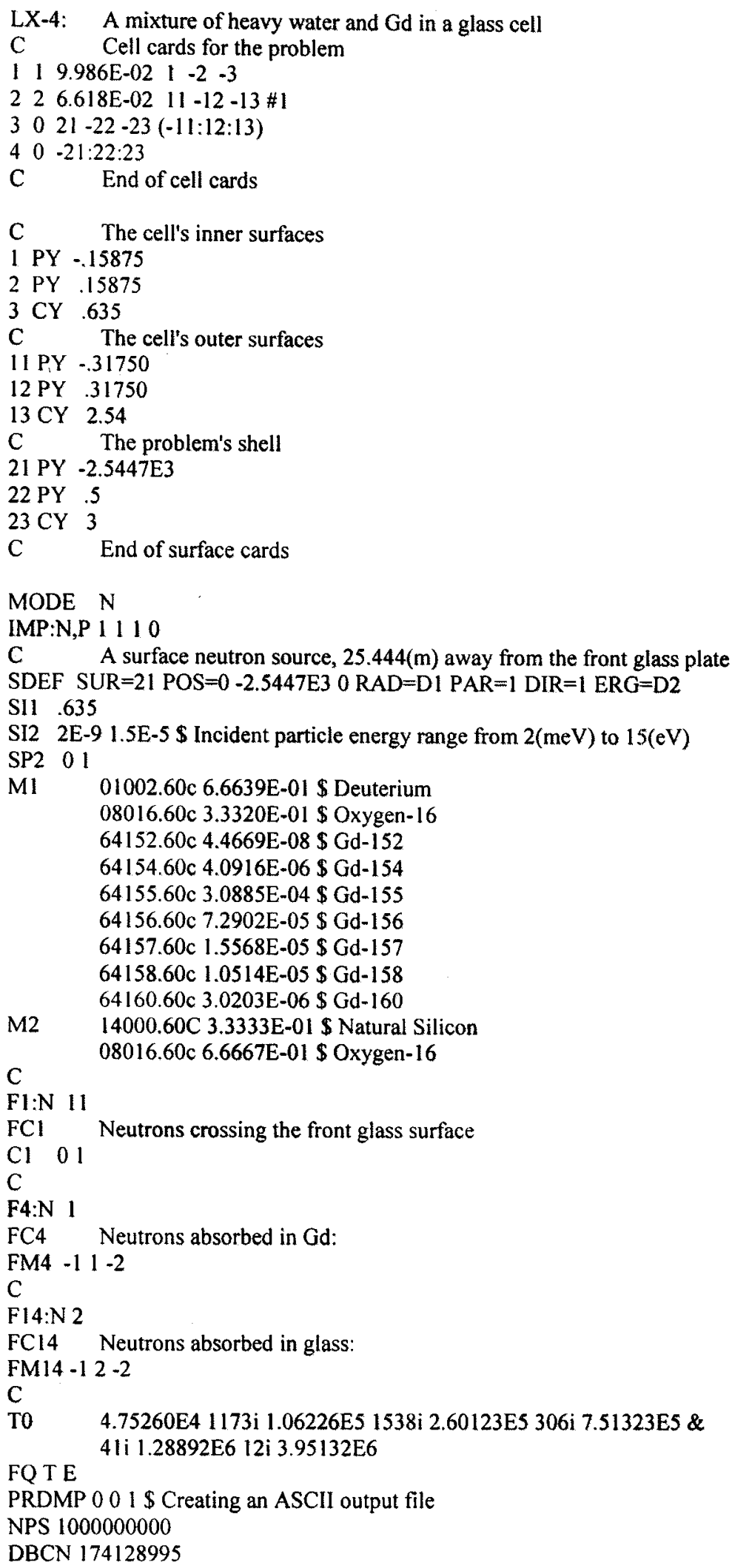

Figure A-3: MCNP deck (Full cell). 
LX-4: A mixture of heavy water and Gd, no quartz

C Cell cards for the problem

$\begin{array}{llllll}1 & 1 & 9.986 \mathrm{E}-02 & 1 & -2 & -3\end{array}$

$20021-22-23 \quad \# 1$

$\begin{array}{llll}3 & 0 & -21: 22: 23\end{array}$

C End of cell cards

C The cell's surfaces

1 PY -.15875

2 PY .15875

$3 \mathrm{CY} .635$

C The problem's shell

$21 \mathrm{PY}-2.5447 \mathrm{E} 3$

22 PY .5

$23 \mathrm{CY} 3$

C End of surface cards

MODE N

IMP:N,P 110

$C \quad$ A surface neutron source, 25.446(m) away from the front plate of the cell $\mathrm{SDEF} S \mathrm{SUR}=21$ POS=0 -2.5447E3 0 RAD=D1 $\mathrm{PAR}=1 \mathrm{DIR}=1 \mathrm{ERG}=\mathrm{D} 2$

SII .635

SI2 2E-9 1.5E-5 \$ Incident particle energy range from $2(\mathrm{meV})$ to $15(\mathrm{eV})$

$S P 201$

M1 01002.60c 6.6639E-01 \$ Deuterium $08016.60 \mathrm{c} 3.3320 \mathrm{E}-01$ \$ Oxygen-16 $64152.60 \mathrm{c} 4.4669 \mathrm{E}-08$ \$ Gd-152 $64154.60 \mathrm{c} 4.0916 \mathrm{E}-06 \$ \mathrm{Gd}-154$ $64155.60 \mathrm{c} 3.0885 \mathrm{E}-04 \$ \mathrm{Gd}-155$ $64156.60 \mathrm{c} 7.2902 \mathrm{E}-05$ \$ Gd-156 $64157.60 \mathrm{c} 1.5568 \mathrm{E}-05 \$ \mathrm{Gd}-157$ $64158.60 \mathrm{c} 1.0514 \mathrm{E}-05 \$ \mathrm{Gd}-158$

C $64160.60 \mathrm{c} 3.0203 \mathrm{E}-06 \$ \mathrm{Gd}-160$

F $1: N 1$

FCl Neutrons crossing the front surface

C. 01

$\mathrm{C}$

$\mathrm{F} 4: \mathrm{N}$

FC4 Neutrons absorbed in Gd and Heavy Water:

FM4 - $1:-2$

C

T0 4.75260E4 1173i 1.06226E5 1538i 2.60123E5 306i 7.51323E5 \&

FQ T E $41 \mathrm{i} 1.28892 \mathrm{E} 612 \mathrm{i} 3.95132 \mathrm{E} 6$

PRDMP 001 \$ Creating an ASCII output file NPS 1000000000

DBCN 350308103

Figure A-4: MCNP deck (No quartz). 


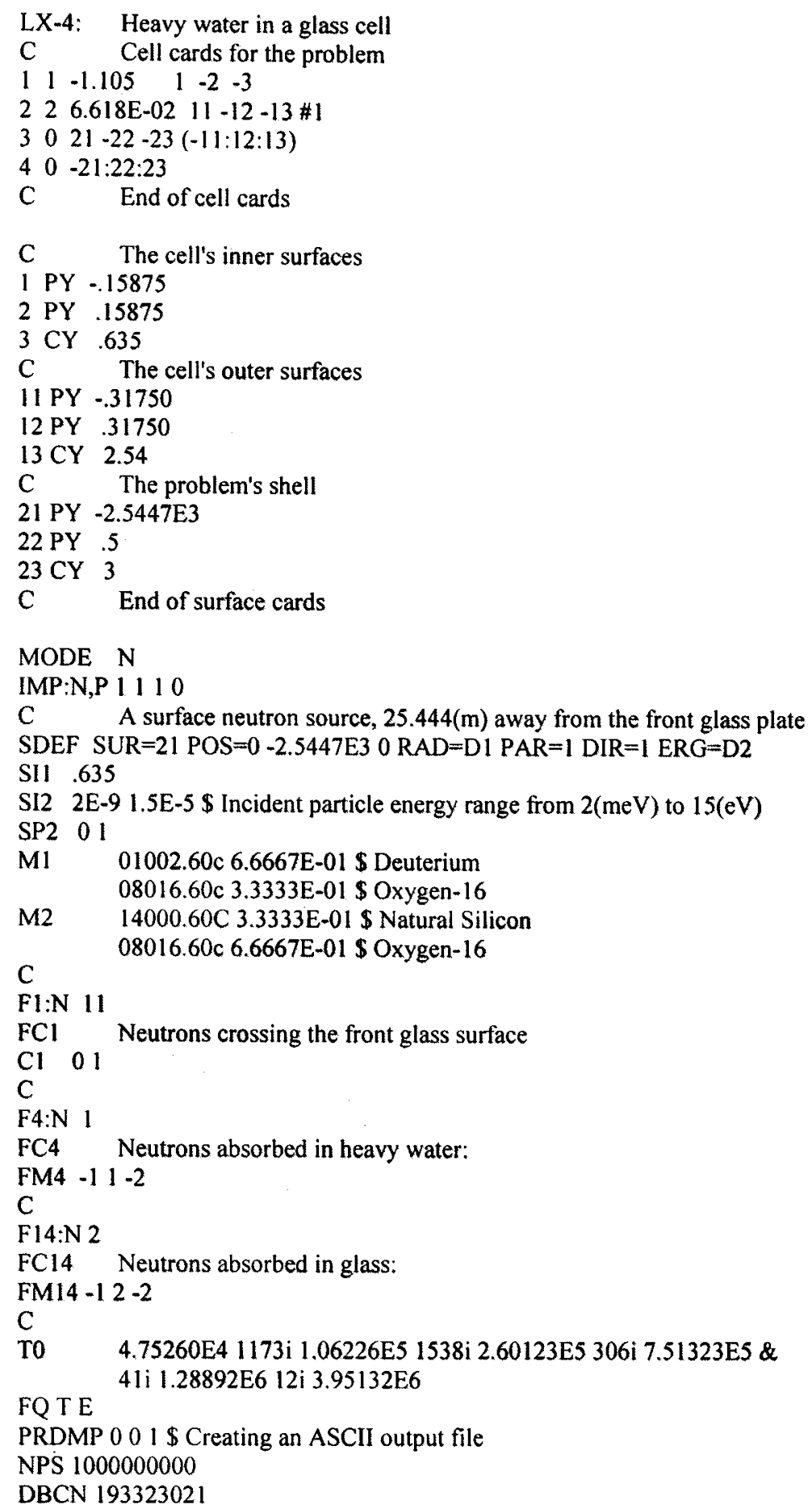

Figure A-5: MCNP deck (No Gd). 


\section{Appendix B - Details of Uncertainty Analysis}

\section{B.1 The Thermal Region}

Table B-1 gives a summary of results from the five uncertainty determination methods described in Section VII.A.

The final uncertainties, given on the fourth line of Table B-1 as well as in Table VI envelop the range of plausible values for resonance parameters resulting from these five methods.

The first uncertainty determination method was a variation on the combined transmission and capture fit described in Section V. It is called the two-step method and it is described in Section VII.A. The results of this approach are given on the fifth line of Table B-1.

The second method quantified uncertainties not associated with the liquid samples and their particular beam collimation system. The method is described in Section VII.A. The results of this method are given on lines 6-8 of Table B-1. A three-sample metal thermal transmission-only result is given on line 9 of Table B-1.

The third method for estimating uncertainties quantified uncertainties not associated with capture flux normalization. The method is described in Section VII.A The results are given on line 10 of Table B-1.

The fourth method estimated the uncertainty due to capture normalization. The method is described in Section VII.A. This sensitivity study fit capture data only. It did not vary capture normalization. Therefore the differences between these results and the final KAPL/RPI results, where capture normalization was varied and reached a value of 0.9 , is a measure of the sensitivity of results to the decision to normalize capture data to transmission results. The results of the capture-only fit are given on line 11 of Table B-1

The fifth method of uncertainty isolated and estimated the uncertainties associated with reproducing a given transmission result. The ${ }^{155} \mathrm{Gd}$-enriched liquid sample named LX-4 was measured in transmission twice within two weeks under similar experimental conditions. The differences in resonance parameters between single-sample SAMMY fits from these two experiments is shown on the last line of Table B-1. Emphasis should be placed on the results for the ${ }^{155} \mathrm{Gd}$ resonance since this is the dominant isotope in this enriched sample (see Table II). 
These are predominantly capture resonances, so both transmission and capture experiments essentially measure capture. The capture cross section is proportional to the neutron widths of the doublet. Radiation width is a more subtle parameter to extract from the data. Table B-1 shows that the resonance parameters calculated by the various methods are often outside the error bands around the KAPL/RPI central values. However, the central values are based on renormalizing capture during a simultaneous transmission-dominated combined fit.

Table B-1 Summary of Thermal Fitting Results. The Variability of the Results Indicates the Internal Consistency of the Data And Has Been Used to Estimate Uncertainties in Resonance Parameters

\begin{tabular}{|c|c|c|c|c|c|c|}
\hline & $\begin{array}{l}{ }^{155} \mathrm{Gd} \\
\mathrm{E}_{0}, \mathrm{eV}\end{array}$ & $\begin{array}{l}{ }^{155} \mathrm{Gd} \Gamma_{\gamma} \\
\mathrm{meV}\end{array}$ & $\begin{array}{l}{ }^{155} \mathrm{Gd} \Gamma_{\mathrm{n}} \\
\mathrm{meV}\end{array}$ & $\begin{array}{l}{ }^{157} \mathrm{Gd} \\
\mathrm{E}_{0}, \mathrm{eV}\end{array}$ & $\begin{array}{l}{ }^{157} \mathrm{Gd} \Gamma_{\gamma} \\
\mathrm{meV}\end{array}$ & $\begin{array}{l}{ }^{157} \mathrm{Gd} \Gamma_{\mathrm{n}} \\
\mathrm{meV}\end{array}$ \\
\hline ENDF & 0.0268 & 108.0 & 0.104 & 0.0314 & 106.0 & 0.4704 \\
\hline $\begin{array}{l}\text { Uncertainties- } \\
\text { from Moller }\end{array}$ & 0.0002 & 1 & 0.002 & 0.0002 & 1 & 0.0080 \\
\hline KAPL/RPI & 0.025 & 104.4 & 0.097 & 0.032 & 107.3 & 0.428 \\
\hline Est uncertainty & 0.003 & 2.5 & 0.003 & 0.003 & 3 & 0.004 \\
\hline $\begin{array}{l}\text { Two-step } \\
\text { method }\end{array}$ & 0.025 & 101.9 & 0.094 & 0.032 & 106.4 & 0.424 \\
\hline $\begin{array}{l}1 \text {-mil metal } \\
\text { only }\end{array}$ & $\begin{array}{l}\text { FIXED TO } \\
\text { ENDF }\end{array}$ & $\begin{array}{l}\text { FIXED TO } \\
\text { ENDF }\end{array}$ & $\begin{array}{l}\text { FIXED TO } \\
\text { ENDF }\end{array}$ & 0.029 & 104.7 & 0.442 \\
\hline $\begin{array}{l}2 \text {-mil metal } \\
\text { only }\end{array}$ & $\begin{array}{l}\text { FIXED TO } \\
\text { ENDF }\end{array}$ & $\begin{array}{l}\text { FIXED TO } \\
\text { ENDF }\end{array}$ & $\begin{array}{l}\text { FIXED TO } \\
\text { ENDF }\end{array}$ & 0.030 & 106.3 & 0.432 \\
\hline $\begin{array}{l}5 \text {-mil metal } \\
\text { only }\end{array}$ & $\begin{array}{l}\text { FIXED TO } \\
\text { ENDF }\end{array}$ & $\begin{array}{l}\text { FIXED TO } \\
\text { ENDF }\end{array}$ & $\begin{array}{l}\text { FIXED TO } \\
\text { ENDF }\end{array}$ & 0.030 & 107.1 & 0.428 \\
\hline Metals only & $\begin{array}{l}\text { FIXED TO } \\
\text { ENDF }\end{array}$ & $\begin{array}{l}\text { FIXED TO } \\
\text { ENDF }\end{array}$ & $\begin{array}{l}\text { FIXED TO } \\
\text { ENDF }\end{array}$ & 0.029 & 104.2 & 0.437 \\
\hline Trans only & 0.029 & 109.6 & 0.108 & 0.032 & 120.2 & 0.426 \\
\hline $\begin{array}{l}\text { Capture-only } \\
\text { not } \\
\text { renormalized }\end{array}$ & 0.027 & 107.6 & 0.119 & 0.031 & 105.5 & 0.468 \\
\hline $\begin{array}{l}\text { Delta LX-4 } \\
\text { transmission }\end{array}$ & 0.000 & 0.8 & 0.003 & 0.000 & 0.1 & 0.016 \\
\hline
\end{tabular}




\section{Appendix B - Details of Uncertainty Analysis}

\section{B.2 The Epithermal Region}

In addition to the SAMMY Bayesian errors, three methods of uncertainty analysis in the epithermal region are described in Section VII.B. The methods are capture-data only, SAMMY fits with unconstrained radiation widths, and SAMMY fits including transmission background variables $(A+B / \sqrt{E})$. The latter method was only employed in the 10-80 eV region. Table B-2 has a column for each of these uncertainties in each resonance parameter, labeled $\Delta \mathrm{cap}, \Delta \mathrm{con}$, and $\Delta \mathrm{bkg}$, respectively. Also the Bayesian SAMMY errors, column labeled $\triangle \mathrm{SAM}$ in Table B-2, propagated from the errors on each data point are also listed. These SAMMY errors are from the final combined fit to transmission and capture data. The largest uncertainty on each resonance parameter is used as the final uncertainty in Table VII in Section VI.B.2. When all estimates of uncertainty are zero in Table VII, the implication is that that variable was not included in the SAMMY fits. 
Table B-2 Summary of Epithermal Uncertainty Sensitivity Results

\begin{tabular}{|c|c|c|c|c|c|c|c|c|c|c|c|c|c|}
\hline $\begin{array}{r}E \\
e V\end{array}$ & $\begin{array}{c}\Delta \text { cap } \\
\text { eV }\end{array}$ & $\begin{array}{c}\Delta \mathrm{con} \\
\mathrm{eV}\end{array}$ & $\begin{array}{c}\triangle S A M \\
e V\end{array}$ & $\begin{array}{l}\Gamma Y \\
\text { meV }\end{array}$ & $\begin{array}{r}\Delta \mathrm{cap} \\
\mathrm{meV}\end{array}$ & $\begin{array}{r}\Delta \mathrm{con} \\
\mathrm{meV}\end{array}$ & $\begin{array}{r}\Delta \mathrm{bkg} \\
\mathrm{meV}\end{array}$ & $\begin{array}{r}\triangle \mathrm{SAM} \\
\mathrm{meV}\end{array}$ & $\begin{array}{l}\text { Tn } \\
\mathrm{meV}\end{array}$ & $\begin{array}{r}\Delta \text { cap } \\
\text { meV }\end{array}$ & $\begin{array}{r}\Delta \mathrm{con} \\
\text { meV }\end{array}$ & $\begin{array}{r}\Delta \mathrm{bkg} \\
\mathrm{meV}\end{array}$ & $\begin{array}{r}\triangle S A M \\
\text { meV }\end{array}$ \\
\hline $.012 E+0$ & $E+0$ & $E+0$ & $2 E-4$ & 128.0 & 1.2 & 0.0 & NA & & & 0.01 & .00 & NA & \\
\hline $.573 E+0$ & $3 E-4$ & $O E+O$ & $2 E-4$ & 107.1 & 0.2 & 0.0 & NA & 0.4 & 1.71 & 0.00 & .00 & NA & \\
\hline $2.829 E+0$ & $2 E-4$ & $O E+O$ & $3 E-4$ & 109.7 & 0.2 & 0.8 & NA & 0.9 & 0.38 & 0.00 & .00 & $\mathrm{NA}$ & \\
\hline $3.616 \mathrm{E}+0$ & $2 E-4$ & $2 E-4$ & $3 E-3$ & 130.0 & 0.0 & 0.0 & NA & 0.0 & 0.05 & 0.02 & 0.01 & NA & .00 \\
\hline $6.306 \mathrm{E}+0$ & $1 E-4$ & $O E+O$ & $2 E-4$ & 108.8 & 0.1 & 0.1 & NA & 0.6 & 2.20 & 0.00 & 0.00 & NA & .01 \\
\hline $18 E+0$ & $E+0$ & $O E+O$ & $4 E-4$ & 109.5 & 0.0 & 0.0 & NA & 1.2 & 1.16 & 0.00 & .00 & $\mathrm{NA}$ & 0.01 \\
\hline $91 E+0$ & $3 E-3$ & $O E+0$ & $5 E-4$ & 106.0 & 24.0 & 0.0 & NA & 1.1 & 0.20 & 0.04 & 0.00 & $\mathrm{NA}$ & 0.00 \\
\hline $51 E+1$ & $1 E-3$ & $1 E-3$ & $9 E-4$ & 118.7 & 1.7 & 43.4 & 0.9 & 0.8 & 0.78 & 0.08 & 0.03 & .01 & 0.01 \\
\hline $57 E+1$ & $E-3$ & $5 E-2$ & $4 E-3$ & 88.3 & 0.4 & 82.6 & 0.7 & 1.0 & 0.19 & 0.17 & .02 & .02 & 0.02 \\
\hline 1. $196 \mathrm{E}+1$ & $8 E-3$ & $0 E+0$ & $3 E-4$ & 127.2 & 22.6 & 4.9 & 4.0 & 0.7 & 1.12 & 0.03 & 0.02 & .04 & 0.00 \\
\hline $1.235 \mathrm{E}+1$ & $0 \mathrm{E}+0$ & $O E+0$ & $O E+0$ & 58.6 & 0.0 & 0.0 & 0.0 & 0.0 & 4.65 & 0.00 & 0.00 & .00 & 0.00 \\
\hline $48 E+1$ & $9 E-3$ & $O E+0$ & $2 E-4$ & 125.0 & 13.6 & 0.1 & 2.6 & 0.4 & 3.43 & 0.02 & .00 & .09 & 0.01 \\
\hline 1. $620 \mathrm{E}+1$ & $5 E-3$ & $2 E-3$ & $1 E-3$ & 133.7 & 32.6 & 5.0 & 16.2 & 3.7 & 0.44 & 0.03 & 0.01 & .03 & 0.01 \\
\hline $1.678 \mathrm{E}+1$ & $1 E-2$ & $1 E-3$ & $1 E-4$ & 111.8 & 7.4 & 1.3 & 0.5 & 0.3 & 13.85 & 0.52 & .02 & .30 & 0.01 \\
\hline $1.773 \mathrm{E}+1$ & $E-3$ & $2 E-3$ & $8 E-4$ & 127.6 & 3.3 & 45.0 & 1.2 & 0.9 & 0.47 & 0.03 & 0.04 & .01 & 0.00 \\
\hline $36 E+1$ & $E-2$ & $O E+0$ & $3 E-4$ & 118.4 & 5.7 & 0.7 & 1.8 & 0.7 & 4.54 & 0.06 & 0. & 0.11 & 0.01 \\
\hline $1 \mathrm{E}+1$ & $E-2$ & $\mathrm{OE}+0$ & $2 E-4$ & 105.7 & 7.8 & 0.1 & 0.8 & 0.5 & & 0.45 & 0.01 & .30 & 0.02 \\
\hline $2.097 \mathrm{E}+1$ & $2 E-2$ & $O E+0$ & $3 E-4$ & 135.1 & 20.8 & 1.0 & 1.6 & 0.9 & 1.59 & 0.53 & 0.01 & .40 & 0.03 \\
\hline $9 E+1$ & $2 E-2$ & $\mathrm{OE}+0$ & $2 E-3$ & 75.6 & 32.0 & 3.3 & 8.6 & 5.9 & 0 & 0.04 & 01 & 0.08 & 0.01 \\
\hline $2.230 \mathrm{E}+1$ & $4 E-2$ & $1 E-3$ & $4 E-4$ & 95.6 & 36.6 & 0.3 & 5.7 & 0.5 & & 0.79 & 0.01 & .62 & 0.03 \\
\hline $2.251 \mathrm{E}+1$ & $2 \mathrm{E}-1$ & $O E+0$ & $2 E-3$ & 74.9 & 137.9 & 0.0 & 13.9 & 2.4 & 16.57 & 11.46 & 0.10 & .07 & 0.29 \\
\hline $2.328 E+1$ & $3 E-2$ & $\mathrm{OE}+0$ & $2 E-3$ & 136.4 & 34.8 & 0.4 & 24.6 & 5.7 & 1.33 & 0.27 & 0.00 & 0.13 & 0.03 \\
\hline $2.360 E+1$ & $2 E-2$ & $O E+O$ & $8 E-4$ & 143.1 & 14.5 & 0.4 & 4.3 & 1.6 & 2.91 & 0.07 & 0.00 & .08 & 0.02 \\
\hline $2.535 E+1$ & $1 E-2$ & $O E+0$ & $5 E-4$ & 132.7 & 25.2 & 0.2 & 4.9 & 1.0 & 1.99 & 0.02 & 0.00 & .06 & 0.01 \\
\hline $2.751 \mathrm{E}+1$ & $2 E-3$ & $\mathrm{OE}+0$ & $1 E-3$ & 140.1 & 17.5 & 1.1 & 16.8 & 1.9 & 1.31 & 0.04 & 0.00 & 0.06 & 0.01 \\
\hline 2. $950 \mathrm{E}+1$ & $2 E-2$ & $\mathrm{OE}+0$ & $5 E-4$ & 112.6 & 1.8 & 0.6 & 1.4 & 1.0 & 4.80 & 0.09 & 0.01 & 0.11 & 0.01 \\
\hline $3.005 E+1$ & $2 E-2$ & $0 \mathrm{E}+0$ & $3 E-4$ & 125.2 & 11.2 & 0.1 & 2.0 & 0.7 & 11.11 & 0.48 & 0.00 & 0.23 & 0.02 \\
\hline $3.166 \mathrm{E}+1$ & $1 E-2$ & $\mathrm{OE}+0$ & $9 E-4$ & 135.6 & 15.7 & 1.5 & 5.7 & 2.2 & 1.24 & 0.07 & 0.00 & 0.04 & 0.01 \\
\hline $3.307 \mathrm{E}+1$ & $1 E-3$ & $2 E-1$ & $O E+0$ & 112.0 & 0.8 & 34.6 & 0.0 & 0.0 & 1.61 & 0.00 & 0.64 & 0.00 & 0.00 \\
\hline $3.314 \mathrm{E}+1$ & $3 E-2$ & $7 E-3$ & $2 E-4$ & 97.9 & 0.7 & 2.8 & 0.8 & 0.3 & 14.21 & 0.38 & 1.55 & 0.44 & 0.02 \\
\hline 3. $343 \mathrm{E}+1$ & $O E+0$ & $3 E-1$ & $0 E+0$ & 115.0 & 3.6 & 87.4 & 0.0 & 0.0 & 0.93 & 0.00 & 3.55 & 0.00 & 0.00 \\
\hline $3.473 E+1$ & $2 E-2$ & $1 E-3$ & $6 E-4$ & 130.8 & 3.3 & 3.6 & 0.0 & 1.5 & 6.83 & 0.00 & 0.05 & 0.15 & 0.02 \\
\hline $3.539 \mathrm{E}+1$ & $1 E-2$ & $1 E-3$ & $9 E-4$ & 138.5 & 14.0 & 0.8 & 3.2 & 2.4 & 2.17 & 0.02 & 0.00 & 0.06 & 0.01 \\
\hline
\end{tabular}


Table B-2 (continued) Summary of Epithermal Uncertainty Sensitivity Results

\begin{tabular}{|c|c|c|c|c|c|c|c|c|c|c|c|c|c|}
\hline $\begin{array}{r}E \\
\mathrm{eV}\end{array}$ & $\begin{array}{c}\Delta \text { cap } \\
\mathrm{eV}\end{array}$ & $\begin{array}{c}\Delta c o n \\
e V\end{array}$ & $\begin{array}{c}\triangle S A M \\
\mathrm{eV}\end{array}$ & $\begin{array}{l}\Gamma \gamma \\
\text { meV }\end{array}$ & $\begin{array}{r}\Delta \mathrm{cap} \\
\text { meV }\end{array}$ & $\begin{array}{r}\Delta \mathrm{con} \\
\text { meV }\end{array}$ & $\begin{array}{r}\Delta \mathrm{bkg} \\
\text { meV }\end{array}$ & $\begin{array}{r}\triangle S A M \\
\text { meV }\end{array}$ & $\begin{array}{l}\text { In } \\
\text { meV }\end{array}$ & $\begin{array}{r}\Delta \text { cap } \\
\text { meV }\end{array}$ & $\begin{array}{r}\Delta \mathrm{con} \\
\mathrm{meV}\end{array}$ & $\begin{array}{r}\Delta \mathrm{bkg} \\
\mathrm{meV}\end{array}$ & $\begin{array}{r}\triangle S A M \\
\text { meV }\end{array}$ \\
\hline $3.686 \mathrm{E}+1$ & $\mathrm{OE}+0$ & $0 E+0$ & $O E+0$ & 56.0 & 0.0 & 0.0 & 0.0 & 0.0 & 34.00 & 0.00 & 0.00 & 0.00 & .00 \\
\hline $3.707 E+1$ & $3 E-3$ & $0 E+0$ & $6 E-4$ & 138.5 & 5.5 & 6.4 & 5.0 & 1.1 & 8.29 & 0.32 & 0.07 & 0.28 & 0.02 \\
\hline 3. $893 \mathrm{E}+1$ & $1 E-2$ & $1 \mathrm{E}-2$ & $1 E-3$ & 125.2 & 8.2 & 55.1 & 1.9 & 1.0 & 1.25 & 0.03 & 0.07 & 0.04 & 0.01 \\
\hline $3.930 \mathrm{E}+1$ & $\mathrm{OE}+0$ & $O E+0$ & $O E+0$ & 56.0 & 0.0 & 0.0 & 0.0 & 0.0 & 39.00 & 0.00 & 0.00 & 0.00 & 0.00 \\
\hline $4.008 \mathrm{E}+1$ & $1 E-2$ & $1 E-3$ & $1 E-3$ & 119.4 & 5.1 & 43.4 & 2.6 & 1.0 & 1.61 & 0.16 & 0.01 & 0.07 & 0.01 \\
\hline $4.273 E+1$ & $O E+O$ & $O E+O$ & $0 E+0$ & 56.0 & 0.0 & 0.0 & 0.0 & 0.0 & 3.06 & 0.00 & 0.00 & 0.00 & 0.00 \\
\hline 4. $383 E+1$ & $6 E-2$ & $7 E-2$ & $1 E-3$ & 138.7 & 57.3 & 93.4 & 0.2 & 0.2 & 18.33 & 3.11 & 9.00 & 0.76 & 0.13 \\
\hline $4.411 \mathrm{E}+1$ & $3 E-2$ & $4 \mathrm{E}-2$ & $1 E-3$ & 118.9 & 23.4 & 74.9 & 2.9 & 0.9 & 9.45 & 1.18 & 4.81 & 0.05 & 0.08 \\
\hline $4.598 \mathrm{E}+1$ & $2 E-2$ & $7 E-3$ & $1 E-3$ & 128.0 & 5.7 & 4.7 & 0.3 & 1.0 & 2.26 & 0.13 & 0.02 & 0.03 & 0.01 \\
\hline $4.679 E+1$ & $2 E-2$ & $6 E-3$ & $1 E-3$ & 140.5 & 31.1 & 22.1 & 5.4 & 2.6 & 10.16 & 0.07 & 0.42 & 0.32 & 0.07 \\
\hline $4.718 E+1$ & $4 E-2$ & $2 E-2$ & $9 E-3$ & 89.1 & 2.3 & 5.5 & 3.5 & 8.5 & 2.38 & 0.61 & 0.51 & 0.11 & 0.12 \\
\hline $4.763 E+1$ & $6 E-3$ & $3 E-3$ & $6 E-3$ & 106.7 & 6.0 & 4.3 & 2.9 & 9.7 & 0.39 & 0.02 & 0.03 & 0.00 & 0.01 \\
\hline $4.868 E+1$ & $3 E-2$ & $1 E-3$ & $3 E-4$ & 118.1 & 8.7 & 3.6 & 1.8 & 0.6 & 26.75 & 0.33 & 0.09 & 0.54 & 0.04 \\
\hline $4.963 E+1$ & $7 E-2$ & $1 E-2$ & $3 E-3$ & 89.4 & 1.4 & 39.9 & 0.1 & 1.0 & 3.10 & 1.08 & 0.17 & 0.04 & 0.04 \\
\hline $5.125 E+1$ & $3 E-2$ & $5 E-3$ & $6 E-4$ & 131.0 & 11.6 & 34.3 & 0.7 & 0.8 & 20.26 & 0.06 & 0.59 & 0.48 & 0.06 \\
\hline $5.201 \mathrm{E}+1$ & $3 E-2$ & $4 E-3$ & $6 \mathrm{E}-4$ & 137.4 & 20.7 & 22.0 & 1.0 & 1.7 & 20.91 & 0.51 & 0.75 & 0.46 & 0.06 \\
\hline $.289 E+1$ & $2 E-2$ & $5 E-3$ & $2 E-3$ & 83.0 & 33.1 & 21.2 & 3.3 & 6.7 & 1.21 & 0.17 & 0.09 & 0.00 & 0.02 \\
\hline $5.362 E+1$ & $2 E-2$ & $\mathrm{OE}+0$ & $7 E-4$ & 142.7 & 34.3 & 5.2 & 3.8 & 1.6 & 8.65 & 0.19 & 0.05 & 0.23 & 0.03 \\
\hline $5.612 \mathrm{E}+1$ & $1 E-2$ & $6 E-3$ & $1 E-3$ & 124.6 & 3.8 & 40.6 & 1.2 & 0.9 & 2.55 & 0.11 & 0.06 & 0.07 & 0.01 \\
\hline $5.826 \mathrm{E}+1$ & $3 E-2$ & $1 E-3$ & $4 E-4$ & 138.1 & 15.4 & 7.0 & 3.5 & 0.7 & 32.02 & 0.22 & 0.23 & 0.62 & 0.05 \\
\hline $5.931 \mathrm{E}+1$ & $1 E-2$ & $3 E-3$ & $9 E-4$ & 135.2 & 9.8 & 38.1 & 0.4 & 0.9 & 6.87 & 0.41 & 0.20 & 0.13 & 0.03 \\
\hline $273 E+1$ & $2 E-2$ & $O E+O$ & $8 E-4$ & 148.6 & 29.7 & 3.8 & 7.0 & 1.5 & 8.45 & 0.45 & 0.03 & 0.21 & 0.03 \\
\hline $6.403 \mathrm{E}+1$ & $2 E-3$ & $1 E-3$ & $6 E-3$ & 112.4 & 0.1 & 42.4 & 0.2 & 1.0 & 0.49 & 0.05 & 0.03 & 0.04 & 0.01 \\
\hline $6.521 E+1$ & $1 E-2$ & $1 E-3$ & $9 E-4$ & 96.6 & 22.4 & 1.3 & 2.3 & 1.5 & 31.67 & 4.93 & 0.06 & 0.71 & 0.13 \\
\hline $6.640 \mathrm{E}+1$ & $5 E-1$ & $2 E-3$ & $2 E-2$ & 120.8 & 3.0 & 3.9 & 6.1 & 11.4 & 0.54 & 0.12 & 0.02 & 0.39 & 0.04 \\
\hline $6.653 \mathrm{E}+1$ & $1 E-2$ & $1 E-3$ & $1 E-3$ & 131.0 & 55.5 & 13.1 & 1.9 & 1.8 & 15.65 & 1.84 & 0.17 & 0.19 & 0.07 \\
\hline $6.944 E+1$ & $1 \mathrm{E}-1$ & $4 E-3$ & $1 E-3$ & 141.5 & 123.8 & 116.8 & 4.2 & 1.0 & 12.13 & 3.75 & 0.14 & 0.39 & 0.05 \\
\hline $7.434 \mathrm{E}+1$ & $\mathrm{OE}+0$ & $O E+O$ & $O E+O$ & 50.4 & 0.0 & 0.0 & 0.0 & 0.0 & 60.00 & 0.00 & 0.00 & 0.00 & 0.00 \\
\hline $7.600 \mathrm{E}+1$ & $3 E-2$ & $9 E-3$ & $5 E-3$ & 88.9 & 1.1 & 45.9 & 0.6 & 1.0 & 2.04 & 0.91 & 0.06 & 0.15 & 0.03 \\
\hline $7.685 E+1$ & $1 E-2$ & $5 E-3$ & $2 E-3$ & 113.7 & 0.3 & 64.7 & 0.8 & 1.0 & 2.99 & 0.27 & 0.14 & 0.12 & 0.03 \\
\hline $7.763 \mathrm{E}+1$ & $3 E-3$ & $1 E-2$ & $4 E-3$ & 112.6 & 0.7 & 21.1 & 0.4 & 1.0 & 0.88 & 0.15 & 0.03 & 0.06 & 0.01 \\
\hline $7.875 E+1$ & $2 E-2$ & $6 E-2$ & $2 E-3$ & 113.2 & 5.2 & 31.9 & 0.0 & 4.0 & 8.32 & 0.49 & 1.15 & 0.00 & 0.06 \\
\hline 8.004E+1 & $7 E-2$ & $4 \mathrm{E}-2$ & $4 E-4$ & 80.1 & 6.5 & 3.6 & NA & 0.5 & 79.72 & 21.28 & 0.24 & NA & 0.13 \\
\hline
\end{tabular}


Table B-2 (continued) Summary of Epithermal Uncertainty Sensitivity Results

\begin{tabular}{|c|c|c|c|c|c|c|c|c|c|c|c|}
\hline $\begin{array}{r}E \\
\text { eV }\end{array}$ & $\begin{array}{c}\Delta c a p \\
e V\end{array}$ & $\begin{array}{c}\Delta c o n \\
e V\end{array}$ & $\begin{array}{c}\triangle S A M \\
\mathrm{eV}\end{array}$ & $\begin{array}{l}\text { Ir } \\
\text { meV }\end{array}$ & $\begin{array}{r}\Delta c a p \\
\text { meV }\end{array}$ & $\begin{array}{r}\Delta \mathrm{con} \\
\mathrm{meV}\end{array}$ & $\begin{array}{r}\triangle S A M \\
\text { meV }\end{array}$ & $\begin{array}{l}\Gamma n \\
\text { meV }\end{array}$ & $\begin{array}{r}\Delta \mathrm{cap} \\
\text { meV }\end{array}$ & $\begin{array}{r}\Delta \mathrm{con} \\
\text { meV }\end{array}$ & $\begin{array}{r}\triangle \mathrm{SAM} \\
\text { meV }\end{array}$ \\
\hline $8.005 E+1$ & $6 E-2$ & $1 \mathrm{E}+0$ & $O E+0$ & 112.0 & 4.0 & 1.2 & 0.0 & 0.31 & 3.39 & 0.56 & 0.00 \\
\hline $8.090 \mathrm{E}+1$ & $3 E-1$ & $2 E-1$ & $\mathrm{OE}+0$ & 112.0 & 1.0 & 26.6 & 0.0 & 1.44 & 0.02 & 0.09 & 0.00 \\
\hline $8.130 E+1$ & $4 E-2$ & $4 E-2$ & $1 E-3$ & 111.5 & 3.5 & 39.0 & 1.0 & 24.31 & 2.01 & 0.56 & 0.12 \\
\hline $8.210 \mathrm{E}+1$ & $3 E-2$ & $4 E-2$ & $2 E-3$ & 102.3 & 1.7 & 71.3 & 1.0 & 7.07 & 0.60 & 0.07 & 0.04 \\
\hline $8.397 E+1$ & $2 E-2$ & $2 E-2$ & $2 E-3$ & 115.2 & 2.2 & 40.7 & 1.0 & 10.31 & 0.01 & 0.14 & 0.06 \\
\hline 8. $491 \mathrm{E}+1$ & $1 E-3$ & $1 E-2$ & $3 E-3$ & 113.1 & 26.9 & 40.5 & 1.0 & 2.22 & 0.30 & 0.01 & 0.02 \\
\hline $8.555 E+1$ & $O E+0$ & $\mathrm{OE}+0$ & $O E+O$ & 58.6 & 0.0 & 0.0 & 0.0 & 5.11 & 0.00 & 0.00 & 0.00 \\
\hline $8.717 \mathrm{E}+1$ & $2 \mathrm{E}-2$ & $3 E-2$ & $1 E-3$ & 141.9 & 11.9 & 6.7 & 1.9 & 11.09 & 0.39 & 0.16 & 0.05 \\
\hline $9.051 \mathrm{E}+1$ & $1 E-2$ & $2 E-2$ & $3 E-3$ & 113.3 & 35.7 & 86.0 & 1.0 & 2.51 & 0.22 & 0.13 & 0.03 \\
\hline $9.240 \mathrm{E}+1$ & $0 E+0$ & $O E+O$ & $O E+0$ & 58.6 & 0.0 & 0.0 & 0.0 & 142.00 & 0.00 & 0.00 & 0.00 \\
\hline $9.247 \mathrm{E}+1$ & $6 E-3$ & $2 E-2$ & $7 E-3$ & 112.6 & 4.4 & 19.5 & 1.0 & 2.14 & 0.05 & 0.00 & 0.06 \\
\hline $9.290 \mathrm{E}+1$ & $5 E-3$ & $3 E-2$ & $4 E-3$ & 113.2 & 31.8 & 45.6 & 1.0 & 3.48 & 0.07 & 0.05 & 0.06 \\
\hline $9.399 E+1$ & $1 E-2$ & $4 E-3$ & $6 E-3$ & 112.6 & 16.4 & 40.1 & 1.0 & 0.64 & 0.09 & 0.02 & 0.01 \\
\hline $9.571 E+1$ & $3 E-2$ & $3 E-2$ & $2 E-3$ & 113.3 & 18.7 & 52.7 & 1.0 & 7.07 & 0.16 & 0.36 & 0.06 \\
\hline $9.640 \mathrm{E}+1$ & $2 E-1$ & $2 E-1$ & $O E+0$ & 112.0 & 7.0 & 50.0 & 0.0 & 3.76 & 0.70 & 0.02 & 0.00 \\
\hline $9.656 \mathrm{E}+1$ & $E-2$ & $1 E-1$ & $2 E-3$ & 103.3 & 21.7 & 36.9 & 1.0 & 22.02 & 0.42 & 0.12 & 0.13 \\
\hline $9.830 \mathrm{E}+1$ & $2 E-2$ & $3 E-2$ & $1 E-3$ & 147.1 & 22.1 & 10.4 & 3.0 & 11.74 & 0.44 & 0.22 & 0.06 \\
\hline $9.987 \mathrm{E}+1$ & $7 E-3$ & $1 E-1$ & $2 E-2$ & 111.3 & 5.7 & 12.3 & 10.8 & 2.52 & 0.16 & 0.20 & 0.21 \\
\hline $1.002 \mathrm{E}+2$ & $3 E-2$ & $6 E-2$ & $3 E-3$ & 100.3 & 4.5 & 28.2 & 3.2 & 43.20 & 0.10 & 1.27 & 0.44 \\
\hline $1.007 E+2$ & $2 E-2$ & $8 E-2$ & $8 E-3$ & 88.8 & 3.3 & 39.0 & 5.7 & 47.69 & 6.99 & 2.71 & 1.10 \\
\hline $1.012 E+2$ & $3 E-2$ & $9 E-2$ & $2 E-2$ & 122.6 & 14.6 & 1.4 & 11.1 & 1.32 & 0.01 & 0.21 & 0.08 \\
\hline $1.014 \mathrm{E}+2$ & $E-2$ & $1 E-2$ & $2 E-2$ & 144.5 & 25.5 & 8.5 & 12.4 & 2.07 & 0.15 & 0.15 & 0.19 \\
\hline 1. $.020 E+2$ & $3 E-2$ & $2 E-2$ & $7 E-3$ & 112.5 & 13.5 & 53.8 & 1.0 & 1.52 & 0.03 & 0.06 & 0.04 \\
\hline $.044 E+2$ & $E-2$ & $9 E-2$ & $5 E-3$ & 113.1 & 2.1 & 77.9 & 1.0 & 5.19 & 1.37 & 1.21 & 0.11 \\
\hline $1.049 \mathrm{E}+2$ & $1 E-2$ & $8 E-2$ & $2 E-3$ & 102.7 & 1.7 & 0.8 & 1.5 & 66.18 & 36.82 & 1.46 & 0.33 \\
\hline $.058 E+2$ & $E-1$ & $1 E-1$ & $1 E-2$ & 138.3 & 23.3 & 10.4 & 11.9 & 5.73 & 1.34 & 0.64 & 0.26 \\
\hline $1.061 \mathrm{E}+2$ & $6 E-2$ & $8 E-2$ & $1 E-2$ & 106.9 & 15.6 & 9.0 & 9.1 & 10.70 & 1.04 & 1.92 & 0.68 \\
\hline $.071 E+2$ & $E-2$ & $0 E+0$ & $4 E-3$ & 113.2 & 34.8 & 82.1 & 1.0 & 8.81 & 0.88 & 1.64 & 0.20 \\
\hline $1.075 \mathrm{E}+2$ & $2 E-2$ & $6 E-2$ & $1 E-2$ & 123.9 & 29.4 & 13.9 & 8.6 & 3.51 & 0.66 & 1.27 & 0.19 \\
\hline $.094 E+2$ & $\mathbf{E}+0$ & $2 E-2$ & $3 E-3$ & 114.6 & 1.6 & 0.4 & 1.0 & 7.28 & 0.54 & 0.00 & 0.08 \\
\hline 1. $105 E+2$ & $O E+0$ & $7 E-2$ & $1 E-3$ & 138.2 & 50.3 & 9.3 & 1.5 & 48.47 & 21.33 & 0.34 & 0.16 \\
\hline $1.124 \mathrm{E}+2$ & $E-2$ & $4 E-2$ & $2 E-3$ & 86.5 & 26.5 & 65.6 & 1.0 & 9.08 & 0.07 & 0.22 & 0.06 \\
\hline 1. $138 \mathrm{E}+2$ & $3 E-2$ & $5 E-2$ & $1 E-3$ & 129.4 & 2.4 & 19.7 & 3.1 & 20.29 & 1.39 & 0.29 & 0.10 \\
\hline $1.154 \mathrm{E}+2$ & $3 E-2$ & $6 E-2$ & $1 E-3$ & 141.7 & 17.7 & 18.2 & 3.4 & 22.24 & 0.94 & 0.75 & 0.11 \\
\hline 1. $166 \mathrm{E}+2$ & $1 E-2$ & $6 E-2$ & $2 E-3$ & 115.0 & 29.0 & 83.0 & 1.0 & 20.86 & 1.34 & 0.41 & 0.14 \\
\hline 1. $187 E+2$ & $1 E-2$ & $2 E-2$ & $4 E-3$ & 112.5 & 10.5 & 47.1 & 1.0 & 2.51 & 0.36 & 0.09 & 0.03 \\
\hline $1.208 E+2$ & $1 E-2$ & $O E+0$ & $9 E-4$ & 126.5 & 33.6 & 5.4 & 0.9 & 139.18 & 39.78 & 0.92 & 0.34 \\
\hline $1.233 E+2$ & $5 E-2$ & $4 E-2$ & $2 E-3$ & 160.0 & 127.9 & 146.7 & 0.5 & 40.25 & 5.13 & 6.26 & 0.31 \\
\hline 1. $242 E+2$ & $8 E-2$ & $2 E-2$ & $4 E-3$ & 112.5 & 43.1 & 49.1 & 3.3 & 150.64 & 22.32 & 23.15 & 1.61 \\
\hline 1. $245 E+2$ & $3 E-2$ & $O E+0$ & $1 E-2$ & 120.9 & 12.6 & 21.3 & 10.9 & 4.28 & 0.19 & 1.33 & 0.25 \\
\hline 1. $261 \mathrm{E}+2$ & $2 E-2$ & $O E+O$ & $2 E-3$ & 113.7 & 26.6 & 64.6 & 1.0 & 14.61 & 0.37 & 0.11 & 0.10 \\
\hline $1.285 \mathrm{E}+2$ & $2 E-2$ & $1 E-2$ & $8 E-3$ & 110.2 & 15.9 & 29.6 & 1.0 & 1.65 & 0.16 & 0.04 & 0.04 \\
\hline $1.298 \mathrm{E}+2$ & $O E+O$ & $1 E-2$ & $5 E-3$ & 110.2 & 20.2 & 35.8 & 1.0 & 3.39 & 0.11 & 0.26 & 0.06 \\
\hline $1.308 E+2$ & $1 E-2$ & $1 E-2$ & $4 E-3$ & 149.4 & 1.6 & 32.9 & 7.2 & 21.70 & 2.65 & 0.71 & 0.31 \\
\hline 1. $314 E+2$ & $O E+0$ & $O E+0$ & $9 E-3$ & 129.7 & 5.4 & 1.2 & 9.7 & 11.46 & 0.73 & 0.74 & 0.43 \\
\hline $1.330 \mathrm{E}+2$ & $O E+0$ & $1 E-2$ & $5 E-3$ & 138.9 & 18.0 & 11.6 & 1.0 .2 & 5.35 & 0.43 & 0.16 & 0.09 \\
\hline $1.339 E+2$ & $1 E-2$ & $1 E-2$ & $5 E-3$ & 110.3 & 23.2 & 27.5 & 1.0 & 3.40 & 0.18 & 0.00 & 0.05 \\
\hline $1.351 E+2$ & $2 E-2$ & $2 E-2$ & $6 E-3$ & 110.8 & 60.3 & 62.0 & 1.0 & 1.89 & 0.10 & 0.04 & 0.03 \\
\hline $1.380 E+2$ & $1 E-2$ & $8 E-2$ & $3 E-3$ & 121.3 & 12.9 & 75.3 & 1.0 & 85.51 & 32.07 & 14.69 & 1.08 \\
\hline 1. $382 \mathrm{E}+2$ & $1 E-1$ & $2 E-1$ & $2 E-2$ & 101.1 & 2.9 & 11.8 & 1.0 & 21.44 & 7.14 & 9.08 & 0.97 \\
\hline $1.389 E+2$ & $2 E-1$ & $1 E-1$ & $2 E-2$ & 93.7 & 5.0 & 2.1 & 7.8 & 37.03 & 0.66 & 11.40 & 1.59 \\
\hline $1.394 E+2$ & $3 E-2$ & $5 E-2$ & $4 E-3$ & 96.6 & 42.0 & 68.1 & 1.0 & 42.94 & 11.20 & 8.19 & 0.70 \\
\hline
\end{tabular}


Table B-2 (continued) Summary of Epithermal Uncertainty Sensitivity Results

\begin{tabular}{|c|c|c|c|c|c|c|c|c|c|c|c|}
\hline $\begin{array}{r}E \\
e V\end{array}$ & $\begin{array}{c}\Delta \text { cap } \\
\text { eV }\end{array}$ & $\begin{array}{c}\Delta \mathrm{con} \\
\mathrm{eV}\end{array}$ & $\begin{array}{c}\triangle S A M \\
\mathrm{eV}\end{array}$ & $\begin{array}{l}\Gamma Y \\
\text { meV }\end{array}$ & $\begin{array}{r}\Delta c a p \\
\text { meV }\end{array}$ & $\begin{array}{r}\Delta \mathrm{Con} \\
\text { meV }\end{array}$ & $\begin{array}{r}\triangle S A M \\
\text { meV }\end{array}$ & $\begin{array}{l}\text { In } \\
\text { mev }\end{array}$ & $\begin{array}{r}\Delta \text { cap } \\
\text { mev }\end{array}$ & $\begin{array}{r}\Delta \mathrm{con} \\
\mathrm{meV}\end{array}$ & A \\
\hline $.400 \mathrm{E}+2$ & $\mathbf{E}+0$ & $O E+0$ & $0 \mathrm{E}+0$ & 58.6 & 0.0 & 0.0 & 0.0 & 78.80 & 0.00 & 0.00 & \\
\hline $.406 \mathrm{E}+2$ & $\mathbf{E}-2$ & $2 E-2$ & $9 E-3$ & 131.2 & 6.8 & 2.3 & 11.3 & 4.89 & 0.34 & 0.23 & \\
\hline $1.413 E+2$ & $\mathbf{E}-2$ & $1 E-2$ & $1 E-2$ & 18.1 & 2.3 & 0.4 & 10.8 & 1.69 & 0.08 & 0.05 & \\
\hline $1.438 \mathrm{E}+2$ & $E-2$ & $O E+0$ & $1 E-3$ & 28.3 & 29.6 & 1.3 & 1.6 & 60.63 & 13.69 & 0.03 & 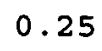 \\
\hline $1.457 \mathrm{E}+2$ & $O E+0$ & $O E+0$ & $4 E-3$ & 46.8 & 16.9 & 4.0 & 9.0 & 6.52 & 0.27 & 0.06 & 0.0 \\
\hline $1.470 \mathrm{E}+2$ & $O E+0$ & $0 \mathrm{E}+0$ & $5 E-3$ & 31.5 & 1.2 & 3.7 & 10.4 & 5.26 & 0.20 & 0.06 & \\
\hline $1.484 \mathrm{E}+2$ & $3 E-1$ & $1 E-2$ & $4 E-2$ & 14.1 & 1.3 & 2.7 & 11.1 & 8.56 & 0.86 & 0.23 & 0.6 \\
\hline $1.482 \mathrm{E}+2$ & $2 E-1$ & $1 E-2$ & $1 E-2$ & 18.0 & 22.4 & 4.9 & 8.6 & 45.69 & 9.66 & 1.99 & $? 8$ \\
\hline $1.486 \mathrm{E}+2$ & $5 E-2$ & $2 \mathrm{E}-2$ & $1 E-2$ & 135.3 & 27.1 & 13.2 & 9.9 & 24.21 & 1.49 & 0.26 & 1.1 \\
\hline $1.495 \mathrm{E}+2$ & $E-2$ & $1 E-2$ & $6 E-3$ & 10.7 & 5.9 & 41.5 & 1.0 & 35.74 & 2.48 & 0.30 & \\
\hline$E+2$ & $E-2$ & $3 E-2$ & $6 E-3$ & 111.8 & 15.7 & 37.5 & 1.0 & 77.51 & 25.91 & 12.09 & .7 \\
\hline 1.506 & $E-2$ & $O E+0$ & $5 E-3$ & 76.1 & 11.9 & 26.9 & 4.3 & 23.35 & 6.99 & 4.79 & 0.7 \\
\hline .523 & $E-2$ & $1 E-2$ & $5 E-3$ & 147.6 & 38.0 & 31.7 & 10.2 & 6.22 & 0.77 & 0.47 & .0 \\
\hline .538 & $E-2$ & $E-2$ & $1 E-2$ & 56.2 & 30.5 & 17.1 & 12.6 & 1.07 & 0.24 & 0.10 & \\
\hline .56 & $E-1$ & $7 E-2$ & $6 \mathrm{E}-3$ & 11.7 & 57.7 & 76.5 & 1.0 & 28.65 & 11.67 & 7.65 & 0.9 \\
\hline .567 & $E-2$ & $2 E-2$ & $8 E-3$ & 142.4 & 45.4 & 44.9 & 7.3 & 12.63 & 4.69 & 4.35 & 0.5 \\
\hline $3+2$ & $E+0$ & $0 \mathrm{E}+0$ & $O E+0$ & 58.6 & 0.0 & 0.0 & 0.0 & 2.83 & 0.00 & 0.00 & 0.0 \\
\hline 1.600 & $E-2$ & $0 \mathrm{E}+0$ & $3 E-3$ & 12.7 & 2.1 & 47.7 & 1.0 & 10.31 & 0.48 & 0.00 & 0.1 \\
\hline+2 & E-2 & $O E+O$ & $2 E-3$ & 151.8 & 17.6 & 2.2 & 4.9 & 21.63 & 0.79 & .00 & 0.1 \\
\hline$\Xi+2$ & $E-1$ & $O E+0$ & $6 E-3$ & 98.4 & 7.1 & 0.0 & 2.9 & 157.92 & 1.43 & 0.00 & 2.3 \\
\hline$\Xi+2$ & -2 & $O E+0$ & $8 E-3$ & 99.7 & 16.1 & 81.5 & 1.0 & 22.85 & 6.23 & .00 & 0.8 \\
\hline+2 & $E-2$ & $O E+0$ & $4 E-3$ & 122.6 & 1.0 & 0.0 & 5.6 & 30.58 & 3.65 & 0.00 & 0.3 \\
\hline+2 & $\Xi+0$ & $O E+0$ & $4 E-2$ & 100.0 & 0.0 & 0.0 & 0.0 & 3.33 & 0.00 & 0.00 & 0.0 \\
\hline+2 & $E-1$ & $O E+0$ & $2 E-2$ & 93.2 & 4.4 & 0.0 & 10.1 & 3.42 & 0.03 & 0.00 & 0.1 \\
\hline+2 & $a-1$ & $O E+0$ & $1 E-2$ & 82.4 & 30.9 & 0.0 & 7.8 & 8.32 & 1.29 & 0.00 & 0.2 \\
\hline+2 & $E-1$ & $O E+0$ & $9 E-2$ & 85.5 & 2.7 & & 8.7 & 4.85 & 0.12 & 0.00 & 0.4 \\
\hline+2 & $a-1$ & $\mathrm{OE}+0$ & $6 E-3$ & 103.5 & 14.5 & 0.0 & 3.3 & 116.73 & 42.13 & & 2.02 \\
\hline$E+2$ & $E-1$ & $O E+0$ & $8 E-3$ & 113.1 & 8.6 & 61.9 & 1.0 & 18.32 & 0.97 & 0.00 & 1.0 \\
\hline $5 E+2$ & $E-1$ & $0 E+0$ & $3 E-3$ & 113.6 & 31.0 & 76.4 & 1.0 & 33.16 & 1.82 & 0.00 & 23 \\
\hline .738 & $E+0$ & $E+0$ & $O E+0$ & 30.1 & 0.0 & 0. & 0.0 & 86.00 & 0.00 & 0.00 & 0.01 \\
\hline+2 & $g-2$ & $O E+O$ & $4 E-3$ & 112.3 & 4.3 & 44.7 & 0.5 & 4.25 & 0.56 & & \\
\hline+2 & $E-2$ & $O E+0$ & $1 E-2$ & 132.5 & 10.8 & 0 & 1.0 & 13.39 & 2.15 & 00 & 0.0 \\
\hline+2 & -2 & $E+0$ & $5 E-3$ & 143.5 & 0.5 & 16.3 & 1.0 & 16.97 & 96 & & .2 \\
\hline $.803 E+2$ & $E-2$ & $E+0$ & $1 E-2$ & 112.2 & 27.4 & 37.8 & 10.7 & 9.69 & 0.04 & 00 & 0.3 \\
\hline+2 & -2 & $E+0$ & $1 E-2$ & 112.2 & 32.5 & 41.8 & 1.0 & 11.51 & 1.59 & & $0.3 c$ \\
\hline $.839 E+2$ & $E-2$ & $O E+0$ & $6 E-3$ & 101.1 & 19.0 & 89.9 & 1.0 & 34.36 & 7.62 & 00 & .47 \\
\hline+2 & -2 & $E+0$ & $1 E-2$ & 112.4 & 8.4 & 58.3 & 1.0 & 3.45 & 0.70 & & 0.07 \\
\hline $.857 E+2$ & $E+0$ & $O E+0$ & $O E+0$ & 52.5 & 0.0 & 0.0 & 0.0 & 4.00 & 00 & & 0.0 \\
\hline+2 & $E-2$ & $\mathbf{E}+0$ & $3 E-3$ & 115.4 & 20.0 & 3.6 & 1.0 & 31.11 & 1.48 & & 0.28 \\
\hline+2 & $E-2$ & $E+0$ & $8 E-3$ & 98.7 & 5.8 & 8 & 1.0 & .88 & 4.99 & & .2 \\
\hline+2 & $E-I$ & $E+0$ & $3 E-3$ & 102.2 & 12.5 & 89 & 1.0 & 64.56 & 4.64 & & 0.47 \\
\hline+2 & $E-1$ & $O E+0$ & $2 E-3$ & 113.5 & 16.2 & 46.5 & 1.0 & 64.37 & 7.87 & & .37 \\
\hline+2 & $E-1$ & $\mathrm{OE}+\mathrm{O}$ & $1 E-3$ & 92.0 & 4.5 & 0 . & 0.8 & 200.19 & 96.41 & & 0.53 \\
\hline$\Xi+2$ & $E-1$ & $\mathrm{OE}+0$ & $E-2$ & 60.5 & 43.2 & 0 . & 5.1 & 77.10 & 51.94 & & 1.33 \\
\hline+2 & $1 E-2$ & $\mathrm{OE}+0$ & $E-3$ & 158.9 & 37.8 & 0 & 2.9 & 54.68 & 11.21 & 0 . & 0.25 \\
\hline$E+2$ & $0 E+0$ & $O E+0$ & $O E+0$ & 58.8 & 0.0 & 0 . & 0.0 & 97.00 & 0.00 & & 0.00 \\
\hline $.034 E+2$ & $0 E+0$ & $O E+0$ & $2 E-2$ & 129.3 & 10.2 & 0 . & 1.1 & 8.35 & & & 0.27 \\
\hline+2 & $4 E-2$ & $O E+0$ & $2 E-2$ & 106.8 & 3.8 & 0.0 & 10.2 & 2.01 & 0.11 & 00 & 0.07 \\
\hline $2.078 \mathrm{E}+2$ & $4 E-2$ & $O E+0$ & $3 E-3$ & 146.6 & 18.9 & 0.0 & 2.8 & 106.00 & 30. & & 0.6 \\
\hline $2.091 \mathrm{E}+2$ & $2 E-1$ & $O E+O$ & $2 E-2$ & 115.8 & 7.7 & 0.0 & 10.6 & 5.76 & 1.16 & 0.00 & 0.1 \\
\hline & $1 E-2$ & $O E+0$ & $8 E-3$ & 137.7 & 20.8 & 0.0 & 10.9 & 14.18 & & & 0.2 \\
\hline $.116 \mathrm{E}+2$ & $2 E-2$ & $O E+0$ & $2 E-2$ & 98.7 & 6.7 & 0.0 & 7.6 & 44.66 & 0.14 & 0.00 & 1.3 \\
\hline
\end{tabular}


Table B-2 (continued) Summary of Epithermal Uncertainty Sensitivity Results

\begin{tabular}{|c|c|c|c|c|c|c|c|c|c|c|c|}
\hline $\begin{array}{r}E \\
\mathrm{eV}\end{array}$ & $\begin{array}{c}\Delta \text { cap } \\
e V\end{array}$ & $\begin{array}{c}\Delta \mathrm{con} \\
\mathrm{eV}\end{array}$ & $\begin{array}{c}\triangle \mathrm{SAM} \\
\mathrm{eV}\end{array}$ & $\begin{array}{l}\Gamma Y \\
\text { meV }\end{array}$ & $\begin{array}{r}\Delta c a p \\
\text { meV }\end{array}$ & $\begin{array}{r}\Delta \mathrm{con} \\
\mathrm{meV}\end{array}$ & $\begin{array}{r}\triangle S A M \\
\text { meV }\end{array}$ & $\begin{array}{l}\Gamma n \\
\text { meV }\end{array}$ & $\begin{array}{r}\Delta \text { cap } \\
\text { meV }\end{array}$ & $\begin{array}{r}\Delta \mathrm{con} \\
\mathrm{meV}\end{array}$ & $\begin{array}{r}\triangle S A M \\
\text { meV }\end{array}$ \\
\hline $2.123 E+2$ & $2 E-2$ & $0 E+0$ & $2 E-2$ & 104.7 & 0.7 & 0.0 & 10.1 & 3.12 & 0.03 & 0.00 & 0.12 \\
\hline $2.137 E+2$ & $1 E-2$ & $\mathrm{OE}+0$ & $2 E-2$ & 102.4 & 0.5 & 0.0 & 9.9 & 9.19 & 0.18 & 0.00 & 0.28 \\
\hline $2.148 E+2$ & $O E+0$ & $O E+O$ & $6 E-3$ & 130.6 & 16.0 & 0.0 & 6.1 & 46.58 & 3.97 & 0.00 & 0.54 \\
\hline $2.172 \mathrm{E}+2$ & $O E+0$ & $0 E+0$ & $7 E-3$ & 120.8 & 7.2 & 0.0 & 9.2 & 19.85 & 0.92 & 0.00 & 0.28 \\
\hline $2.186 \mathrm{E}+2$ & $2 E-2$ & $\mathrm{OE}+0$ & $8 E-3$ & 140.1 & 10.9 & 0.0 & 10.8 & 9.61 & 0.11 & 0.00 & 0.15 \\
\hline $2.202 \mathrm{E}+2$ & $8 E-2$ & $O E+O$ & $1 E-2$ & 148.9 & 18.8 & 0.0 & 11.8 & 8.26 & 0.41 & 0.00 & 0.16 \\
\hline $2.222 \mathrm{E}+2$ & $3 E-2$ & $O E+0$ & $3 E-3$ & 80.4 & 22.4 & 0.0 & 1.8 & 54.87 & 24.78 & 0.00 & 0.31 \\
\hline $2.233 E+2$ & $O E+0$ & $O E+0$ & $\mathrm{OE}+0$ & 64.2 & 0.0 & 0.0 & 0.0 & 301.00 & 0.00 & 0.00 & 0.00 \\
\hline $2.249 \mathrm{E}+2$ & $1 E-2$ & $2 E-2$ & $6 E-3$ & 95.1 & 27.0 & 117.8 & 1.0 & 111.48 & 56.25 & 8.47 & 1.30 \\
\hline $2.279 E+2$ & $1 E-2$ & $2 \mathrm{E}-2$ & $4 E-3$ & 104.5 & 77.5 & 103.0 & 1.0 & 52.42 & 2.03 & 3.21 & 0.44 \\
\hline $2.295 E+2$ & $1 E-2$ & $2 E-2$ & $5 E-3$ & 99.1 & 55.4 & 65.1 & 0.5 & 49.55 & 2.39 & 2.67 & 0.51 \\
\hline $2.309 E+2$ & $5 E-2$ & $O E+0$ & $8 E-3$ & 122.1 & 121.9 & 124.3 & 1.0 & 33.00 & 0.12 & 1.97 & 0.51 \\
\hline $2.314 \mathrm{E}+2$ & $\mathrm{OE}+\mathrm{O}$ & $0 \mathrm{E}+0$ & $O E+0$ & 62.0 & 0.0 & 0.0 & 0.0 & 46.00 & 0.00 & 0.00 & 0.00 \\
\hline $2.329 E+2$ & $\mathrm{OE}+0$ & $O E+O$ & $8 E-3$ & 99.7 & 74.0 & 90.9 & 1.0 & 18.88 & 0.63 & 1.62 & 0.28 \\
\hline $2.359 \mathrm{E}+2$ & $2 E-1$ & $1 E-2$ & $8 E-3$ & 74.4 & 22.6 & 62.6 & 1.0 & 68.82 & 9.20 & 10.97 & 1.19 \\
\hline $2.373 E+2$ & $1 E-1$ & $4 E-2$ & $6 E-3$ & 102.1 & 100.7 & 119.0 & 1.0 & 29.78 & 0.77 & 0.97 & 0.33 \\
\hline $2.380 \mathrm{E}+2$ & $O E+0$ & $O E+0$ & $O E+0$ & 100.0 & 0.0 & 0.0 & 0.0 & 223.60 & 0.00 & 0.00 & 0.00 \\
\hline $2.396 E+2$ & $3 E-2$ & $\mathrm{OE}+0$ & $2 E-3$ & 124.8 & 17.5 & 0.0 & 1.4 & 247.68 & 39.00 & 0.00 & 0.90 \\
\hline $2.432 \mathrm{E}+2$ & $\mathrm{OE}+0$ & $O E+0$ & $3 E-3$ & 89.1 & 21.2 & 0.0 & 2.3 & 46.76 & 16.29 & 0.00 & 0.30 \\
\hline $2.452 \mathrm{E}+2$ & $2 E-2$ & $O E+0$ & $1 E-2$ & 97.8 & 5.8 & 0.0 & 9.0 & 3.25 & 0.05 & 0.00 & 0.06 \\
\hline $2.468 \mathrm{E}+2$ & $O E+O$ & $O E+O$ & $7 E-3$ & 117.9 & 7.4 & 0.0 & 9.2 & 19.81 & 0.48 & 0.00 & 0.30 \\
\hline $2.488 E+2$ & $1 E-2$ & $0 \mathrm{E}+0$ & $1 E-2$ & 115.7 & 5.7 & 0.0 & 10.3 & 5.01 & 0.02 & 0.00 & 0.10 \\
\hline $2.505 \mathrm{E}+2$ & $2 E-2$ & $O E+0$ & $1 E-2$ & 126.2 & 10.1 & 0.0 & 120.8 & 8.18 & 0.14 & 0.00 & 0.16 \\
\hline $2.524 \mathrm{E}+2$ & $\mathrm{OE}+0$ & $0 E+0$ & $O E+0$ & 52.4 & 0.0 & 0.0 & 0.0 & 127.00 & 0.00 & 0.00 & 0.00 \\
\hline $2.532 \mathrm{E}+2$ & $3 E-2$ & $O E+0$ & $1 E-2$ & 101.2 & 0.8 & 0.0 & 8.6 & 26.16 & 1.15 & 0.00 & 0.74 \\
\hline $2.549 E+2$ & $O E+0$ & $0 E+0$ & $7 E-3$ & 129.8 & 1.7 & 0.0 & 9.8 & 18.58 & 0.53 & 0.00 & 0.29 \\
\hline $2.565 \mathrm{E}+2$ & $4 E-2$ & $O E+O$ & $6 \mathrm{E}-2$ & 100.5 & 0.3 & .0 & 1.0 .0 & 1.46 & 0.01 & 0.00 & 0.09 \\
\hline $2.580 E+2$ & $1 E-2$ & $O E+0$ & $1 E-2$ & 90.8 & 1.0 & 0.0 & 6.5 & 39.69 & 0.23 & 0.00 & 1.05 \\
\hline $2.592 \mathrm{E}+2$ & $1 E-2$ & $0 E+0$ & $2 E-2$ & 101.8 & 0.8 & 0.0 & 1.0 .0 & 5.68 & 0.06 & 0.00 & 0.15 \\
\hline $2.605 \mathrm{E}+2$ & $0 \mathrm{E}+0$ & $0 E+0$ & $8 E-3$ & 124.4 & 12.2 & 0.0 & 8.3 & 30.53 & 2.53 & 0.00 & 0.47 \\
\hline $2.626 \mathrm{E}+2$ & $1 E-2$ & $O E+O$ & $1 \mathrm{E}-2$ & 103.8 & 0.7 & 0.0 & 9.8 & 5.18 & 0.01 & 0.00 & 0.10 \\
\hline $2.649 \mathrm{E}+2$ & $O E+O$ & $\mathrm{OE}+0$ & $1 E-2$ & 107.0 & 3.5 & 0.0 & 1.0 .0 & 6.61 & 0.10 & 0.00 & 0.15 \\
\hline $.660 \mathrm{E}+2$ & $O E+0$ & $0 E+0$ & $1 E-2$ & 109.6 & 3.8 & 0.0 & 1.0 .1 & 7.86 & 0.23 & 0.00 & 0.17 \\
\hline $2.685 E+2$ & $0 \mathrm{E}+0$ & $O E+0$ & $1 E-2$ & 135.4 & 20.6 & 0.0 & 1.0 .0 & 17.05 & 0.92 & 0.00 & 0.33 \\
\hline $2.696 E+2$ & $3 E-2$ & $\mathrm{OE}+0$ & $1 E-2$ & 124.6 & 21.5 & 0.0 & 8.9 & 53.62 & 12.71 & 0.00 & 1.65 \\
\hline $2.724 \mathrm{E}+2$ & $2 E-2$ & $2 E-2$ & $1 E-2$ & 100.8 & 56.8 & 46.0 & 1.0 & 11.64 & 0.57 & 1.03 & 0.20 \\
\hline $2.774 \mathrm{E}+2$ & $6 E-2$ & $2 E-2$ & $4 E-3$ & 118.7 & 14.3 & 268.7 & 1.0 & 36.48 & 55.52 & 5.38 & 0.27 \\
\hline $2.794 \mathrm{E}+2$ & $2 E-2$ & $O E+0$ & $3 E-2$ & 98.5 & 1.8 & 0.0 & 9.9 & 2.24 & 0.15 & 0.00 & 0.10 \\
\hline $2.820 \mathrm{E}+2$ & $O E+O$ & $\mathrm{OE}+0$ & $O E+0$ & 49.1 & 0.0 & 0.0 & 0.0 & 145.00 & 0.00 & 0.00 & 0.00 \\
\hline $2.823 E+2$ & $5 E-2$ & $4 E-2$ & $5 E-3$ & 109.5 & 31.0 & 95.2 & 1.0 & 74.95 & 28.67 & 4.82 & 0.70 \\
\hline $2.842 \mathrm{E}+2$ & $1 E-1$ & $1 E-1$ & $2 E-2$ & 100.6 & 7.4 & 27.0 & 1.0 & 18.84 & 1.85 & 0.04 & 0.60 \\
\hline $2.852 \mathrm{E}+2$ & $5 E-2$ & $3 E-2$ & $2 E-2$ & 147.4 & 41.9 & 27.3 & 11.5 & 14.38 & 1.80 & 2.16 & 0.40 \\
\hline $2.879 E+2$ & $3 E-2$ & $4 E-2$ & $1 E-2$ & 101.1 & 14.8 & 46.9 & 1.0 & 25.11 & 3.36 & 0.53 & 0.53 \\
\hline $2.890 \mathrm{E}+2$ & $3 E-2$ & $2 \mathrm{E}-2$ & $2 E-2$ & 135.1 & 31.1 & 17.3 & 1.1 .2 & 20.79 & 0.95 & 2.33 & 0.71 \\
\hline $2.911 \mathrm{E}+2$ & $2 E-2$ & $3 E-2$ & $1 E-2$ & 101.7 & 10.0 & 52.5 & 1.0 & 50.53 & 9.45 & 0.39 & 0.92 \\
\hline $2.924 \mathrm{E}+2$ & $7 E-2$ & $5 E-2$ & $2 E-2$ & 128.7 & 28.4 & 10.8 & 1.1 .0 & 19.59 & 1.38 & 1.54 & 0.59 \\
\hline $.930 E+2$ & $O E+0$ & $\mathrm{OE}+0$ & $O E+0$ & 71.0 & 0.0 & 0.0 & 0.0 & 352.00 & 0.00 & 0.00 & 0.00 \\
\hline $2.942 \mathrm{E}+2$ & $1 E-2$ & $\mathrm{OE}+0$ & $6 E-3$ & 126.2 & 30.8 & 6.7 & 5.8 & 48.61 & 7.55 & 0.09 & 0.59 \\
\hline $2.958 E+2$ & $8 E-2$ & $O E+0$ & $2 E-2$ & 100.0 & 7.1 & 0.7 & 9.9 & 2.92 & 0.85 & 0.02 & 0.12 \\
\hline $2.980 E+2$ & $8 E-2$ & $1 E-1$ & $1 E-2$ & 109.8 & 11.7 & 10.8 & 1.0 .3 & 3.40 & 0.71 & 0.08 & 0.25 \\
\hline
\end{tabular}

